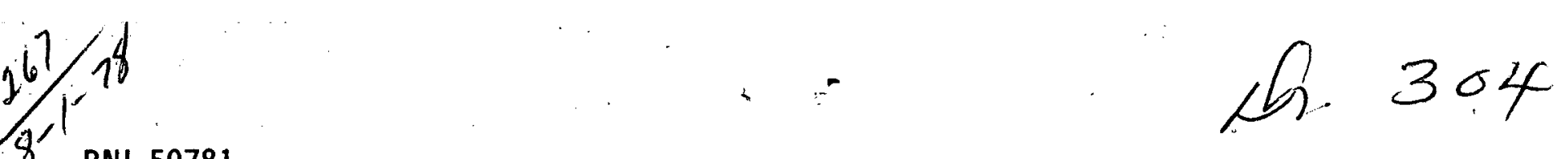

\title{
RADIATION EFFECTS ON ION EXCHANGE MATERIALS
}

\author{
A Report on Radiation Effects Program \\ for
}

Oak Ridge National Laboratory

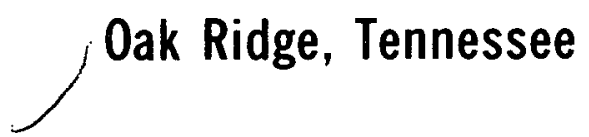

T.E. Gangwer, M. Goldstein, and K.K.S. Pillay
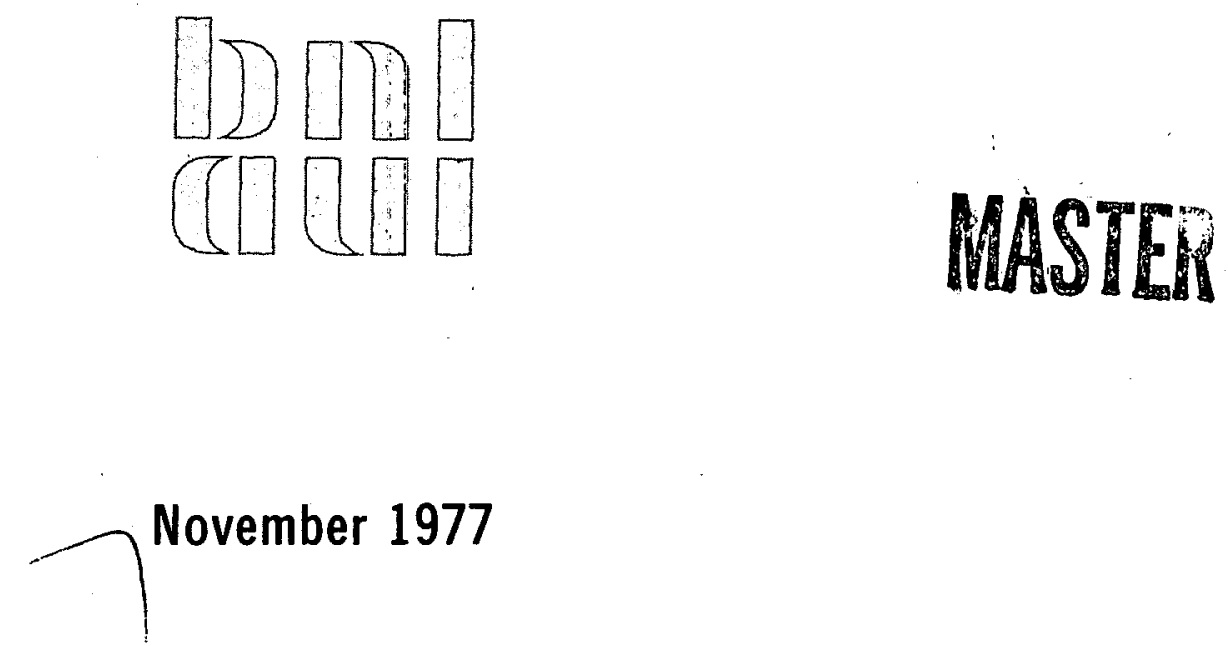

DEPARTMENT OF ENERGY AND ENVIRONMENT

BROOKHAVEN NATIONAL LABORATORY ASSOCIATED UNIVERSITIES, INC. UNDER CONTRACT NO. EY-76-C-02-0016 WITH THE

UNITED STATES DEPARTMENT OF ENERGY 


\section{DISCLAIMER}

This report was prepared as an account of work sponsored by an agency of the United States Government. Neither the United States Government nor any agency Thereof, nor any of their employees, makes any warranty, express or implied, or assumes any legal liability or responsibility for the accuracy, completeness, or usefulness of any information, apparatus, product, or process disclosed, or represents that its use would not infringe privately owned rights. Reference herein to any specific commercial product, process, or service by trade name, trademark, manufacturer, or otherwise does not necessarily constitute or imply its endorsement, recommendation, or favoring by the United States Government or any agency thereof. The views and opinions of authors expressed herein do not necessarily state or reflect those of the United States Government or any agency thereof. 


\section{DISCLAIMER}

Portions of this document may be illegible in electronic image products. Images are produced from the best available original document. 
BNL 50781

UC-70

(Nuclear Waste Management - TID-4500)

\title{
RADIATION EFFECTS ON ION EXCHANGE MATERIALS
}

\author{
A Report on Radiation Effects Program \\ for \\ Oak Ridge National Laboratory \\ Oak Ridge, Tennessee
}

T.E. Gangwer, M. Goldstein*, and K.K.S. Pillay**

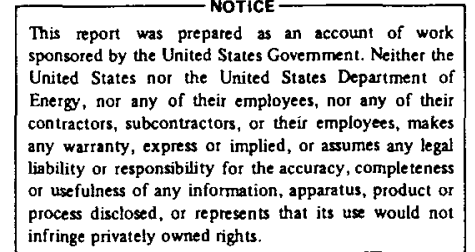

"Present address: Institute for Environment and Policy, East-West Center, 1717 East-West Rd., Honolulu, Hawaii 96822

**Associate Professor of Nuclear Engineering, The Pennsylvania State University, University Park, PA 16802

This document is

PUBLICLY RELEASABLE

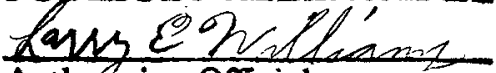

Authofizing Official

Date: $06 / 25 / 2007$

Research sponsored by the Division of Waste Handling, Waste Management, Production and Reprocessing, U.S. Department of Energy, Washington, D.C.

DEPARTMENT OF ENERGY AND ENVIRONMENT

BROOKHAVEN NATIONAL LABORATORY

UPTON, NEW YORK 11973 


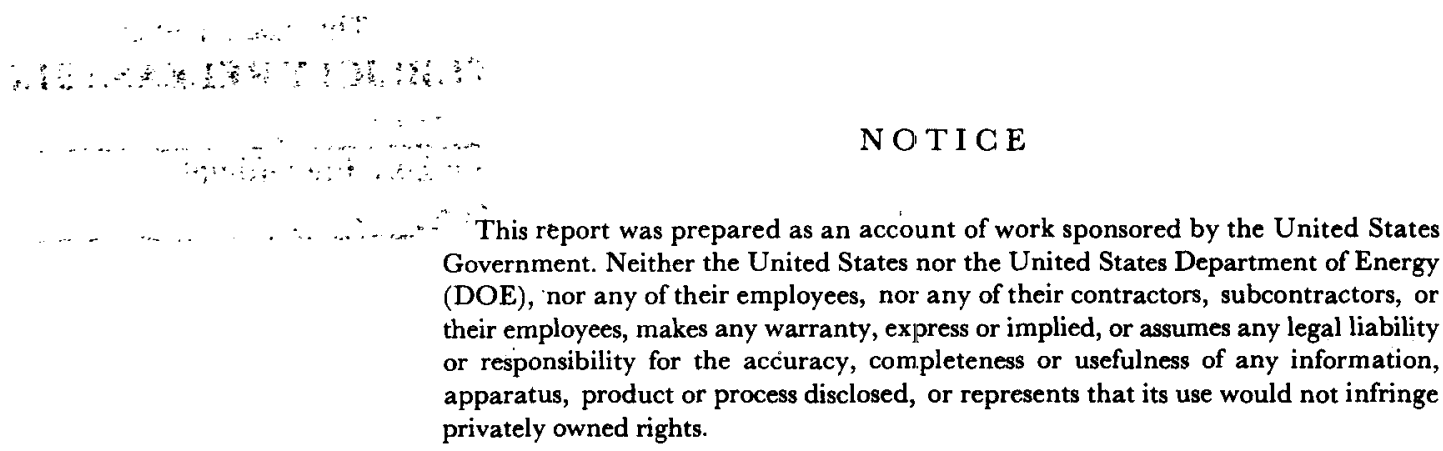
Government. Neither the United States nor the United States Department of Energy (DOE), nor any of their employees, nor any of their contractors, subcontractors, or their employees, makes any warranty, express or implied, or assumes any legal liability or responsibility for the accuracy, completeness or usefulness of any information, apparatus, product or process disclosed, or represents that its use would not infringe privately owned rights.

Printed in the United States of America Available from

National Technical Information Service

U.S. Department of Commerce

5285 Port Royal Road Springfield, VA 22161

Price: Printed Copy $\$ 8.00$; Microfiche $\$ 3.00$. 
TABLE OF CONTENTS

$\underline{\text { Page }}$

Forward . . . . . . . . . . . . . . . . . . . . . . . v

Abstract . . . . . . . . . . . . . . . . . . . . vi

I. Introduction . . . . . . . . . . . . . . . . . . . . 1

II. General Characteristics of Ion Exchange Materials . . . . . . . 5

II.1 Organic and Inorganic Exchangers . . . . . . . . . 5

II.2 Classification of Ion Exchangers . . . . . . . . . . 7

II.2.a Classification based on exchangeable species . . . . . . 7

II.2.b Classification based on functional groups . . . . . . . 7

II.2.c Classification based on preparative processes . . . . . 9

II.2.d Classification based on physical state . . . . . . . . 9

II.2.e Classification based on granulation . . . . . . . . 9

II.2.f Classification based on the rigldity of porous structure - 10

II.3 Properties of Ion Exchangers ............. 10

II.3.a Exchange capacity .................. 11

II.3.b Degree of cross-1inking . . . . . . . . . . . . 12

II.3.C Swelling and Moisture Content . . . . . . . . . . 13

II.4 Ion Exchange Process . . . . . . . . . . . . . . 14

II.5 Chemical Composition of Synthetic Organic Ion Exchangers . 15

IJ.. Experimental Data on the Effect of Radiation on Synthetic Ion

Exchangers ................... . . 24

III.I Resin (Trade name) ............... 25

III.2 Ionic Form ................. 26

III.3 Exchange Capacity . . . . . . . . . . . . 27

III.4 Radiation Source (Dose rate: $\operatorname{Rad} / \mathrm{Hr}$ ) . . . . . . . 28

III.5 Irracilation Condition(s) . . . . . . . . . . 28 
TABLE OF CONTENTS (Cont'd)

Page

III.6 Integral Dose (Rads) . . . . . . . . . . . . 28

III.7 Loss of Exchange Capacity (\%) . . . . . . . . . . . 29

III. 8 G-Value . . . . . . . . . . . . . . . 29

III.9 Other Parameters Measured . . . . . . . . . . 30

III.10 Reference Number and Notes . . . . . . . . . . . 31

IV. Generalizations on the Effects of Radiation on Ion Exchange 58

V. Radiation Effects on Ion Exchange Resins . . . . . . . . . 65

V.1 Polymerization-Type Cation-Exchange Resins . . . . . . . 65

V. 2 Other Types of Cation Exchange Resins . . . . . . . . 77

V. 3 Radiation-Protective-Effect of Some Cation Exchangers . . 78

V.4 Anion Exchange Resins . . . . . . . . . . . . 79

V.5 High Radiation Resistance of Some Anion Exchange Resins . . 88

V.6 Gas Evolution During the Radiolysis of Ion Exchange
Resins . . . . . . . . . . . . . 89

V.7 Mechanistic Considerations in the Radiolysis of Ion
Exchange Resins . . . . . . . . . . . 96

VI. Conclusions . . . . . . . . . . . . . . . . . . 105

VII. References . . . . . . . . . . . . . . . . . 108

Appendix I . . . . . . . . . . . . . . . . . . . . 117

Appendix II . . . . . . . . . . . . . . . . . . 128 
FORWARD

This study is part of the Actinide Partitioning and Transmutation Program which is a multisite effort coordinated at the Oak Ridge National Laboratory. The overall program objective is to evaluate the feasibility and incentives for partitioning the long-lived biologically significant isotopes found in fuel wastes and transmuting them to shorter-1ived or stable isotopes in power reactors. The BNL contribution to this program is the examination of the formation of radiation products in waste streams encountered in fuel reprocessing and refabrication plants which operate with a high degree of stream recycle.

We would like to acknowledge the valuable services of Pennsylvania State University, the University of Notre Dame Radiation Laboratory and Data Center, and the various U.S. ion exchange resin manufacturers for supplying information and materials necessary to prepare this report.

We are thankful to the ORNL and ERDA for their support of this literature study. Our special thanks to the IAEA, Vienna, and KETER Publishing House Ltd., Jerusalem for allowing us the use of the materials from their publications as appendices to this report. One of us (KKSP) wishes to express his gratitude to Brookhaven National Laboratory for the opportunity to partictpate in this study. 
An extensive literature review and data compilation has been completed on the radiation-damage of ion exchange resins. The primary goal of the study has been to review the available literature on ion exchange materials used in, as well as those with potential for use in, the nuclear fuel and waste reprocessing areas. The physical and chemical properties of ion exchangers are reviewed. Experimental parameters useful in characterizing the effects of radiation on synthetic ion exchange resins are identified or defined. In compiling the diverse types of data, an effort was made to present the experimental data or experimentally based parameters in a format that would be useful for inter-comparfing radiation effects on resins. When subject to radiation there are various general trends or qualitative effects displayed by the different types of resins. These radiation-trends and effects have been formulated into qualitative statements. The present day level of understanding of the behavior of resins under ionizing radiation is too limited to justify quantitative predictive modeling. The limitations and difficiencies of the literature are discussed and the experimentation needed to achieve quantitative modeling are outlined. 


\section{INTRODUCTION}

During the past century, a very large number of natural and synthetic materials have been identified as having ion exchange properties and today their applications in science and industry are rather extensive. Ever since the first synthesis of organic ion exchangers forty years ago, hundreds of different types of ion exchange materials, both organic and inorganic have been synthesized. From this bewildering assortment of synthetic ion exchangers, only a few types have emerged as materials of commercial value. Of these, a selected few have found vital applications in the nuclear process industry. The last three decades saw rapid growth in the applications of ion exchange materials in several areas of nuclear power generation, radioisotope processing, nuclear fuel reprocessing and radioactive waste treatment. Although the number of ion exchangers identified as potentially useful in nuclear process industry include many inorganic materials, the vast majority of the ion exchangers used today are the synthetic organic resins. One of the serious limitations of these ion exchange materials is their radiation chemical decomposition as a consequence of their exposure to ionizing radiations. Since inorganic ion exchangers show considerable superiority in this one respect, there have been renewed interest in developing inorganic ion exchangers to replace the functions of synthetic organic resins. While this effort is still maturing, synthetic organic ion exchangers dominate the process related applications in the nuclear industry.

This literature survey was undertaken to review the present knowledge on the radiation effects. on Ion exchange materials. It is part of an overall effort to examine the present knowledge of the effects of intense ionizing radiation on the degradation of process materlals, with the objective of predicting potential problems in the process streams. This survey is limited to the 
radiation induced chemical changes and the resultant changes in the properties of ion exchange materials. No detalled discussion of ion exchange technólogy and its applications are considered appropriate here. The basic principles of processing aqueous radioactive wastes and spent fuel materials using ion exchangers are reviewed in two recent monographs [77,78]. Brief reviews of the chemical nature and properties of ion exchange materials are included here to. orient the reader to the terminology used in this report and to show the relevance of estimating radiation effects on ion exchangers through a study of measurable properties that are of significance to process applications.

This literature survey has attempted to identify all published materials on the radiation effects on ion exchange materials. We have used the computerized literature search facilities of ERDA's RECON abstracts (from 1967 to mid1977) and the chemical abstracts condensates called DIALOG (from 1972-1976). However, one unique source which became most valuable to this survey was the compilations of the Radiation Chemistry Data Center of the University of Notre Dame. It was also identified that significant omissions exist in all these three compilations and therefore a major portion of the material used in this survey were derived from routine search done by the investigators.

Among the publications identified, the book by Egorov and Novikow [1] is the only publication of significance on the subject to date. This book covers the progress on the subject up to 1963-64 period and would be a valuable source for further examination. While Egorov"s and Novikov's book has extensively surveyed the Soviet 11terature and publications from Poland, Yugoslavia, Rumania, Hungary and Czechoslovakia, there are some significant omissions in the book on some of the experimental work done in the west. These publications have been identified and incorporated in the present survey. 
Since the publication of Egorov's and Novikov's book, there has been considerable amount of experimental work done. Although this survey has attempted to gather as much of the published experimental data on radiation effects on ion exchange materials, it cannot claim to be all inclusive. For example, we have not been able to translate all the Identified publications in non-English 1anguages. A few publications recognized as significant to this effort were translated for the purpose of this survey.

During this literature survey, we have found that almost a11 the published literature on radiation effects on ion exchangers deal with synthetic organic materials that are of importance to the present day process industry. There is little or no quantitative experimental data on the radiation effects on inorganic ion exchangers that are considered of potential value in processing radioactive materials. It is, however, recognized that the inorganic ion exchangers in general are considerably more resistant to radiation damage and therefore they can be of significant value to nuclear fuel reprocessing and radioactive waste management. Since the necessary experimental work to study the effects of ionizing radiation would require exposures of materials to considerably higher radiation doses, investigators studying the radiation effects on organic materials have not yet actively undertaken these studies.

Our present knowledge on the radiation effects on synthetic organic ion exchangers is better than that of inorganic exchangers. However, the information available today on organic ion exchangers are not adequate to provide meaningful mechanistic interpretations of many of the recognized problems resulting from radiation damages in ion exchangers during process applications. For the present survey, we have assigned mafor importance to synthetfc organic 
exchangers, which are indeed the materials used in the present-day process industry.

This survey has attempted to present objectively all the available facts in an extensive tabulation of experimental data (Tables III and IV) with adequate bibliographical records. Appendices I and II of this report contain general information on the chemical nature of ion exchange resins and the trade names of both organic and inorganic ion exchange materials that are marketed by various manufacturers. In addition, the present knowledge on radiation effects on ion exchange material is sumarized as general rules applicable to most organic ion exchangers. These generalizations should be regarded only as present knowledge. While this survey can definitely add to our present knowledge regarding the radiation chemical changes in ton exchange process materials, it is felt that considerable additional work needs to be done to reliably explain and accurately predict many of the phenomenological observations of experimental studies and process related incidents. 


\section{GENERAL CHARACTERISTICS OF ION EXCHANGE MATERIALS}

The characteristics of ion exchange materials that are of importance in discussing radiation effects are considered here. An attempt will be made in the following section to further elaborate on the influence of radiation-induced chemical changes on these characteristics of ion exchangers.

\section{II.1 Organic and Inorganic Exchangers}

Most of the ion exchange materials that are used (and have the potentials to be used) in radiochemical process applications are synthetic materials. One of the reasons for the preferential use of synthetic materials is the relative ease with which the characteristics of the exchangers can be controlled by appropriate changes in composition and preparative methods. Th1s, in turn, allows for the evaluation and prediction of process characteristics in the use of these materials.

Synthetic organic ion exchangers are high molecular weight polyacids or bases which are virtually insoluble in most aqueous and nonaqueous media. In general, they are cross-linked polymer tridimensional networks to which is attached large polar exchange groups. Since these materials are not individual compounds, their composition and properties are dependent on the nature and purity of the original chemicals from which they are synthesized, and their treatment prior to process applications. Some of the major differences in the properties of synthetic organic ion exchangers produced by various manufacturers as well as the differences between different batches of materials produced by the same manufacturer are accountable by these varlations. Radiation effects on these materlals can also be Influenced, to a limited degree, by the potentials for varlability in different synthetic preparations. In general, radiation effects on synthetic organic ion exchangers can cause irreversible chemical changes 
in their macromolecules due to the rupture of single bonds and the resultant of degradation processes. Also, synthetic cation exchangers contain bonds between functional groups and the polymer matrix. which are relatively unstable. It is these weak linkages that are more 1 lable to succumb to the influence of radiation.

Inorganic ion exchangers, unlike the organic materials, are well defined compounds. In general, they are hydrous oxides. Acidic salts of multivalent metals, salts of heteropolyacids, insoluble ferrodyanides, synthetic aluminosicates or simflar substances of well defined chemical composition. Unlike organlc ion exchangers, these inorganic substrates are highly resistant to radiation exposure. This particular property can be of great value in the nuclear process industry. Two recent review articles $[79,80]$ and a monograph by Amphlett [81] have extensively surveyed the very large number of materials that can be classified as synthetic inorganic ion exchangers. The increasing interest in these materials for potential application in radioactive waste processing is evidenced by several reviews [82-84] by organizations and groups interested in reactor coolant purification and radioactive waste management. However, one of the recognized limitations of inorganic ion exchangers is their relative instability in acid media and their high sensitivity to $\mathrm{pH}$ changes. Therefore, the widespread process applications of inorganic ion exchangers in nuclear fuel reprocessing, reactor coolant maintenance and radioactive waste separations are still awaiting further developments in materials of qualities comparable to the present day synthetic organic ion exchangers. Synthetic organic ion exchangers (resins) are the most widely used and highly developed exchange materials of the day. 
II.2 Classification of Ion Exchangers

Ion exchangers are classifled in several different ways. Some of the major classifications of importance are the following.

II.2.a Classification based on exchangeable species

One of the classifications of ion exchange materials is based on the electrical charge of the chemical spectes they are able to exchange from the aqueous or non-aqueous media. On this basis, ion exchangers are grouped as:

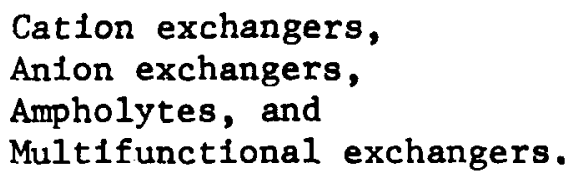

While cation and anion exchangers only sort cations or anions respectively, the ampholytes can act as an anton exchanger or a cation exchanger depending on the conditions under which they are used. Also, there are some ion exchangers that have been synthesized with both cation and anion exchange groups. The number of ampholytes and multifunctional synthetic exchangers are extremely limited and they still remain laboratory curiosities.

\section{II.2.b Classification based on functional groups}

A second, rather arbitrary type, classification of ion exchangers is based on their functional groups and their degree of Ionization (pK yalues). On this basis, Ion exchangers are classifled as strong, intermediate and weak acld/base. type exchangers. Although this classification is fairly arbitrary, it is often used in the ion exchange vocabulary and does serve the purpose of quickly Ident1fying functional groups. Table I summarizes this type of classification and some of the common functional groups of organic ion exchangers used in this grouplng. Polyfunctional (distinct from "multifunctional") Ion exchangers have more than one type of functional groups of the strong/Intermedlate/weak (acid/ base) type. 


\section{Table I}

FUNCTIONAL GROUPS OF SYNTHETIC ORGANIC RESINS

Type of

Resin

Strongly Acid

Intermediate Acid

Weakly Acid

Strongly Basic

Intermediate Base

Weakly Basic
Functiona1 Group

$$
\begin{aligned}
& \text { Nuclear Sulfonic }-\mathrm{SO}_{3} \mathrm{H} \\
& \text { Methylene Sulfonic }-\mathrm{CH}_{2} \mathrm{SO}_{3} \mathrm{H} \\
& \text { Phosphoric - O-P (O)(OH)} 2 \\
& \text { Phosphonic - P(O)(OH) } 2 \\
& \text { Phosphonous - P(O)H(OH) } \\
& \text { Arsonic - As }(\mathrm{O})(\mathrm{OH})_{2} \\
& \text { Carboxylic - COOH } \\
& \text { Phenolic hydroxy1 - } \mathrm{C}_{6} \mathrm{H}_{4} \mathrm{OH} \\
& \text { Sulfhydryl - SH }
\end{aligned}
$$

$$
\begin{aligned}
& \text { Quarternary Ammonium }-\mathrm{N}^{+}\left(\mathrm{CH}_{3}\right)_{3} \\
& -\mathrm{N}^{+}\left(\mathrm{CH}_{2} \mathrm{CH}_{2} \mathrm{OH}\right)_{3} \\
& -\mathrm{N}^{+}\left(\mathrm{CH}_{3}\right)_{2} \mathrm{C}_{2} \mathrm{H}_{4} \mathrm{OH} \\
& \text { Pyridine }-\mathrm{N}^{+} \mathrm{C}_{5} \mathrm{H}_{5} \\
& \text { Quartenary phosphonium } \equiv \mathrm{P}^{+}
\end{aligned}
$$

(Multi-functional consisting of combinations of strongly basic and weakly basic groups)

$$
\begin{aligned}
& \text { p-amino }-\mathrm{NH}_{2} \\
& \text {-amino }=\mathrm{NH} \\
& \text { t-amino } \equiv \mathrm{N}
\end{aligned}
$$

Note: A brief summary of the structural features of ion exchange resins along with descriptions of their chemical make up is included as Appendix I of this report. 


\section{II.2.C Classification based on preparative processes}

Another type of classification for synthetic organic ion exchangers is based on the preparative processes used in the synthesis of the basic macromolecular structure. This classification is considered of significance to radiation induced chemical changes of these ion exchangers. On this basis, the synthetic organic resins are broadly classified as:

\section{Polymerization type, and} Condensation type.

These polymerization and condensation products are resinous materials from which the popular name "resins" for synthetic ion exchangers orfginate. Polymerization type resins are prepared from several molecules of similar (or same) material combining to form larger molecules, while the condensation type of resins are prepared by the combination of several constituents to form a larger molecule with the elimination of smaller molecules like water.

\section{II.2.d Classification based on physical state}

While almost all the ion exchangers considered here are solids, there are a few substances (synthetic organic types) that are classified as ion exchangers In the liquid form. Functionally, the use of these ion exchangers resemble 11quid-1iquid partitioning processes, even though the mechanism of ion partition is considered as ion exchange [85]. At the present time, these liquid ion exchangers are seldom used in fuel reprocessing or radioactive waste separations. II.2.e Classification based on granulation

Ion exchangers are also identifled as granular solids and membrane types. Most of the commercially used resins are the granular types. The membrane type resins are increasingly gaining prominence in several applications. Ion exchange membranes are mechanically rigid films, discs, plates, strips or tubes made of either cation or anion exchange resins. Again, these ion exchange membranes 
have not yet found significant applications in processing involving high level radioactive materials.

\section{II.2.f Classification based on the rigidity of porous structure}

Synthetic polymerization type Ion exchangers are distinguished as ge1type and macroporous (or macrorecticular)-type on the basis of the rigidity of the porous structure of the macromolecule. The macroporous resins are prepared by specialized polymerization techniques with a high degree of cross-linkage. Macroporous resins have an average pore size of $300-700 \mathrm{~A}^{\circ}$ in diameter which are extremely rigid. The conventional gel-type resins have pore structure that are flexible and collapsible [86]. The average surface area per unit weight of macroporous resins is about 400-600 times greater than that of ge1-type resins, $[87,88]$. Unlike gel-type resins, the macroporous resins do not change volume with change in Ionic strength of the solutions. At the present time the macroporous resins are primarily used in organic solvent systems.

\section{3 Properties of Ion Exchangers}

The physicochemical properties of relevance to process applications of commerclally important ion exchange materials are usually found in manufacturer's brochures. An extensive compllation of such data avallable from manufacturers and presented In an IAEA monograph [2] has been adapted as Appendix II of this report. In addition, manufacturer's literature of ten contalns recommendations for column operations and regeneration of the Ion exchangers. However, singularly lacking from these sources is any information regarding the radiation stability of these comerclally used products.

Some of the Important properties of lon exchange materials that are of slgniflcance to radiation effect studies are described below. 


\section{II.3.a Exchange capacity}

One of the most important characteristics of ion exchangers is their exchange capacity, which indicates the amount of ions the material is capable of exchanging under given conditions. By convention [89], it is expressed as milligram equivalent per gram of dry ion exchanger (meq/g). In general, the exchange capacities of strong acid and strong base exchangers remain practically constant over a wide range of $\mathrm{pH}$ values. The exchange capacities of weakly acidic and weakly basic ion exchangers vary greatly depending on the $\mathrm{pH}$ of the media - the weak acid exchangers showing high exchange capacities at higher $\mathrm{pH}$, while the weakly basic exchangers exhibiting higher exchange capacities in solutions of low $\mathrm{pH}$ values.

Since the ion exchange reactions are a result of electrostatic forces, the capacity of ions to be exchanged depends on the relative size of the charge and the radii of the hydrated ions. In dilute solutions, ions with higher valency and higher atomic number (or lower radius of hydration) have greater tendencles for exchange with the solid exchanger. However, this generalization has its limitations, because it is also known that the ability of ions to exchange is dependent on the structure (cross-1inkage) of the sorbent, the $\mathrm{pH}$ of the medium, the ionic concentration of the solution and the temperature of the system.

The effect of ionizing radiations on ion exchangers are often measured in. terms of the change in exchange capacity of the material at vartous exposure rates and under a variety of environmental conditions. This provides a quantitative measure of the rupture of functional groups as a result of radiation induced chemical changes. 


\section{II.3.b Degree of cross-1inking}

Another important characteristic property of organic ion exchangers that has a direct influence on its radiation stability is the degree of cross-linking. This term is used to describe the porosity of an fon exchange particle. Highly cross linked resin can be impermeable to ions of high molecular weight, whereas resins of lower cross-linkage can be quite permeable to the same ions. For synthetic polymerization type resins, cross-linkage is arbitrarily defined as the percentage of divinylbenzene which is used in the preparation of the macromolecular base. Chemicals other than divinylbenzene can also be used to produce cross-1inkage in the resin matrix. For synthetic condensation type resins, the cross-1inkage is determined by the ratio of phenol to substituted phenols used to produce condensation reaction with an aldehyde. It is a common practice to Indicate the cross-1inkage of the resin by an " $x$ " number following the trade name of the resin.

Cross-linkage of synthetic organic exchangers are related to their exchange capacities. In general, on a dry weight basis, the exchange capacity of the resin decreases with increasing cross-linkage. On the other hand, the radiation stability of the resins, in general, tend to increase with increasing cross-linkage. In practice, it is not desirable to increase the cross-linkage of the resins beyond 10 to $15 \%$ because of the increased rate of diminution in exchange capacity beyond this degree of cross-1inkage.

The Influence of Ionizing radiation on resins is to create further crosslinkages in certain instances, while in other cases, radiation exposure causes rupture of cross-linkage resulting in degradation of the ion exchanger. 


\section{II.3.C Swelling and Moisture Content}

When ion exchange sorbents are imnersed in water or in aqueous solutions of an electrolyte, they swell owing to the diffusion of water molecules to the sorbent phase. The tendency of ion exchangers to swell depends on several factors, such as the mesh size of the particle, the degree of cross-inkage of the macromolecule, the rigidity of the spatial network and the degree of dissociation of the functional group. In general, swelling is favored by polar solvents, low degree of cross-linkage of the resin, high capacity of the resin, and low concentration of the external solution.

Ion exchangers placed in liquids continue to swell until an equilibrium condition is reached. The polar and ionic constituents of the resin tend to surround themselves with the solvent and the framework continues to yield until its elastic forces balances the dissolution tendency. The osmotic pressure difference (or "swelling pressure") between the interior and exterior of the resin is often very large, at times greater than a thousand atmospheres [2].

Moisture content of the Ion exchanger furnishes a measure of its waterholding capacity or swelling. Commerclally available ion exchangers always contains a significant amount of molsture. A full elimination of all the water, especially the hydration layer on the polar groups is very difficult without the destruction of the ion exchanger itself.

Radiation chemical effects on ion exchangers are significantly influenced by the presence of moisture within and external to the fon exchanger. The radiolytic products of water often changes the degree and the mechanism of decomposition of the ion exchanger. 


\section{4 Ion Exchange Process}

The fon exchange process is considered a combination of absorption, adsorption and diffusion processes, which is also termed as "sorption" and is similar to chemical double decomposition. Typical ion exchange reactions may be represented as follows:

$$
\begin{aligned}
& \mathrm{R}-\mathrm{H}+\mathrm{X}^{+}+\mathrm{R}-\mathrm{X}+\mathrm{H}^{+} \ldots \text { (cation exchange) } \\
& \mathrm{R}-\mathrm{OH}+\mathrm{Y}^{-}+\mathrm{R}-\mathrm{Y}+\mathrm{OH}^{-} \ldots \text { (anion exchange) }
\end{aligned}
$$

where $\mathrm{R}$ represents the insoluble matrix of the ion exchanger and $\mathrm{X}^{+}$and $\mathrm{Y}^{-}$represent ionic species in solution. The reversal of these processes are employed during the regeneration of depleted ion exchangers. Many factors seem to influence the sorption equilibrium. These include: exchange capacity, degree of cross-linking, concentration of ionic species in solution, ionic valencies, ionic sizes, swelling pressure, rate of diffusion of ions in solution and within the resin, solution temperature and the interactions between mobile and fixed ions in the exchanger. Among these, the most important rate determining factor is the rate of diffusion of lons both in solution and in the resin matrix. A general rule states that ion exchangers tend to exclude strong electrolytes with counter ions (mobile ions) identical to the exchanger counter ions. The degree of this exclusion is enhanced by the low valence of counter ions and high valence of the co-ions.

Radiation effects on ion exchangers and the immediate surroundings of the sorbents influences several of the above mentioned factors, which in turn affect the kinetics of the ion exchange process itself. 


\section{II.5 Chemica1 Composition of Synthetic Organic Ion Exchangers}

Many of the ion exchangers that are often discussed in experimental studies involving radiation chemical effects are synthetic resins of importance to process industry. Resins on which there are meaningful experimental studies reported in the open literature are grouped as cation exchangers and anion exchangers in the tabulations presented in Tables II-A and II-B respectively. These tabulations include a listing of the major constituents of the matrix and the type of process used in their preparation along with the functional groups of the resin. This tabulation uses the trade names of these ion exchangers and. these are arranged in alphabetical order to facilitate cross reference with the radiation effects data presented in the later sections of this report. Further details of the chemical structure of these resins are described in varfous references following the radiation effects data [see Tables III and IV]. 
TABLE IIA

SOME OF THE TYPICAL SYNTHETIC ORGANIC RESINS

(CATION EXCHANGERS)

\section{Trade Name}

Amberlite

IR-120

Amberlite

IR-112

\section{Amberlite}

IR-105

Amberlite

IRC-150

Catex S

Catex F

Catex FN

Dienon SK-1

Dienon SK-100

Dowex 50

Dowex 50W

Dowex 30

Duolite $\mathrm{C}-3$

Duolite $\mathrm{C}-10$

Duolite CS-101

\section{Functional Group (s)}

Nuclear Sulfonic

"

Methylene-sulfonic and phenolic hydroxyl

Carboxylic

Nuclear sulfonic

Methylene-sulfonic and phenolic hydroxyl

Nuclear sulfonic

Nuclear sulfonic

11

11

II

Methylene sulfonic and phenolic hydroxyl

Methylene sulfonic and phenolic hydroxyl

Nuclear sulfonic

Carboxylic
Type of Preparation

Copolymer of styrene and divinyl-benzene (DVB)

"1

Condensation type phenol and formaldehyde

Copolymer of Methyl Methacralate (or methacrylic acid and DVB)

Copolymer of styrene and DVB

Condensation type phenol and formaldehyde

Condenstaion type phenol, formaldehyde and naphthalene

Copolymer of styrene and DVB

11

11

II

Condensation type phenol and formaldehyde

Condensation type phenol and formaldehyde

Condensation type phenol, formaldehyde and naphthalene

Copolymer of acryilc acid and DVB 


\begin{tabular}{|c|c|c|}
\hline Trade Name & Functional Group (s) & Type of Preparation \\
\hline $\mathrm{EO}-7$ & $\begin{array}{l}\text { Nuclear sulfonic and } \\
\text { phenolic hydroxyl }\end{array}$ & $\begin{array}{l}\text { Condensation type phenol, } \\
\text { formaldehyde and hydroquinone }\end{array}$ \\
\hline Imac $C-12$ & Nuclear sulfonic & $\begin{array}{l}\text { Copolymer of styrene and } \\
\text { DVB }\end{array}$ \\
\hline $\mathrm{KB}-6$ & Carboxylic & $"$ \\
\hline $\mathrm{KB}-4$ & $"$ & $\begin{array}{l}\text { Copolymer of methylmethac- } \\
\text { ralate and DVB }\end{array}$ \\
\hline $\mathrm{KB}-4 \mathrm{P}-2$ & $"$ & $"$ \\
\hline $\mathrm{KF}-1$ & Phosphonic & $\begin{array}{l}\text { Copolymer of styrene and } \\
\text { DVB }\end{array}$ \\
\hline KFU & Carboxylic & $\begin{array}{l}\text { Condensation type penoxy- } \\
\text { acetic acid, formaldehyde and } \\
\text { phenol }\end{array}$ \\
\hline KS & Carboxylic & $\begin{array}{l}\text { Copolymer of styrene and } \\
\text { DVB }\end{array}$ \\
\hline $\mathrm{KU}-2$ & Nuclear sulfonic & $\begin{array}{l}\text { Copolymer of styrene and } \\
\text { DVB }\end{array}$ \\
\hline $\mathrm{KU}-4$ & $" 1$ & $\begin{array}{l}\text { Copolymer of acenaphthalene } \\
\text { and DVB }\end{array}$ \\
\hline$K U-5$ & Nuclear sulfonic & $\begin{array}{l}\text { Condensation type formalde- } \\
\text { hyde, and naphthalene sul- } \\
\text { fonic acid }\end{array}$ \\
\hline $\mathrm{KU}-6$ & Nuclear sulfonic & $\begin{array}{l}\text { Condensation type acenaph- } \\
\text { thalene sulfonic acid and } \\
\text { formaldehyde }\end{array}$ \\
\hline $\mathrm{KU}-160$ & " & $\begin{array}{l}\text { Condensation type carbazole } \\
\text { and formaldehyde }\end{array}$ \\
\hline $\mathrm{KU}-1$ & $" 1$ & $\begin{array}{l}\text { Condensation type phenol } \\
\text { and formaldehyde }\end{array}$ \\
\hline $\mathrm{KU}-1 \mathrm{~g}$ & $" 1$ & $"$ \\
\hline Nalcite HCR & $"$ & $\begin{array}{l}\text { Copolymer of styrene and } \\
\text { DVB }\end{array}$ \\
\hline
\end{tabular}


TABLE IIA (Cont'd)

\begin{tabular}{|c|c|c|}
\hline Trade Name & Functional Group (s) & Type of Preparation \\
\hline Permutit Q & Nuclear sulfonic & $\begin{array}{l}\text { Copolymer of styrene and } \\
\text { DVB }\end{array}$ \\
\hline Permutit $\mathrm{H}-70$ & Carboxylic & $\begin{array}{l}\text { Copolymer of methylmetha- } \\
\text { cralate and DVB }\end{array}$ \\
\hline $\mathrm{P}_{3}$ & Nuclear sulfonic & $\begin{array}{l}\text { Condensation type, benzo- } \\
\text { quinone, benzidine disul- } \\
\text { fonic } 2,2^{\prime} \text { or stilbene di- } \\
\text { sulfonic } 2,2^{\prime} \text { acids }\end{array}$ \\
\hline $\mathrm{P}_{4}$ & $"$ & $"$ \\
\hline $\mathrm{PS}_{3}$ & $"$ & $"$ \\
\hline$R F$ & $\begin{array}{l}\text { Phosphonic and phenolic } \\
\text { hydroxyl }\end{array}$ & $\begin{array}{l}\text { Condensation type phenol, } \\
\text { resorcinol and formalde- } \\
\text { hyde or monohydroxy pheny } 1 \\
\text { phosphate and formaldehyde }\end{array}$ \\
\hline $\mathrm{R}_{5} \mathrm{C}$ & $\begin{array}{l}\text { Carboxylic and phenolic } \\
\text { hydroxyl }\end{array}$ & $\begin{array}{l}\text { Condensation type resorcylic } \\
\text { acid and formaldehyde }\end{array}$ \\
\hline SBS-1 & Nuclear sulfonic & $\begin{array}{l}\text { Copolymer of styrene and } \\
\text { butadiene }\end{array}$ \\
\hline SBS-2 & $"$ & $"$ \\
\hline$S D V-3$ & $"$ & $\begin{array}{l}\text { Copolymer of styrene and } \\
\text { DVB }\end{array}$ \\
\hline $\mathrm{SF}$ & Phosphonic & " \\
\hline SG-1 & Carboxylic & $\begin{array}{l}\text { Copolymer of methylmethac- } \\
\text { ralate and ethylene glycol } \\
\text { dimethacralate }\end{array}$ \\
\hline Wolfatit KPS & Nuclear sulfonic & $\begin{array}{l}\text { Copolymer of styrene and } \\
\text { DVB }\end{array}$ \\
\hline Wolfatit $F$ & $"$ & $\begin{array}{l}\text { Condensation type phenol } \\
\text { and formaldehyde }\end{array}$ \\
\hline Wolfatit $F_{2} S$ & $"$ & $"$ \\
\hline Wolfatit $\mathrm{F}_{4} \mathrm{~S}$ & $"$ & $"$ \\
\hline Wolfatit $\mathrm{FF}_{2} \mathrm{~S}$ & $"$ & " \\
\hline
\end{tabular}


TABLE IIA (Cont' $d$ )

\begin{tabular}{|c|c|}
\hline Trade Name & Functional Group (s) \\
\hline Wolfatit $P$ & $\begin{array}{l}\text { Methylene sulfonic and } \\
\text { phenolic hydroxyl }\end{array}$ \\
\hline Wolfatit $\mathrm{CP}$ & Carboxylic \\
\hline Wolfatit C & $\begin{array}{l}\text { Carboxylic and phenolic } \\
\text { hydroxyl }\end{array}$ \\
\hline Wolfatit CN & $"$ \\
\hline Zeocarb-225 & Nuclear sulfonic \\
\hline Zeolex SA & Nuclear sulfonic \\
\hline ZeoRex & $\begin{array}{l}\text { Methylene sulfonic and } \\
\text { phenolic hydroxyl }\end{array}$ \\
\hline Zeolex WA & Carboxylic \\
\hline Zerolitt -225 & Nuclear sulfonic \\
\hline
\end{tabular}

Type of Preparation

$"$

Copolymer of acrylic acid and DVB

Condensation type resoncylic acid and formaldehyde

Condensation type resorcylic acid, phenol and formaldehyde

Copolymer of styrene and DVB

Copolymer of styrene and DVB

Condensation type phenol and formaldehyde

Copolymer of methylmethacrate and DVB

Copolymer of styrene and DVB 
TABLE IIB

SOME OF THE TYPICAL SYNTHETIC ORGANIC RESINS

(ANION EXCHANGERS)

Trade Name

AM

Amberlite
IRA-400
Amberlite
IRA-401
Amberlite
IRA-410
Amberlite
IRA-411
Amberlite
XE-114
Amberlite
IR-45
Amberlite
IR-4B

Amberlite XE-58

AMP

AN-1

AN-9

AN-2F

AN-21

\section{Functional Group(s)}

Trimethyl benzyl ammonium

$"$

"1

Dimethyl hydroxyethyl ammonium

11

11

$\mathrm{P}-$, S- and t- amino

S- and t- amino

11

Pyridine

P- and S- amino

S- and t- amino

"

Trimethy1 benzylammonium

\section{Type of Preparation}

Copolymer of styrene and divinylbenzene (DVB)

"

11

11

11

Condensation type polyethylene, polyamines and epichlorhydrin

Copolymer of styrene and DVB

Condensation type polyethylene, polyamines and phenol

11

Copolymer of styrene, pyridine and DVB

Condensation type melamire and formaldehyde

Condensation type ammonium sulfate, phenol and formaldehyde

Condensation type polyethylene, polyamines and phenol

Copolymer of acenaph thalene and DVB 
TABLE IIB (Cont'd)

\begin{tabular}{|c|c|c|}
\hline Trade Name & Functional Group (s) & Type of Preparation \\
\hline $\mathrm{AN}-25$ & Pyridine derivative & $\begin{array}{l}\text { Copolymer of } 2,5 \text { methyl- } \\
\text { vinyl pyridine with DVB }\end{array}$ \\
\hline $\mathrm{AN}-25 \mathrm{~A}$ & Pyridine derivative & $\begin{array}{l}\text { Copolymer of } 2,5 \text { methyl- } \\
\text { viny } 1 \text { pyridine with DVB } \\
\text { (methylated derivative) }\end{array}$ \\
\hline $\mathrm{ASD}-2$ & Trimethy 1 benzy 1 ammonium & $\begin{array}{l}\text { Copolymer of styrene and } \\
\text { DVB }\end{array}$ \\
\hline ASD-4 & $\begin{array}{l}\text { Dimethyl hydroxyethyl } \\
\text { ammonium }\end{array}$ & $" 1$ \\
\hline $\mathrm{ASD}-3$ & Pyridine & $\begin{array}{l}\text { Copolymer of styrene, py- } \\
\text { ridine and DVB }\end{array}$ \\
\hline AV -16 & $\begin{array}{l}\text { Pyridine and } S-\text { and } t- \\
\text { amino }\end{array}$ & $\begin{array}{l}\text { Condensation type pyridine, } \\
\text { polyethylene, polyamines } \\
\text { and epichlorohydrin }\end{array}$ \\
\hline $\mathrm{AV}-17$ & Trimethyl benzyl ammonium & $\begin{array}{l}\text { Copolymer of styrene and } \\
\text { DVB }\end{array}$ \\
\hline $\mathrm{AV}-27$ & $\begin{array}{l}\text { Dimethyl hydroxyethyl } \\
\text { ammonium }\end{array}$ & $" 1$ \\
\hline $\mathrm{AV}-18$ & Pyridine & $\begin{array}{l}\text { Copolymer of pyridine, sty- } \\
\text { rene and DVB }\end{array}$ \\
\hline AV -19 & Trimethyl benzyl ammonium & $\begin{array}{l}\text { Copolymer of } 2 \text {-viny } 1 \\
\text { napththalene and DVB }\end{array}$ \\
\hline AV-16 & $\begin{array}{l}\text { Pyridine and } S-\text { and } t- \\
\text { amino }\end{array}$ & $\begin{array}{l}\text { Condensation type pyridine, } \\
\text { polyethylene, polyamines } \\
\text { and epichlorohydrin }\end{array}$ \\
\hline Deacidite FF & Trimethyl benzyl ammonium & $\begin{array}{l}\text { Copolymer of styrene and } \\
\text { DVB }\end{array}$ \\
\hline Dienon SA-200 & $"$ & $" 1$ \\
\hline Dienon SA-100 & $\begin{array}{l}\text { Dimethy } 1 \text { hydroxyethy } 1 \\
\text { ammonium }\end{array}$ & $" 1$ \\
\hline Dowe $x-1$ & Trimethyl benzyl ammonium & $" 1$ \\
\hline Dowex-21K & $" 1$ & $"$ \\
\hline Dowex-2 & $\begin{array}{l}\text { Dimethy } 1 \text { hydroxyethy } 1 \\
\text { ammonium }\end{array}$ & $" 1$ \\
\hline
\end{tabular}


TABLE ILB (Cont'd)

\begin{tabular}{|c|c|c|}
\hline Trade Name & Functional Group (s) & Type of Preparation \\
\hline Dowex-3 & $P-, S-$ and $t-$ amino & $\begin{array}{l}\text { Copolymer of styrene and } \\
\text { DVB }\end{array}$ \\
\hline EDE-10 & $" 1$ & $\begin{array}{l}\text { Condensation type poly- } \\
\text { ethylene, polyamines and } \\
\text { epichlorohydrin }\end{array}$ \\
\hline EDE-1OP & $"$ & $"$ \\
\hline Imac $S-4$ & Trimethyl benzyl ammonium & Polyxylalene from p-xylene \\
\hline MMG-1 & P- and S- amino & $\begin{array}{l}\text { Condensation type urea, } \\
\text { melamine, guanidine and } \\
\text { formaldehyde }\end{array}$ \\
\hline Nalcite SAR & $\begin{array}{l}\text { Dimethyl hydroxyethyl } \\
\text { ammonium }\end{array}$ & $\begin{array}{l}\text { Copolymer of stryene and } \\
\text { DVB }\end{array}$ \\
\hline Nalcite WBR & $\mathrm{P}-, \mathrm{S}-$ and $\mathrm{t}-$ amino & " \\
\hline Permutit $S-2$ & $\begin{array}{l}\text { Dimethy } 1 \text { hydroxyethy } 1 \\
\text { ammonium }\end{array}$ & $" 1$ \\
\hline Permutit SK & Pyridine & $\begin{array}{l}\text { Copolymer of pyridine, } \\
\text { styrene and DVB }\end{array}$ \\
\hline Stamex S & t-sulfonium & $\begin{array}{l}\text { Condensation type anisol } \\
\text { and formaldehyde }\end{array}$ \\
\hline Stamex $S-44$ & $"$ & $" 1$ \\
\hline VP-1 & Pyridine (derivative) & $\begin{array}{l}\text { Copolymer of } 2 \text {-methy } 1-5- \\
\text { vinyl-pyridine and DVB }\end{array}$ \\
\hline Wolfatit-SBW & Trimethyl benzyl ammonium & $\begin{array}{l}\text { Copolymer of styrene and } \\
\text { DVB }\end{array}$ \\
\hline $\begin{array}{l}\text { Wolfatit-SBS- } \\
400\end{array}$ & $\begin{array}{l}\text { Dimethyl hydroxyethyl } \\
\text { ammonium }\end{array}$ & $"$ \\
\hline Wolfatit L & $P-, S-$ and $t-$ amino & $\begin{array}{l}\text { Condensation type poly- } \\
\text { ethylene, polyamines and } \\
\text { epichlorhydrin }\end{array}$ \\
\hline Wolfatit-L-150 & $"$ & $"$ \\
\hline
\end{tabular}




\section{TABLE IIB (Cont'd)}

\begin{tabular}{|c|c|c|}
\hline Trade Name & Functional Group (s) & Type of Preparation \\
\hline Wolfatit MD & S- and t- amino & $\begin{array}{l}\text { Condensation type poly- } \\
\text { ethylene, polyamines and } \\
\text { phenol }\end{array}$ \\
\hline Zeolex SB-I & Trimethy1 benzyl ammonium & $\begin{array}{l}\text { Copolymer of styrene and } \\
\text { DVB }\end{array}$ \\
\hline Zeolex SB-II & $\begin{array}{l}\text { Dimethy } 1 \text { hydroxyethy } 1 \\
\text { ammonium }\end{array}$ & " \\
\hline Zeolex WB & $\mathrm{P}-, \mathrm{S}-$ and $\mathrm{t}-$ amino & " \\
\hline Zerolitt FF-IP & Trimethyl benzyl ammonium & $\begin{array}{l}\text { (Macroporous resin) co- } \\
\text { polymer of styrene and DVB }\end{array}$ \\
\hline
\end{tabular}


III. EXPERIMENTAI DATA ON THE EFFECT

OF RADIATION ON SYNTHETIC ION EXCHANGERS

Because of the prevailing importance of synthetic ion exchangers in nuclear process applications, most of the published papers deal with experimental studies using organic resins. At the present time, there are very few studies reporting quantitative data on the effects of radiation on inorganic ion exchange materials. Most of the published material on the effects of ionizing radiation on ion exchangers resins are the results of experiments conducted using miniature scale resin bands exposed to irradiation sources such as $x$-ray machines, ${ }^{60}$ Co $\gamma$-facilities, electron accelerators and in a few instances, research reactors. There have been few reports on direct contact irradiation where radioactive nuclides are sorbed on the resin matrix. These latter types of studies include the use of both $\beta, \gamma$ emitters and $\alpha$-emitters. The available data also include irradiations performed under a variety of environmental conditions on both polymerization and polycondensation type resins. Many of the findings of the radiation degradation studies of resins have been confirmed by others on similar types of resins of different origin. However, disagreements do exist in several areas and some of the reported conclusions remain debatable. The data presented here and in the discussions in the following sections should be viewed with this limitation in mind.

Some of the discrepancies in the reported experimental observations are attributable to insufficiently defined irradiation conditions, difficulties in radiation dosimetry and the existing variations in the overall qualities of similar type of resins from different batches and those produced by different manufacturers. The difficulties in dosimetries is particularly evident in some of the experimental results using reactor irradiations [see Table III]. 
The tabulation presented here is a summary of experimental data from numerous sources. Most of the quantitative data of relevance are from the first fifty references grouped at the beginning of the reference section of this report. Some of the other references cited in this report also contain similar data and the gist of these findings have been incorporated in the discussions in the following sections.

The choice of the format for their tabulation was primarily dictated by the kind of data available from most of the experimental studies. Some of the important experimental parameters and quantitative data that are generally measured with reliability are tabulated. Along with this, approprlate notations have been included to indicate other detalls presented in each of the original studies. The tabulation is broadly divided into two groups - Table III sumarizing the data on synthetic organic cation exchangers and Table IV contains similar data on anion exchanger resins.

The following subsections are brief descriptions of terms and abbreviations used in Tables III and IV. These descriptions are presented to further define these experimental parameters and results more accurately and to bring out the modifications made, if any, in the original data for brevity and consistency.

\section{III.1 Resin (Trade name)}

Here, the word "resin" is used synonymous with organic amorphous materials, which are not true compounds. Synthetic organic ion exchangers are variously referred to as "resins," "Ion exchange resins," or as "synthetic organic resins" in the published literature.

The trade names of commerclally produced ion exchangers are a combination of letters, numbers and words and in general they have no logical relationship 
to the chemical characteristics of the resin. However, these trade names are the only way a particular commercial product is clearly identified and therefore they are part of the vocabulary in the literature. Another puzzling feature of the trade names is that without any substantiable reason, the trade name of particular resin is often changed and several trade names are used to describe the same resin. Adding to the confusion in trade names is the existing practice of resins manufactured by a few major manufacturers being sold under a variety of trade names by the distributors. It is often difficult to get accurate information on these cross-1istings from either the manufacturer or the distributor.

The trade names used in the tabulations are the same identifications used by the authors of these publications. Some of these names are not found in the most recent literature available from U.S. manufacturers, although the same resin is avallable under a different trade name. The manufacturer's pamphlets also contain additional information to identify the trade names with the type of resin, although very few of these brochures provide detailed information on the chemical constituents of the preparation or the structural formula of the product.

\section{III.2 Ionic Form}

The chemical symbols used here refer to the forms in which the ion exchangers were used in the experimental studies. It is generally known that the salt forms of the ion exchangers are more resistant to radiation damage than the $\mathrm{H}^{+}$or $\mathrm{OH}^{-}$forms. In most of the process applications, the ion exchangers are used in the hydrogen and hydroxyl forms. However, during thelr use, the Ion exchanger changes to salt forms and constitute significant part of a column 
by the time the resin bed accumulates increased radiation doses. Salt forms of the resin generally provide a radiation protection effect.

\section{III.3 Exchange Capacity}

This important property of ion exchangers has been described earlier (see Section II.3.a). There are several different ways in which the exchange capacities of ion exchangers are presented in the literature. The exchange capacity of resins tabulated here is the "TOTAL EXCHANGE CAPACITY," also referred to as the "ultimate capacity" [90].

The total exchange capacity (TEC) is the number of chemical equivalents which the resin has available for exchange per unit weight of the dry form of the ion exchanger. The recommendations of the IUPAC Commission on Analytical Nomenclature is to express the exchange capacity of an ion exchanger as milliequivalent per dry gram of resin (meq/g).

The total exchange capacity (TEC) listed in Tables III and IV is different from other exchange capacities mentioned in the literature. TEC should be distinguished from:

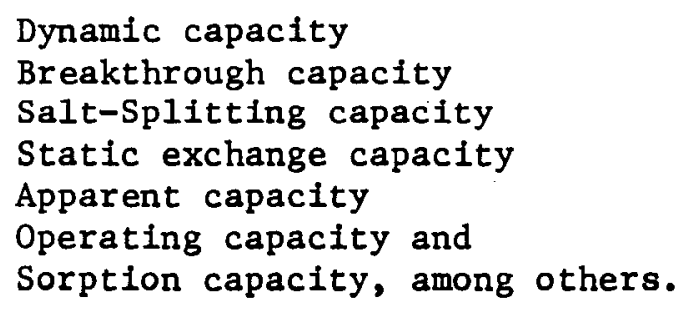

Unlike the various types of exchange capacities mentioned here, the total exchange capacity (TEC) is a constant for a particular exchange material, which shows the number of fixed ionic sites that can enter into an ion exchanger reaction. In Tables III and IV, some of the TEC values reported are in terms of the weight of the matrix (not dry resin). 


\section{III.4 Radiation Source (Dose rate: $\operatorname{Rad} / \mathrm{Hr}$ )}

A variety of radiation sources were used during the experimental studies surveyed here. These include sources of different linear energy transfer (LET) values: amount of energy deposited per unit distance, and sources which are both external and internal to the resin sample. These sources are brlefly 1dentified and where known, the dose rates of the ionizing sources.are included. A11 the reported data have been converted to dose rate as rad/hr for uniformity of presentation.

\section{III.5 Irradiation Condition(s)}

A variety of irradiation conditions have been used in the experimental studies reported because the environmental conditions and the molsture (or electrolyte) content of the resin signlficantly influences the nature and the extent of radiation chemical changes in the ion exchange material. This diverse information is presented in abbreviated form. Further detalls regarding irradiation conditions are described in the original publication(s).

III.6 Integral Dose (Rads)

This is the total dose the resin is reported to have accumulated before its change in properties were measured during the experimental study. Again, for uniformity of presentation, this data has been converted into rad unfts one rad being equivalent to $100 \mathrm{erg}$ of absorbed energy/g or $6.24 \times 10^{13} \mathrm{ev} / \mathrm{g}$ or $10^{-5}$ watt-second/g.

In estimating absorbed radlation doses, most of the experimenters have assumed that the mass absorption coefficients of $x$-rays and $\gamma$-radiations and the LET values for accelerated electrons and radioactive sources emitting $\beta$-particles In the resins are approximately equal to those values in water. This is generally considered a reasonable assumption. 
III.7 Loss of Exchange Capacity (\%)

Most of the reported experimental studies have used the measurement of total exchange capacity of the resin after irradiation as a quantitative measure to determine the changes in ion exchange properties of the resin. This is experimentally achieved by measuring the exchange capacity of the irradiated resin using procedures used to determine total exchange capacity. The difference in exchange capacities are expressed as the percentage of initial capacity (TEC) of the resin. In most instances, on radiation exposure, the exchange capacities of the resins decrease as a result of the rupture of functional groups. However, in certain instances, the TEC has shown net increases due to the formation of new functional groups as a consequence of oxidation of the resin matrix. These gains are reported with a negative sign before the number.

Not all of the experimental studies included here report results similarly. The changes in total exchange capacities are calculated from avallable data wherever possible. At times, the experimenters have chosen to report these changes in exchange capacitles using the dry weight of the irradiated specimen. Since radiation chemical changes also result in weight changes of the resin, an attempt was made to identify this type of data and when adequate information on welght changes were avallable, these results were recalculated in terms of the initial weight of the resin. Thus all of the data reported here are with respect to the Initlal capacity and the initlal weight of the resin.

\section{III.8 G-Value}

By definition, G-value, the radiation chemical yield, is the number of species formed as a result of the absorption of $100 \mathrm{ev}$ of lonizing radiation. In the experimental studies surveyed, G-values have been reported for a varlety of different radiation produced specles. The different G-values that have 
been tabulated without further change in this work are the following:

(i) A term $G_{0}$ is used in some of the data reported by Egorov and Novikov [1]. This denotes the value of G calculated from the inftial part of the kinetic curve. The $G_{0}$ value represents the total amount of the initial functional groups lost per $100 \mathrm{ev}$ of radiation energy absorbed by the ion exchange medium. This parameter indicates that the same resin may have varying radiation chemical resistance which has been confirmed by experimental findings of other workers. The data from Tables 70 and 77 (identified in the last column of the tabulation) from reference [1] are $G_{0}$ values as described above. Most of the other reported values of $G$ are the average value of $G$ considering the integral dose and the net radiation chemical change. If the dependence between radiation chemical change and the dose is linear, $G_{0}$ is proportional to $G$.

(ii) Some of the G-values tabulated are radiation chemical yields estimated during this work from the data provided in the original papers.

(iii) The tabulation also includes G-values reported by the authors for the formation of gases such as $\mathrm{H}_{2}, \mathrm{O}_{2}, \mathrm{SO}_{2}, \mathrm{CO}_{2}$ and $\mathrm{CO}$ and $\mathrm{G}$-values for the formation of sulfate and sulfide lons found in aqueous extracts of 1rradiated resIns. Other forms of G-values reported are for the formation of basic products such as ammonia and $\mathrm{p}-, \mathrm{S}-$ and/or t-amine. An attempt has been made to 1dentify these different forms of reported G-values in the tabulated data.

\section{III.9 Other Parameters Measured}

Because of the limitations of space, this tabulation could not include all the experimental data from original papers. Therefore a summary of varlous other parameters studied during the experimental investigations are indicated by letter symbols. It should be added that these notations do not necessarily 
mean that the original work provided quantitative data on these various parameters. Often, they are qualitative discussions and sometimes pure speculations. The letter designations used in this column of Tables III and IV refers to:
a. weight change
b. swelling/shrinkage or volume change
c. water content
d. solubility
e. oxidizability
f. gas evolution
g. thermal stability
h. products of decomposition
i. formation of lonic species such as $\mathrm{SO}_{4}^{--}, \mathrm{H}^{+}, \mathrm{Cl}^{-}$etc.
j. use of scavenger
k. exchange capacity measurements other than TEC
1. attempt to propose mechanism(s)
m. study of water soluble products, and
n. microscopic examination of resin beads.

\section{10 Reference Number and Notes}

The reference numbers indicated are as listed in the reference section of this report. In addition, when 1t was found necessary, additional notes have been added to further assist the understanding of the tabulated data. 
TARLE III

SUMPAARY OF FXPERIMENTAL DATA ON THF, EFFFCT

F IONIZING, RADIATION ON SYNTHFTIC CATION EXCHANGFR

\begin{tabular}{|c|c|c|c|c|c|c|c|c|c|}
\hline $\begin{array}{c}\text { RESIN } \\
\text { (Trade Name) } \\
\end{array}$ & IONIC FORM & $\begin{array}{l}\text { EXCHANGE } \\
\text { CAPACITY } \\
\text { (men } / g \text { ) } \\
\end{array}$ & $\begin{array}{c}\text { RADIATInN SOURCE } \\
(\mathrm{Rad} / \mathrm{Hr} .)\end{array}$ & $\begin{array}{l}\text { IRRADIATION } \\
\text { CONDITION(S) }\end{array}$ & $\begin{array}{l}\text { INTEGRAL } \\
\text { DOSF. } \\
\text { (RAD) }\end{array}$ & $\begin{array}{c}\text { LOSS OF } \\
\text { EXCHANGE } \\
\text { CAPAC ITY } \\
(\%)\end{array}$ & G VALUE & $\begin{array}{l}\text { OTHER } \\
\text { PARAMETERS } \\
\text { MEASURED } \\
\end{array}$ & $\begin{array}{c}\text { REFERENCE } \\
\text { NUMBER } \\
\text { AND NOTES } \\
\end{array}$ \\
\hline Dowe $x-50$ & $\mathrm{H}^{+}$ & 5.0 & $\begin{array}{l}\gamma \text {-radiation }\left({ }^{60} \mathrm{Co}\right) \\
(0.25-0.6) \times 10^{6}\end{array}$ & Air-dry & $\begin{array}{l}\text { Max. } \\
1.4 \times 1.0^{9}\end{array}$ & - & $2.7-5.3$ & - & [1] Table 70 \\
\hline Dowe $x-50$ & $\mathrm{H}^{+}$ & 3.55 & $\begin{array}{l}\gamma \text {-radtation } \\
(0.19) \times 10^{6}\end{array}$ & Air $\rightarrow$ dry & 3. $3 \times 10^{7}$ & - & 10 & - & [1] Table 70 \\
\hline Dowex-50 & $\mathrm{Na}^{+}$ & 3.55 & $\begin{array}{l}\gamma \text {-rad1at1on } \\
(0.19) \times 10^{6}\end{array}$ & $A i r-d r y$ & $3.3 \times 10^{7}$ & - & 7 & - & [1] Table 70 \\
\hline nowe $x-50$ & $\mu^{+}$ & 5.0 & $\begin{array}{l}\gamma \text {-radlation }\left({ }^{60} \mathrm{Co}\right) \\
\text { and } 144 \mathrm{Ce}-\mathrm{Pr}(0.25 \\
0.6)\end{array}$ & Afr-dry & $\begin{array}{l}\operatorname{Max} .8 \\
9 \times 10^{8}\end{array}$ & - & $1.5-3$ & - & [1] Table 70 \\
\hline Dowex -50 & $\mathrm{H}^{+}$ & 5.0 & $x$-rays $\left(1.12 \times 10^{8}\right)$ & Air-dry & $2.2 \times 10^{9}$ & - & 3. & - & [1] Table 70 \\
\hline Dowe $x-50$ ix 12 & $\mathrm{H}^{+}$ & 5.17 & $\begin{array}{l}\gamma \text {-radlation } \\
\text { (1soton } 1 c)\end{array}$ & Dry & $2.7 \times 10^{8}$ & - & 1.4 & k & (1) Table 70 \\
\hline
\end{tabular}

\begin{tabular}{|c|c|c|c|c|c|c|c|c|c|}
\hline Dowe $x-50 \mathrm{~W} \times 12$ & $\mathrm{H}^{+}$ & 5.17 & $\gamma$-enitter & Swollen in water & $8 \times 10^{7}$ & - & 1.4 & $\mathrm{k}$ & (1) Table 77 \\
\hline howe $x-50 \mathrm{~W} \times 12$ & $\mathrm{H}^{+}$ & - & Y-emitter & Swollen in water & $9 \times 10^{7}$ & - & 0.6 & k & [1] Table 78 \\
\hline Dowex $50 \times 8$ & $\mathrm{H}^{+}$ & 4.80 & y-radiation $\left({ }^{60} \mathrm{Co}\right)$ & Moist, Flowing Water & $0.76 \times 10^{9}$ & 33.8 & 1.0 & $c, k$ & $"$ \\
\hline Dowex $50 \times 12$ & $\mathrm{H}^{+}$ & 4.85 & $"$ & $"$ & $0.77 \times 10^{9}$ & 30.3 & 1.1 & $"$ & $"$ \\
\hline Dowex $50 \times 16$ & $\mathrm{H}^{+}$ & 4.81 & $"$ & $"$ & $0.75 \times 10^{9}$ & 27.9 & 1.2 & $"$ & {$[4][5]$} \\
\hline Dowex $50 \times 2 n$ & $\mathrm{H}^{+}$ & 4.59 & " & $"$ & $0.77 \times 10^{9}$ & 28.8 & 1.6 & $"$ & $"$ \\
\hline Nowex $50 \times 8$ & $\mathrm{H}^{+}$ & 4.97 & $"$ & $"$ & $3.9 \times 10^{9}$ & 62.2 & - & $"$ & {$[4][5]$} \\
\hline Dowex $50 \times 8$ & 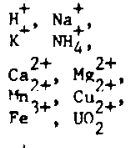 & - & $\begin{array}{l}\text { r-radiation }\left({ }^{60} \mathrm{Co}\right) \\
\left(1.3 \times 10^{5}\right)\end{array}$ & $\begin{array}{l}\text { Varying motsture } \\
\text { contents and ad- } \\
\text { sorbed ions }\end{array}$ & $\begin{array}{l}0 \text { to } \\
1.9 \times 10^{8}\end{array}$ & $0-18$ & - & $b, c, 1, k, 1$ & [10] \\
\hline Dowex $50 \times 2$ & $\mathrm{H}^{+}$ & - & $\begin{array}{l}\text { y-radiation }\left({ }^{60} \mathrm{Co}\right) \\
\left(1.3 \times 10^{5}\right)\end{array}$ & In contact with water & $3.6 \times 10^{7}$ & 15.7 & - & $b, c, i, k, 1$ & {$[10\}$} \\
\hline Dowex $50 \times 2$ & $\mathrm{H}^{+}$ & - & $"$ & $"$ & $"$ & 17.1 & - & $"$ & $"$ \\
\hline Dowex $50 \times 4$ & $\mathrm{H}^{+}$ & - & $"$ & $"$ & $"$ & 6.0 & - & $"$ & $"$ \\
\hline nowex $50 \times 4$ & $\mathrm{H}^{+}$ & - & $"$ & $"$ & $"$ & 6.5 & - & $"$ & $"$ \\
\hline Dowex $50 \times 8$ & $\mathrm{H}^{+}$ & 5.0 & $"$ & $"$ & $"$ & 4.0 & - & $"$ & $"$ \\
\hline Dowex $50 \times 8$ & $H^{+}$ & 5.0 & $"$ & $"$ & $"$ & 4.2 & - & $"$ & $"$ \\
\hline Dowex $50 \times 8$ & $\mathrm{H}^{+}$ & - & $\begin{array}{l}\gamma \text {-radiation }\left({ }^{60} \mathrm{Co}\right) \\
\left(1.3 \times 10^{5}\right)\end{array}$ & $\begin{array}{l}\text { Mo1st (In distilled } \\
\text { water) }\end{array}$ & $6.1 \times 10^{7}$ & 6.8 & - & $b, c, 1, k, 1$ & {$[10]$} \\
\hline Dowe $50 \times 8$ & $\mathrm{Na}^{+}$ & - & $"$ & " & $"$ & 6.7 & - & $"$ & $"$ \\
\hline Dowe $50 \times 8$ & $\mathrm{~K}^{+}$ & - & " & " & $"$ & 6.8 & - & $"$ & $"$ \\
\hline Dowex $50 \times 8$ & $\mathrm{NH}_{4}^{+}$ & - & $"$ & $"$ & $"$ & 6.7 & - & $"$ & " \\
\hline Dowex 50.8 & $\mathrm{Ca}^{2+}$ & - & $"$ & $"$ & $"$ & 6.9 & - & $"$ & $"$ \\
\hline Dowe $\times 50 \times 8$ & $\mathrm{Mp}^{2+}$ & - & $"$ & $"$ & $"$ & 7.2 & - & $"$ & " \\
\hline Dowe $50 \times 8$ & $\mathrm{Mn}^{2+}$ & - & " & $"$ & $"$ & 7.3 & - & $"$ & $"$ \\
\hline Dowex $50 \times 8$ & $\mathrm{Cu}^{2+}$ & - & $"$ & $"$ & $"$ & 0.7 & - & $"$ & $"$ \\
\hline Dowex $51 \times 8$ & $\operatorname{Re}^{3+}$ & - & $"$ & $"$ & " & 0.6 & - & $"$ & $"$ \\
\hline Dowex $50 x^{\AA}$ & $\mathrm{un}_{2}^{2+}$ & - & " & $"$ & $"$ & 0.7 & - & $"$ & " \\
\hline Dowex $50 \times 8$ & $\mathrm{H}^{+}$ & 5.0 & - & $\begin{array}{l}\text { Unhydrous resin and } \\
\text { varylng molsture con- } \\
\text { tent }\left(1-305 \mathrm{H}_{2} \mathrm{O} /\right. \\
\text { meq. resin) }\end{array}$ & $3.6 \times 10^{7}$ & 1.5 to 4.2 & - & $b, c, 1, k, 1$ & {$[10]$} \\
\hline Dowex 50 & $\mathrm{Na}^{+}$ & 3.55 & $\begin{array}{l}\text { y-radiation }\left({ }^{60} \mathrm{Co}\right) \\
\left(2 \times 10^{5}\right)\end{array}$ & $\begin{array}{l}\text { Dry resin } \\
\left.\text { (Dried Ar } 60^{\circ} \mathrm{C}\right)\end{array}$ & $2 \times 10^{5}$ & -0.5 & - & $1, k$ & [12] \\
\hline Dowe x 50 & $\mathrm{Na}^{+}$ & 3.55 & $"$ & $"$ & $3 \times 10^{6}$ & 2.3 & - & $"$ & $"$ \\
\hline Dowex 50 & $\mathrm{Na}^{+}$ & 3.55 & $"$ & $"$ & $4 \times 10^{6}$ & 1.7 & - & $"$ & $"$ \\
\hline Dowex 50 & $\mathrm{Na}^{+}$ & 3.55 & $"$ & $"$ & $3.54 \times 10^{7}$ & 6.5 & - & $"$ & $"$ \\
\hline Dowex $5 n$ & $\mathrm{H}^{+}$ & 3.55 & $\begin{array}{l}\text { Y-radiation }\left({ }^{60} \mathrm{Co}\right) \\
\left(2 \times 10^{5}\right)\end{array}$ & Dry resin & $\begin{array}{l}0.02 \text { to } \\
3.5 \times 10^{7}\end{array}$ & $\begin{array}{l}-3.1 \text { co } \\
10.4\end{array}$ & - & $1, k$ & {$[12]$} \\
\hline
\end{tabular}


TABLE II I (Cont 'd)

\begin{tabular}{|c|c|c|c|c|c|c|c|c|c|}
\hline $\begin{array}{c}\text { RESIN } \\
\text { (Trade Name) } \\
\end{array}$ & IONIC FORM & $\begin{array}{l}\text { EXCHANCE } \\
\text { CAPACITY } \\
\text { (meq } / 8) \\
\end{array}$ & $\begin{array}{l}\text { RADIATION SNURCE } \\
(\mathrm{Rad} / \mathrm{Hr} .)\end{array}$ & $\begin{array}{l}\text { IRRADIATION } \\
\text { CONDITION (S) }\end{array}$ & $\begin{array}{c}\text { INTFGRAL. } \\
\text { DOSF } \\
\text { (RAD) } \\
\end{array}$ & $\begin{array}{c}\text { LOSS OF } \\
\text { rXCHANGE } \\
\text { CAPACITY } \\
(\%) \\
\end{array}$ & G VALUE & $\begin{array}{l}\text { OTHER } \\
\text { PARAMETERS } \\
\text { MEASURED } \\
\end{array}$ & $\begin{array}{l}\text { REFERENCE } \\
\text { NUMBER } \\
\text { AND NOTES } \\
\end{array}$ \\
\hline Dowex $50 \mathrm{w} \times 12$ & $\mathrm{H}^{+}$ & 5.17 & $\begin{array}{l}\text { Y-radiation (Mixed } \\
\text { flssion products } \\
\text { from spent reactor } \\
\text { fuels) }\left(3 \times 10^{5}\right)\end{array}$ & $\begin{array}{l}\text { Imersed in water } \\
(44.4 \% \text { water })\end{array}$ & $0.88 \times 10^{8}$ & 1.5 & - & $\begin{array}{l}a, b, c, h, 1, \\
k, m\end{array}$ & [22] \\
\hline Dowex $50 \mathrm{w} \times 2$ & $\mathrm{H}^{+}$ & 5.17 & $"$ & $\begin{array}{l}\text { Dry }(44.67 \text { water) } \\
\left.\text { (Dried at } 110^{\circ} \mathrm{C}\right)\end{array}$ & $0.76 \times 10^{8}$ & 3.3 & - & $"$ & $"$ \\
\hline Dowe $50 \mathrm{Wx} \times 2$ & $\mathrm{H}^{+}$ & 5.17 & $"$ & Dry ( 482 water) & $1.10 \times 10^{8}$ & -0.3 & - & $"$ & $"$ \\
\hline Dowex $50 \mathrm{Wx} 12$ & $\mathrm{H}^{+}$ & 5.17 & $"$ & Dry $(55.9 \%$ water $)$ & $2.67 \times 10^{8}$ & -0.3 & - & $"$ & $"$ \\
\hline Dowex $501 \mathrm{v} \times 4$ & $\mathrm{H}^{+}$ & 5.35 & $\begin{array}{l}\text { Y-radiation }\left({ }^{60} \mathrm{Co}\right) \\
\left(1.25 \times 10^{6}\right)\end{array}$ & $\begin{array}{l}\text { Air-dried } \\
\quad(67.9 \% \text { water })\end{array}$ & $0.88 \times 10^{8}$ & 5.4 & - & $\begin{array}{l}a, b, c, f, i, \\
k, n\end{array}$ & [23] \\
\hline$"$ & $"$ & $"$ & $"$ & $"(687, \quad ")$ & $1.17 \times 10^{8}$ & 10.8 & - & $"$ & $"$ \\
\hline$"$ & $"$ & $"$ & $"$ & $"(68.4 z ")$ & $2.93 \times 10^{8}$ & 21.1 & - & $"$ & $"$ \\
\hline$"$ & $"$ & $"$ & $"$ & $"(68.0 \% ")$ & $3.59 \times 10^{8}$ & 24.1 & - & $"$ & $"$ \\
\hline$"$ & $"$ & $"$ & $"$ & $"(66.3 \% ")$ & $5.12 \times 10^{8}$ & 29.5 & - & $"$ & $"$ \\
\hline$"$ & $"$ & $"$ & $"$ & $"(61.8 \% ")$ & $7.15 \times 10^{8}$ & 37.7 & - & $"$ & $"$ \\
\hline nowex $501 \times 4$ & $\mathrm{H}^{+}$ & 5.35 & $\begin{array}{l}\text { Y-radiation } \\
\left(1.25 \times 10^{6}\right)\end{array}\left({ }^{60} \mathrm{Co}\right)$ & $\begin{array}{l}\text { In } 0.1 \mathrm{~N} \mathrm{HCl} \\
(68.6 \% \text { water })\end{array}$ & $1.17 \times 10^{8}$ & 9.4 & - & $\begin{array}{l}a, b, c, f, 1 \\
k, n\end{array}$ & [23] \\
\hline$"$ & $"$ & $"$ & $"$ & $"(69.6 z ")$ & $1.77 \times 10^{8}$ & 12.5 & - & $"$ & $"$ \\
\hline$"$ & $"$ & $"$ & $"$ & $"(70.37 \quad ")$ & $2.11 \times 10^{8}$ & 16.6 & - & $"$ & $"$ \\
\hline$"$ & $"$ & $"$ & $"$ & $"(69.47 ")$ & $3.00 \times 10^{8}$ & 21.9 & - & $"$ & $"$ \\
\hline$"$ & $"$ & $"$ & $"$ & $"(69.6 \% ")$ & $3.59 \times 10^{8}$ & 25.2 & - & $"$ & $"$ \\
\hline$"$ & " & $"$ & $"$ & $"(67.97 \quad)$ & $5.12 \times 10^{8}$ & 30.5 & - & $"$ & $"$ \\
\hline Dowex 50w 4 & $\mathrm{Na}^{+}$ & 5.35 & $\begin{array}{l}\text { r-radiation }\left({ }^{60} \mathrm{Co}\right) \\
\left(1.25 \times 10^{6}\right)\end{array}$ & $\begin{array}{l}\text { A1r-dried State } \\
\quad(69.4 \% \text { water })\end{array}$ & $1.77 \times 10^{8}$ & 12.2 & - & $\begin{array}{l}a, b, c, f, 1 \\
k, n\end{array}$ & [23] \\
\hline$"$ & $"$ & $"$ & " & $"(68.37 ")$ & $3.85 \times 10^{8}$ & 21.5 & - & " & " \\
\hline " & $"$ & $"$ & $"$ & $"(65.5 \% ")$ & $6.32 \times 10^{8}$ & 27.5 & - & $"$ & $"$ \\
\hline Dowex $50 w \times 4$ & $\mathrm{Na}^{+}$ & 5.35 & $\begin{array}{l}\gamma \text {-radiation }\left({ }^{60} \mathrm{Co}\right) \\
\left(1.25 \times 10^{6}\right)\end{array}$ & $\begin{array}{l}\text { In } 0.1 \mathrm{~N} \mathrm{HCl} \\
(70.2 \pi \text { water })\end{array}$ & $1.77 \times 10^{8}$ & 14.0 & - & $\begin{array}{l}a, b, c, f, i \\
k, n\end{array}$ & [23] \\
\hline " & $"$ & $"$ & $1 t$ & $"(69.47 ")$ & $3.85 \times 10^{8}$ & 21.9 & - & $"$ & $"$ \\
\hline$"$ & $"$ & $"$ & $"$ & $"(71.3 \% ")$ & $6.32 \times 10^{8}$ & 26.9 & - & $"$ & $"$ \\
\hline Dowex $50 \mathrm{w} \times 4$ & $"$ & $"$ & $\begin{array}{l}\text { a-radiation }\left(2^{241} \mathrm{Am}\right. \\
\text { adsorbed on the } \\
\text { resin })\left(3.85 \times 10^{5}\right)\end{array}$ & $\begin{array}{l}\text { In } \begin{array}{l}0.1 \mathrm{~N} \mathrm{HCl} \\
(69.5 \% \text { water })\end{array}\end{array}$ & $1.29 \times 10^{8}$ & 27.1 & - & $b, c, k, m$ & {$[25][27]$} \\
\hline$"$ & $"$ & $"$ & $"$ & $"(76.37 \quad ")$ & $1.94 \times 10^{8}$ & 33.8 & - & $"$ & $"$ \\
\hline$"$ & $"$ & $"$ & $"$ & $"(92.0 \% ")$ & $3.79 \times 10^{8}$ & 81.3 & - & $"$ & $\begin{array}{l}\text { Resin loaded } \\
\text { to } 10 \% \text { ca- } \\
\text { pacity dur- } \\
\text { ing these ir- } \\
\text { radiations }\end{array}$ \\
\hline$"$ & $"$ & $"$ & $"$ & $"(93.58 ")$ & $4.34 \times 10^{8}$ & 84.4 & - & $"$ & $"$ \\
\hline$"$ & $"$ & $"$ & $"$ & $"(94.87 ")$ & $4.80 \times 10^{8}$ & 88.8 & - & $"$ & $"$ \\
\hline$"$ & $"$ & $"$ & $"$ & $"(96.62 \quad ")$ & $5.45 \times 10^{8}$ & 88.0 & - & $"$ & $"$ \\
\hline " & $"$ & $"$ & $"$ & $"(92.58 \quad ")$ & $6.47 \times 10^{8}$ & 83.0 & - & $"$ & $"$ \\
\hline Dowex SOWx 4 & $\mathrm{H}^{+}$ & 5.35 & $\begin{array}{l}\text { a-radiacion }\left({ }^{241} \mathrm{Am}\right. \\
\text { adsorbed on the } \\
\text { resin) }\left(3.65 \times 10^{6}\right)\end{array}$ & $\begin{array}{l}\text { In } 0.1 \mathrm{~N} \mathrm{HC1} \\
(63.42 \text { water })\end{array}$ & $0.88 \times 10^{8}$ & 28.8 & - & $b, c, k, w$ & {$[25][27]$} \\
\hline$"$ & $"$ & $"$ & $"$ & $"(61.3 \% ")$ & $1.76 \times 10^{8}$ & -6.4 & - & $"$ & $"$ \\
\hline$"$ & $"$ & $"$ & $"$ & $"(69.97 \quad ")$ & $2.63 \times 10^{8}$ & 18.7 & - & $"$ & $\begin{array}{l}\text { Resin loaded } \\
\text { to } 100 z \text { ca- } \\
\text { pacity dur- } \\
\text { Ing these } \\
\text { Irradiations }\end{array}$ \\
\hline$"$ & $"$ & $"$ & $"$ & $"(69.72 ")$ & $4.25 \times 10^{8}$ & 15.1 & - & $"$ & $"$ \\
\hline$"$ & $"$ & $"$ & $"$ & $"(58.62 ")$ & $5.27 \times 10^{8}$ & -4.2 & - & $"$ & $"$ \\
\hline$"$ & $"$ & $"$ & $"$ & $"(71.97 \quad)$ & $6.18 \times 10^{8}$ & 22.3 & - & $"$ & $"$ \\
\hline
\end{tabular}




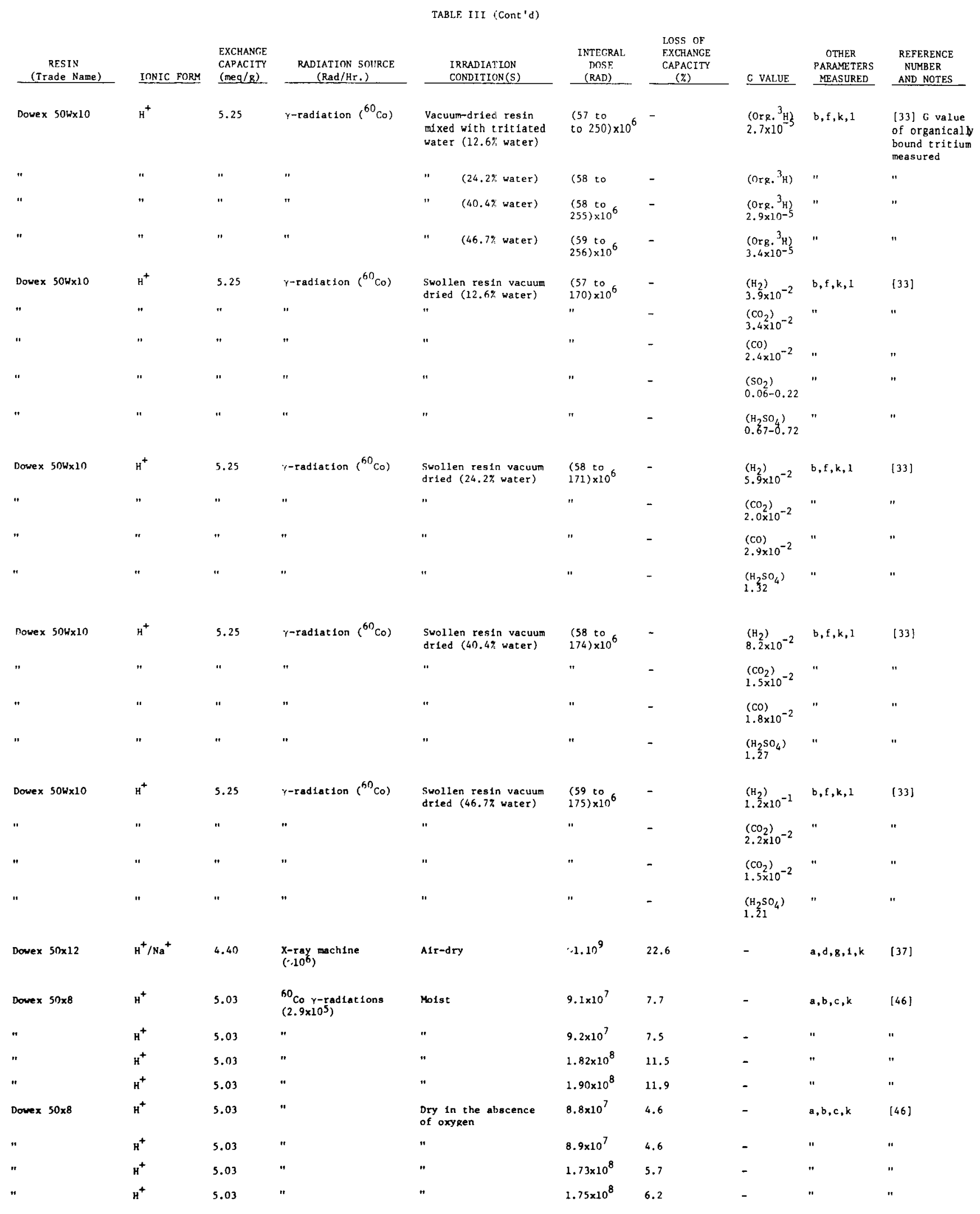




\begin{tabular}{|c|c|c|c|c|c|c|c|c|c|}
\hline \multirow{2}{*}{$\begin{array}{c}\text { RESIN } \\
\text { (Trade Name) } \\
\end{array}$} & & \multirow[b]{2}{*}{ G VALUE } & \multirow[b]{2}{*}{$\begin{array}{c}\text { OTHER } \\
\text { PARAMETERS } \\
\text { MEASURED } \\
\end{array}$} & \multirow[b]{2}{*}{$\begin{array}{c}\text { REFERENCE } \\
\text { NUMBER } \\
\text { AND NOTES } \\
\end{array}$} \\
\hline & $\underline{\text { IONIC FORM }}$ & $\begin{array}{l}\text { EXCHANGE } \\
\text { CAPACITY } \\
\text { (meq } / \mathrm{g}) \\
\end{array}$ & $\begin{array}{c}\text { RADIATION SOURCE } \\
\text { (Rad/Hr.) }\end{array}$ & $\begin{array}{l}\begin{array}{l}\text { IRRADIATION } \\
\text { CONDITION(S) }\end{array} \\
\end{array}$ & $\begin{array}{c}\text { INTEGRAL } \\
\text { DOSF } \\
\text { (RAD) } \\
\end{array}$ & $\begin{array}{l}\text { LOSS OF } \\
\text { EXCHANGF } \\
\text { CAPACTYY } \\
(\%) \\
\end{array}$ & & & \\
\hline Dowex 50 wx 10 & $\mathrm{H}^{+}$ & 5.25 & $\begin{array}{l}{ }^{60} \mathrm{Co} \gamma \text {-radiations } \\
\left(2.5 \times 10^{5}\right)\end{array}$ & Dry & $\begin{array}{l}(0.5 \text { to } \\
2.0) \times 10^{8}\end{array}$ & - & $\begin{array}{l}\left(\mathrm{H}_{2}\right) \\
0.026\end{array}$ & $\mathrm{c}, \mathrm{f}, \mathrm{h}, \mathrm{k}$ & [47] \\
\hline$"$ & " & $"$ & $"$ & $"$ & $"$ & - & $\begin{array}{l}\left(\mathrm{CO}_{2}\right) \\
3.035\end{array}$ & $"$ & $"$ \\
\hline " & $"$ & " & " & $"$ & $"$ & - & $\begin{array}{l}(\mathrm{CO}) \\
0.009\end{array}$ & $"$ & $"$ \\
\hline " & $"$ & $"$ & $"$ & $"$ & $"$ & - & $\begin{array}{l}\left(\mathrm{SO}_{2}\right) \\
0.87\end{array}$ & " & $\begin{array}{l}\mathrm{G}\left(\mathrm{SO}_{2} \text { is the }\right. \\
\text { initial } \\
\text { value for } \\
\text { lowest dose }\end{array}$ \\
\hline Dowex 50 Wx10 & $\mathrm{Li}^{+}$ & 5.25 & $"$ & Dry & $"$ & - & $\begin{array}{l}\left(\mathrm{H}_{2}\right) \\
0.001\end{array}$ & " & $"$ \\
\hline$"$ & " & $"$ & $"$ & $n$ & $"$ & - & $\begin{array}{l}\left(\mathrm{CO}_{2}\right) \\
0.007\end{array}$ & $"$ & $"$ \\
\hline " & $"$ & $"$ & $"$ & $"$ & $"$ & - & $\begin{array}{l}(\mathrm{CO}) \\
0.002\end{array}$ & $"$ & $"$ \\
\hline$"$ & " & " & " & " & " & - & $\begin{array}{l}\left(\mathrm{SO}_{2}\right) \\
0.26\end{array}$ & $"$ & " \\
\hline Dowex $50 \mathrm{~W} \times 10$ & $\mathrm{H}^{+}$ & 5.25 & " & $\begin{array}{l}6 \text { moles of } \mathrm{H}_{2} \mathrm{O} / \mathrm{eq} \text {. of } \\
\text { resin }\end{array}$ & $"$ & - & $\begin{array}{l}\left(\mathrm{H}_{2}\right) \\
0.095\end{array}$ & " & " \\
\hline$"$ & $"$ & " & $"$ & $"$ & $"$ & - & $\begin{array}{l}\left(\mathrm{CO}_{2}\right) \\
0.019\end{array}$ & $"$ & $"$ \\
\hline$"$ & $"$ & $"$ & $"$ & $"$ & $"$ & - & $\begin{array}{l}(c 0) \\
0.027\end{array}$ & $"$ & $"$ \\
\hline$"$ & $"$ & $"$ & $"$ & $"$ & $"$ & - & $\begin{array}{l}\left(\mathrm{SO}_{2}\right) \\
0.006\end{array}$ & $"$ & $"$ \\
\hline Dowex $50 \mathrm{w} \times 10$ & $\mathrm{Li}^{+}$ & 5.25 & $"$ & $\begin{array}{l}5.1 \text { moles of } \mathrm{H}_{2} \mathrm{O} / \mathrm{eq} \text {. } \\
\text { of resin }\end{array}$ & " & - & $\begin{array}{l}\left(\mathrm{H}_{2}\right) \\
0.11\end{array}$ & $"$ & $"$ \\
\hline$"$ & " & " & $"$ & $"$ & $"$ & - & $\begin{array}{l}\left(\mathrm{CO}_{2}\right) \\
0.008\end{array}$ & $"$ & " \\
\hline$"$ & $"$ & $"$ & $"$ & $"$ & $"$ & - & $\begin{array}{l}(c 0) \\
0.019\end{array}$ & $n$ & " \\
\hline " & $"$ & $"$ & $n$ & $n$ & $"$ & - & $\begin{array}{l}\left(\mathrm{SO}_{2}\right) \\
0.50\end{array}$ & " & $"$ \\
\hline Dowex 50 Wx10 & $\mathrm{H}^{+}$ & 5.25 & " & $\begin{array}{l}41 \text { moles of } \mathrm{H}_{2} \mathrm{O} / \mathrm{eq} \text {. of } \\
\text { resin }\end{array}$ & $"$ & - & $\begin{array}{l}\left(\mathrm{H}_{2}\right) \\
1.7\end{array}$ & " & " \\
\hline & & & & & & & $\begin{array}{l}\left(\mathrm{CO}_{2}\right) \\
0.41\end{array}$ & " & " \\
\hline Dowex $50 \% \times 10$ & Lt & 5.25 & $"$ & $\begin{array}{l}24 \text { moles of } \mathrm{H}_{2} \mathrm{O} \text { eq. of } \\
\text { Tesin }\end{array}$ & $"$ & - & $\begin{array}{l}\left(\mathrm{H}_{2}\right) \\
1.3\end{array}$ & " & $"$ \\
\hline & & & & & & & $\begin{array}{l}\left(\mathrm{CO}_{2}\right) \\
0.16\end{array}$ & $"$ & $"$ \\
\hline Dowex $50 \times 4$ & $\mathrm{H}^{+}$ & 5.0 & $\begin{array}{l}{ }^{60} \text { Co } \gamma \text {-radiations } \\
\left(2.5 \times 10^{5}\right)\end{array}$ & Wet & $9.1 \times 10^{7}$ & 5.0 & - & $a, b, c, k$ & [46] \\
\hline$"$ & $\mathrm{H}^{+}$ & 5.0 & $"$ & $"$ & $1.82 \times 10^{8}$ & 10.0 & - & $"$ & $"$ \\
\hline Dowex $50 \times 4$ & $\mathrm{H}^{+}$ & 5.0 & " & Dry & $8.5 \times 10^{7}$ & 2.4 & - & $"$ & $"$ \\
\hline$"$ & $\mathrm{H}^{+}$ & 5.0 & $"$ & $"$ & $1.66 \times 10^{8}$ & 4.6 & - & $"$ & $"$ \\
\hline Amber11te IR-120 & $\mathrm{H}^{+}$ & 5.0 & $\begin{array}{l}{ }^{182} \mathrm{Ta} \text { and } \\
\text { radiations }\end{array}$ & Ail $r-d r y$ & $1.9 \times 10^{8}$ & - & 1.6 & - & [1] Table 70 \\
\hline Amber1ite IR-120 & $\mathrm{H}^{+}$ & 5.0 & $x$-rays $\left(1.12 \times 10^{8}\right)$ & Al $r-d r y$ & $2.2 \times 10^{9}$ & - & 1.2 & - & [1] Table 70 \\
\hline Amberlite IR-120 & $\mathrm{H}^{+}$ & 5.0 & $\begin{array}{l}{ }^{182} \text { Ta and }{ }^{60} \mathrm{Co} \\
\text { radiations }\end{array}$ & Molst & $1.9 \times 10^{8}$ & - & 1.6 & k & (1) Table 77 \\
\hline Amber11te IR-12n & $\underline{p}^{+}$ & 4.5 & $\begin{array}{l}B \text { and } Y \text {-radiations } \\
\text { (activetion and f1s- } \\
\text { sion products) } \\
\left(1.4 \times 10^{7}\right)\end{array}$ & $\begin{array}{l}\text { Mo1st (on-1ine use in } \\
\mathrm{D}_{2} \mathrm{O} \text { moderator clesnup } \\
\text { system) }\end{array}$ & $\begin{array}{l}\text { up to } \\
1.5 \times 10^{8}\end{array}$ & 20.5 to 40 & - & $k, m$ & [13] [15] \\
\hline Amber11te $I R-112$ & $\mathrm{H}^{+}$ & 5.0 & $\begin{array}{l}.182 \mathrm{Ta} \text { and } \\
\text { radiations }\end{array}$ & A1r $-\mathrm{d} r y$ & $1.9 \times 10^{8}$ & - & 1.6 & - & [1] Table 70 \\
\hline Amber lite IR-112 & $\mathbf{H}^{+}$ & 5.0 & $"$ & Motst & $1.9 \times 10^{8}$ & - & 1.6 & - & [1] Table 77 \\
\hline Amberlite IR-105 & $\mathbf{H}^{+}$ & 2.7 & $"$ & A1r-dry & $9 \times 10^{8}$ & - & 0.07 & - & [1] Table 70 \\
\hline Amberlite IRC-50 & $\mathrm{H}^{+}$ & 10.0 & $\begin{array}{l}x-\text { ray un } 1 t \\
\left(1.12 \times 10^{8}\right)\end{array}$ & Alr-dry & $2.2 \times 10^{9}$ & - & 2.7 & - & [1] Table 70 \\
\hline Amber11te 200 & $\mathrm{H}^{+}$ & 5.00 & $"$ & Mo1st, flowing water & $0.97 \times 10^{9}$ & 34.0 & 1.2 & $c, k$ & {$[4][5]$} \\
\hline
\end{tabular}




\begin{tabular}{|c|c|c|c|c|c|c|c|c|c|}
\hline $\begin{array}{c}\text { RESIN } \\
\text { (Trade Name) } \\
\end{array}$ & IONIC FORM & $\begin{array}{l}\text { EXCHANGE } \\
\text { CAPACITY } \\
(\operatorname{meq} / g) \\
\end{array}$ & $\begin{array}{c}\begin{array}{c}\text { RADIATION SNURCE } \\
(\mathrm{Rad} / \mathrm{Hr} .)\end{array} \\
\end{array}$ & $\begin{array}{l}\text { IRRADIATION } \\
\text { CONDITION(S) } \\
\end{array}$ & $\begin{array}{c}\text { INTEGRAL } \\
\text { DOSE } \\
\text { (RAD) } \\
\end{array}$ & $\begin{array}{c}\text { LOSS OF } \\
\text { EXCHANGE } \\
\text { CAPACTYY } \\
(\%) \\
\end{array}$ & G VALUE & $\begin{array}{c}\text { OTHER } \\
\text { PARAMETERS } \\
\text { MEASURED } \\
\end{array}$ & $\begin{array}{c}\text { REFERENCE } \\
\text { NUMBER } \\
\text { AND NOTES } \\
\end{array}$ \\
\hline Amber11te $I R-12 n$ & - & 4.97 & $\begin{array}{l}{ }^{182} \mathrm{Ta} \\
(4 \text { to } \\
\left(4.2 \times 10^{5}\right)\end{array}$ & $\begin{array}{l}\text { Dried at } 60^{\circ} \mathrm{C} \text { for } 48 \\
\text { hrs }\end{array}$ & $1.68 \times 10^{6}$ & -3.2 & - & $c, h, 1, k$ & {$[34][35][36]$} \\
\hline Amberlite IR-120 & - & 4.97 & " & " & $1.72 \times 10^{7}$ & 8.0 & - & " & " \\
\hline Amberlite $I R-12 n$ & - & 4.97 & $"$ & Wet (f1ltered) & $2.13 \times 10^{6}$ & 3.0 & - & $"$ & $"$ \\
\hline Amberlite $I R-120$ & - & 4.97 & " & $"$ & $1.73 \times 10^{7}$ & 7.4 & - & " & $"$ \\
\hline Amberlite IR-120 & $\mathrm{H}^{+} / \mathrm{Na}^{+}$ & 4.15 & $x$-ray machine & Adr-dry & $21 \times 10^{9}$ & 9.4 & - & $a, d, 8,1, k$ & [37] \\
\hline Amberl1te IR-112 & - & 5.19 & $\begin{array}{l}182 \text { Ta Y-radiations } \\
\left(4.0 \text { to } 4.2 \times 10^{5}\right)\end{array}$ & Dry & $1.68 \times 10^{6}$ & 2.9 & - & $c, h, 1, k$ & {$[34][35][36]$} \\
\hline$"$ & - & 5.19 & $"$ & " & $1.72 \times 10^{7}$ & 5.5 & - & " & $"$ \\
\hline$"$ & - & 5.19 & $\begin{array}{l}{ }^{60} \mathrm{Co} \gamma \text {-radiations } \\
\left(4.2 \times 10^{5}\right)\end{array}$ & $"$ & $1.71 \times 10^{8}$ & -2.1 & - & " & $"$ \\
\hline Amberlite IR-112 & - & 5.19 & $\begin{array}{l}{ }^{182} \text { Ta } \gamma \text {-radiation } \\
\left(4.0 \text { to } 4.2 \times 10^{5}\right)\end{array}$ & Wet (filtered) & $2.13 \times 10^{6}$ & 1.3 & - & $"$ & " \\
\hline$"$ & - & 5.19 & " & " & 1. $23 \times 10^{7}$ & 0.8 & - & " & $"$ \\
\hline Amber1ite IR-112 & - & 5.19 & $\begin{array}{l}{ }^{60} \mathrm{Co} \text { rrad1ations } \\
\left(4.2 \times 10^{5}\right)\end{array}$ & " & $1.83 \times 10^{8}$ & -7.5 & - & " & $"$ \\
\hline Amberlite IR-10n & - & 5.38 & $\begin{array}{l}{ }^{182} \mathrm{Ta} \text { Y-radiations } \\
\left(4.0 \text { to } 4.2 \times 10^{5}\right)\end{array}$ & Dry & $1.68 \times 10^{6}$ & 2.8 & - & $c, h, 1, k$ & {$[34][35][36]$} \\
\hline$"$ & - & $5.3 B$ & $"$ & $"$ & $1.72 \times 10^{7}$ & 0.6 & - & $"$ & $"$ \\
\hline$"$ & - & 5.38 & $\begin{array}{l}{ }^{60} \mathrm{Co} \gamma \text { r-radiations } \\
\left(4.2 \times 10^{5}\right) \\
(4)\end{array}$ & " & $1.71 \times 10^{8}$ & 12.3 & - & " & $"$ \\
\hline Amber11te IR-100 & - & 5.38 & $\begin{array}{l}{ }^{182} \text { Ta r-radiations } \\
\left(4.0 \text { to } 4.2 \times 10^{5}\right)\end{array}$ & Wet (f11tered) & $2.13 \times 10^{6}$ & 2.4 & - & " & $"$ \\
\hline$"$ & - & 5.38 & $"$ & $"$ & $1.73 \times 10^{7}$ & 1.7 & - & $"$ & $"$ \\
\hline$"$ & - & 5.38 & $\begin{array}{l}{ }^{60} \operatorname{co} \gamma \text {-radiat1ons } \\
\left(4.2 \times 10^{5}\right)\end{array}$ & $"$ & $1.83 \times 10^{8}$ & 15.8 & - & $"$ & $"$ \\
\hline Amberlite XE-111 & - & 6.03 & $\begin{array}{l}{ }^{182} \text { Ta }_{\mathrm{A}} \gamma-\mathrm{radiat} \text {-rons } \\
\left(4,0-4.2 \times 10^{5}\right)\end{array}$ & Dry. & $1.68 \times 10^{6}$ & -4.0 & - & $c, h, 1, k$ & {$[34][35][36]$} \\
\hline " & - & 6.03 & $"$ & $"$ & $1.72 \times 10^{7}$ & -1.2 & - & $"$ & $"$ \\
\hline$"$ & - & 6.03 & $\begin{array}{l}{ }^{60} \text { Co } \gamma \text {-radiations } \\
\left(4.2 \times 10^{5}\right)\end{array}$ & $"$ & $1.71 \times 10^{8}$ & -9.5 & - & $"$ & " \\
\hline Amber11te XE-111 & - & 6.03 & $\begin{array}{l}{ }^{182} \text { Ta r-radiatjons } \\
\left(4.0 \text { to } 4.2 \times 10^{5}\right)\end{array}$ & Wet (f11tered) & $2.13 \times 10^{6}$ & 1.3 & - & $c, h, 1, k$ & {$[34][35][36]$} \\
\hline " & - & 6.03 & $"$ & $"$ & $1.73 \times 10^{\prime}$ & 1.0 & - & $"$ & " \\
\hline$"$ & - & 6.03 & $\begin{array}{l}{ }^{60} \mathrm{Co} \text { Corradiations } \\
\left(4.2 \times 10^{5}\right)\end{array}$ & $"$ & $1.70 \times 10^{8}$ & -11.2 & - & " & $"$ \\
\hline Amber11te IRC-50 & - & 10.7 & $\begin{array}{l}{ }^{182} \text { Ta } \\
(4 \text { to } \\
\left(4 \text { to } 4.2 \times 10^{5}\right)\end{array}$ & Dry & $1.68 \times 10^{6}$ & 2.8 & - & $c, h, 1, k$ & {$[34][35][36]$} \\
\hline$"$ & - & 10.7 & " & $"$ & $1.72 \times 10^{7}$ & 1.7 & - & $"$ & $"$ \\
\hline$"$ & - & 10.7 & $\begin{array}{l}60 \text { co r-radiations } \\
\left(4.2 \times 10^{5}\right)\end{array}$ & $"$ & $1.71 \times 10^{8}$ & 8.9 & - & " & " \\
\hline$"$ & - & 10.7 & $\begin{array}{l}{ }^{182} \mathrm{Ta} r-\mathrm{rad} 1 \mathrm{ations} \\
\left(4 \mathrm{To} 4.2 \times 10^{5}\right)\end{array}$ & Wet (f11tered) & $2.13 \times 10^{6}$ & 4.2 & - & $"$ & $"$ \\
\hline$"$ & - & 10.7 & $"$ & $"$ & $1.73 \times 10^{7}$ & 0.9 & - & " & $"$ \\
\hline$"$ & - & 10.7 & $\left(4.2 \times 10^{5}\right)$ & $"$ & $1.83 \times 10^{8}$ & 5.6 & - & $"$ & $"$ \\
\hline Amberlite $X_{F} \cdots 89$ & - & 12.1 & $\begin{array}{l}{ }^{182} \mathrm{Ta} \text { r-radiations } \\
\left(4.0 \text { to } 4.2 \times 10^{5}\right)\end{array}$ & Dr: & $1.68 \times 10^{6}$ & 0 & - & $c, h, 1, k$ & {$[34][35][36]$} \\
\hline$"$ & - & 17.1 & $"$ & $"$ & $1.72 \times 10^{7}$ & 0.8 & - & $"$ & " \\
\hline$"$ & - & 12.1 & $\begin{array}{l}60 \mathrm{Co} r-\mathrm{rad} \text { rat Ions } \\
\left(4.2 \times 10^{5}\right)\end{array}$ & $"$ & $1.71 \times 10^{8}$ & 0 & - & " & $"$ \\
\hline " & - & 12.1 & 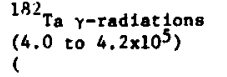 & Wet (fAltered) & $2.13 \times 10^{6}$ & 1.6 & - & $"$ & " \\
\hline " & - & 32.1 & $"$ & $"$ & $1.73 \times 10^{7}$ & 0.8 & - & $"$ & " \\
\hline$"$ & - & 12.1 & " & $"$ & $1.83 \times 10^{8}$ & 2.4 & - & $"$ & $"$ \\
\hline
\end{tabular}




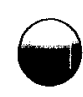

\begin{tabular}{|c|c|c|c|c|c|c|c|c|c|c|}
\hline $\begin{array}{c}\text { RESIN } \\
\text { (Trade Name) } \\
\end{array}$ & IONIC FORM! & $\begin{array}{l}\text { EXCHANCE } \\
\text { CAPACITY } \\
\text { (meg } / 8) \\
\end{array}$ & $\begin{array}{l}\text { RADIATION SOURCE } \\
\text { (Rad/Hr.) }\end{array}$ & $\begin{array}{l}\text { IRRADIATION } \\
\text { CONDITION(S) }\end{array}$ & $\begin{array}{l}\text { INTEGRAL } \\
\text { DOSF } \\
\text { (RAD) } \\
\end{array}$ & $\begin{array}{l}\text { LOSS OF } \\
\text { EXCHANGE } \\
\text { CAPACITY } \\
(\%)\end{array}$ & G VALUE & $\begin{array}{c}\text { OTHER } \\
\text { PARAMETERS } \\
\text { MEASURED } \\
\end{array}$ & $\begin{array}{l}\text { REFERENCE } \\
\text { NUMBER } \\
\text { AND NOTES } \\
\end{array}$ & \\
\hline Amberl1te IRC-50 & $\mathrm{it}^{+}$ & 9.76 & $\begin{array}{l}x-r a y \text { machine } \\
\left(\sim 10^{5}\right)\end{array}$ & Alr-dry & $2.1 \times 10^{8}$ & 39 & - & $a, d, g, 1, k$ & {$[37]$} & \\
\hline$"$ & $\mathrm{H}^{+}$ & 9.76 & $"$ & $"$ & $22 \times 10^{8}$ & 62 & - & $"$ & $"$ & \\
\hline$"$ & $\mathrm{H}^{+}$ & 9.76 & $"$ & $"$ & $24 \times 10^{8}$ & 81 & - & $"$ & $"$ & \\
\hline$"$ & $\mathrm{H}^{+}$ & 9.76 & $"$ & $"$ & $27.6 \times 10^{8}$ & 95 & - & $"$ & $"$ & \\
\hline$"$ & $\mathrm{H}^{+}$ & 9.76 & $"$ & $"$ & $21.5 \times 109$ & 100 & - & $"$ & $"$ & \\
\hline IMAC $C-12 \times 8$ & $\mathrm{H}^{+}$ & 5.0 & $\begin{array}{l}\text { Reactor Irradiation } \\
\left(2.6 \times 10^{6}\right)\end{array}$ & Air-dry & $2.0 \times 10^{9}$ & - & 0.7 & - & [1] Table & 70 \\
\hline IMAC $C-12 \times 2$ & $\mathrm{H}^{+}$ & 5.0 & Reactor $\left(2.6 \times 10^{6}\right)$ & Alr-dry & $2.0 \times 10^{9}$ & - & 1 & - & [1] Table & 70 \\
\hline IMAC $C-12$ & $\mathrm{H}^{+}$ & 5.31 & $\begin{array}{l}\gamma \text {-radiation } \\
\left(1 \times 10^{6}\right)\end{array}$ & $\begin{array}{l}\text { Resin Imversed in } \\
\text { water }\end{array}$ & $2 \times 10^{7}$ & 1.9 & $\begin{array}{l}2.5 \\
\text { (for } \\
\text { highly } \\
\text { acidic } \\
\text { groups) }\end{array}$ & $e_{m}^{e, h, 1, k, 1,}$ & {$[21]$} & \\
\hline$"$ & $"$ & $"$ & $"$ & $"$ & $4 \times 10^{7}$ & 1.9 & - & $"$ & $"$ & \\
\hline$"$ & $"$ & $"$ & $"$ & $"$ & $7 \times 10^{7}$ & 5.6 & - & $"$ & $"$ & \\
\hline$"$ & $"$ & $"$ & $"$ & $"$ & $1 \times 10^{8}$ & 5.5 & - & $"$ & $"$ & \\
\hline$"$ & $"$ & $"$ & $"$ & $"$ & $1.5 \times 10^{8}$ & 6.6 & - & $"$ & $"$ & \\
\hline$"$ & $"$ & $"$ & $"$ & $"$ & $2.0 \times 10^{8}$ & 5.3 & - & $"$ & $"$ & \\
\hline$"$ & $"$ & $"$ & $"$ & $"$ & $3.0 \times 10^{8}$ & 9.6 & - & $"$ & $"$ & \\
\hline$"$ & $"$ & $"$ & $"$ & " & $4.0 \times 10^{8}$ & 12.6 & - & $"$ & " & \\
\hline$"$ & $"$ & $"$ & $"$ & $"$ & $5.0 \times 10^{8}$ & 14.5 & - & $"$ & $"$ & \\
\hline " & $"$ & $"$ & $"$ & $"$ & $7.0 \times 10^{8}$ & 19.2 & - & $"$ & $"$ & \\
\hline$"$ & $"$ & $"$ & $"$ & $"$ & $1.7 \times 10^{9}$ & 35.2 & - & $"$ & $"$ & \\
\hline$"$ & $"$ & $"$ & $"$ & $"$ & $2.0 \times 10^{9}$ & 37.2 & - & $"$ & $"$ & \\
\hline$"$ & $"$ & $"$ & $"$ & $"$ & $3 \times 10^{9}$ & 40.9 & - & $"$ & $"$ & \\
\hline$"$ & $"$ & $"$ & $"$ & $"$ & $5 \times 10^{9}$ & 51.4 & - & $"$ & $"$ & \\
\hline Zeolex SA & $\mathrm{H}^{+}$ & 3.48 & $\begin{array}{l}{ }^{60} \text { Co radiat Ions } \\
\left(0.19 \times 10^{6}\right)\end{array}$ & Alr-dry & $3.2 \times .0^{7}$ & - & 5 & - & [1] Table & 70 \\
\hline Zoelex SA & $\mathrm{H}^{+}$ & 3.48 & $\gamma$-radiation $\left(2 \times 10^{5}\right)$ & Dry resin & $\begin{array}{l}0.02 \text { to } \\
3.5 \times 10^{7}\end{array}$ & 0 to 4.9 & & $1, k$ & [12] & \\
\hline zeorex & $\mathrm{H}^{+}$ & 1.96 & $\begin{array}{l}{ }^{60} \mathrm{Co} \text { rad1ations } \\
\left(1.87 \times 10^{5}\right)\end{array}$ & A1x-dry & $3.2 \times 10^{7}$ & - & 1.5 & - & [1] Table & 70 \\
\hline Zeorex & $\mathrm{H}^{+}$ & 1.96 & r-radiation $\left(2 \times 10^{5}\right)$ & Dry rea1n & $\begin{array}{l}0.02 \mathrm{tg} \\
3.5 \times 10^{9}\end{array}$ & -5.1 to 2.5 & & $1, k$ & {$[12]$} & \\
\hline Dienon SK-1 & $\mathrm{H}^{+}$ & 3.52 & $\begin{array}{l}{ }^{60} \text { Co radiations } \\
\left(0.19 \times 10^{6}\right)\end{array}$ & Afr-dry & $3.2 \times 10^{7}$ & - & 9 & - & [1] Table & 70 \\
\hline Diaion SK/1 & $\mathrm{H}^{+}$ & 3.52 & y-radiation $\left(2 \times 10^{5}\right)$ & Dry reain & $\begin{array}{l}0.02 \text { tg } \\
3.5 \times 10^{9}\end{array}$ & -2.2 to 9.1 & & $1, k$ & [12] & \\
\hline Nalc1te HCRxB & $\mathrm{H}^{+}$ & 5.0 & $\begin{array}{l}x-\text { ray un } 1 t \\
\left(1.12 \times 10^{8}\right)\end{array}$ & Aft-dry & $2.2 \times 10^{9}$ & - & 1 & - & [1] Table & 70 \\
\hline Nalcite HCR & $\mathrm{H}^{+} / \mathrm{Na}^{+}$ & 4.81 & $x$-ray machine $\left(210^{6}\right)$ & A1r-dry & $21 \times 10^{9}$ & 8.3 & - & $a, d, 8,1, k$ & [37] & \\
\hline Nalcite HCRx1 & $\mathrm{H}^{+} / \mathrm{Na}^{+}$ & 5.53 & $x$-ray machine $\left(\sim 10^{6}\right)$ & Adr-dry & $25 \times 10^{8}$ & 12.4 & - & $a, d, 8,1, k$ & {$[37]$} & \\
\hline Hale1te HCRx2 & $"$ & 5.20 & $"$ & $n$ & $n$ & 11.2 & - & $"$ & $"$ & \\
\hline Nalcite HCRx4 & $"$ & 4.90 & $"$ & $"$ & $"$ & 7.1 & - & $"$ & $"$ & \\
\hline Nale1te GCRxB & $"$ & 4.63 & $"$ & $"$ & $"$ & 2.8 & - & $"$ & $"$ & \\
\hline Nalc1te HCRx12 & $"$ & 4.40 & $"$ & $"$ & $"$ & 2.8 & - & $"$ & $"$ & \\
\hline Nalcite HCRx16 & $"$ & 4.20 & $"$ & $"$ & $"$ & 4.1 & - & $"$ & $"$ & \\
\hline
\end{tabular}


TABLE III (Cont'd)

\begin{tabular}{|c|c|c|c|c|c|c|c|c|c|}
\hline $\begin{array}{c}\text { RESIN } \\
\text { (Trade Name) } \\
\end{array}$ & IONIC PORM & $\begin{array}{l}\text { EXCHANGP, } \\
\text { CAPACITY } \\
\text { (meq/s) } \\
\end{array}$ & $\begin{array}{c}\text { RADIATION SOURCE } \\
\left(\operatorname{Rad} / \mathrm{Hr}_{2}\right) \\
\end{array}$ & $\begin{array}{l}\text { IRRADIATION } \\
\text { CONDITION(S) }\end{array}$ & $\begin{array}{c}\text { INTEGRAL } \\
\text { DOSE } \\
\text { (RAD) } \\
\end{array}$ & $\begin{array}{l}\text { LOSS OF } \\
\text { FXCHANGE } \\
\text { CAPACITY } \\
(\%)\end{array}$ & G VALUE & $\begin{array}{c}\text { OTHER } \\
\text { PARAMETRES } \\
\text { MEASURED } \\
\end{array}$ & $\begin{array}{c}\text { REFERENCE } \\
\text { NUMBER } \\
\text { AND NOTES } \\
\end{array}$ \\
\hline Permutit 0 & $\mathrm{H}^{+}$ & 5.0 & $x$-ray unit & Alr-dry & $2.2 \times 10^{9}$ & - & 0.03 & - & (1) Table 7 \\
\hline Permutit 0 & $\mathrm{H}^{+} / \mathrm{Na}^{+}$ & 4.49 & $x$-ray machine $\left(\sim 10^{6}\right)$ & Air-dry & $21 \times 10^{9}$ & 2.0 & - & $a, d, 8,1, k$ & [37] \\
\hline Permutit $\mathrm{H}-70$ & $\mathrm{H}^{+}$ & 10.0 & $\begin{array}{l}x-r a y \text { un } 1 \mathrm{t} \\
\left(1.12 \times 10^{8}\right)\end{array}$ & Alr-dry & $2.2 \times 10^{9}$ & - & 2.7 & - & [1] Table 7 \\
\hline Permut 1t $\mathrm{H}-70$ & $\mathrm{H}^{+}$ & 10.05 & $x$-ray machine $\left(110^{6}\right)$ & A1r-dry & $22 \times 10^{8}$ & 54 & - & $a, d, g, 1, k$ & [37] \\
\hline Permut 1t H-70 & $\mathrm{H}^{+}$ & 10.05 & $"$ & $"$ & $27.6 \times 10^{8}$ & 95 & - & $"$ & $"$ \\
\hline Catex $S$ & $\mathrm{H}^{+}$ & 3.7 & $\begin{array}{l}{ }^{60} \text { Co rediations } \\
(4.6 \text { to } 16.2) \times 10^{5}\end{array}$ & In water & $2.10^{8}$ & - & 4.3 & - & (1) Table? \\
\hline $\mathrm{Ku}-\mathbf{2}$ & $\mathrm{H}^{+}$ & 5.2 & ${ }^{60}$ Co radiationa & Vacuum & $3 \times 10^{8}$ & - & 0.3 & - & [1] Table 7 \\
\hline $\mathrm{Ku}-\mathbf{2}$ & $\mathrm{H}^{+}$ & 5.2 & $"$ & A1r-dry & $1 \times 10^{8}$ & - & 1.9 & - & $"$ \\
\hline $\mathrm{Ku}-\mathbf{2}$ & $\mathrm{H}^{+}$ & 5.0 & Electron Accelerator & Vacuum & $3 \times 10^{8}$ & - & 0.06 & - & [1] Table 7 \\
\hline $\mathrm{Ku}_{u-2}$ & $\mathrm{H}^{+}$ & 5.0 & $"$ & $"$ & $3 \times 10^{8}$ & - & 0.5 & - & $"$ \\
\hline $\mathrm{xu}-2$ & $\mathrm{H}^{+}$ & 5.0 & $"$ & A1r-dry & $2 \times 10^{9}$ & - & 0.04 & - & $"$ \\
\hline$x_{u+2}$ & $\mathrm{H}^{+}$ & 5.0 & Resctor & Alr-dry & $10^{11}$ & - & 0.006 & - & $"$ \\
\hline $\mathrm{Ku}-2$ & $\mathrm{H}^{+}$ & 5.0 & Reactor & $A i r-d r y$ & $10^{11}$ & - & 0.005 & - & $"$ \\
\hline$K L-2 \times B$ & $\mathrm{H}^{+}$ & 5.0 & ${ }^{60}$ Co rad1at1ons & $\begin{array}{l}\text { Absolutely dry } \\
(2.63 \text { to } 6.55) \times 10^{5}\end{array}$ & $10^{8}$ & - & 0.5 & - & [1] Table 7 \\
\hline$K u-2 \times 8$ & $\mathrm{H}^{+}$ & 4.9 & $\begin{array}{l}\text { Electron Accelerator } \\
\left(6.34 \times 10^{8}\right)\end{array}$ & $A d r=d r y$ & $5 \times 10^{9}$ & - & 1.0 & - & [1] Table 7 \\
\hline $\mathrm{Ku}-2 \times 8$ & $\mathrm{H}^{+}$ & 4.8 & $\begin{array}{l}\text { Electron Accelerator } \\
\left(1.27 \times 10^{9}\right)\end{array}$ & Alr $=d r y$ & $11 \times 10^{10}$ & - & 1.0 & - & $"$ \\
\hline $\mathrm{ku}-2 \times 2$ & $\mathrm{H}^{+}$ & 5.0 & $\begin{array}{l}\text { Electron Accelerator } \\
\left(4.9 \times 10^{8}\right)\end{array}$ & In distilled water & $3.4 \times 10^{9}$ & - & 3.5 & - & [1] Table 7 \\
\hline$x u-2 \times 12$ & $\mathrm{H}^{+}$ & 4.75 & $"$ & $"$ & $3.4 \times 10^{9}$ & - & 1.8 & - & $"$ \\
\hline $\mathrm{Ku}-2$ & $\begin{array}{l}\mathrm{Cr}_{2+}^{3+}, \mathrm{Pe}_{2+}^{3+}, \\
\mathrm{Cu}^{2+}, \mathrm{NO}_{2}^{+\infty}\end{array}$ & 5.0 & $"$ & $"$ & $1.1 \times 10^{9}$ & - & 0.75 & - & $"$ \\
\hline $\mathrm{Ku}-2$ & $\mathrm{H}^{+}$ & 5.0 & $"$ & $"$ & $1.1 \times 10^{9}$ & - & 1.7 & - & $"$ \\
\hline $\mathbf{K u - 2}$ & $\mathrm{Ce}_{2}^{3+}, \mathrm{N1}^{2+}$, & 5.0 & $"$ & $"$ & $1.1 \times 10^{9}$ & - & 2.3 & - & " \\
\hline $\mathrm{Ku}-2$ & $\begin{array}{l}\mathrm{Na}^{+}, \mathrm{Co}^{2+} \\
\mathrm{Ca}^{2+}+\mathrm{La}^{3+}, \\
\mathrm{Th}^{4+},\end{array}$ & 5.0 & $\begin{array}{l}\text { Electron Accelerator } \\
\left(4.9 \times 10^{B}\right)^{\text {A }}\end{array}$ & In dist1lled water & $1.1 \times 10^{9}$ & - & 1.6 & k & [1] Table 7 \\
\hline $\mathrm{Ku}-\mathbf{2}$ & $\mathrm{NH}_{4}^{+}$ & 5.0 & $"$ & $"$ & $1.1 \times 10^{9}$ & - & 1.5 & k & $"$ \\
\hline $\mathrm{Ru}_{u}-2$ & $\mathrm{Al}^{3+}$ & 5.0 & . & $"$ & $1.1 \times 10^{9}$ & - & 1.8 & k & $"$ \\
\hline$k u-2 \times 2$ & $\mathrm{R}^{+}$ & 3.38 & $\begin{array}{l}{ }^{60} \mathrm{Co} \text { radiations } \\
(2.63 \text { to } 6.55) \times 10^{5}\end{array}$ & In water & $5.5 \times 10^{8}$ & - & 2.5 & - & (1), Table ? \\
\hline $\mathrm{Ku}-2 \times 8$ & $\mathrm{H}^{+}$ & 3.38 & $"$ & $"$ & $5.5 \times 10^{8}$ & - & 1.6 & - & $"$ \\
\hline $\mathrm{Ku}-2 \times 24$ & $\mathrm{~B}^{+}$ & 3.38 & $"$ & $"$ & $5.5 \times 10^{8}$ & - & 1.4 & - & $"$ \\
\hline $\mathrm{Ku}-2 \times 8$ & $\mathrm{CH}_{3}^{+}, \mathrm{NH}_{4}^{+}$, & $\begin{array}{c}3.38 \\
1\end{array}$ & $\begin{array}{l}{ }^{60} \text { Co radiations } \\
(2.63 \text { to } 6.55) \times 10^{5}\end{array}$ & In water & $9.3 \times 10^{7}$ & - & 1.8 & k & [1] Table 7 \\
\hline$K u-2 \times 8$ & $\mathrm{k}^{+}, \mathrm{Rb}^{+}$ & 3.38 & $"$ & $"$ & $9.3 \times 10^{7}$ & - & 1.7 & $k$ & $"$ \\
\hline$K u-2 \times 8$ & $\mathrm{Na}^{+}$ & 3.38 & $"$ & $"$ & $9.3 \times 10^{7}$ & - & 1.4 & k & $"$ \\
\hline$K u-2 \times 8$ & $\mathrm{Mg}^{2+}$ & 3.38 & $"$ & $"$ & $2.7 \times 10^{8}$ & - & 1.7 & k & $"$ \\
\hline $\mathrm{Ku}-2 \times 8$ & $\mathrm{Ca}^{2+}, \mathrm{sr}^{2+}$ & 3.38 & $"$ & $"$ & $2.7 \times 10^{8}$ & - & 1.5 & $k$ & $"$ \\
\hline$R_{u-2 \times 8}$ & $\mathrm{Ba}^{2+}$ & 3.38 & $"$ & $"$ & $2.7 \times 10^{8}$ & - & 1.5 & k & $"$ \\
\hline$x u-2 \times 8$ & $\mathrm{Co}^{2+}$ & 3.38 & $"$ & $"$ & $3.8 \times 10^{8}$ & - & 1.2 & k & $"$ \\
\hline $\mathrm{Ku}-2 \times 8$ & $\mathrm{Cu}^{2+}$ & 3.38 & $"$ & $"$ & $3.8 \times 10^{8}$ & - & 0.2 & $\mathbf{k}$ & $"$ \\
\hline
\end{tabular}


TABLE III (Cont'd)

\begin{tabular}{|c|c|c|c|c|c|c|c|c|c|}
\hline $\begin{array}{c}\text { RESIN } \\
\text { (Trade Name) } \\
\end{array}$ & IONIC FORM & $\begin{array}{l}\text { EXCHANGE } \\
\text { CAPACITY } \\
\text { (meq/B) } \\
\end{array}$ & $\begin{array}{c}\text { RADIATION SOURCE } \\
\text { (Rad/Hr.) }\end{array}$ & $\begin{array}{r}\text { IRRADIATION } \\
\text { CONDITION(S) } \\
\end{array}$ & $\begin{array}{c}\text { INTECRAL } \\
\text { DOSF } \\
\text { (RAD) } \\
\end{array}$ & $\begin{array}{l}\text { LOSS OF } \\
\text { EXCFANGE } \\
\text { CAPACITY } \\
(x) \\
\end{array}$ & G VALUE & $\begin{array}{l}\text { OTHER } \\
\text { PARAMETERS } \\
\text { MEASURED } \\
\end{array}$ & $\begin{array}{c}\text { REFERENCE } \\
\text { NUMBER } \\
\text { AND NOTES } \\
\end{array}$ \\
\hline $\mathrm{Ku}-2 \times 8$ & $\mathrm{Fe}^{3+}(n 100 \pi)$ & 3.38 & $\begin{array}{l}{ }^{60} \text { Co rad1ations } \\
(2.63 \text { to } 6.55) \times 10^{5}\end{array}$ & In water & $3.8 \times 10^{8}$ & - & 0.05 & k & [1] Table 77 \\
\hline Ku $-2 \times 8$ & $\mathrm{Fe}^{3+}(220 z)$ & 3.38 & $"$ & $"$ & $3.8 \times 10^{8}$ & - & 0.63 & k & " \\
\hline $\mathrm{Ku}-2 \times 8$ & $\mathrm{Fe}^{3+}(\sim 2 \mathrm{x})$ & 3.38 & $"$ & $"$ & $3.8 \times 10^{8}$ & - & 2.8 & k & $"$ \\
\hline $\mathrm{Ku}-2 \times 8$ & $\mathrm{H}^{+}$ & 5.0 & $"$ & Swollen in water & $3.0 \times 10^{8}$ & - & 2.8 & k & [1] Table 71 \\
\hline Ku-2x8 & $\mathrm{H}^{+}$ & 5.0 & $"$ & Swollen (60\% water) & $10^{8}$ & - & 1.7 & k & $"$ \\
\hline Ku-2×8 & $\mathrm{H}^{+}$ & 5.0 & $"$ & In vater & $3 \times 10^{8}$ & - & 2.1 & k & [1] Table 77 \\
\hline $\mathrm{Ku}-2 \times 2$ & $\mathrm{H}^{+}$ & 5.0 & $"$ & $"$ & $1.5 \times 10^{8}$ & - & 3.0 & - & $"$ \\
\hline$K u-2 \times 24$ & $\mathrm{H}^{+}$ & 5.0 & $"$ & $"$ & $1.5 \times 10^{8}$ & - & 1.7 & - & " \\
\hline $\mathrm{Ku} u-2 \times 24$ & $\mathrm{H}^{+}$ & 5.0 & $"$ & $"$ & $4.2 \times 10^{7}$ & - & 1.4 & - & [1] Table 77 \\
\hline $\mathrm{Ku}-2 \times 8$ & $\mathrm{H}^{+}$ & 5.0 & $"$ & $"$ & $1.2 \times 10^{8}$ & - & 3.8 & - & $"$ \\
\hline$K y-2 \times 8$ & $\mathrm{H}^{+}$ & 4.9 & $\begin{array}{l}\text { Electron accelerstor } \\
\left(5.76 \times 10^{8}\right)\end{array}$ & $\begin{array}{l}\text { In water containing } \\
0.25 \mathrm{MH}{ }_{3} \cdot \mathrm{CHOH} \text {. COMH, } \\
0.5 \mathrm{M} \mathrm{HNO}{ }^{3} \text { and } 0.5 \mathrm{M} \\
\mathrm{CH}_{3} \cdot \mathrm{COOH}^{3}\end{array}$ & $2.3 \times 10^{9}$ & - & 2.0 & k & [1] Table 77 \\
\hline $\mathrm{Ku}-2 \times 8$ & $\mathrm{H}^{+}$ & - & $\begin{array}{l}{ }^{60} \text { Co radiat iona } \\
(2.63 \text { to } 6.55) \times 10^{5}\end{array}$ & In water & $3 \times 10^{8}$ & - & 0.5 & - & [1] Table 78 \\
\hline $\begin{array}{l}\mathrm{Ku}-2 \times 2(4,8,12, \\
16)\end{array}$ & $\mathrm{H}^{+}$ & - & $\begin{array}{l}\text { Electron accelerator } \\
\left(5.04 \times 10^{8}\right)\end{array}$ & In diatilled water & $1.8 \times 10^{9}$ & - & 0.7 & - & [1] Table 78 \\
\hline $\mathrm{Ku}-2$ & $\frac{\mathrm{vo}^{2+}, \mathrm{cu}^{2+}}{\mathrm{cr}^{+}, \mathrm{Fe}^{++}}$ & - & $"$ & $"$ & $10^{9}$ & - & 0.9 & - & " \\
\hline $\mathrm{Ku}-2$ & $\begin{array}{l}\mathrm{un}_{3+}^{3+}, \mathrm{Cu}_{3+}^{2+}, \\
\mathrm{Ce}_{3+,}^{3+} \mathrm{Cr}_{4+}^{+\infty} \\
\mathrm{Fe}^{3+}, \mathrm{Th}^{+}\end{array}$ & - & $"$ & $"$ & $1.1 \times 10^{9}$ & - & 0.4 & $\mathrm{k}$ & $"$ \\
\hline Ku $=2$ & 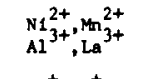 & - & $"$ & $"$ & $1.1 \times 10^{9}$ & - & 0.3 & k & $"$ \\
\hline $\mathrm{Ku}-2$ & $\mathrm{Na}^{+}, \mathrm{NH}_{4}^{+}$ & - & $"$ & $"$ & $1.1 \times 10^{9}$ & - & 0.2 & k & $"$ \\
\hline $\mathrm{K}_{\mathrm{u}}-2$ & $H^{+}$ & - & $"$ & In $0.54 \mathrm{~N} \mathrm{HNO}_{3}$ & $1.8 \times 10^{9}$ & - & 1.3 & $\mathrm{k}$ & $"$ \\
\hline Ku-2 & $\mathrm{H}^{+}$ & - & $"$ & In $0.5 \mathrm{~N} \mathrm{CH} \mathrm{CHOH}_{3}$ & $2.3 \times 10^{9}$ & - & 0.6 & - & $"$ \\
\hline $\mathrm{Ku}-2 \times 8$ & $\mathrm{H}^{+}, \mathrm{zn}^{2+}$ & 2.7 & $\begin{array}{l}\text { B, } \gamma \text {-radiations (Ad- } \\
\text { sorbed } 144 \mathrm{Ce},{ }_{147} \mathrm{Pm} \\
\text { and/or } 22 \mathrm{Na} \text { ) }\end{array}$ & $\begin{array}{l}\text { Processing, d1splace- } \\
\text { ment chromatography }\end{array}$ & $\begin{array}{l}6 \times 10^{8} \\
1 \times 10^{9}\end{array}$ & $\begin{array}{l}26.0 \\
48.0\end{array}$ & - & $\mathrm{a}, \mathrm{b}, \mathrm{k}$ & {$[6]$} \\
\hline $\mathrm{Ku}-2 \times 8$ & $\mathrm{H}^{+}$ & 2.7 & $\begin{array}{l}\text { B, r-radiations (ad- } \\
\text { sorbed rad } 18 \text { quelides } \\
\text { of } 144 \mathrm{Ce}, 154 \mathrm{Eu} \text { and } \\
1555_{\mathrm{Eu}}\end{array}$ & $\begin{array}{l}\text { Processing, d1splace- } \\
\text { ment chromatopraphy }\end{array}$ & $\begin{array}{l}6.0 \times 10^{8} \\
1.0 \times 10^{9}\end{array}$ & $\begin{array}{l}20.0 \\
48.0\end{array}$ & 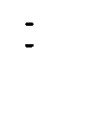 & $b, k$ & [8] \\
\hline $\mathrm{Ku}-2$ & $\mathrm{H}^{+}$ & 4.72 & $\begin{array}{l}{ }^{60} \text { co } \gamma \text {-radiation } \\
\left(4.2 \times 10^{6}\right)\end{array}$ & $\begin{array}{l}\text { In presence of at- } \\
\text { mospheric oxygen }\end{array}$ & $2 \times 10^{8}$ & 3.2 & - & $\begin{array}{l}a, b, c, h, 1 \\
k, 1\end{array}$ & [38] \\
\hline $\mathrm{Ku}-2$ & $\mathrm{H}^{+}$ & 4.72 & $"$ & In vacuum & $2 \times 10^{8}$ & 3.2 & - & $"$ & $"$ \\
\hline Ku-2 & $\mathrm{H}^{+}$ & 4.72 & $"$ & In presence of oxygen & $4 \times 10^{8}$ & 6.1 & - & $"$ & $"$ \\
\hline $\mathrm{xu}-\mathbf{2}$ & $\mathrm{H}^{+}$ & 4.72 & $"$ & In vacuum & $6 \times 10^{8}$ & 21.0 & - & $"$ & " \\
\hline $\mathrm{Ku}-2$ & $\mathrm{Fe}^{3+}$ & 4.72 & $"$ & $"$ & $6 \times 10^{8}$ & 10.8 & - & " & $"$ \\
\hline $\mathrm{ku}-2$ & $\mathrm{Cu}^{2+}$ & 4.72 & $"$ & $"$ & $6 \times 10^{8}$ & 8.7 & - & $"$ & " \\
\hline $\mathrm{Ku}=2$ & $\mathrm{Ce}^{4+}$ & 4.72 & " & " & $6 \times 10^{8}$ & 11.4 & - & $"$ & $"$ \\
\hline $\mathrm{Ku}-2$ & $\mathrm{H}^{+}$ & 4.72 & $"$ & $"$ & $1 \times 10^{9}$ & 18.3 & - & $"$ & $"$ \\
\hline $\mathrm{Ku}_{u}-2$ & $\mathrm{Pe}^{3+}$ & 4.72 & $"$ & $"$ & $1 \times 10^{9}$ & 8.1 & - & $"$ & $"$ \\
\hline $\mathrm{Ku}-2$ & $\mathrm{Cu}^{2+}$ & 4.72 & " & $"$ & $1 \times 10^{9}$ & 9.7 & - & $"$ & $"$ \\
\hline $\mathrm{Ku}_{u-2}$ & $\mathrm{H}^{+}$ & 4.72 & $"$ & In presence of oxygen & $1 \times 10^{9}$ & 16.3 & - & $"$ & $"$ \\
\hline $\mathrm{Ku}-2$ & $\mathrm{Na}^{+}$ & 4.72 & Accelerated electrons & In presence of oxygen & $2.1 \times 10^{9}$ & 0.4 & - & $\begin{array}{l}a, b, c, h, 1 \\
k, 2\end{array}$ & {$[88]$} \\
\hline $\mathrm{Ku}-\mathbf{2}$ & $\mathrm{H}^{+}$ & 4.72 & ${ }^{60}$ Co y-radietion & In vacuum & $2.1 \times 10^{9}$ & 21.0 & - & $\begin{array}{l}a, b, c, h, 1 \\
k, 1\end{array}$ & [38] \\
\hline
\end{tabular}




\begin{tabular}{|c|c|c|c|c|c|c|c|c|c|}
\hline $\begin{array}{c}\text { RESIN } \\
\text { (Trade Name) } \\
\end{array}$ & IONIC FORM & $\begin{array}{l}\text { EXCHANGE } \\
\text { CAPACITY } \\
(\mathrm{meg} / \mathrm{R}) \\
\end{array}$ & $\begin{array}{c}\text { RADIATION SOURCE } \\
(\mathrm{Rad} / \mathrm{Hr} .)\end{array}$ & $\begin{array}{l}\text { IRRADIATION } \\
\text { CONDITION(S) }\end{array}$ & $\begin{array}{c}\text { INTEGRAL } \\
\text { DOSE } \\
\text { (RAD) } \\
\end{array}$ & $\begin{array}{c}\text { LOSS OF } \\
\text { EXCCANGE } \\
\text { CAPACTYY } \\
(\%) \\
\end{array}$ & G VALUE & $\begin{array}{c}\text { OTHER } \\
\text { PARAMETERS } \\
\text { MEASURED } \\
\end{array}$ & $\begin{array}{c}\text { REFERENCE } \\
\text { NUMBER } \\
\text { AND NOTES } \\
\end{array}$ \\
\hline $\mathrm{Ku}-2$ & $\mathrm{Fe}^{3+}$ & 4.72 & " & $"$ & $2.1 \times 10^{9}$ & 6.4 & - & $"$ & $"$ \\
\hline Ku-2 & $\mathrm{Cu}^{2+}$ & 4.72 & $"$ & $"$ & $2.1 \times 10^{9}$ & 8.5 & - & $"$ & " \\
\hline $\mathrm{Ku}-2$ & $\mathrm{Ce}^{4+}$ & 4.72 & $"$ & $"$ & $2.1 \times 10^{9}$ & 17.2 & - & $"$ & $"$ \\
\hline $\mathrm{Ku}-2$ & $\mathrm{H}^{+}$ & 4.72 & $"$ & " & $1 \times 10^{8}$ & 2.4 & - & $"$ & " \\
\hline Ku-2 & $\mathrm{H}^{+}$ & 5.0 & $\begin{array}{l}{ }^{60} \mathrm{Co} \gamma \text {-radiation } \\
\left(1.44 \times 10^{6}\right)\end{array}$ & Immersed in water & $2 \times 10^{8}$ & 11.0 & - & $\begin{array}{l}\mathbf{a}, \mathbf{c}, \mathrm{d}, \mathrm{e}, 1 \\
\mathrm{k}, 1\end{array}$ & {$[40]$} \\
\hline $\mathrm{Ku-2}$ & $\mathrm{H}^{+}$ & 5.0 & $"$ & $"$ & $3 \times 10^{8}$ & 21.7 & - & $"$ & $"$ \\
\hline Ku-2 & $\mathrm{H}^{+}$ & 5.0 & $"$ & $"$ & $5 \times 10^{8}$ & 32.4 & - & $"$ & $"$ \\
\hline $\mathrm{Ku}-2$ & $\mathrm{H}^{+}$ & 5.0 & $"$ & $"$ & $1 \times 10^{9}$ & 44.2 & - & $"$ & $"$ \\
\hline $\mathrm{Ku}-2 \times 8$ & $\mathrm{LI}^{+}$ & 4.71 & ${ }^{60}$ co r-radiation & A1r-dry & $10^{8}$ & 8.9 & - & $b, f, h, k, 1$ & {$[42]$} \\
\hline$K_{U-2 \times 8}$ & $\mathrm{Li}^{+}$ & 4.39 & $"$ & Swollen in water & $10^{8}$ & 4.8 & - & $"$ & $"$ \\
\hline $\mathrm{Ku}-2 \times 8$ & $\mathrm{Na}^{+}$ & 4.39 & " & $A i_{r-d}-y$ & $10^{8}$ & 7.1 & - & $"$ & $"$ \\
\hline$K u-2 \times 8$ & $x^{+}$ & 4.09 & $"$ & Swollen in water & $10^{8}$ & 6.1 & - & " & $"$ \\
\hline $\mathrm{Ku}-2 \times 16$ & $\mathrm{Na}^{+}$ & 4.26 & $"$ & Atr-dry & $10^{8}$ & 9.6 & - & $"$ & $"$ \\
\hline $\mathrm{Ku}-2 \times 16$ & $\mathrm{k}^{+}$ & 3.99 & $"$ & $A i_{r}-d r y$ & $10^{8}$ & 10.5 & - & $"$ & $"$ \\
\hline Ku-2x4 & - & 10.0 & $\begin{array}{l}\gamma \text {-radiations } \\
\left(8.64 \times 10^{6}\right)\end{array}$ & In water & $2.0 \times 10^{8}$ & 30.6 & - & $a, h, 1, k, 1$ & [43] \\
\hline$k u-2 \times 4$ & - & 10.0 & $"$ & $"$ & $4.3 \times 10^{8}$ & 46.0 & - & " & $"$ \\
\hline $\mathrm{Ku}-2 \mathrm{x}^{4}$ & - & 10.0 & $"$ & $"$ & $5.9 \times 10^{8}$ & 53.2 & - & $"$ & $"$ \\
\hline $\mathrm{Ku}-2 \times 4$ & - & 10.0 & $"$ & $"$ & $1.11 \times 10^{9}$ & 64.7 & - & $"$ & $"$ \\
\hline Ku-2x4 & - & 10.0 & $"$ & " & $1.48 \times 10^{9}$ & 77.9 & - & $"$ & $"$ \\
\hline Ku-2 $2 x^{4}$ & - & 10.0 & " & " & $2.91 \times 10^{9}$ & 84.9 & - & " & $"$ \\
\hline Ku-2×4 & - & 10.0 & $"$ & $"$ & $4.02 \times 10^{9}$ & 88.5 & - & $"$ & $"$ \\
\hline$K_{u-2 \times 8}$ & - & 8.0 & $\begin{array}{l}\text { Y-radiation } \\
\left(8.46 \times 10^{6}\right)\end{array}$ & In water & $2.0 \times 10^{8}$ & 22.4 & - & $a, h, 1, k, 1$ & {$[43]$} \\
\hline $\mathrm{Ku}-2 \times 8$ & - & 8.0 & $"$ & " & $4.3 \times 10^{8}$ & 33.6 & - & $"$ & " \\
\hline $\mathrm{Ku}-2 \times 8$ & - & 8.0 & $"$ & $"$ & $5.9 \times 10^{8}$ & 42.6 & - & $"$ & $"$ \\
\hline $\mathrm{Ku}-2 \times 8$ & - & 8.0 & " & $"$ & $1.11 \times 10^{9}$ & 55.9 & - & $"$ & $"$ \\
\hline $\mathrm{Ku}-2 \times 8$ & - & 8.0 & $"$ & $"$ & $1.48 \times 10^{9}$ & 62.2 & - & $"$ & " \\
\hline $\mathrm{Ku}-2 \times 8$ & - & 8.0 & $"$ & $"$ & $2.91 \times 10^{9}$ & 76.2 & - & " & " \\
\hline $\mathrm{Ku}-2 \times 8$ & - & 8.0 & $"$ & $"$ & $4.02 \times 10^{9}$ & 83.1 & - & $"$ & $"$ \\
\hline Ku-2x12 & - & 7.5 & $\begin{array}{l}\text { Y-radiation } \\
\left(8.46 \times 10^{6}\right)\end{array}$ & In water & $2.1 \times 10^{8}$ & 20.9 & - & $\mathrm{a}, \mathrm{h}, 1, \mathrm{k}, 1$ & [43] \\
\hline $\mathrm{Ku}=2 \times 12$ & - & 7.5 & $"$ & $"$ & $4.5 \times 10^{8}$ & 35.7 & - & $"$ & $"$ \\
\hline $\mathrm{Ku}-2 \times 12$ & - & 7.5 & $"$ & $"$ & $6.3 \times 10^{8}$ & 42.1 & - & $"$ & $"$ \\
\hline $\mathrm{Ku}=2 \times 12$ & - & 7.5 & $"$ & $"$ & $1.05 \times 10^{9}$ & 53.6 & - & " & $"$ \\
\hline$K u-2 \times 12$ & - & 7.5 & $"$ & $"$ & $2.48 \times 10^{9}$ & 62.3 & - & " & $"$ \\
\hline $\mathrm{Ku}-2 \times 12$ & - & 7.5 & $"$ & $"$ & $2.91 \times 10^{9}$ & 77.6 & - & $"$ & $"$ \\
\hline $\mathrm{Ku}=2 \times 12$ & - & 7.5 & $"$ & $"$ & $4.02 \times 10^{9}$ & 80.8 & - & $"$ & $"$ \\
\hline $\mathrm{Ku}-2 \times 16$ & - & 7.0 & $\begin{array}{l}\text { r-radiat1on } \\
\left(8.46 \times 10^{6}\right)\end{array}$ & In water & $2.0 \times 10^{8}$ & 18.9 & - & $a, h, 1, k, 1$ & [43] \\
\hline$K u=2 \times 16$ & - & 7.0 & $"$ & $"$ & $4.3 \times 10^{8}$ & 30.8 & - & $"$ & $"$ \\
\hline$K u=2 \times 16$ & - & 7.0 & $"$ & " & $6.3 \times 10^{8}$ & 33.9 & - & $"$ & $"$ \\
\hline $\mathrm{Ku}=2 \times 16$ & - & 7.0 & $"$ & $"$ & $1.11 \times 10^{9}$ & 50.4 & - & $"$ & $"$ \\
\hline $\mathrm{Ku}-2 \times 16$ & - & 7.0 & $"$ & $"$ & $1.48 \times 10^{9}$ & 56.0 & - & $"$ & $"$ \\
\hline Ku-2×16 & - & 7.0 & $"$ & $"$ & $2.91 \times 10^{9}$ & 71.8 & - & $"$ & $"$ \\
\hline Ku-2×16 & - & 7.0 & $"$ & $"$ & $4.02 \times 10^{9}$ & 76.6 & - & $"$ & $"$ \\
\hline
\end{tabular}




\begin{tabular}{|c|c|c|c|c|c|c|c|c|c|}
\hline $\begin{array}{c}\text { RESIN } \\
\text { (Trade Name) } \\
\end{array}$ & $\underline{\text { IONIC FORM }}$ & $\begin{array}{l}\text { EXCHANGE } \\
\text { CAPACITY } \\
\text { (meq } / 8) \\
\end{array}$ & $\begin{array}{l}\text { RADIATION SOURCE } \\
(\operatorname{Rad} / \mathrm{Hr} .)\end{array}$ & $\begin{array}{l}\text { IRRADIATION } \\
\text { CONDITION(S) }\end{array}$ & $\begin{array}{c}\text { INTEGRAL } \\
\text { DOSF. } \\
\text { (RAD) } \\
\end{array}$ & $\begin{array}{c}\text { LOSS OF } \\
\text { EXCHANGE } \\
\text { CAPACITY } \\
(z)\end{array}$ & G VALUE & $\begin{array}{c}\text { OTHER } \\
\text { PARAMETERS } \\
\text { MEASURED } \\
\end{array}$ & $\begin{array}{l}\text { REFERENCE } \\
\text { NUMBER } \\
\text { AND NOTES } \\
\end{array}$ \\
\hline$K u-2 \times 24$ & - & 6.1 & $\begin{array}{l}\text { Y-radiation } \\
\left(8.46 \times 10^{6}\right)\end{array}$ & In water & $2.0 \times 10^{8}$ & 17.7 & - & $a, h, 1, k, 1$ & [43] \\
\hline$K_{U}=2 \times 24$ & - & 6.1 & $"$ & $"$ & $4.3 \times 10^{8}$ & 29.4 & - & $"$ & $"$ \\
\hline $\mathrm{Ku}-2 \times 24$ & - & 6.1 & $"$ & $"$ & $5.9 \times 10^{8}$ & 36.0 & - & " & $"$ \\
\hline$K u-2 \times 24$ & - & 6.1 & $"$ & $"$ & $1.11 \times 10^{9}$ & 51.3 & - & $"$ & $"$ \\
\hline Ku-2×24 & - & 6.1 & $"$ & $"$ & $1.48 \times 10^{9}$ & 55.4 & - & $"$ & $"$ \\
\hline $\mathrm{Ku}-2 \times 24$ & - & 6.1 & $"$ & $"$ & $2.91 \times 10^{9}$ & 71.0 & - & $"$ & $"$ \\
\hline $\mathrm{Ku}-2 \times 24$ & - & 6.1 & $"$ & $"$ & $4.02 \times 10^{9}$ & 76.2 & - & $"$ & $"$ \\
\hline$K u=3 A$ & $\mathrm{H}^{+}$ & 4.93 & $\begin{array}{l}{ }^{60} \mathrm{Co} \mathrm{Y-rad1at1on} \\
\left(1.44 \times 10^{6}\right)\end{array}$ & Inmersed in water & $2 \times 10^{8}$ & 13.0 & 3.08 & $\begin{array}{l}a, c, d, e, 1, \\
k, 1\end{array}$ & {$[40]$} \\
\hline$K u-3 A$ & $\mathrm{H}^{+}$ & 4.93 & $"$ & $"$ & $3 \times 10^{8}$ & 19.5 & $"$ & $"$ & $"$ \\
\hline $\mathbf{K u}-3 \mathbf{A}$ & $\mathrm{H}^{+}$ & 4.93 & $"$ & $"$ & $5 \times 10^{8}$ & 30.0 & $"$ & $"$ & $"$ \\
\hline $\mathrm{Ku}-1$ & $\mathrm{H}^{+}$ & 6.15 & ${ }^{60}$ Co radiation & Vacuur & $3 \times 10^{8}$ & - & 1.8 & k & [1] Table 70 \\
\hline $\mathrm{Ku}-1$ & $\mathrm{H}^{+}$ & 5.6 & Electron Accelerator & Vacuurs & $3 \times 10^{8}$ & - & 0.1 & k & [1] Table 70 \\
\hline $\mathrm{Ku}-1$ & $\mathrm{H}^{+}$ & 5.6 & $"$ & $"$ & $3 \times 10^{8}$ & - & 0.4 & k & $"$ \\
\hline $\mathrm{Ku}-1$ & $\mathrm{H}^{+}$ & 5.6 & Reactor & Air-dry & $10^{11}$ & - & 0.004 & - & $"$ \\
\hline $\mathrm{Ku}-1$ & $\mathrm{H}^{+}$ & 5.6 & $"$ & $"$ & $10^{11}$ & - & 0.01 & - & $"$ \\
\hline $\mathbf{K u}-1$ & $\mathrm{H}^{+}$ & 5.6 & $"$ & $"$ & $10^{11}$ & - & 0.004 & k & $"$ \\
\hline$K u-1$ & $\mathrm{H}^{+}$ & 5.6 & $"$ & $"$ & $10^{11}$ & - & 0.01 & k & $"$ \\
\hline Ku-1 & $\mathrm{H}^{+}$ & 4.5 & $\begin{array}{l}\text { Electron accelerator } \\
\left(4.9 \times 10^{8}\right)\end{array}$ & In distilled water & $1.8 \times 10^{9}$ & - & 0.2 & $x$ & [1] Table 77 \\
\hline $\mathrm{Ku}-1$ & $\mathrm{Cr}^{\mathrm{Na}^{+}}{ }^{+} \mathrm{Fe}^{3+}$ & 4.5 & $"$ & $"$ & $1.8 \times 10^{9}$ & - & 0.16 & k & $"$ \\
\hline Ku-1 & $\mathrm{Cu}^{2+}$ & 4.5 & $"$ & $"$ & $1.8 \times 10^{9}$ & - & 0.1 & k & $"$ \\
\hline Ku-1 & $\mathrm{H}^{+}$ & 3.9 & $"$ & $"$ & $3.4 \times 10^{9}$ & - & 0.9 & k & [1] Table 77 \\
\hline Ku-1 & $\mathrm{Na}^{+}$ & 3.9 & $"$ & $"$ & $1.6 \times 10^{9}$ & - & 0.8 & k & $"$ \\
\hline Ku-1 & $\mathrm{Na}^{+}$ & 3.9 & $"$ & In $0.5 \mathrm{M} \mathrm{CH} \mathrm{CH}_{3} \mathrm{COH}$ & $1.6 \times 10^{9}$ & - & 0.9 & $\mathbf{k}$ & $"$ \\
\hline$K_{u-1}$ & $\mathrm{Na}^{+}$ & - & $\begin{array}{l}\text { Electron accelerator } \\
\left(4,9 \times 10^{8}\right)\end{array}$ & In distilled water & $1.7 \times 10^{9}$ & - & 1.3 & k & [1] Table 78 \\
\hline $\mathrm{Ku}-1$ & $\mathrm{H}^{+}$ & - & $"$ & $0.5 \mathrm{~N} \mathrm{HNO}_{3}$. & $1.7 \times 10^{9}$ & - & 1.2 & k & $"$ \\
\hline $\mathrm{Ku}-1$ & $\mathrm{H}^{+}$ & - & $"$ & In distilled water & $3.4 \times 10^{9}$ & - & 0.2 & - & $"$ \\
\hline $\mathrm{Ku}-32$ & $\mathrm{H}^{+}$ & 3.63 & $\begin{array}{l}\text { r-radiation }\left({ }^{60} \mathrm{Co}\right) \\
\left(1.8 \times 10^{6}\right)\end{array}$ & Molst, Swollen & $2 \times 10^{8}$ & 4.2 & - & $a, b, k$ & [6] \\
\hline$K u=33$ & $\mathrm{H}^{+}$ & 4.01 & $"$ & $"$ & $"$ & 9.2 & - & $"$ & $"$ \\
\hline $\mathrm{Ku}=32$ & $\mathrm{H}^{+}$ & 2.82 & $\begin{array}{l}{ }^{60} \text { Co r-tadiation } \\
\left(1.44 \times 10^{h}\right)\end{array}$ & Inmersed in water & $2 \times 10^{8}$ & 4.2 & 0.58 & $\begin{array}{l}a, c, d, e, f \\
k, 1\end{array}$ & [40] \\
\hline $\mathrm{Ku}-32$ & $\mathrm{H}^{+}$ & 2.82 & $"$ & $"$ & $5 \times 10^{8}$ & 5.6 & $"$ & $"$ & $"$ \\
\hline $\mathrm{Ru}-33$ & $\mathrm{H}^{+}$ & 3.03 & $\begin{array}{l}{ }^{60} \mathrm{Co} \text { r-radiation } \\
\left(1.44 \times 10^{6}\right)\end{array}$ & Imereed in vater & $2 \times 10^{8}$ & 9.2 & 1.35 & $\begin{array}{l}a, c, d, e, 1 \\
k, 1\end{array}$ & [40] \\
\hline $\mathrm{Ku}-33$ & $\mathrm{H}^{+}$ & 3.03 & $"$ & $"$ & $3 \times 10^{8}$ & 9.2 & $"$ & $"$ & $"$ \\
\hline $\mathrm{Ku}-33$ & $\mathrm{H}^{+}$ & 3.03 & $"$ & $"$ & $5 \times 10^{8}$ & 13.0 & $"$ & $"$ & $"$ \\
\hline $\mathrm{Ku}-33$ & - & 2.3 & Accelerated electrons & At $\mathrm{pH} 4.0$ & $9.6: 510^{8}$ & -30 & $\begin{array}{l}\left(\mathrm{SO}_{4}^{2-}\right) \\
0.007- \\
0.09\end{array}$ & $a, b, 1, k, 1$ & $\begin{array}{l}\left.\text { [39] } \mathrm{C}_{\left(\mathrm{SO}_{4}^{2-}\right.}\right) \\
\text { calculated }\end{array}$ \\
\hline $\mathrm{Ku}-33$ & - & 2.3 & $"$ & At $\mathrm{pH} 4.0$ & $1.9 \times 10^{9}$ & -27 & - & $"$ & $"$ \\
\hline $\mathrm{Ku}-33$ & - & 4.65 & $"$ & At $\mathrm{pH} 8.5$ & $9.6 \times 10^{8}$ & -11.0 & - & $"$ & $"$ \\
\hline $\mathrm{Ku}-33$ & - & 4.65 & $"$ & At $\mathrm{pH} 8.5$ & $1.9 \times 10^{9}$ & -19.5 & - & $"$ & $"$ \\
\hline $\mathrm{Ku}-33$ & - & 7.45 & $"$ & At $\mathrm{pH} 11.0$ & $9.6 \times 10^{8}$ & None & - & $"$ & $"$ \\
\hline
\end{tabular}


TABLE III (Cont'd)

\begin{tabular}{|c|c|c|c|c|c|c|c|c|c|}
\hline $\begin{array}{c}\text { RESIN } \\
\text { (Trade Name) } \\
\end{array}$ & IONIC FORM & $\begin{array}{l}\text { EXCHANGE } \\
\text { CAPACITY } \\
\text { (me } / \mathrm{g}) \\
\end{array}$ & $\begin{array}{l}\text { RADIATION SOURCE } \\
(\mathrm{Rad} / \mathrm{Hr} .)\end{array}$ & $\begin{array}{l}\text { IRRADIATION } \\
\text { CONDITION(S) }\end{array}$ & $\begin{array}{c}\text { INTEGRAL } \\
\text { DOSE } \\
\text { (RAD) } \\
\end{array}$ & $\begin{array}{l}\text { LOSS OF } \\
\text { EXCHANGE } \\
\text { CAPACTYY } \\
(\%) \\
\end{array}$ & G VALUE & $\begin{array}{c}\text { OTHER } \\
\text { PARAMETERS } \\
\text { MEASURED } \\
\end{array}$ & $\begin{array}{c}\text { REFERENCE } \\
\text { NUMBER } \\
\text { AND NOTES } \\
\end{array}$ \\
\hline $\mathrm{Ku}-34$ & - & 2.3 & Accelerated electrons & At $\mathrm{pH} 4.0$ & $1.1 \times 10^{9}$ & None & $\begin{array}{l}\left(\mathrm{SO}_{4}^{2-}\right) \\
0.05\end{array}$ & $a, b, 1, k, 1$ & $\begin{array}{l}\text { [39] } \mathrm{G}\left(\mathrm{SO}^{2-} \mathrm{y}^{-}\right) \\
\text {calculated }\end{array}$ \\
\hline Кu- 34 & - & 2.3 & " & At $\mathrm{pH} 4.0$ & $2.1 \times 10^{9}$ & 5.0 & - & $"$ & $"$ \\
\hline $\mathrm{Ku}-34$ & - & 3.74 & $"$ & At $\mathrm{pH} 8.5$ & $1.1 \times 10^{9}$ & -20 & - & $"$ & $"$ \\
\hline $\mathrm{Ku}-34$ & - & 3.74 & $"$ & At $\mathrm{pH} 8.5$ & $2.1 \times 10^{9}$ & -16 & - & " & $"$ \\
\hline $\mathrm{Ku}-34$ & - & 3.74 & $"$ & At $\mathrm{pH} 11.0$ & $1.1 \times 10^{9}$ & -35 & - & $"$ & $"$ \\
\hline $\mathrm{Ku}-35$ & $\mathrm{H}^{+}$ & 3.33 & $\begin{array}{l}{ }^{60} \mathrm{Co} \text { r-radiation } \\
\left(1.44 \times 10^{6}\right)\end{array}$ & Immersed in water & $2 \times 10^{8}$ & 9.3 & 1.50 & $\begin{array}{l}a, c, d, e, i, \\
k, 1\end{array}$ & {$[40]$} \\
\hline $\mathrm{Ku}-35$ & $\mathrm{H}^{+}$ & 3.33 & $"$ & $"$ & $3 \times 10^{8}$ & 7.5 & $"$ & $"$ & " \\
\hline $\mathrm{Ku}-35$ & $\mathrm{H}^{+}$ & 3.33 & $"$ & $"$ & $5 \times 10^{8}$ & 16.5 & $"$ & " & $"$ \\
\hline $\mathrm{Ku}-35$ & - & 2.3 & Accelerated electrons & At $\mathrm{pH} 4.0$ & $8.0 \times 10^{8}$ & -36 & $\begin{array}{l}\left(\mathrm{SO}_{4}^{2-}\right) \\
0.24 \\
\text { to } 0.26\end{array}$ & $a, b, 1, k, 1$ & $\begin{array}{l}\text { [39] } \mathrm{G}\left(\mathrm{SO}_{4}^{2-}\right) \\
\text { calculated }\end{array}$ \\
\hline Ku -35 & - & 2.3 & " & At $\mathrm{pH} 4.0$ & $1.4 \times 10^{9}$ & -36 & - & $"$ & $"$ \\
\hline $\mathrm{Ku}-35$ & - & 3.44 & $"$ & At $\mathrm{pH} 8.5$ & $8.0 \times 10^{8}$ & -57 & - & $"$ & $"$ \\
\hline $\mathrm{Ku}-35$ & - & 3.44 & $"$ & At $\mathrm{pH} 8.5$ & $1.4 \times 10^{9}$ & -90 & - & $"$ & $"$ \\
\hline $\mathrm{Ku}-36$ & $\mathrm{H}^{+}$ & 2.48 & $\begin{array}{l}60 \mathrm{Co} \gamma \text {-radiation } \\
\left(1.44 \times 10^{6}\right)\end{array}$ & Itrnersed in water & $2 \times 10^{8}$ & 0.8 & 0.10 & $\begin{array}{l}a, c, d, e, 1, \\
k, 1\end{array}$ & [40] \\
\hline$k u-36$ & $\mathrm{H}^{+}$ & 2.48 & " & " & $5.36 \times 10^{8}$ & 6.0 & $"$ & $"$ & " \\
\hline Ku-16 & $\mathrm{H}^{+}$ & 2.48 & $"$ & $"$ & $1 \times 10^{9}$ & 10.2 & $"$ & $"$ & $"$ \\
\hline $\mathrm{Ku}-36$ & - & 2.34 & Accelerated electrons & At $\mathrm{pH} 4.0$ & $1.1 \times 10^{9}$ & 2.3 & $\begin{array}{l}\left(\mathrm{So}_{4}^{2-}\right) \\
0.06\end{array}$ & $a, b, 1, k, 1$ & $\begin{array}{l}\text { [39] } \mathrm{G}\left(\mathrm{SO}_{4}^{2-}\right) \\
\text { calculated }\end{array}$ \\
\hline $\mathrm{Ku}-36$ & - & 2.34 & $"$ & At $\mathrm{pH} 4.0$ & $1.9 \times 10^{9}$ & 8.3 & - & $"$ & $"$ \\
\hline $\mathrm{Ku}-36$ & - & 2.34 & $"$ & At $\mathrm{pH} 8.5$ & $1.1 \times 10^{9}$ & 9.0 & - & $"$ & $"$ \\
\hline $\mathrm{Ku}-36$ & - & 2.34 & $"$ & At $\mathrm{pH} 8.5$ & $1.9 \times 10^{9}$ & 8.6 & - & $"$ & $"$ \\
\hline Ku-37 & $\mathrm{H}^{+}$ & 3.20 & $\begin{array}{l}{ }^{60} \text { Co } r \text {-radiations } \\
\left(1.44 \times 10^{6}\right)\end{array}$ & Irunersed in water & $2 \times 10^{8}$ & 10.0 & 1.55 & $\begin{array}{l}a, c, d, e, 1 \\
k, 1\end{array}$ & [40] \\
\hline Ku-37 & $\mathrm{H}^{+}$ & 3.20 & $"$ & $"$ & $3.10^{8}$ & 11.5 & $"$ & " & $"$ \\
\hline $\mathrm{Ku}-37$ & - & 3.0 & Accelerated electrons & AE $\mathrm{pH} 4.0$ & $8.0 \times 10^{8}$ & -4.0 & $\begin{array}{l}\left(\mathrm{so}_{4}^{2-}\right) \\
0.18 \\
\text { to } 0.2\end{array}$ & $a, b, 1, k, 1$ & $\begin{array}{l}\text { [39] } \mathrm{G}\left(\mathrm{SO}_{4}^{2-}\right) \\
\text { calculated }\end{array}$ \\
\hline $\mathrm{Ku}-37$ & - & 3.0 & $"$ & At $\mathrm{pH} 4.0$ & $1.4 \times 10^{9}$ & -4.0 & - & $"$ & $"$ \\
\hline Ku=37 & - & 4.45 & $"$ & At $\mathrm{pH} 8.5$ & $8.0 \times 10^{8}$ & -14 & - & $"$ & $"$ \\
\hline кu-37 & - & 4.45 & $"$ & At $\mathrm{pH} 8.5$ & $1.4 \times 10^{9}$ & -27 & - & $"$ & $"$ \\
\hline Ku-37 & - & 6.2 & $"$ & At $\mathrm{pH} 11.0$ & $8.0 \times 10^{8}$ & -18.4 & - & $"$ & $"$ \\
\hline $\mathrm{Ku}-38$ & $\mathrm{H}^{+}$ & 3.41 & $\begin{array}{l}{ }^{60} \mathrm{Co} \gamma \text {-radiation } \\
\left(1.44 \times 10^{6}\right)\end{array}$ & Immersed in water & $2 \times 10^{8}$ & None & 0.39 & $\begin{array}{l}a, c, d, e, 1 \\
k, 1\end{array}$ & [40] \\
\hline $\mathrm{Ku}-\mathbf{3 8}$ & $\mathrm{H}^{+}$ & 3.41 & $"$ & $"$ & $5.36 \times 10^{8}$ & 9.3 & $"$ & $"$ & $"$ \\
\hline $\mathrm{Ku}-38$ & $\mathrm{H}^{+}$ & 3.41 & $"$ & $"$ & $1 \times 10^{9}$ & 10.6 & $"$ & $"$ & $"$ \\
\hline $\mathrm{Ku}-\mathbf{3 8}$ & - & 3.1 & Acceleraced electron & As $\mathrm{pH} 4.0$ & $9.6 \times 10^{8}$ & $\begin{array}{l}3.5 \\
\text { to } 0.12\end{array}$ & $\begin{array}{l}\left(50_{4}^{2-}\right) \\
0.18 \\
\text { to } 0.12\end{array}$ & $a, b, 1, k, 1$ & lalculated \\
\hline Ku-38 & - & 3.1 & $"$ & At $\mathrm{pH} 4.0$ & $2.1 \times 10^{9}$ & 3.5 & - & $"$ & $"$ \\
\hline $\mathrm{ku}-\mathbf{3 8}$ & - & 5.2 & $"$ & At $\mathrm{pH} 8.5$ & $9.6 \times 10^{8}$ & -25.0 & - & $"$ & $"$ \\
\hline $\mathrm{K}_{u}-\mathbf{3 8}$ & - & 5.2 & $"$ & At $\mathrm{pH} 8.5$ & $2.1 \times 10^{9}$ & -22.5 & - & $"$ & $"$ \\
\hline Ku-38 & - & 7.0 & $"$ & At $\mathrm{pH} 11.0$ & $9.6 \times 10^{9}$ & -20 & - & $"$ & $"$ \\
\hline $\mathrm{ku}=40$ & $\mathrm{H}^{+}$ & 3.25 & $\begin{array}{l}60 \mathrm{Co} \gamma \text {-radiation } \\
\left(1.44 \times 10^{6}\right)\end{array}$ & Inmersed in water & $2 \times 10^{8}$ & 1.8 & 0.29 & $\begin{array}{l}a, c, d, e, 1, \\
k, 1\end{array}$ & {$[40]$} \\
\hline $\mathrm{Ku}=40$ & $\mathrm{H}^{+}$ & 3.25 & $"$ & $"$ & $3 \times 10^{8}$ & 4.8 & $"$ & $"$ & $"$ \\
\hline$K u-40$ & $\mathrm{H}^{+}$ & 3.25 & $"$ & " & $5 \times 10^{8}$ & 5.7 & $"$ & $"$ & $"$ \\
\hline $\mathrm{Ku}-40$ & - & 3.2 & Accelerated electron & At $\mathrm{pH} 4.0$ & $9.6 \times 10^{8}$ & -25.0 & $\begin{array}{l}\left(\mathrm{SO}_{4}^{2-}\right) \\
0.14 \\
\text { so } 0.16\end{array}$ & $a, b, 1, k, 1$ & $\begin{array}{l}\text { [39] } \mathrm{G}\left(\mathrm{SO}_{4}^{2-}\right) \\
\text { calculated }\end{array}$ \\
\hline
\end{tabular}


TABLE III (Cont'd)

\begin{tabular}{|c|c|c|c|c|c|c|c|c|c|}
\hline $\begin{array}{c}\text { RESIN } \\
\text { (Trade Name) } \\
\end{array}$ & IONIC FORM & $\begin{array}{l}\text { FXCHANCE } \\
\text { CAPACITY } \\
(\text { meq } / g) \\
\end{array}$ & $\begin{array}{l}\text { RADIATION SOIRCE } \\
\left(\operatorname{Rad} / \mathrm{Hr}_{.}\right)\end{array}$ & $\begin{array}{l}\text { IRRADIATION } \\
\text { CONDITION(S) }\end{array}$ & $\begin{array}{l}\text { INTEGRAL } \\
\text { DOSE } \\
\text { (RAD) }\end{array}$ & $\begin{array}{l}\text { LOSS OF } \\
\text { EXCHANGE } \\
\text { CAPACITY } \\
(\%)\end{array}$ & G VALUE & $\begin{array}{l}\text { OTHER } \\
\text { PARAMETERS } \\
\text { MEASURED } \\
\end{array}$ & $\begin{array}{l}\text { REFERENCE } \\
\text { NUMBER } \\
\text { AND NOTES }\end{array}$ \\
\hline $\mathrm{Ku}-40$ & - & 3.2 & $"$ & At $\mathrm{pH} 4.0$ & $1.9 \times 10^{9}$ & -22.5 & - & $"$ & $"$ \\
\hline $\mathrm{Ku}-4 n$ & - & 4.1 & $"$ & At $\mathrm{pH} 8.5$ & $9.6 \times 10^{8}$ & -20 & - & $"$ & $"$ \\
\hline$K u-4 \times 30$ & $H^{+}$ & 3.3 & $\begin{array}{l}\text { B, y-radiations }(\mathrm{Ad-}- \\
\text { sorbed } 144 \mathrm{Ce}, 14 f_{\mathrm{Pm}} \\
\text { and/or } 22 \mathrm{Ne})\end{array}$ & $\begin{array}{l}\text { Processing, displace- } \\
\text { ment chromatography }\end{array}$ & $4.5 \times 10^{8}$ & 3.0 & - & $b, k$ & {$[7]$} \\
\hline $\mathrm{Ku}-4 \times 30$ & $\mathrm{H}^{+}$ & 3.8 & $"$ & $"$ & $\begin{array}{l}5.0 \times 10^{8} \\
1.5 \times 10^{9}\end{array}$ & $\begin{array}{l}12.7 \\
25.6\end{array}$ & - & $"$ & $"$ \\
\hline$K u-4 \times 30$ & $\mathrm{H}^{+}$ & 2.8 & $"$ & $"$ & $5.0 \times 10^{8}$ & 3.0 & - & $"$ & $"$ \\
\hline$K u-4 \times 30$ & $\mathrm{H}^{+}$ & 3.0 & $"$ & $"$ & $2.0 \times 10^{9}$ & 21.0 & - & $"$ & $"$ \\
\hline$K u-4 \times 12$ & $\mathrm{H}^{+}$ & 3.9 & Accelerated electron & In wacer & $4.8 \times 10^{8}$ & 15 & - & $a, b, k, l$ & {$[41]$} \\
\hline $\mathrm{Ku}-4 \times 12$ & $\mathrm{H}^{+}$ & 3.8 & $"$ & $"$ & $9.6 \times 10^{8}$ & 17 & - & $"$ & $"$ \\
\hline$K u-4 \times 20$ & $\mathrm{H}^{+}$ & 3.4 & Accelerated electron & In water & $4.8 \times 10^{8}$ & 27 & - & $a, b, k, 1$ & [41] \\
\hline $\mathrm{Ku}=4 \times 20$ & $\mathrm{H}^{+}$ & 3.5 & $"$ & $"$ & $1.9 \times 10^{9}$ & 30 & - & $"$ & $"$ \\
\hline $\mathrm{Ku}-4 \times 30$ & $\mathrm{H}^{+}$ & 4.3 & Accelerated electron & In Water & $4.8 \times 10^{8}$ & 12.7 & - & $a, b, k, 1$ & [41] \\
\hline$K u-4 \times 30$ & $\mathrm{H}^{+}$ & 3.8 & $"$ & $"$ & $1.44 \times 10^{9}$ & 25.6 & - & $"$ & " \\
\hline $\mathrm{Ku}-4 \times 40$ & $\mathrm{H}^{+}$ & 4.2 & Accelerated electron & In water & $3.2 \times 10^{8}$ & 8 & - & $a, b, k, 1$ & [41] \\
\hline Ku $-4 \times 40$ & $\mathrm{H}^{+}$ & 3.9 & $"$ & $"$ & $4.8 \times 10^{8}$ & 14 & - & $"$ & $"$ \\
\hline$K u-4 \times 40$ & $\mathrm{H}^{+}$ & 3.9 & $"$ & $"$ & $9.6 \times 10^{8}$ & 16 & - & $"$ & $"$ \\
\hline $\mathrm{Ku}-4 \times 40$ & $\mathrm{H}^{+}$ & 3.3 & $"$ & $"$ & $1.9 \times 10^{9}$ & 35 & - & $"$ & $"$ \\
\hline $\mathrm{Ku}-4 \times 50$ & $\mathrm{H}^{+}$ & 3.9 & Accelerated electron & In vater & $4.8 \times 10^{8}$ & 8 & - & $a, b, k, 1$ & [41] \\
\hline$K u-4 \times 50$ & $\mathrm{H}^{+}$ & 3.6 & $"$ & $"$ & $9.6 \times 10^{8}$ & 23 & - & $"$ & $"$ \\
\hline$K u-4 \times 50$ & $\mathrm{H}^{+}$ & 3.5 & $"$ & $"$ & $9.6 \times 10^{8}$ & 33 & - & $"$ & $"$ \\
\hline$K u-4 \times 50$ & $\mathrm{H}^{+}$ & 3.3 & $"$ & $"$ & $1.9 \times 10^{9}$ & 42 & - & $"$ & $"$ \\
\hline$K_{u-4 x 50}$ & $\mathrm{H}^{+}$ & 3.0 & $"$ & $"$ & $2.9 \times 10^{9}$ & 42 & - & $"$ & $"$ \\
\hline$K u-4 \times 60$ & $\mathrm{H}^{+}$ & 4.2 & Accelerated electron & In Water & $4.8 \times 10^{8}$ & 7.0 & - & $a, b, k, 1$ & [41] \\
\hline$x u=4 \times 6 n$ & $\mathrm{H}^{+}$ & 3.9 & $"$ & $"$ & $9.6 \times 10^{8}$ & 6.0 & - & $"$ & $"$ \\
\hline$x_{u}=4 \times 60$ & $\mathrm{H}^{+}$ & 3.4 & $"$ & $"$ & $1.9 \times 10^{9}$ & 33.0 & - & $"$ & $"$ \\
\hline Ru-6 & $4^{+}, 7 \mathrm{n}^{2+}$ & 1.9 & $\begin{array}{l}\text { B,y-radiations (Ad- } \\
\text { sorbed radionuclides } \\
\text { of } 144 \mathrm{Ce}, 154_{\mathrm{Eu}} \text { and } \\
155_{\mathrm{Eu}} \text { ) }\end{array}$ & $\begin{array}{l}\text { Procesolng, dioplace- } \\
\text { ment chromatography }\end{array}$ & $\begin{array}{l}6.7 \times 10^{8} \\
2.0 \times 10^{9}\end{array}$ & $\begin{array}{l}0 \\
0\end{array}$ & - & $\stackrel{b, k}{*}$ & $"$ \\
\hline$K u-160$ & $\mathrm{H}^{+}, \mathrm{Zn}^{2+}$ & 2.0 & " & $"$ & $\begin{array}{l}5.8 \times 10^{8} \\
1.7 \times 10^{9}\end{array}$ & $\begin{array}{l}0 \\
6.5\end{array}$ & $\overline{-}$ & $\stackrel{b, k}{\prime \prime}$ & ${ }^{[8]}$ \\
\hline$K u-158$ & - & 3.0 & Accelerated lectron & At $\mathrm{pH} 4.0$ & $9.6 \times 10^{8}$ & 8.0 & $\begin{array}{l}0.12 \\
\text { to } 0.20\end{array}$ & $a, b, 1, k, 1$ & $\begin{array}{l}\text { [39] } \mathrm{C}\left(\mathrm{SO}_{4}^{2-}\right) \\
\text { calculated }\end{array}$ \\
\hline Ku-158 & - & 3.0 & $n$ & At $\mathrm{pH} 4.0$ & $1.6 \times 10^{9}$ & 13.0 & - & $"$ & $"$ \\
\hline$K u-158$ & - & 3.0 & $"$ & At $\mathrm{pH} 4.0$ & $2.6 \times 10^{9}$ & 13.5 & - & $"$ & $"$ \\
\hline Ku-158 & - & 3.9 & Accelerated electron. & At $\mathrm{pH} 8.5$ & $9.6 \times 10^{8}$ & -16.5 & - & $"$ & $"$ \\
\hline$K_{u-158}$ & - & 3.9 & $"$ & At $\mathrm{pH} 8.5$ & $1.6 \times 10^{9}$ & -8.0 & - & $"$ & $"$ \\
\hline$K_{u-158}$ & - & 3.9 & $"$ & At pH 8.5 & $2.6 \times 10^{9}$ & -33.0 & - & $"$ & $"$ \\
\hline$K u-158$ & - & 4.95 & $"$ & At pH 11.0 & $9.6 \times 10^{8}$ & -44.0 & - & $"$ & $"$ \\
\hline$K u-160$ & - & 2.6 & Accelerated electron & At $\mathrm{pH} 4.0$ & $1.1 \times 10^{9}$ & 5.0 & $\begin{array}{l}\left(\mathrm{so}_{4}^{2-j}\right) \\
0.10 \\
\text { to } 0.12\end{array}$ & $a, b, 1, k, 1$ & $\begin{array}{l}{[39] \mathrm{G}\left(\mathrm{SO}_{4}^{2-}\right)} \\
\text { reported }\end{array}$ \\
\hline$R_{u-160}$ & - & 2.6 & $n$ & At $\mathrm{pH} 4.0$ & $1.6 \times 10^{9}$ & 6.5 & - & $"$ & $"$ \\
\hline Ru-160 & - & 2.6 & $"$ & At $\mathrm{pH} 4.0$ & $2.6 \times 10^{9}$ & 13.6 & - & $"$ & $"$ \\
\hline Ru-160 & - & 3.5 & $"$ & At $\mathrm{PH} 8.5$ & $1.1 \times 10^{9}$ & -32.0 & - & $"$ & $"$ \\
\hline
\end{tabular}


TABLE III (Cont'd)

\begin{tabular}{|c|c|c|c|c|c|c|c|c|c|}
\hline $\begin{array}{c}\text { RESIN } \\
\text { (Trade Name) } \\
\end{array}$ & IONIC FORM & $\begin{array}{l}\text { EXCHANGE } \\
\text { CAPACITY } \\
\text { (meq/B) }\end{array}$ & $\begin{array}{c}\text { RADIATION SOURCE } \\
(\operatorname{Rad} / \mathrm{Hr} .)\end{array}$ & $\begin{array}{l}\text { IRRADIATION } \\
\text { CONDITION(s) } \\
\end{array}$ & $\begin{array}{c}\text { INTEGRAL } \\
\text { DOSE } \\
\text { (RAD) } \\
\end{array}$ & $\begin{array}{c}\text { LOSS OF } \\
\text { EXCHANGE } \\
\text { CAPACITY } \\
(\mathbf{z}) \\
\end{array}$ & G VALUE & $\begin{array}{c}\text { OTHER } \\
\text { PARAMETERS } \\
\text { MEASURED } \\
\end{array}$ & $\begin{array}{c}\text { REFERENCE } \\
\text { NUNBER } \\
\text { AND NOTES } \\
\end{array}$ \\
\hline $\mathrm{Ku}-160$ & - & 3.5 & $"$ & At $\mathrm{pH} 8.5$ & $1.6 \times 10^{9}$ & -28.0 & - & $"$ & $"$ \\
\hline $\mathrm{Ku}-160$ & - & 3.5 & $"$ & At $\mathrm{pH} 8.3$ & $2.6 \times 10^{9}$ & -33.0 & - & " & $"$ \\
\hline $\mathrm{Ku}=160$ & - & 5.35 & $"$ & At $\mathrm{pH} 11.0$ & $1.1 \times 10^{9}$ & -29 & - & $"$ & $"$ \\
\hline$K u-160$ & - & 5.35 & $"$ & At $\mathrm{pH} 11.0$ & $1.6 \times 10^{9}$ & -29 & - & $"$ & $"$ \\
\hline$K_{U}-160$ & - & 5.35 & $"$ & At $\mathrm{pH} 11.0$ & $2.6 \times 10^{9}$ & -29 & - & " & $"$ \\
\hline SBS & $\mathrm{r}^{+}$ & 4.0 & Electron Accelerator & Vacuum & $3 \times 10^{8}$ & - & 0.2 & k & [1] Table 70 \\
\hline SBS & $\mathrm{H}^{+}$ & 4.0 & $"$ & $"$ & $3 \times 10^{8}$ & - & 0.5 & - & $"$ \\
\hline sas & $\mathrm{H}^{+}$ & 4.0 & $"$ & $A 1 r-d r y$ & $2 \times 10^{9}$ & - & 0.3 & k & " \\
\hline SBS & $\mathrm{r}^{+}$ & 3.6 & Reactor & Alr-dry & $10^{11}$ & - & 0.004 & k & $"$ \\
\hline SBS & $\mathrm{H}^{+}$ & 3.6 & $"$ & $"$ & $10^{11}$ & - & 0.004 & k & $"$ \\
\hline SBS-2 & $\mathrm{H}^{+}$ & 2.7 & $\begin{array}{l}\text { E1ectron Accelerator } \\
\left(4.9 \times 10^{8}\right)\end{array}$ & In diot111ed water & $3.5 \times 10^{9}$ & - & 0.17 & k & [1] Table 77 \\
\hline SBS-2 & $\mathrm{H}^{+}$ & 2.4 & $"$ & $"$ & $3.5 \times 10^{9}$ & - & 0.06 & $k$ & $"$ \\
\hline sBs-2 & $\mathrm{B}^{+}$ & - & $\begin{array}{l}\text { Electron Accelerator } \\
\left(5.04 \times 10^{8}\right)\end{array}$ & In $0.5 \mathrm{~N}^{\mathrm{CH}_{3}} \mathrm{COOH}$ & $2.2 \times 10^{9}$ & - & 0.6 & k & [1] Table 78 \\
\hline SBS-2 & $\mathrm{H}^{+}$ & - & $"$ & $0.5 \mathrm{~N} \mathrm{CH}_{3}, \mathrm{CHOH}, \mathrm{COOH}$ & $2.2 \times 10^{9}$ & - & 0.7 & k & " \\
\hline SBS-2 & $\mathbf{u}^{+}$ & - & $"$ & In d1st, lliad water & $3.4 \times 10^{9}$ & - & 0.8 & - & $"$ \\
\hline Dowex-30 & $\mathbf{u}^{+}$ & 4.0 & $\begin{array}{l}{ }^{60} \mathrm{Co},{ }^{144} \mathrm{Co}-\mathrm{Pr} \text { rad1- } \\
\text { at1ons (2.52 to } \\
6.01 \times 105)\end{array}$ & $A 1 r-d z y$ & $9 \times 10^{8}$ & - & 0.1 & - & [1] Table 70 \\
\hline Duolite C-3 & $\mathrm{u}^{+}$ & 2.06 & $\begin{array}{l}{ }^{60} \mathrm{co} \text { radiation } \\
\left(1.87 \times 10^{5}\right)\end{array}$ & Alr-dry & $3.2 \times 10^{7}$ & - & 0.3 & - & [1] Table 70 \\
\hline Duol1te $C-10$ & $\mathrm{H}^{+}$ & 6.8 & r-emitter & Dry & $1.5 \times 10^{8}$ & - & 4.0 & $k$ & [1] Table 70 \\
\hline Duolite C-10 & $\mathrm{B}^{+}$ & 6.80 & 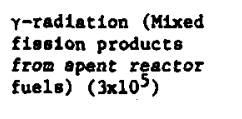 & Dry & $1.5 \times 10^{8}$ & 9.0 & - & $\begin{array}{l}a, b, c, h, 1 \\
k, m\end{array}$ & [22] \\
\hline Duolite cs101 & $\mathbf{u}^{+}$ & 7.85 & $y$-radiation $\left(2 \times 10^{5}\right)$ & Dry. resin & $\begin{array}{c}0.02 \text { to } \\
3.5 \times 10^{7}\end{array}$ & -1.0 to 2.2 & & $1, k$ & [12] \\
\hline$P_{2} \mathbf{s}$ & $\mathbf{B}^{+}$ & 1.82 & $\begin{array}{l}\text { X-ray unit } \\
\left(1.69 \times 10^{5}\right)\end{array}$ & AIr-dry & $1.9 \times 10^{5}$ & - & 520 & k & [1] Table 70 \\
\hline $\mathbf{r}_{2} \mathrm{~s}$ & $\mathrm{~B}^{+}$ & 1.82 & $"$ & Dist1lled water & $1.9 \times 10^{5}$ & - & 150 & $k$ & [1] Table 77 \\
\hline $\mathbf{F}_{4} \mathrm{~S}$ & $\mathrm{~B}^{+}$ & 1.90 & $\begin{array}{l}{ }^{60} \text { Co radiations } \\
\left(1.69 \times 10^{5}\right)\end{array}$ & Air-dry & $1.9 \times 10^{5}$ & - & 780 & k & [1] Table 70 \\
\hline $8_{4} \mathrm{~S}$ & $\mathbf{u}^{+}$ & 1.90 & $"$ & D1st1lled water & $1.9 \times 10^{5}$ & - & 300 & k & [1] Table 7 \\
\hline $\mathbf{P r}_{2} \mathbf{3}$ & $\mathrm{B}^{+}$ & 3.15 & $\begin{array}{l}\text { x-ray unnt } \\
\left(1.69 \times 10^{5}\right)\end{array}$ & Alr-dry & $1.9 \times 10^{5}$ & - & 1300 & k & [1] 70 \\
\hline$r_{2} s$ & $\mathrm{H}^{+}$ & 2.05 & $"$ & $"$ & $1.9 \times 10^{5}$ & - & 360 & k & " \\
\hline $\mathbf{P P}_{2} \mathrm{~s}$ & $\mathrm{H}^{+}$ & 1.90 & " & $"$ & $1.9 \times 10^{5}$ & - & 520 & $\mathbf{k}$ & " \\
\hline$r_{2} s$ & $\mathbf{H}^{+}$ & 3.15 & $"\left(1.2 \times 10^{4}\right)$ & $"$ & $1.9 \times 10^{5}$ & - & 2000 & $k$ & $"$ \\
\hline$r_{2} S$ & $\mathbf{a}^{+}$ & 1.90 & $"\left(1.2 \times 10^{4}\right)$ & $"$ & $1.9 \times 10^{5}$ & - & 830 & k & $"$ \\
\hline $\mathbf{r r}_{2} \mathbf{s}$ & $\mathbf{H}^{+}:$ & 1.90 & $"\left(1.69 \times 10^{5}\right)$ & $"$ & $7.5 \times 10^{5}$ & - & 340 & k & $"$ \\
\hline $\mathbf{P}_{2} \mathbf{S}$ & $\mathrm{B}^{+}, \mathrm{Se}^{+}$ & 1.90 & $"\left(1.2 \times 10^{4}\right)$ & $"$ & $1.9 \times 10^{5}$ & - & 520 & k & $"$ \\
\hline $\mathrm{PP}_{2} \mathrm{~S}$ & $\mathrm{~B}^{+}$ & 1.93 & $\begin{array}{l}60 \text { Co radiations } \\
\left(1.69 \times 10^{5}\right)\end{array}$ & A1r-dry & $1.9 \times 10^{5}$ & $-\cdot$ & 3200 & k & (1) Table 70 \\
\hline $\mathbf{P r}_{2} \mathbf{S}$ & $\mathrm{H}^{+}, \mathrm{me}^{+}$ & 1.93 & $"$ & $"$ & $1.9 \times 10^{5}$ & - & 200 & $k$ & $"$ \\
\hline $\mathbf{m}_{2} \mathrm{~s}$ & $\mathrm{a}^{+}$ & 2.05 & $\begin{array}{l}x-r \text { ay unft } \\
\left(1.69 \times 10^{5}\right)\end{array}$ & Distilled water & $1.9 \times 10^{5}$ & - & 250 & $k$ & [1] Table 77 \\
\hline
\end{tabular}




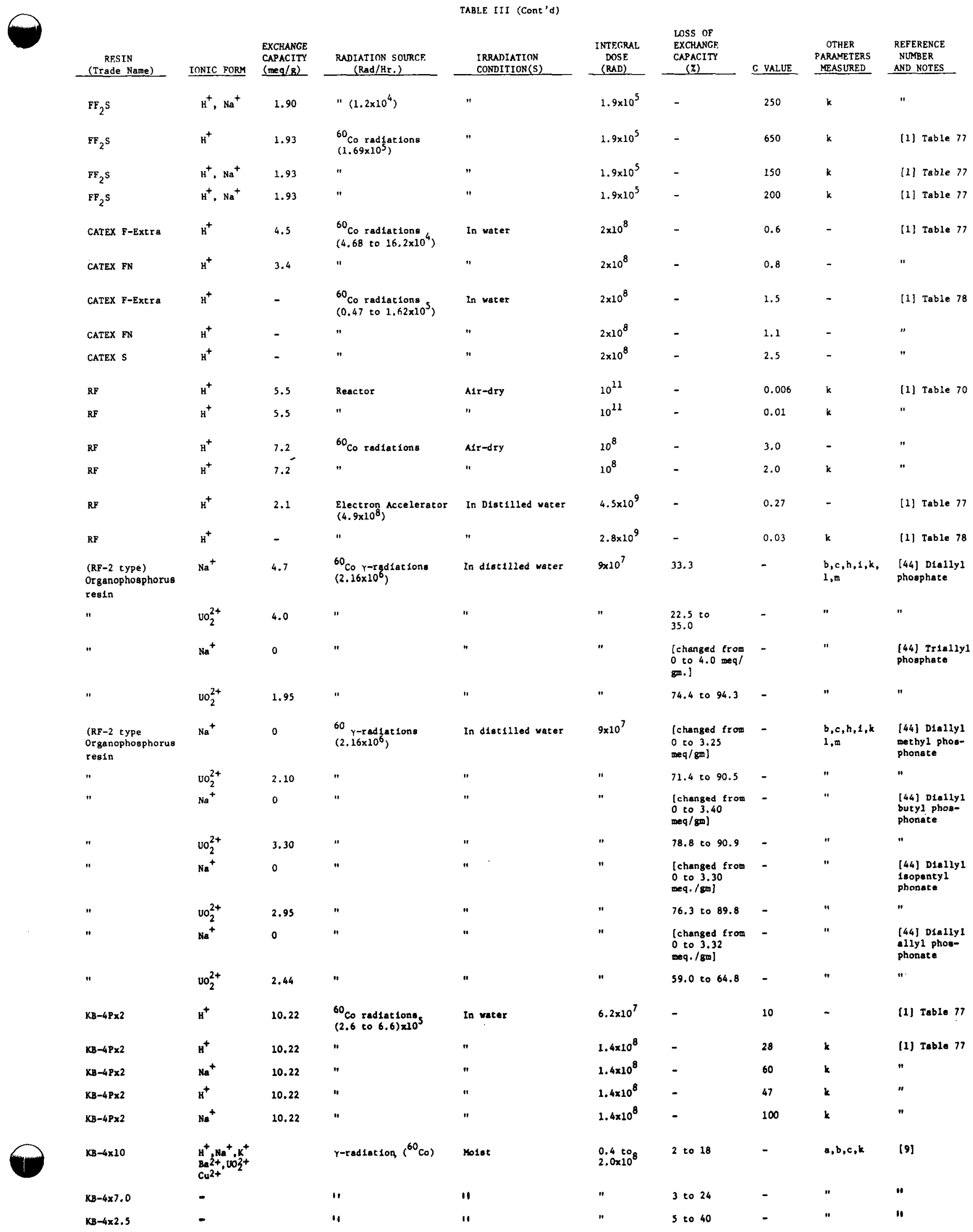


TABLE III (Cont'd)

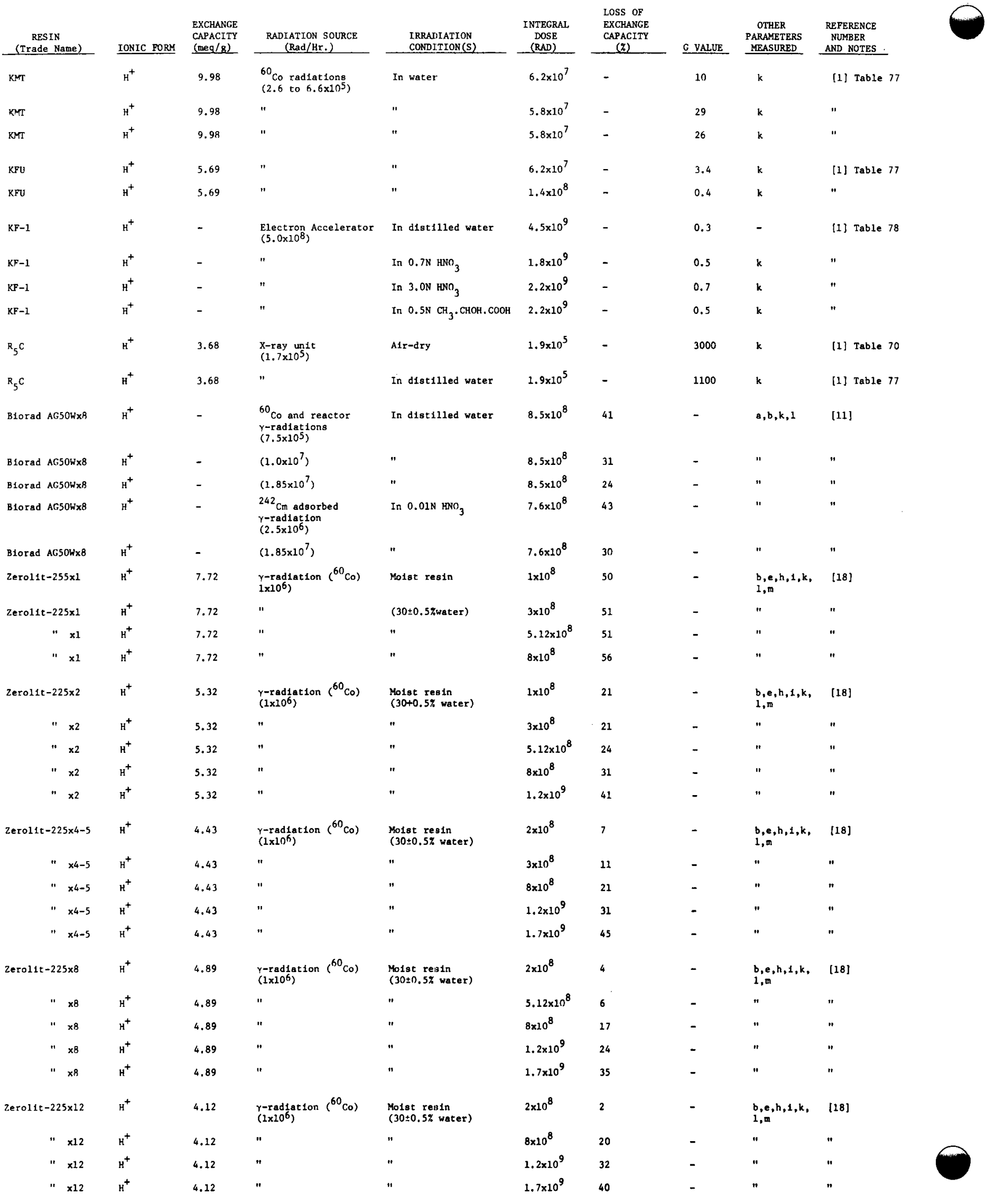


TABLE III (Cont'd)

\begin{tabular}{|c|c|c|c|c|c|c|c|c|c|}
\hline $\begin{array}{c}\text { RESIN } \\
\text { (Trade Name) } \\
\end{array}$ & IONIC FORM & $\begin{array}{l}\text { EXCHANGE } \\
\text { CAPACITY } \\
\text { (meq/8) }\end{array}$ & $\begin{array}{l}\text { RADIATION SOURCE } \\
(\mathrm{Rad} / \mathrm{Hr} .)\end{array}$ & $\begin{array}{l}\text { IRRADLATION } \\
\text { CONDITION (S) }\end{array}$ & $\begin{array}{c}\text { INTEGRAL } \\
\text { DOSE } \\
\text { (RAD) } \\
\end{array}$ & $\begin{array}{l}\text { LOSS OF } \\
\text { EXCHANGE } \\
\text { CAPACITY } \\
(\pi) \\
\end{array}$ & G VALUE & $\begin{array}{c}\text { OTHER } \\
\text { PARAMETERS } \\
\text { MEASURED } \\
\end{array}$ & $\begin{array}{l}\text { REFERENCE } \\
\text { NUMBER } \\
\text { AND NOTES } \\
\end{array}$ \\
\hline Zerollt $-225 \times 20$ & $\mathbf{H}^{+}$ & 3.89 & $\begin{array}{l}\text { r-radiation }\left({ }^{60} \mathrm{Co}\right) \\
\left(1 \times 10^{6}\right)\end{array}$ & $\begin{array}{l}\text { Molst resin } \\
(30 \pm 0.5 z \text { water })\end{array}$ & $2 \times 10^{8}$ & 3 & - & $\begin{array}{l}\mathrm{b}, \mathrm{e}, \mathrm{h}, \mathrm{l}, \mathrm{k}, \\
1, \mathrm{~m}\end{array}$ & {$[18]$} \\
\hline$" . \times 20$ & $\mathrm{H}^{+}$ & 3.89 & $"$ & $"$ & $5.12 \times 10^{8}$ & 7 & - & $"$ & $"$ \\
\hline$" \times 20$ & $\mathrm{H}^{+}$ & 3.89 & $"$ & $"$ & $8 \times 10^{8}$ & 17 & - & $"$ & $"$ \\
\hline$" \times 20$ & $\mathrm{H}^{+}$ & 3.89 & $"$ & $"$ & $1.2 \times 10^{9}$ & 28 & - & $"$ & $"$ \\
\hline $11 \times 20$ & $\mathrm{H}^{+}$ & 3.89 & $"$ & $"$ & $1.7 \times 10^{9}$ & 32 & - & $"$ & $"$ \\
\hline Zeocarb- $225 \times 8$ & $\mathrm{Na}^{+}$ & 4.8 & $\begin{array}{l}\text { Y-radiation } \\
\left(1.63 \times 10^{5}\right)\end{array}$ & $\begin{array}{l}\text { Noist, varyling ratios } \\
\text { of resin to water }\end{array}$ & $1.12 \times 10^{8}$ & 2.33 & - & $b, c, i, j$ & [3] \\
\hline Zeocarb-215 & $\mathrm{H}^{+}$ & 2.6 & $\begin{array}{l}{ }^{60} \mathrm{Co} \gamma-\text { radiations } \\
\left(2.5 \times 10^{5}\right)\end{array}$ & Dry & $\begin{array}{l}(0.5 \text { to } \\
2.0) \times 10^{8}\end{array}$ & - & $\begin{array}{l}\left(\mathrm{H}_{2}\right) \\
0.051\end{array}$ & $c, f, h, k$ & $\begin{array}{l}\text { [47] } \mathrm{G}\left(\mathrm{SO}_{2}\right) \\
\text { is the } \\
\text { inttfal } \mathrm{G} \\
\text { value for the } \\
\text { lowest dose }\end{array}$ \\
\hline$"$ & $"$ & $"$ & $"$ & $" 1$ & $"$ & - & $\begin{array}{l}\left(\mathrm{CO}_{2}\right) \\
0.019\end{array}$ & $"$ & $"$ \\
\hline$"$ & $"$ & $n$ & $"$ & $"$ & $"$ & - & $\begin{array}{l}\left(\mathrm{SO}_{2}\right) \\
0.001\end{array}$ & $"$ & $"$ \\
\hline Zeocarb-215 & $\mathrm{H}^{+}$ & 2.6 & $"$ & $\begin{array}{l}9.2 \text { moles of } \\
\text { water/eq. resin }\end{array}$ & $"$ & - & $\begin{array}{l}\left(\mathrm{H}_{2}\right) \\
0.12\end{array}$ & $"$ & $"$ \\
\hline$"$ & $"$ & $"$ & $"$ & $"$ & $"$ & - & $\begin{array}{l}\left(\mathrm{CO}_{2}\right) \\
0.046\end{array}$ & $"$ & $"$ \\
\hline$"$ & $"$ & $"$ & $"$ & $"$ & $"$ & - & $\begin{array}{l}(\mathrm{cos}) \\
0.005\end{array}$ & $"$ & " \\
\hline$"$ & $"$ & $"$ & $"$ & $"$ & $"$ & - & $\begin{array}{l}\left(\mathrm{SO}_{2}\right) \\
0.005\end{array}$ & $"$ & $"$ \\
\hline Zeocarb-215 & $\mathrm{H}^{+}$ & 2.6 & $"$ & $\begin{array}{l}80 \text { moles of } \\
\text { water/eq. resin }\end{array}$ & $"$ & - & $\begin{array}{l}\left(\mathrm{H}_{2}\right) \\
1.7\end{array}$ & $"$ & "t \\
\hline$"$ & $"$ & $"$ & $"$ & $"$ & $"$ & - & $\begin{array}{l}\left(\mathrm{CO}_{2}\right) \\
0.43\end{array}$ & $"$ & $"$ \\
\hline$"$ & $"$ & 11 & 60 & $"$ & $"$ & - & $\begin{array}{l}\left(\mathrm{SO}_{2}\right) \\
0.002\end{array}$ & $"$ & $"$ \\
\hline $\begin{array}{l}\text { Phosphonic-acid } \\
\text { Resin (DVB/iso- } \\
\text { octane }=10 / 50)\end{array}$ & $\mathrm{Na}^{+}$ & 4.7 & ${ }^{60}$ Co $\gamma$-radiation & In water & $1.9 \times 10^{8}$ & -25 & - & $\begin{array}{l}a, c, f, 1,1, \\
m, n\end{array}$ & [45] \\
\hline$"(10 / 50)$ & $\mathrm{vo}_{2}^{2+}$ & 2.2 & $"$ & $"$ & $"$ & 64 & - & $"$ & $"$ \\
\hline$"(10 / 50)$ & $\mathrm{vo}_{2}^{2+}$ & 2.2 & $"$ & $2 \mathrm{~N} \mathrm{HNO}_{3}$ & $"$ & 92 & - & $"$ & $"$ \\
\hline$"(30 / 50)$ & $\mathrm{Na}^{+}$ & $4.3-4.7$ & $"$ & In water & $"$ & 21 & - & $"$ & $"$ \\
\hline$"(30 / 50)$ & $\begin{array}{l}\mathrm{uo}_{2}^{2+} \\
+\end{array}$ & 2.1 & $"$ & $"$ & $"$ & 12 & - & $"$ & $"$ \\
\hline$"(30 / 50)$ & $\mathrm{Na}^{+}$ & $4.3-4.7$ & $"$ & $2 \mathrm{~N} \mathrm{HNO}_{3}$ & $"$ & -4.5 & - & $"$ & $"$ \\
\hline$"(30 / 50)$ & $\mathrm{yo}_{2}^{2+}$ & 2.1 & $"$ & $n$ & $"$ & 17 & - & $"$ & $"$ \\
\hline$"(40 / 50)$ & $\mathrm{Na}^{+}$ & $3.3-4.7$ & $"$ & In water & $"$ & 12 & - & $"$ & $"$ \\
\hline$"(40 / 50)$ & $\mathrm{UO}_{2}^{2+}$ & 2.0 & $"$ & " & $"$ & 0 & - & $"$ & $"$ \\
\hline$"(40 / 50)$ & $\mathrm{Na}^{+}$ & $3.3-4.7$ & $"$ & $2 \mathrm{~N} \mathrm{HNO}_{3}$ & $"$ & 0 & - & $"$ & " \\
\hline$"(40 / 50)$ & $\mathrm{Uo}_{2}^{2+}$ & 2.0 & $"$ & $"$ & $"$ & 0 & - & $"$ & $"$ \\
\hline $\begin{array}{l}\text { Phosphontc-acid } \\
\text { Resin (DVB/180- } \\
\text { octane }=10 / 70 \text { ) }\end{array}$ & $\mathrm{uO}_{2}^{2+}$ & 5.0 & ${ }^{60} \mathrm{co}$ y-radiacton & In water & $8.7 \times 10^{7}$ & 5 & - & $\begin{array}{l}a, c, f, 1,1 \\
m, n\end{array}$ & [45] \\
\hline$"(10 / 70)$ & $\mathrm{Na}^{+}$ & 8.6 & $"$ & $2 \mathrm{H} \mathrm{HNO}_{3}$ & $9.1 \times 10^{7}$ & 4.6 & - & $"$ & $"$ \\
\hline$"(10 / 100)$ & $\mathrm{Na}^{+}$ & 6.7 & $"$ & In water & $7.6 \times 10^{7}$ & -1.5 & - & $"$ & $"$ \\
\hline$"(10 / 100)$. & $\mathrm{vo}_{2}^{2+}$ & 4.1 & $"$ & $"$ & $"$ & 10 & - & $"$ & " \\
\hline$"(10 / 100)$ & $\mathrm{Na}^{+}$ & 6.7 & $"$ & $2 \mathrm{~N}^{2} \mathrm{wNO}_{3}$ & $8.2 \times 10^{7}$ & -3.0 & - & $"$ & $"$ \\
\hline " $(30 / 100)$ & $\mathrm{Na}^{+}$ & 5.3 & $"$ & In water & $7.6 \times 10^{7}$ & 2.0 & - & $"$ & $"$ \\
\hline$"(30 / 100)$ & $\mathrm{vo}_{2}^{2+}$ & 2.5 & $"$ & $"$ & $"$ & 5.0 & - & $"$ & $"$ \\
\hline$"(30 / 100)$ & $\mathrm{Na}^{+}$ & 5.3 & $"$ & $2 \mathrm{~N} \mathrm{WNO}_{3}$ & $7.8 \times 10^{7}$ & -1.5 & - & $"$ & $"$ \\
\hline$"(30 / 100)$ & $\mathrm{Na}^{+}$ & 5.3 & $"$ & In air & $8.1 \times 10^{7}$ & -4 & - & $"$ & $"$ \\
\hline
\end{tabular}


TABLE III (Cont'd)

\begin{tabular}{|c|c|c|c|c|c|c|c|c|c|}
\hline $\begin{array}{c}\text { RESIN } \\
\text { (Trade Name) } \\
\end{array}$ & IONIC FORM & $\begin{array}{l}\text { EXCHANGE } \\
\text { CAPACITY } \\
\text { (meq/8) } \\
\end{array}$ & $\begin{array}{c}\text { RADIATION SOURCE } \\
(\mathrm{Rad} / \mathrm{Hr} .)\end{array}$ & $\begin{array}{l}\text { IRRADIATION } \\
\text { CONDITION }(\mathrm{S})\end{array}$ & $\begin{array}{c}\text { INTEGRAL } \\
\text { DOSE } \\
\text { (RAD) } \\
\end{array}$ & $\begin{array}{l}\text { LOSS OF } \\
\text { EXCHANGE } \\
\text { CAPACITY } \\
(\%)\end{array}$ & G VALUE & $\begin{array}{c}\text { OTHER } \\
\text { PARAMETERS } \\
\text { MEASURED } \\
\end{array}$ & $\begin{array}{l}\text { REFERENCE } \\
\text { NUMBER } \\
\text { AND NOTES } \\
\end{array}$ \\
\hline$"(12 / 60)$ & $\mathrm{Na}^{+}$ & 8.5 & $"$ & In water & $8.1 \times 10^{7}$ & 7 & - & $"$ & $"$ \\
\hline$"(12 / 60)$ & $\mathrm{vo}_{2}^{2+}$ & 5.5 & $"$ & $"$ & $8.1 \times 10^{7}$ & 7 & - & $"$ & $"$ \\
\hline$"(16 / 60)$ & $\mathrm{Na}^{+}$ & 7.8 & $"$ & $"$ & $7.9 \times 10^{7}$ & 4 & - & $"$ & $"$ \\
\hline$"(16 / 60)$ & $\mathrm{vo}_{2}^{2+}$ & 5.5 & $"$ & $"$ & $"$ & 7 & - & $"$ & $"$ \\
\hline$"(16 / 60)$ & $\mathrm{Na}^{+}$ & 7.8 & $"$ & $2 \mathrm{~N} \mathrm{HNO}_{3}$ & $7.7 \times 10^{7}$ & 3 & - & $"$ & $"$ \\
\hline$"(16 / 60)$ & $\mathrm{UO}_{2}^{2+}$ & 5.5 & 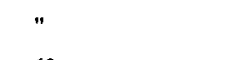 & $"$ & $"$ & 9 & - & $"$ & $"$ \\
\hline $\begin{array}{l}\text { Phosphonic-ac1d } \\
\text { Resin (DVB/180- } \\
\text { octane }=(16 / 60)\end{array}$ & $\mathrm{Na}^{+}$ & 7.8 & ${ }^{60} \mathrm{Co} \gamma$-radiation & In air & $8 \times 10^{7}$ & 4 & - & $\begin{array}{l}a, c, f, f, 1, \\
m, n\end{array}$ & [45] \\
\hline$"(16 / 60)$ & $\mathrm{vo}_{2}^{2+}$ & 5.5 & $"$ & $"$ & $"$ & 10 & - & $"$ & $"$ \\
\hline$"(16 / 80)$ & $\mathrm{Na}^{+}$ & 7.8 & $"$ & In water & $8.1 \times 10^{7}$ & 6 & - & $"$ & $"$ \\
\hline$"(16 / 80)$ & $\mathrm{uO}_{2}^{2+}$ & 5.5 & $"$ & $"$ & $"$ & 6 & - & $"$ & $n$ \\
\hline$"(16 / 80)$ & $\mathrm{Na}^{+}$ & 7.8 & $"$ & $2 \mathrm{~N} \mathrm{KNO}_{3}$ & $7.5 \times 10^{7}$ & 6 & - & $"$ & $"$ \\
\hline$"(16 / 80)$ & $\mathrm{vo}_{2}^{2+}$ & 5.5 & $"$ & $"$ & $"$ & 10 & - & $"$ & $"$ \\
\hline$"(16 / 80)$ & $\mathrm{Na}^{+}$ & 7.8 & $"$ & In air & $7.4 \times 10^{7}$ & 4 & - & $"$ & $"$ \\
\hline$"(16 / 80)$ & $\mathrm{uo}_{2}^{2+}$ & 5.5 & $"$ & $"$ & $"$ & 10 & - & $"$ & $"$ \\
\hline $\begin{array}{l}\text { Sulfonated Poly- } \\
\text { styrene DVB Resin } \\
\text { (8z Commercial } \\
\text { DVB) }\end{array}$ & $\mathrm{H}^{+}$ & 5.29 & $\begin{array}{l}{ }^{60} \mathrm{Co} \gamma \text {-radiation } \\
\left(2.9 \times 10^{5}\right)\end{array}$ & Wet & $9.1 \times 10^{7}$ & 6.6 & - & $a, b, c, k$ & [46] \\
\hline$"$ & $\mathrm{H}^{+}$ & 5.29 & $"$ & $"$ & $1.82 \times 10^{8}$ & 10.8 & - & $"$ & $"$ \\
\hline$"$ & $\mathrm{H}^{+}$ & 5.29 & $"$ & $"$ & $1.90 \times 10^{8}$ & 11.2 & - & $"$ & $"$ \\
\hline$"$ & $\mathrm{H}^{+}$ & 5.29 & $"$ & Dry & $8.8 \times 10^{7}$ & 4.7 & - & $"$ & $"$ \\
\hline$"$ & $\mathrm{H}^{+}$ & 5.29 & $"$ & $"$ & $8.9 \times 10^{7}$ & 4.3 & - & $"$ & $"$ \\
\hline$"$ & $\mathrm{H}^{+}$ & 5.29 & $"$ & $"$ & $1.73 \times 10^{8}$ & 5.5 & - & $"$ & $"$ \\
\hline$"$ & $\mathrm{H}^{+}$ & 5.29 & $"$ & $"$ & $1.75 \times 10^{8}$ & 5.7 & - & $"$ & $"$ \\
\hline (8\% $2: 1 / m: p-D V B)$ & $\mathrm{H}^{+}$ & 5.29 & $"$ & Wet & $9.1 \times 10^{7}$ & 5.8 & - & $"$ & $"$ \\
\hline$"$ & $\mathrm{H}^{+}$ & 5.29 & $"$ & $"$ & $9.2 \times 10^{7}$ & 4.2 & - & $"$ & $"$ \\
\hline$"$ & $\mathrm{H}^{+}$ & 5.29 & $"$ & $"$ & $1.82 \times 10^{8}$ & 9.3 & - & $"$ & $"$ \\
\hline$"$ & $\mathrm{H}^{+}$ & 5.29 & $"$ & $"$ & $1.90 \times 10^{8}$ & 10.2 & - & $"$ & $"$ \\
\hline$"$ & $\mathrm{H}^{+}$ & 5.29 & $"$ & Dry & $8.9 \times 10^{7}$ & 3.2 & - & $"$ & $"$ \\
\hline$"$ & $\mathrm{H}^{+}$ & 5.29 & $"$ & $"$ & $9.0 \times 10^{7}$ & 2.5 & - & $"$ & $"$ \\
\hline$"$ & $\mathrm{H}^{+}$ & 5.29 & $"$ & $"$ & $1.73 \times 10^{8}$ & 5.1 & - & $"$ & $"$ \\
\hline$"$ & $\mathrm{H}^{+}$ & 5.29 & $"$ & $"$ & $1.75 \times 10^{8}$ & 4.5 & - & $"$ & $"$ \\
\hline (8Z p-DVB) & $\mathrm{H}^{+}$ & 5.33 & $"$ & Wet & $9.1 \times 10^{7}$ & 7.1 & - & $"$ & $"$ \\
\hline$"$ & $\mathrm{H}^{+}$ & 5.33 & $"$ & " & $1.14 \times 10^{8}$ & 8.6 & - & $"$ & $"$ \\
\hline$"$ & $\mathrm{H}^{+}$ & 5.33 & $"$ & $"$ & $1.82 \times 10^{8}$ & 10.7 & - & $"$ & $"$ \\
\hline$"$ & $\mathrm{H}^{+}$ & 5.33 & $"$ & Dry & $8.8 \times 10^{7}$ & 3.4 & - & $"$ & $"$ \\
\hline$"$ & $\mathrm{H}^{+}$ & 5.33 & $"$ & $"$ & $9.0 \times 10^{7}$ & 3.4 & - & $"$ & $"$ \\
\hline$"$ & $\mathrm{H}^{+}$ & 5.33 & $"$ & $"$ & $1.73 \times 10^{8}$ & 5.2 & - & $"$ & $"$ \\
\hline$"$ & $\mathrm{H}^{+}$ & 5.33 & $"$ & $"$ & $1.75 \times 10^{8}$ & 4.5 & - & " & $"$ \\
\hline$(8 Z \mathbb{w}-D \sqrt{B})$ & $\mathrm{H}^{+}$ & 5.30 & $"$ & Wet & $9.1 \times 10^{7}$ & 5.8 & - & $"$ & $"$ \\
\hline$"$ & $\mathrm{H}^{+}$ & 5.30 & $"$ & $"$ & $1.14 \times 10^{8}$ & 7.2 & - & $"$ & $"$ \\
\hline $\begin{array}{l}\text { Sulfonated Poly- } \\
\text { atyrene DVB Resin } \\
\text { ( } 8 \pi \text { a-DVB) }\end{array}$ & $\mathrm{H}^{+}$ & 5.30 & $\begin{array}{l}{ }^{60} \mathrm{Co} y-r a d i a t i o n \\
\left(2.9 \times 10^{5}\right)\end{array}$ & Wet & $1.80 \times 10^{8}$ & 10.4 & - & $a, b, c, k$ & {$[46]$} \\
\hline$"$ & $\mathrm{H}^{+}$ & 5.30 & $"$ & Dry & $8.4 \times 10^{7}$ & 3.1 & - & " & $"$ \\
\hline$"$ & $\mathrm{H}^{+}$ & 5.30 & $"$ & $"$ & $1.73 \times 10^{8}$ & 5.3 & - & $"$ & $"$ \\
\hline$"$ & $\mathrm{H}^{+}$ & 5.30 & $"$ & $"$ & $1.75 \times 10^{8}$ & 5.2 & - & $"$ & $"$ \\
\hline
\end{tabular}




\begin{tabular}{|c|c|c|c|c|c|c|c|c|c|}
\hline $\begin{array}{c}\text { RESIN } \\
\text { (Trade Name) } \\
\end{array}$ & IONIC FORM & $\begin{array}{l}\text { EXCHANCE } \\
\text { CAPACITY } \\
\text { (mea } / g) \\
\end{array}$ & $\begin{array}{c}\text { RADIATION SNURCE } \\
\text { (Rad/Hr.) }\end{array}$ & $\begin{array}{l}\text { IRRADIATION } \\
\text { CONDITION(S) }\end{array}$ & $\begin{array}{r}\begin{array}{c}\text { INTFGRAL } \\
\text { DOSF } \\
\text { (RAD) }\end{array} \\
\end{array}$ & $\begin{array}{l}\text { LOSS OF } \\
\text { EXCHANGE } \\
\text { CAPACIYY } \\
(\%) \\
\end{array}$ & C VALUE & $\begin{array}{c}\text { OTHER } \\
\text { PARAMEERES } \\
\text { MEASURED } \\
\end{array}$ & $\begin{array}{c}\text { REFERENCE } \\
\text { NUMBER } \\
\text { AND NOTES } \\
\end{array}$ \\
\hline $\begin{array}{l}\text { Sulfonated Poly- } \\
\text { styrene DVB Resin } \\
\text { (4\% Commercial DVB) }\end{array}$ & $\mathrm{H}^{+}$ & 5.32 & $\begin{array}{l}{ }^{60} \mathrm{Co} \gamma \text {-radiation } \\
\left(2.9 \times 10^{5}\right)\end{array}$ & Wer & $9.1 \times 10^{7}$ & 7.7 & - & $a, b, c, k$ & [46] \\
\hline$"$ & $\mathrm{H}^{+}$ & 5.32 & $"$ & $"$ & $1.82 \times 10^{8}$ & 12.8 & - & $"$ & $"$ \\
\hline$"$ & $\mathrm{H}^{+}$ & 5.32 & $"$ & Dry & $8.5 \times 10^{7}$ & 2.3 & - & $"$ & $"$ \\
\hline$"$ & $\mathrm{H}^{+}$ & 5.32 & $"$ & $"$ & $1.66 \times 10^{8}$ & 5.1 & - & $"$ & $"$ \\
\hline$"$ & $\mathrm{H}^{+}$ & 5.32 & $"$ & $"$ & $1.70 \times 10^{8}$ & 5.1 & - & " & $"$ \\
\hline (4\% $2: 1 / m: p$ DVB $)$ & $\mathrm{H}^{+}$ & 5.34 & $"$ & Het & $9.1 \times 10^{7}$ & 5.6 & - & $"$ & " \\
\hline$"$ & $\mathrm{H}^{+}$ & 5.34 & " & $"$ & $1.76 \times 10^{8}$ & 12.0 & - & $"$ & $"$ \\
\hline$"$ & $\mathrm{H}^{+}$ & 5.34 & $n$ & $"$ & $1.80 \times 10^{8}$ & 12.3 & - & $"$ & $"$ \\
\hline$"$ & $\mathrm{H}^{+}$ & 5.34 & $"$ & Dry & $8.5 \times 10^{7}$ & 2.1 & - & $"$ & $"$ \\
\hline$"$ & $\mathrm{H}^{+}$ & 5.34 & $"$ & $"$ & $1.67 \times 10^{8}$ & 4.8 & - & $"$ & $"$ \\
\hline " & $\mathrm{H}^{+}$ & 5.34 & " & $"$ & $1.70 \times 10^{8}$ & 4.8 & - & $"$ & $"$ \\
\hline (4Z $\mathrm{p}-\mathrm{DVB})$ & $\mathrm{H}^{+}$ & 5.34 & " & wet & $9.1 \times 10^{7}$ & 7.1 & - & " & $"$ \\
\hline$"$ & $\mathrm{H}^{+}$ & 5.34 & $"$ & $"$ & $1.76 \times 10^{8}$ & 12.0 & - & $"$ & " \\
\hline$"$ & $\mathrm{H}^{+}$ & 5.34 & $"$ & Dry & $8.5 \times 10^{7}$ & 2.1 & - & $"$ & " \\
\hline$"$ & $\mathrm{H}^{+}$ & 5.34 & $"$ & $"$ & $1.66 \times 10^{8}$ & 4.5 & - & $"$ & $"$ \\
\hline$"$ & $\mathrm{H}^{+}$ & 5.34 & $"$ & $"$ & $1.70 \times 10^{8}$ & 4.9 & - & " & $"$ \\
\hline ( $4 \% \mathrm{~m}-\mathrm{DVB})$ & $\mathrm{H}^{+}$ & 5.32 & $"$ & Wet & $9.1 \times 10^{7}$ & 6.5 & - & " & $"$ \\
\hline$"$ & $\mathrm{H}^{+}$ & 5.32 & $"$ & $"$ & $1.80 \times 10^{8}$ & 12.0 & - & $"$ & $"$ \\
\hline$"$ & $\mathrm{H}^{+}$ & 5.32 & $"$ & Dry & $8.4 \times 10^{7}$ & 2.6 & - & $"$ & $"$ \\
\hline$"$ & $\mathrm{H}^{+}$ & 5.32 & $"$ & $"$ & $1.66 \times 10^{8}$ & 4.7 & - & $"$ & $"$ \\
\hline
\end{tabular}

(a) weight change, (b) swelling/shrinkage or volume change, (c) vater content, (d) solubility, (e) oxidizability, (f) gas evolution, (g) therwal stability, (h) products of decomposition, (1) formation of ionic species such as $\mathrm{SO}_{4}^{--}, \mathrm{H}^{+}, \mathrm{Cl}^{-}$, etc., (j) use of scavenger, (k) exchange capacity measurements other than TEC, (1) attempt to propose mechanism(s), ( $($ ) study of water soluble products, (n) microscopic examination of resin beads. 
TABLE IV

SUMMARY OF EXPERIMENTAL DATA ON THE EFFECT

OF IONIZING RADIATION ON SYNTHETIC ANION EXCHANGERS

\begin{tabular}{|c|c|c|c|c|c|c|c|c|c|}
\hline $\begin{array}{c}\text { RESIN } \\
\text { (Trade Name) } \\
\end{array}$ & $\underline{\text { IONIC FORM }}$ & $\begin{array}{l}\text { EXCHANGE } \\
\text { CAPACITY } \\
\text { (meg/g) } \\
\end{array}$ & $\begin{array}{c}\text { RADIATION SOURCE } \\
(\mathrm{Rad} / \mathrm{Hr} .)\end{array}$ & $\begin{array}{l}\text { IRRADIATION } \\
\text { CONDITION(S) }\end{array}$ & $\begin{array}{c}\text { INTEGRAL } \\
\text { DOSE } \\
\text { (RAD) } \\
\end{array}$ & $\begin{array}{c}\text { LOSS OF } \\
\text { EXCHANGE } \\
\text { CAPACITY } \\
(\%) \\
\end{array}$ & G VALUE & $\begin{array}{c}\text { OTHER } \\
\text { PARAMETERS } \\
\text { MEASURED } \\
\end{array}$ & $\begin{array}{c}\text { REFERENCE } \\
\text { NUMBER } \\
\text { AND NOTES } \\
\end{array}$ \\
\hline Dowex 1 & $\mathrm{OH}^{-}$ & 3.0 & $\begin{array}{l}{ }^{60} \mathrm{Co} \text { and }{ }^{144} \mathrm{Ce}-\mathrm{Pr} \\
\text { radiations }(2.5 \text { to } \\
\left.6.0 \times 10^{5}\right)\end{array}$ & Alr-dry & $1.0 \times 10^{9}$ & - & 3 & - & [1] Table 70 \\
\hline Dowex $1 \times 8$ & $\mathrm{Cl}^{-}$ & 3.8 & $\gamma$-emitter & Alr-dry & $7.6 \times 10^{7}$ & - & 13 & $\mathbf{k}$ & $"$ \\
\hline Dowex $1 \times 8$ & $\mathrm{OH}^{-}$ & 3.8 & $"$ & Moist & $2.7 \times 10^{9}$ & - & 11 & $\mathbf{k}$ & [1] Table 77 \\
\hline Dowex $1 \times 8$ & $\mathrm{OH}^{-}$ & 3.8 & $"$ & $"$ & $2.7 \times 10^{9}$ & - & 27 & $\mathbf{k}$ & $"$ \\
\hline Dowex $1 \times 8$ & $\mathrm{Cl}^{-}$ & 3.8 & $"$ & Swollen in water & $8.7 \times 10^{7}$ & - & 13 & $\mathbf{k}$ & $"$ \\
\hline Dowex $1 \times 4$ & $\mathrm{NO}_{3}^{-}$ & 4.8 & $"$ & Moist & 1. $1 \times 10^{8}$ & - & 10 & $\mathbf{k}$ & $"$ \\
\hline Dowex $1 \times 4$ & $\mathrm{No}_{3}^{-}$ & 3.8 & ${ }^{60}$ Co radiations & In $7 \mathrm{M} \mathrm{HNO} 3$ & $1.9 \times 10^{8}$ & - & 10 & - & $"$ \\
\hline Dowex $1 \times 8$ & $\mathrm{OH}^{-}$ & - & $\gamma$-emitter & Moist & $2.7 \times 10^{8}$ & - & 3.8 & k & [1] Table 78 \\
\hline Dowex $1 \times 8$ & $\mathrm{Cl}^{-}$ & - & $"$ & Swollen in water & $8 \times 10^{8}$ & - & 2.9 & k & $"$ \\
\hline Dowex $1 \times 4$ & $\mathrm{No}_{3}^{-}$ & - & $"$ & Moist & $1.1 \times 10^{8}$ & - & 3.7 & k & $"$ \\
\hline Dowex $1 \times 8$ & $\mathrm{OH}^{-}$ & 3.80 & $\begin{array}{l}\gamma \text {-radiation from } \\
\text { spent }{ }_{5} \text { reactor fuels } \\
\left(3 \times 10^{5}\right)\end{array}$ & Mo1st ( $52.4 \%$ water) & $1.06 \times 10^{8}$ & 35 & - & $\begin{array}{l}a, b, c, h, 1, \\
k, w\end{array}$ & {$[22]$} \\
\hline Dowex $1 \times 8$ & $\mathrm{OH}^{-}$ & 3.80 & $"$ & Mo1st ( $46.9 \%$ water) & $1.69 \times 10^{8}$ & 43.3 & - & $"$ & $"$ \\
\hline Dowex $1 \times 8$ & $\mathrm{OH}^{-}$ & 3.80 & $"$ & Moist ( $31.7 \%$ water) & $2.67 \times 10^{8}$ & 58.4 & - & $"$ & $"$ \\
\hline Dovex $1 \times 8$ & $\mathrm{Cl}^{-}$ & 2.31 & $"$ & $\begin{array}{l}\text { Alr-dried } \\
(41.3 \% \text { water })\end{array}$ & $0.76 \times 10^{8}$ & 22.3 & - & $\begin{array}{l}a, b, c, h, i \\
k, m\end{array}$ & {$[22]$} \\
\hline Dowex $1 \times 8$ & $\mathrm{Cl}^{-}$ & 2.31 & $"$ & $\begin{array}{l}\text { Immersed in water } \\
\text { ( } 43.7 \% \text { water })\end{array}$ & $0.87 \times 10^{8}$ & 27.6 & - & $"$ & $"$ \\
\hline Dowex $1 \times 4$ & $\mathrm{NO}_{3}^{-}$ & 2.79 & 60 & Moist ( $49.4 \%$ water) & $1.10 \times 10^{8}$ & 13.7 & - & $\begin{array}{l}a, b, c, h, 1, \\
k, m\end{array}$ & {$[22]$} \\
\hline Dowex $1 \times 4$ & $\mathrm{No}_{3}^{-}$ & 2.28 & $\begin{array}{l}\gamma \text {-radiation }\left({ }^{60} \mathrm{Co}\right) \\
\left(1.2 \times 10^{6}\right)\end{array}$ & $\begin{array}{l}\text { A1r-dried } \\
\quad(47.4 \% \text { water })\end{array}$ & $0.81 \times 10^{8}$ & 17.1 & - & $c, h, k, n$ & [24] \\
\hline Dowex $1 \times 4$ & $\mathrm{NO}_{3}^{-}$ & 2.28 & $"$ & "(49.3\%") & $1,11 \times 10^{8}$ & 13.6 & - & $"$ & $"$ \\
\hline$"$ & $\mathrm{No}_{3}^{-}$ & 2.28 & $"$ & $"(47.1 \% ")$ & $1.41 \times 10^{8}$ & 23.2 & - & $"$ & $"$ \\
\hline " & $\mathrm{NO}_{3}^{-}$ & 2.28 & $"$ & $"(42.3 \% ")$ & $1.52 \times 10^{8}$ & 16.2 & - & $"$ & $"$ \\
\hline$"$ & $"$ & $"$ & $"$ & $"(48.27 ")$ & $1.71 \times 10^{8}$ & 19.7 & - & $"$ & $"$ \\
\hline$"$ & $"$ & $"$ & $"$ & $"(49.62 ")$ & $2.01 \times 10^{8}$ & 22.3 & - & $"$ & $"$ \\
\hline$"$ & $"$ & $"$ & $"$ & $"(48.6 \% ")$ & $2.49 \times 10^{8}$ & 23.2 & - & $"$ & $"$ \\
\hline$"$ & $"$ & $"$ & $"$ & $"(46.6 \% ")$ & $3.68 \times 10^{8}$ & 27.6 & - & $"$ & $"$ \\
\hline$"$ & $"$ & $"$ & $"$ & $"(46.5 \% ")$ & $4.35 \times 10^{8}$ & 4.8 & - & $"$ & $"$ \\
\hline$"$ & $"$ & $"$ & $"$ & $"\left(\begin{array}{ll}42.67 & 1)\end{array}\right.$ & $6.00 \times 10^{8}$ & 36.4 & - & $"$ & $"$ \\
\hline " & $"$ & $"$ & $"$ & $"(41.2 \% ")$ & $6.90 \times 10^{8}$ & 38.2 & - & $"$ & $"$ \\
\hline Dowex $1 \times 4$ & $\mathrm{Cl}^{-}$ & 2.28 & $\begin{array}{l}\text { Y-radiation }{ }^{60} \mathrm{Co} \\
\left(1.2 \times 10^{6}\right)\end{array}$ & $\begin{array}{l}\text { Air-dried } \\
\quad(46.9 \% \text { water })\end{array}$ & $0.91 \times 10^{8}$ & 39.4 & - & $c, h, k, n$ & [24] \\
\hline$"$ & $"$ & $"$ & $"$ & $"(46.67 ")$ & $1.20 \times 10^{8}$ & 60.1 & - & $"$ & $"$ \\
\hline$"$ & $"$ & $"$ & $"$ & "(42.3\% ") & $1.71 \times 10^{8}$ & 41.7 & - & $"$ & $"$ \\
\hline " & $"$ & $"$ & $"$ & $"(38.7 \% ")$ & $2.16 \times 10^{8}$ & 53.9 & - & $"$ & $"$ \\
\hline$"$ & $"$ & $"$ & $"$ & $"\left(\begin{array}{ll}45.7 \% & 0\end{array}\right)$ & $2.70 \times 10^{8}$ & 57.5 & - & $"$ & $"$ \\
\hline$"$ & $"$ & $"$ & $"$ & $"(37.17 ")$ & $3.00 \times 10^{8}$ & 56.1 & - & $"$ & $"$ \\
\hline Dowex $1 \times 4$ & $\mathrm{No}_{3}^{-}$ & 2.28 & $\begin{array}{l}\text { Y-radiation }{ }^{60} \mathrm{Co} \\
\left(1.2 \times 10^{6}\right)\end{array}$ & $\begin{array}{l}\text { In } 7 \mathrm{~N} \mathrm{HNO}_{3} \\
(52.0 \% \text { water })\end{array}$ & $0.81 \times 10^{8}$ & 19.7 & - & $c, h, k, n$ & [24] \\
\hline 1 & $"$ & $"$ & $"$ & $"(56.52 \quad ")$ & $1.11 \times 10^{8}$ & 28.5 & - & $"$ & $"$ \\
\hline
\end{tabular}




\begin{tabular}{|c|c|c|c|c|c|c|c|c|c|c|c|}
\hline $\begin{array}{c}\text { RESIN } \\
\text { (Trade Name) }\end{array}$ & IONIC FORM & $\begin{array}{l}\text { EXCHANGE } \\
\text { CAPACITY } \\
\text { (meq } / \mathrm{g} \text { ) } \\
\end{array}$ & $\begin{array}{l}\text { RADIATION SOURCE } \\
\text { (Rad/Hr.) }\end{array}$ & \multicolumn{3}{|c|}{$\begin{array}{l}\text { IRRADLATION } \\
\text { CONDITION (S) }\end{array}$} & $\begin{array}{l}\text { INTEGRAL } \\
\text { DOSE } \\
\text { (RAD) } \\
\end{array}$ & $\begin{array}{c}\text { LOSS OF } \\
\text { EXCHANGE } \\
\text { CAPACITY } \\
(\%) \\
\end{array}$ & G VALUE & $\begin{array}{c}\text { OTHER } \\
\text { PARAMETERS } \\
\text { MEASURED } \\
\end{array}$ & $\begin{array}{l}\text { REFERENCE } \\
\text { NUMBER } \\
\text { AND NOTES } \\
\end{array}$ \\
\hline$"$ & $"$ & $"$ & $"$ & $"(59.0 \%$ & $"$ & ) & $1.41 \times 10^{8}$ & 39.9 & - & $"$ & $"$ \\
\hline$"$ & $"$ & $"$ & $"$ & $"(60.7 \%$ & $"$ & ) & $1.52 \times 10^{8}$ & 36.0 & - & $"$ & $"$ \\
\hline$"$ & $"$ & $"$ & $"$ & $"(61.5 \%$ & $"$ & ) & $1.71 \times 10^{8}$ & 53.5 & - & $"$ & $"$ \\
\hline$"$ & $"$ & $"$ & $"$ & $"(65.9 \%$ & $"$ & ) & $2.01 \times 10^{8}$ & 52.2 & - & $"$ & $"$ \\
\hline$"$ & $"$ & $"$ & $"$ & $"(71.2 \%$ & $" r$ & ) & $2.49 \times 10^{8}$ & 64.9 & - & $"$ & $"$ \\
\hline$"$ & $"$ & $"$ & $"$ & $"(79.6 \%$ & $")$ & ) & $3.68 \times 10^{8}$ & 78.9 & - & $"$ & $"$ \\
\hline$"$ & " & $"$ & $"$ & $"(91.4 \%$ & $"$, & ) & $4.35 \times 10^{8}$ & 36.8 & - & $"$ & $"$ \\
\hline Dowex 1 & $\mathrm{OH}^{-}$ & 5.35 & $x$-ray machine $\left(210^{6}\right)$ & Air-dry & & & $21 \times 10^{9}$ & 43.8 & - & $a, d, g, i, k$ & {$[37]$} \\
\hline Dowex $1 \times 1$ & $\mathrm{OH}^{-}$ & 5.23 & $x$-ray machine $\left(210^{6}\right)$ & Air-dry & & & $25 \times 10^{8}$ & 41 & - & $a, d, g, i, k$ & [37] \\
\hline Dowex $1 \times 2$ & $\mathrm{OH}^{-}$ & 6.25 & $"$ & $"$ & & & $"$ & 56 & - & Ir & $"$ \\
\hline Dowex $1 \times 4$ & $\mathrm{OH}^{-}$ & 5.94 & $"$ & $"$ & & & $"$ & 66 & - & 11 & $"$ \\
\hline Dowex $I \times 8$ & $\mathrm{OH}^{-}$ & 4.21 & $"$ & $"$ & & & $"$ & 56 & - & $"$ & $"$ \\
\hline Dowex $1 \times 10$ & $\mathrm{OH}^{-}$ & 4.19 & $"$ & $"$ & & & $"$ & 48 & . & $"$ & $"$ \\
\hline Dowex 1 & $\mathrm{Cl}^{-}$ & - & $x$-ray machine $\left(210^{6}\right)$ & Air-dry & & & $\sim 5 \times 10^{8}$ & 60 & - & $a, d, g, i, k$ & {$[37]$} \\
\hline Dowex 1 & $\mathrm{OH}^{-}$ & - & $"$ & $"$ & & & $"$ & 49 & - & $"$ & $"$ \\
\hline Dowex 2 & $\mathrm{Cl}^{-}$ & 2.1 & $\begin{array}{l}{ }^{60} \mathrm{Co} \text { radiations } \\
\left(21.9 \times 10^{5}\right)\end{array}$ & Air-dry & & & $3.2 \times 10^{7}$ & - & 14 & - & (1) Table 70 \\
\hline Dowex 2 & $\mathrm{OH}^{-}$ & 2.1 & $"$ & $"$ & & & $"$ & - & 20 & - & $"$ \\
\hline Dowex 2 & $\mathrm{Cl}^{-}$ & 2.10 & $y$-radiation $\left({ }^{60} \mathrm{Co}\right)$ & Dry resin & & & $0.02 \times 10^{7}$ & 6.7 & - & $i, k$ & {$[12]$} \\
\hline Dowex 2 & $\mathrm{Cl}^{-}$ & 2.10 & $\left(2 \times 10^{5}\right)$ & $"$ & & & $0.3 \times 10^{7}$ & 4.8 & - & - & $"$ \\
\hline Dowex 2 & $\mathrm{Cl}^{-}$ & 2.10 & $"$ & $"$ & & & $0.4 \times 10^{7}$ & 5.2 & - & - & $"$ \\
\hline Dowex 2 & $\mathrm{Cl}^{-}$ & 2.10 & $"$ & $"$ & & & $3.54 \times 10^{7}$ & 23.3 & - & - & $"$ \\
\hline Dowex 2 & $\mathrm{OH}^{-}$ & 2.10 & y-radiation $\left(2 \times 10^{5}\right)$ & Dry resin & & & $\begin{array}{l}0.02 \text { to } \\
3.54 \times 10^{7}\end{array}$ & $\begin{array}{l}10.5 \text { to } \\
33.3\end{array}$ & - & $i, k$ & [12] \\
\hline Dowex 3 & $\mathrm{OH}^{-}$ & 5.5 & $x$-ray unit $\left(1.1 \times 10^{8}\right)$ & Alr $r d r y$ & & & $2.2 \times 10^{9}$ & - & 3 & - & [1] Table 70 \\
\hline Dowex 3 & $\mathrm{Cl}^{-}$ & 2.45 & $\gamma$-radiation $\left(2 \times 10^{5}\right)$ & Dry resin & & & $\begin{array}{l}0.02 \text { to } \\
3.54 \times 10^{7}\end{array}$ & $\begin{array}{l}-0.8 \text { to } \\
16.1\end{array}$ & - & $i, k$ & [12] \\
\hline Dowex 3 & $\mathrm{OH}^{-}$ & 5.58 & $x$-ray machine $\left(210^{6}\right)$ & Air-dry & & & $21 \times 10^{9}$ & 19 & - & $a, d, g, i, k$ & [37] \\
\hline Dowex 11 & $\mathrm{NO}_{3}^{-}$ & 2.12 & $\begin{array}{l}\gamma \text {-radiation }\left({ }^{60} \mathrm{Co}\right) \\
\left(0.65 \times 10^{6}\right)\end{array}$ & $\begin{array}{l}\text { A1r-dried } \\
\quad(51.7 \pi\end{array}$ & ater) & & $1.56 \times 10^{8}$ & 22.6 & - & $c, f, g, k$ & {$[26][27]$} \\
\hline " & $"$ & $"$ & $"$ & $"(49.02$ & $")$ & & $3.12 \times 10^{8}$ & 42.9 & - & $"$ & $"$ \\
\hline$"$ & $"$ & $"$ & $"$ & $"(45.22$ & $"$ ) & ) & $4.68 \times 10^{8}$ & 57.1 & - & $"$ & $"$ \\
\hline " & $"$ & $"$ & $"$ & $"(51.87$ & $"$ ) & & $5.62 \times 10^{8}$ & 64.2 & - & $"$ & $"$ \\
\hline Dowex 11 & $\mathrm{NO}_{3}^{-}$ & 2.12 & $\begin{array}{l}\text { Y-radiatjon }\left({ }^{60} \mathrm{Co}\right) \\
\left(0.65 \times 10^{6}\right)\end{array}$ & $\begin{array}{l}\text { In } 7 \mathrm{~N} \mathrm{HNO}_{3} \\
\quad 68.18\end{array}$ & rater) & & $1.56 \times 10^{8}$ & 54.2 & - & $c, f, 8, k$ & {$[26][27]$} \\
\hline$"$ & $"$ & $"$ & $"$ & " (82.0z & ") & & $3.12 \times 10^{8}$ & 83.0 & - & $"$ & $"$ \\
\hline$"$ & $"$ & $"$ & $"$ & $"(89.57$ & $"$ ) & & $4.68 \times 10^{8}$ & 93.4 & - & $"$ & $"$ \\
\hline Dowex $21 \mathrm{~K}$ & $\mathrm{NO}_{3}^{-}$ & 4.5 & ${ }^{60}$ Co Y-radiation & In $\mathrm{MM} \mathrm{HNO}_{3}$ & & & $2.8 \times 10^{8}$ & - & 18 & - & [1] Table 77 \\
\hline $\begin{array}{l}\text { Anberlite } \\
\text { IRA }-400\end{array}$ & $\mathrm{OH}^{-}$ & 3.2 & $x$-ray un1t $\left(1.1 \times 10^{8}\right)$ & A1r-dry & & & $2.2 \times 10^{9}$ & - & 3.6 & - & [1] Table 70 \\
\hline$"$ & $\mathrm{OH}^{-}$ & 3.2 & $\begin{array}{l}{ }^{60} \mathrm{Co} \text { and }{ }^{144} \mathrm{Ce}-\mathrm{Pr} \\
\text { radiations }(2.5 \text { to } \\
6.0) \times 10^{5}\end{array}$ & $A \mid r-d r y$ & & & $9 \times 10^{8}$ & - & $\begin{array}{l}1.6 \text { to } \\
3.2\end{array}$ & - & [1] Table 70 \\
\hline$"$ & $\mathrm{OH}^{-}$ & 3.2 & $"$ & Molst & & & $9 \times 10^{8}$ & - & $"$ & - & $"$ \\
\hline
\end{tabular}


TABLE IV (Cont'd)

\begin{tabular}{|c|c|c|c|c|c|c|c|c|c|}
\hline $\begin{array}{c}\text { RESIN } \\
\text { (Trade Name) }\end{array}$ & IONIC FORM & $\begin{array}{l}\text { EXCHANGE } \\
\text { CAPACITY } \\
\text { (meq/g) } \\
\end{array}$ & $\begin{array}{c}\text { RADIATION SOURCE } \\
\text { (Rad/Hr.) }\end{array}$ & $\begin{array}{l}\text { IRRADIATION } \\
\text { CONDITION (s) } \\
\end{array}$ & $\begin{array}{c}\text { INTEGRAL } \\
\text { DOSE } \\
\text { (RAD) } \\
\end{array}$ & $\begin{array}{l}\text { LOSS OF } \\
\text { EXCHANGE } \\
\text { CAPACITY } \\
(y)\end{array}$ & G VALUE & $\begin{array}{l}\text { OTHER } \\
\text { PARAMETERS } \\
\text { MEASURED } \\
\end{array}$ & $\begin{array}{c}\text { REFERENCE } \\
\text { NUNBER } \\
\text { AND NOTES } \\
\end{array}$ \\
\hline $\begin{array}{l}\text { Amberite } \\
\text { IRA-400 }\end{array}$ & $\mathrm{OD}^{-}$ & 3.3 & $\begin{array}{l}\text { B, } \gamma \text {-radiation from } \\
\text { activation and fis- } \\
\text { sion products } \\
\left(1.4 \times 10^{7}\right)\end{array}$ & $\begin{array}{l}\text { Molst (on-1ine } \\
\text { cleanup system of } D_{2} O \\
\text { moderator) }\end{array}$ & $\begin{array}{l}\text { up to } \\
2.0 \times 10^{8}\end{array}$ & 25 to 25 & & $k, m$ & {$[13][15]$} \\
\hline $\begin{array}{l}\text { Amberlfte } \\
\text { IRA-400 }\end{array}$ & - & 3.72 & $\begin{array}{l}182 \text { Ta- } \gamma-\text { radjations } \\
\left(4.0-4.2 \times 10^{5}\right)\end{array}$ & $\begin{array}{l}\text { Dried (Ar } 60^{\circ} \mathrm{C} \\
\text { for } 48 \mathrm{hrs})\end{array}$ & $1.62 \times 10^{6}$ & 1.6 & - & $c, h, 1, k$ & {$[34][35][36]$} \\
\hline$"$ & - & 3.72 & $"$ & $"$ & $1.61 \times 10^{7}$ & 0.8 & - & $"$ & $"$ \\
\hline$"$ & - & 3.72 & $"$ & Wet (filtered) & $2.13 \times 10^{6}$ & 5.4 & - & $"$ & $"$ \\
\hline$"$ & - & 3.72 & $"$ & $"$ & $1.62 \times 10^{7}$ & 0.8 & - & $"$ & $"$ \\
\hline$"$ & - & 3.72 & $\begin{array}{l}60 \text { Co- }- \text {-radiat 1ons } \\
\left(4.2 \times 10^{5}\right)\end{array}$ & $"$ & $1.75 \times 10^{8}$ & 20.2 & - & $"$ & $"$ \\
\hline $\begin{array}{l}\text { Amber11ce } \\
\text { IRA-400 }\end{array}$ & $\mathrm{OH}^{-}$ & 5.30 & $x$-ray achine $\left(210^{6}\right)$ & Alt-dry & $\sim_{1 \times 10^{9}}$ & 42.1 & - & $a, d, 8,1, k$ & [37] \\
\hline $\begin{array}{l}\text { Amberlite } \\
\text { IRA-401 }\end{array}$ & $\mathrm{OH}^{-}$ & 3.5 & $\begin{array}{l}{ }^{60} \mathrm{Co} \text { and }{ }^{144} \mathrm{Ce}-\mathrm{Pr} \\
\text { radiatigns }(2.5 \text { to } \\
6.0) \times 10^{5}\end{array}$ & Air-dry & $9 \times 10^{8}$ & - & $\begin{array}{l}1.8 \text { to } \\
3.6\end{array}$ & - & [1] Table 70 \\
\hline " & $\mathrm{OH}^{-}$ & 2.15 & Reactor $\left(2.6 \times 10^{6}\right)$ & Alr-dry & $1.1 \times 10^{9}$ & - & 1.3 & - & $"$ \\
\hline$"$ & $\mathrm{OH}^{-}$ & 3.5 & $\begin{array}{l}{ }^{60} \mathrm{Co} \text { and }{ }^{144} \mathrm{Ce}-\mathrm{Pr} \\
\text { radiatigns }(2.5 \text { to } \\
6.0) \times 10^{5}\end{array}$ & Moist & $9 \times 10^{8}$ & - & $\begin{array}{l}1.8 \text { to } \\
3.6\end{array}$ & - & [1] Table 77 \\
\hline " & $\mathrm{NO}_{3}^{-}$ & 3.5 & ${ }^{60}$ Co $\gamma$-radiation & In $7 \mathrm{M} \mathrm{HNO}_{3}$ & $4.2 \times 10^{8}$ & - & 5.5 & - & [1] Table 77 \\
\hline $\begin{array}{l}\text { Amberl1te } \\
\text { IRA-401 }\end{array}$ & - & 3.81 & ${ }^{182} \mathrm{Ta}$ Y-radiations & Dry & $1.62 \times 10^{6}$ & -0.8 & - & $c, h, 1, k$ & {$[34][35][36]$} \\
\hline$"$ & - & 3.81 & $"$ & $"$ & $1.61 \times 10^{7}$ & -1.6 & - & " & $"$ \\
\hline$"$ & - & 3.81 & $"$ & Wet (filtered) & $1.66 \times 10^{8}$ & -3.4 & - & $"$ & $"$ \\
\hline$"$ & - & 3.81 & $"$ & $"$ & $2.13 \times 10^{6}$ & 6.6 & - & $"$ & 11 \\
\hline$"$ & - & 3.81 & ${ }^{60}$ Co r-radiations & $"$ & $1.75 \times 10^{8}$ & 26.8 & - & $"$ & $"$ \\
\hline $\begin{array}{l}\text { Amberlite } \\
\text { IRA-410 }\end{array}$ & $\mathrm{OH}^{-}$ & 3.5 & $x$-ray unit $\left(1.1 \times 10^{8}\right)$ & Air-dry & $2.2 \times 10^{9}$ & - & 4.0 & - & [1] Table 70 \\
\hline$"$ & $\mathrm{OH}^{-}$ & 3.4 & $\begin{array}{l}{ }^{60} \mathrm{Co} \text { and }{ }^{144} \mathrm{Ce}-\mathrm{Pr} \\
\text { radiations }(2.5 \text { to } \\
6.0) \times 10^{5}\end{array}$ & Air-dry & $9 \times 10^{8}$ & - & $\begin{array}{l}1.7 \text { to } \\
3.4\end{array}$ & - & $"$ \\
\hline$"$ & $\mathrm{OH}^{-}$ & 3.4 & $"$ & Mo1st & $9 \times 10^{8}$ & - & $\begin{array}{l}1.7 \text { to } \\
3.4\end{array}$ & - & [1] Table 77 \\
\hline $\begin{array}{l}\text { Auberlite } \\
\text { IRA-410 }\end{array}$ & - & 3.88 & $\begin{array}{l}182 \text { Ta } \gamma \text {-radiation } \\
\text { (4 to } 4.2 \times 10^{5} \text { ) }\end{array}$ & Dry & $1.62 \times 10^{6}$ & -4.1 & - & $c, h, 1, k$ & {$[34][35](36)$} \\
\hline$"$ & - & 3.88 & $"$ & $"$ & $1.61 \times 10^{7}$ & 0 & - & $"$ & $"$ \\
\hline$"$ & - & 3.88 & $\begin{array}{l}{ }^{60} \mathrm{Co} \text { r-radiation } \\
\left(4.2 \times 10^{5}\right)\end{array}$ & $"$ & $1.66 \times 10^{8}$ & 20.1 & - & $"$ & $"$ \\
\hline$"$ & - & 3.88 & $\begin{array}{l}182 \text { Ta } \gamma \text {-radiation } \\
\left(4 \text { to } 4.2 \times 10^{5}\right)\end{array}$ & Wet (filtered) & $2.13 \times 10^{6}$ & 0.3 & - & $"$ & $"$ \\
\hline$"$ & - & 3.88 & $"$ & $"$ & $1.73 \times 10^{7}$ & -2.3 & - & $"$ & $"$ \\
\hline $\begin{array}{l}\text { Amberl1te } \\
\text { IRA-410 }\end{array}$ & $\mathrm{OH}^{-}$ & 4.89 & $x$-ray machine $\left(\sim 10^{6}\right)$ & Alr-dry & $21 \times 10^{9}$ & 39.6 & - & $a, d, g, 1, k$ & [37] \\
\hline $\begin{array}{l}\text { Amber11te } \\
\text { IRA-411 }\end{array}$ & $\mathrm{OH}^{-}$ & $3 .{ }^{\prime} 0$ & $\begin{array}{l}{ }^{60} \mathrm{Co} \text { and }{ }^{144} \mathrm{Ce}-\mathrm{Pr} \\
\text { radiatigns }(2.5 \text { to } \\
6.0) \times 10^{5}\end{array}$ & Alr-dry & $9 \times 10^{8}$ & - & $\begin{array}{l}1.5 \\
\text { to } 3.0\end{array}$ & - & [1] Table 70 \\
\hline$"$ & $\mathrm{OH}^{-}$ & 3.0 & $"$ & Mo1st & $9 \times 10^{8}$ & - & $\begin{array}{l}1.5 \\
\text { to } 3.0\end{array}$ & - & [1] Table 77 \\
\hline $\begin{array}{l}\text { Amber11te } \\
\text { 1RA-411 }\end{array}$ & - & 3.70 & $\begin{array}{l}182 \text { Ta Y-radiations } \\
\left(4 \text { to } 4.2 \times 10^{5}\right)\end{array}$ & Dry & $1.62 \times 10^{6}$ & 5.4 & - & $c, h, 1, k$ & {$[34][35][36]$} \\
\hline$"$ & - & 3.70 & $"$ & $"$ & $1.61 \times 10^{7}$ & 7.6 & - & $"$ & + \\
\hline$"$ & - & 3.70 & $"$ & Wet -(filtered) & $2.13 \times 10^{6}$ & 5.7 & - & $"$ & $"$ \\
\hline$"$ & - & 3.70 & $"$ & $"$ & $1.73 \times 10^{7}$ & 9.5 & - & $"$ & $"$ \\
\hline$"$ & - & 3.70 & $\begin{array}{l}{ }^{60} \text { Co } \gamma \text {-radiations } \\
\left(4.2 \times 10^{5}\right)\end{array}$ & . " - & $1.71 \times 10^{8}$ & 29.5 & - & $"$ & $"$ \\
\hline
\end{tabular}




\begin{tabular}{|c|c|c|c|c|c|c|c|c|c|}
\hline $\begin{array}{c}\text { RESIN } \\
\text { (Trade Name) } \\
\end{array}$ & IONIC FORM & $\begin{array}{l}\text { EXCHANGE } \\
\text { CAPACI TY } \\
\text { (meq/8) } \\
\end{array}$ & $\begin{array}{c}\text { RADIATION SOURCE } \\
(\mathrm{Rad} / \mathrm{Hr} .)\end{array}$ & $\begin{array}{l}\text { IRRADIATION } \\
\text { CONDITION(S) } \\
\end{array}$ & $\begin{array}{c}\text { INTEGRAL } \\
\text { DOSE } \\
\text { (RAD) } \\
\end{array}$ & $\begin{array}{c}\text { LOSS OF } \\
\text { EXCHANGE } \\
\text { CAPACITY } \\
(\mathrm{z}) \\
\end{array}$ & G VALUE & $\begin{array}{c}\text { OTHER } \\
\text { PARAMETERS } \\
\text { MEASURED } \\
\end{array}$ & $\begin{array}{c}\text { REFERENCE } \\
\text { NUMBER } \\
\text { AND NOTES } \\
\end{array}$ \\
\hline Amberlite XE- 58 & $\mathrm{OH}^{-}$ & 8.0 & X-ray unit $\left(1.1 \times 10^{8}\right)$ & A1t-dry & $2.2 \times 10^{9}$ & - & 5.3 & - & [1] Table 70 \\
\hline Amberlite $\mathrm{XE}-58$ & $\mathrm{OH}^{-}$ & 6.01 & $x$-ray machine $\left(\sim 10^{6}\right)$ & $\begin{array}{l}\text { Powdered Air-dry } \\
\text { sample }\end{array}$ & $21 \times 10^{9}$ & 20 & - & $a, d, 8,1, k$ & [37] \\
\hline Amberlite IR-4B & $\mathrm{OH}^{-}$ & 9.2 & X-ray unit $\left(1.1 \times 10^{8}\right)$ & A1r-dry & $2.2 \times 10^{9}$ & - & 3.3 & - & [1] Table 70 \\
\hline Amberlite IR-4B & - & 10.85 & $\begin{array}{l}{ }^{182} \mathrm{Ta} \gamma \text {-radiations } \\
\left(4 \text { to } 4.2 \times 10^{5}\right)\end{array}$ & Dry & $1.62 \times 10^{6}$ & -0.5 & - & $c, h, 1, k$ & {$[34][35](36]$} \\
\hline$"$ & - & 10.85 & $"$ & $"$ & $1.61 \times 10^{7}$ & 0.9 & - & $"$ & $"$ \\
\hline$"$ & - & 10.85 & $\begin{array}{l}{ }^{60} \text { Co y-radiations } \\
\left(4.2 \times 10^{5}\right)\end{array}$ & $"$ & $1.72 \times 10^{8}$ & 6.0 & - & $"$ & $"$ \\
\hline$"$ & - & 10.85 & $\begin{array}{l}182 \text { Ta } \gamma \text {-radiations } \\
\left(4.0 \text { to } 4.2 \times 10^{5}\right)\end{array}$ & Wet (filtered) & $1.68 \times 10^{6}$ & 9.2 & - & $"$ & $"$ \\
\hline$"$ & - & 10.85 & $"$ & $"$ & $1.73 \times 10^{7}$ & 16.1 & - & $"$ & $"$ \\
\hline$"$ & - & 10.85 & $\begin{array}{l}60 \mathrm{Co} \gamma-\mathrm{rad1at10ns} \\
\left(4.2 \times 10^{5}\right)\end{array}$ & $"$ & $1.73 \times 10^{8}$ & 18.0 & - & $"$ & $"$ \\
\hline Amberlfte IR-4B & $\mathrm{OH}^{-}$ & 8.32 & $x$-ray machlne $\left(210^{6}\right)$ & A1r-dry & $\sim 1 \times 10^{9}$ & 13 & - & $a, d, 8,1, k$ & [37] \\
\hline Amber11te IR-45 & $\mathrm{OH}^{-}$ & 5.5 & $x$-ray unit $\left(1.1 \times 10^{8}\right)$ & $A 1 r-d r y$ & $2.2 \times 10^{9}$ & - & 8.0 & - & (1) Table 70 \\
\hline Amberlite IR-45 & - & 5.10 & $\begin{array}{l}182 \mathrm{Ta} \text { y-radiations } \\
\left(4 \text { to } 4.2 \times 10^{5}\right)\end{array}$ & Dry & $1.61 \times 10^{6}$ & 2.4 & - & $c, h, 1, k$ & {$[34][35][36]$} \\
\hline$"$ & - & 5.10 & $"$ & $"$ & $1.53 \times 10^{7}$ & 2.9 & - & $"$ & $"$ \\
\hline$"$ & - & 5.10 & ${ }^{60} \mathrm{Co}$ y-radiat Ions & $"$ & $1.72 \times 10^{8}$ & 25.1 & - & $"$ & $"$ \\
\hline$"$ & - & 5.10 & $\begin{array}{l}182 \mathrm{Ta} \text {-radiations } \\
\left(4 \text { to } 4.2 \times 10^{5}\right)\end{array}$ & Wet (filtered) & $1.68 \times 10^{6}$ & 1.0 & - & " & $"$ \\
\hline$"$ & - & 5.10 & $"$ & $"$ & $1.72 \times 10^{7}$ & 8.6 & - & $"$ & $"$ \\
\hline$"$ & - & 5.10 & $\begin{array}{l}{ }^{60} \text { Co } \gamma \text {-radiations } \\
\left(4.2 \times 10^{5}\right)\end{array}$ & $"$ & $1.73 \times 10^{8}$ & 12.7 & - & $"$ & $"$ \\
\hline Amberlite IR-45 & $\mathrm{OH}^{-}$ & 6.35 & X-ray mach1ne $\left(210^{6}\right)$ & Alr-dry & $21 \times 10^{9}$ & 53 & - & $a, d, 8,1, k$ & [37] \\
\hline Amberl1te XE-114 & - & 9.50 & $\begin{array}{l}182 \mathrm{Ta} \text {-radiations } \\
\left(4 \text { to } 4.2 \times 10^{5}\right)\end{array}$ & Dry & $1.61 \times 10^{6}$ & -1.3 & - & $c, h, 1, k$ & {$[34][35][36]$} \\
\hline$"$ & - & 9.50 & $"$ & $"$ & $1.53 \times 10^{7}$ & 4.7 & - & $"$ & $"$ \\
\hline$"$ & - & 9.50 & $\begin{array}{l}60 \mathrm{Co} \text { r-radiatione } \\
\left(4.2 \times 10^{5}\right)\end{array}$ & $"$ & $1.72 \times 10^{8}$ & 14.2 & - & $"$ & $"$ \\
\hline$"$ & - & 9.50 & $\begin{array}{l}182 \mathrm{Ta} \text { y-radjactiona } \\
\left(4.0-4.2 \times 10^{5}\right)\end{array}$ & Wet (f1ltered) & $1.68 \times 10^{6}$ & 0.4 & - & $"$ & $"$ \\
\hline$"$ & - & 9.50 & $"$ & $"$ & $1.72 \times 10^{7}$ & 18.3 & - & $"$ & $"$ \\
\hline$"$ & - & 9.50 & $\begin{array}{l}{ }^{60} \text { Co } \gamma \text {-radiations } \\
\left(4.2 \times 10^{5}\right)\end{array}$ & $"$ & $1.73 \times 10^{8}$ & 28.9 & - & $"$ & $"$ \\
\hline Imac $s-4$ & $\mathrm{OH}^{-}$ & 1.5 & Reactor $\left(2.6 \times 10^{6}\right)$ & Air-dry & $1.1 \times 10^{9}$ & - & 1.1 & - & [1] Table 70 \\
\hline Imac $55-40$ & $\mathrm{OH}^{-}$ & 3.62 & $\begin{array}{l}\text { Y-radfation }\left({ }^{60} \mathrm{Co}\right) \\
\left(1 \times 10^{6}\right)\end{array}$ & $\begin{array}{l}\text { Reain Imersed in } \\
\text { water }\end{array}$ & $1 . \times 10^{7}$ & 9.9 & $\begin{array}{l}35 \\
\text { (radia- } \\
\text { tively } \\
\text { labile) }\end{array}$ & $h, k, 1, m$ & $\begin{array}{l}(20] \\
\text { (Decompos1- } \\
\text { tion follows } \\
\text { the first } \\
\text { order kinett } \\
\text { equation) }\end{array}$ \\
\hline$"$ & $"$ & $"$ & $"$ & $"$ & $2 \times 10^{7}$ & 13.3 & - & $"$ & $"$ \\
\hline$"$ & $"$ & " & $"$ & $"$ & $3 \times 10^{7}$ & 14.1 & - & $"$ & $"$ \\
\hline " & $"$ & $"$ & $"$ & $"$ & $4 \times 10^{7}$ & 11.1 & - & $"$ & $"$ \\
\hline$"$ & $"$ & $"$ & $"$ & $"$ & $8 \times 10^{7}$ & 16.3 & - & $"$ & $"$ \\
\hline$"$ & $"$ & $"$ & $"$ & $"$ & $1 \times 10^{8}$ & 17.1 & $\begin{array}{l}7 \\
\text { (radia- } \\
\text { tively } \\
\text { more } \\
\text { Btable) }\end{array}$ & $w$ & $"$ \\
\hline
\end{tabular}


TABLE IV (Cont $\left.{ }^{*} d\right)$

\begin{tabular}{|c|c|c|c|c|c|c|c|c|c|c|}
\hline $\begin{array}{r}\text { RES } \\
\text { (Trade }\end{array}$ & Name) & IONIC PORM & $\begin{array}{l}\text { EXCHANGE } \\
\text { CAPACITY } \\
\text { (meg } / g \text { ) }\end{array}$ & $\begin{array}{l}\text { RADIATION SOURCE } \\
(\mathrm{Rad} / \mathrm{HI},)\end{array}$ & $\begin{array}{l}\text { LRRADIATION } \\
\text { CONDITION (S) }\end{array}$ & $\begin{array}{c}\text { INTEGRAL } \\
\text { DOSE } \\
\text { (RAD) } \\
\end{array}$ & $\begin{array}{c}\text { LOSS OF } \\
\text { EXCHANGE } \\
\text { CAPACITY } \\
(\%)\end{array}$ & G VALUE & $\begin{array}{c}\text { OTHER } \\
\text { PARAMETERS } \\
\text { MEASURED } \\
\end{array}$ & $\begin{array}{c}\text { REFERENCE } \\
\text { NUMBER } \\
\text { AND NOTES } \\
\end{array}$ \\
\hline$"$ & & $"$ & $"$ & $"$ & $"$ & $2.15 \times 10^{8}$ & 35.1 & - & $"$ & $"$ \\
\hline$"$ & & $"$ & $"$ & $"$ & $"$ & $3.05 \times 10^{8}$ & 47.5 & - & $n$ & $"$ \\
\hline$"$ & & $"$ & $"$ & $"$ & $"$ & $4.15 \times 10^{8}$ & 51.1 & - & $"$ & $"$ \\
\hline$"$ & & $"$ & $"$ & $"$ & $"$ & $6.0 \times 10^{8}$ & 64.6 & - & $"$ & $"$ \\
\hline$"$ & & $"$ & $"$ & $"$ & $"$ & $8.0 \times 10^{8}$ & 79.0 & - & $"$ & $"$ \\
\hline$"$ & & $"$ & $"$ & $"$ & $"$ & $1.0 \times 10^{9}$ & 88.7 & - & $"$ & $"$ \\
\hline$"$ & & $"$ & $"$ & $"$ & $"$ & $1.5 \times 10^{9}$ & 90.1 & - & $"$ & $"$ \\
\hline$"$ & & $"$ & $"$ & $"$ & $"$ & $2.0 \times 10^{9}$ & 97.2 & - & $"$ & $"$ \\
\hline Zerolit & $\begin{array}{l}\text { FF-IP } \\
X 7-9\end{array}$ & $\mathrm{Cl}^{-}$ & 2.9 & $\begin{array}{l}\gamma \text {-radiation }{ }^{60} \mathrm{Co}^{6} \\
\left(0.43 \text { and } 1.0 \times 10^{6}\right)\end{array}$ & $\begin{array}{l}\text { Air-dried } \\
\quad(1.2 \% \text { water })\end{array}$ & $1 \times 10^{8}$ & 8.6 & 3.5 & $c, h, 1, k, 1$, & {$[16][17][19]$} \\
\hline$"$ & $x 7-9$ & $\mathrm{CI}^{-}$ & 2.9 & $"$ & 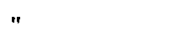 & $1.7 \times 10^{8}$ & 25.9 & 3.5 & $"$ & $"$ \\
\hline$"$ & $x 7-9$ & $\mathrm{Cl}^{-}$ & 2.9 & $"$ & $"$ & $2.0 \times 10^{8}$ & 31.0 & 3.5 & $"$ & $"$ \\
\hline$"$ & $x 7-9$ & $\mathrm{Cl}^{-}$ & 2.9 & $"$ & $"$ & $2.4 \times 10^{8}$ & 44.8 & 3.5 & $"$ & $"$ \\
\hline$"$ & $x 7-9$ & $\mathrm{CI}^{-}$ & 2.9 & $"$ & $"$ & $3.8 \times 10^{8}$ & 67.2 & 3.5 & $"$ & $"$ \\
\hline$"$ & $x 7-9$ & $\mathrm{Cl}^{-}$ & 2.9 & $"$ & $"$ & $1.0 \times 10^{9}$ & 82.8 & - & $"$ & $"$ \\
\hline $\begin{array}{l}\text { Zerol1t } \\
\text { FF-IP×2- }\end{array}$ & & $\mathrm{Cl}^{-}$ & 4.12 & $\begin{array}{l}\text { Y-radiation }{ }^{60} \mathrm{Co}^{6} \\
\left(0.43 \text { and } 1.0 \times 10^{6}\right)\end{array}$ & $\begin{array}{l}\text { A1r-dried } \\
\text { (17\% moisture) }\end{array}$ & $1 \times 10^{8}$ & 12.6 & 4.1 & $\varepsilon_{\text {m }}, h, i, k, l$, & {$[16](17][19]$} \\
\hline$"$ & & $\mathrm{Cl}^{-}$ & $"$ & $"$ & $"$ & $2 \times 10^{8}$ & 26.0 & 4.1 & $"$ & $"$ \\
\hline$"$ & & $\mathrm{Cl}^{-}$ & $"$ & $"$ & $"$ & $3 \times 10^{8}$ & 53.9 & 4.1 & $"$ & $"$ \\
\hline$"$ & & $\mathrm{Cl}^{-}$ & $"$ & $"$ & $"$ & $4.4 \times 10^{8}$ & 72.1 & 4.1 & $"$ & $"$ \\
\hline$"$ & & $\mathrm{Cl}^{-}$ & $"$ & $"$ & $"$ & $1 \times 10^{9}$ & 87.9 & - & $"$ & $"$ \\
\hline $\begin{array}{l}\text { Zerolit } \\
\text { FF-IP X7- }\end{array}$ & & $\mathrm{Cl}^{-}$ & 2.9 & $\begin{array}{l}\left.\gamma \text {-radiation }{ }^{60} \mathrm{Co}^{6}\right) \\
\left(0.43 \text { and } 1.0 \times 10^{6}\right)\end{array}$ & Wet resin & $4.05 \times 10^{8}$ & 68 & 5.4 & $c, h, i, k, 1$, & {$[16][17][19]$} \\
\hline$"$ & & $\mathrm{Cl}^{-}$ & 2.9 & $"$ & $"$ & $1.0 \times 10^{9}$ & 85.5 & - & $"$ & $"$ \\
\hline$"$ & & $\mathrm{Cl}^{-}$ & 2.9 & $"$ & $"$ & $1.5 \times 10^{9}$ & - & - & $"$ & $"$ \\
\hline $\begin{array}{l}\text { Zerol1t } \\
\text { FF-1Px2- }\end{array}$ & & $\mathrm{CI}^{2}$ & 4.12 & $\begin{array}{l}\text { r-radiation }{ }^{60} \mathrm{Co}^{6} \\
\left(0.43 \text { and } 1.0 \times 10^{6}\right)\end{array}$ & Wet resin & $4.05 \times 10^{8}$ & 81 & 8.9 & $\begin{array}{l}c, h, l, k, l, \\
m\end{array}$ & {$[16][17][19]$} \\
\hline$"$ & & $\mathrm{Cl}^{-}$ & 4.12 & $"$ & $"$ & $1.0 \times 10^{9}$ & 94 & - & $"$ & $"$ \\
\hline$"$ & & $\mathrm{Cl}^{-}$ & 4.12 & $"$ & $"$ & $1.5 \times 10^{9}$ & - & - & $"$ & $"$ \\
\hline$"$ & & $\mathrm{Cl}^{-}$ & 4.12 & $"$ & $"$ & $2.0 \times 10^{9}$ & - & - & $"$ & $"$ \\
\hline$"$ & & $\mathrm{Cl}^{-}$ & 4.12 & $"$ & $"$ & $2.5 \times 10^{9}$ & - & - & $"$ & $"$ \\
\hline $\begin{array}{l}\text { Zerolit } \\
\text { FF-IPx7- }\end{array}$ & & $\mathrm{Cl}^{-}$ & 2.9 & $\begin{array}{l}\gamma \text {-radiation }{ }^{60} \mathrm{Co} \\
\left(0.4 \text { and } 1.0 \times 10^{6}\right)\end{array}$ & Dry resin & $4.05 \times 10^{8}$ & 50 & 3.1 & $\begin{array}{l}c, h, i, k, l, \\
\mathrm{~m}\end{array}$ & {$[16][17][19]$} \\
\hline$"$ & & $\mathrm{Cl}^{-}$ & 2.9 & $"$ & $"$ & $1.0 \times 10^{9}$ & 66 & - & $"$ & $"$ \\
\hline$"$ & & $\mathrm{Cl}^{-}$ & 2.9 & $"$ & $"$ & $1.5 \times 10^{9}$ & 85 & - & 1 & $"$ \\
\hline $\begin{array}{l}\text { Zerolit } \\
\text { FF-IPx2- }\end{array}$ & & $\mathrm{Cl}^{-}$ & 4.12 & $\begin{array}{l}\text { r-radiation }{ }^{60} \mathrm{Co} \\
\left(0.4 \text { and } 1.0 \times 10^{6}\right)\end{array}$ & Dry resin & $4.05 \times 10^{8}$ & 45 & 3.4 & $c_{\text {m }}, h, 1, k, 1$, & {$[16][17][19]$} \\
\hline$"$ & & $\mathrm{Cl}^{-}$ & 4.12 & $"$ & $"$ & $1.0 \times 10^{9}$ & 55 & - & $"$ & $"$ \\
\hline$"$ & & $\mathrm{Cl}^{-}$ & 4.12 & $"$ & $"$ & $1.5 \times 10^{9}$ & 82 & - & $"$ & $"$ \\
\hline$"$ & & $\mathrm{Cl}^{-}$ & 4.12 & $"$ & $"$ & $2.0 \times 10^{9}$ & 88 & - & $"$ & $"$ \\
\hline$"$ & & $\mathrm{Cl}^{-}$ & 4.12 & $"$ & $"$ & $2.5 \times 10^{9}$ & 87 & - & $"$ & $"$ \\
\hline Dienon $\mathrm{S}$ & $S A-100$ & $\mathrm{Cl}^{-}$ & 2.06 & $\begin{array}{l}{ }^{60} \text { Co } y-\text { radiations } \\
\left(1.9 \times 10^{5}\right)\end{array}$ & Alr-dry & $3.2 \times 10^{7}$ & - & 8.5 & - & [1] Table 70 \\
\hline$"$ & & $\mathrm{OH}^{-}$ & 2.06 & $"$ & $"$ & $3.2 \times 10^{7}$ & - & 15 & - & [1] Table 70 \\
\hline Dialon $S$ & SAf100 & $\mathrm{Cl}^{-}$ & 2,06 & $\gamma$-radiation $\left(2 \times 10^{5}\right)$ & Dry resin & $\begin{array}{l}0.02 \text { to } \\
3.54 \times 10^{7}\end{array}$ & $\begin{array}{l}1.9 \\
\text { to } 14.1\end{array}$ & - & $1, k$ & [12] \\
\hline
\end{tabular}


TABLE IV (Cont'd)

\begin{tabular}{|c|c|c|c|c|c|c|c|c|c|}
\hline $\begin{array}{c}\text { RESIN } \\
\text { (Trade Name) } \\
\end{array}$ & IONIC FORM & $\begin{array}{l}\text { EXCHANCE } \\
\text { CAPACITY } \\
\text { (aeg/g) }\end{array}$ & $\begin{array}{c}\begin{array}{c}\text { RADIAT ION SOURCE } \\
\left(\mathrm{Rad} / \mathrm{H}_{\mathrm{r}} .\right)\end{array} \\
\end{array}$ & $\begin{array}{l}\text { IRRADIATION } \\
\text { CONDITION(S) } \\
\end{array}$ & $\begin{array}{c}\text { INTECRAL } \\
\text { DOSE } \\
\text { (RAD) } \\
\end{array}$ & $\begin{array}{l}\text { LOSS OF } \\
\text { EXCHANCE } \\
\text { CAPACITY } \\
\\
\end{array}$ & $\underline{G \text { VALUE }}$ & $\begin{array}{c}\text { OTHER } \\
\text { PARAMETERS } \\
\text { MEASURED } \\
\end{array}$ & $\begin{array}{c}\text { REFERENCE } \\
\text { NUMBER } \\
\text { AND NOTES } \\
\end{array}$ \\
\hline Diaion SAll 100 & $\mathrm{OH}^{-}$ & 2.06 & r-radiation $\left(2 \times 10^{5}\right)$ & Dry resin & " & $\begin{array}{l}10.5 \\
\text { to } 25.8\end{array}$ & - & $1, \mathrm{k}$ & [12] \\
\hline Nalcite SAR & $\mathrm{OH}^{-}$ & 3.9 & $x$-ray unit $\left(1.1 \times 10^{8}\right)$ & $\operatorname{A1r} \approx d r y$ & $2.2 \times 10^{9}$ & - & 4.0 & - & [1] Table 70 \\
\hline Nalcite SAR & $\mathrm{OH}^{-}$ & 3.78 & $x$-ray machine $\left(\eta 10^{6}\right)$ & Alr-dry & $21 \times 10^{9}$ & 37.0 & - & $a, d, 8,1, k$ & [37] \\
\hline Nalcite WBR & $\mathrm{OH}^{-}$ & 3.5 & $x$-ray un1e $\left(1.1 \times 10^{8}\right)$ & Air-dry & $2.2 \times 10^{9}$ & - & 2 & - & [1] Table 70 \\
\hline Nalcite $\mathrm{kBR}$ & $\mathrm{OH}^{-}$ & 6.01 & $x$-ray machine $\left(\sim 10^{6}\right)$ & $A 1 r-d r y$ & $21 \times 10^{9}$ & 20 & - & $a, d, g, 1, k$ & [37] \\
\hline Permutit SK & $\mathrm{OH}^{-}$ & - & $\gamma$-emitter & Molst & $2.7 \times 10^{8}$ & - & 4.6 & $k$ & [1] Table 78 \\
\hline Permut1t SK & $\mathrm{Cl}^{-}$ & - & " & $"$ & $10^{8}$ & - & 2.5 & $\mathrm{k}$ & " \\
\hline Permut1t SK & $\mathrm{OH}^{-}$ & 4.47 & $\begin{array}{l}\gamma \text {-radiation frod } \\
\text { spent reactor fuels }\end{array}$ & Holst (63.3z water) & $1.69 \times 10^{8}$ & 4.9 & - & $\begin{array}{l}a, b, c, h, 1, \\
k, m\end{array}$ & [22] \\
\hline Permut1t SK & $\mathrm{OH}^{-}$ & 4.47 & & Molst ( $61.4 \mathrm{Z}$ vater) & $2.67 \times 10^{8}$ & 7.8 & - & " & $"$ \\
\hline Permut1t SK & $\mathrm{Cl}^{-}$ & 4.47 & $"$ & Dry (62.8z vater) & $1.06 \times 10^{8}$ & 4.3 & - & $\begin{array}{l}a, b, c, h, 1, \\
k, m\end{array}$ & [22] \\
\hline Permut1t SK & $\mathrm{Cl}^{-}$ & 4.47 & $"$ & Molst ( $59.3 \%$ water) & $1.08 \times 10^{8}$ & 7.6 & - & " & $"$ \\
\hline Permut1t S-2 & $\mathrm{OH}^{-}$ & 3.4 & $x$-ray unit $\left(1.1 \times 10^{6}\right)$ & A1r-dry & $2.2 \times 10^{9}$ & - & 3.5 & - & [1] Table 70 \\
\hline Permutit S-2 & $\mathrm{OH}^{-}$ & 4.4 & $x$-ray machine $\left(? 10^{6}\right)$ & Alr-dry & $21 \times 10^{9}$ & 38.3 & - & $a, d, g, i, k$ & [37] \\
\hline $\begin{array}{l}\text { Deacidite } \\
\text { FFx7-9 }\end{array}$ & $\mathrm{OH}^{-}$ & 4.02 & $\begin{array}{l}\gamma \text {-radiafion }{ }^{60} \mathrm{Co} \\
\left(6.1 \times 10^{5}\right)\end{array}$ & Aqueous slurries & $5.5 \times 10^{7}$ & 12.2 & $\begin{array}{l}\text { G-value } \\
\text { for var- } \\
\text { lous } \\
\text { product } \\
\text { formation } \\
\text { reported }\end{array}$ & $b, h, k, 1$ & $\begin{array}{l}\text { [28] A1so in- } \\
\text { cluded in } \\
\text { Ref. [1] }\end{array}$ \\
\hline $\begin{array}{l}\text { Deac1dite } \\
\text { FF } 7 \text { 7-9 }\end{array}$ & $\mathrm{OH}^{-}$ & 4.02 & $\left(1.8 \times 10^{5}\right)$ & $"$ & $6.5 \times 10^{7}$ & 15.7 & & & \\
\hline Deacidi te FFx7-9 & $\mathrm{So}_{4}^{2-}, \mathrm{Cl}^{-}$ & & $\begin{array}{l}\text { Y-radiation }\left({ }^{60} \mathrm{co}\right) \\
\left(8.01 \times 10^{5}\right)\end{array}$ & Dry & $4.81 \times 10^{6}$ & - & $\begin{array}{l}4.0-4 \cdot 4 \\
\left(\mathrm{Me}_{3} \mathrm{~N}\right)\end{array}$ & $h, j, k, h, w$ & [29] \\
\hline$"$ & $"$ & & $"$ & $\begin{array}{l}\text { Slurried wich } 2 \text { units } \\
\text { of water }\end{array}$ & $4.81 \times 10^{6}$ & - & ${ }_{\left(\text {Me }_{3} \mathrm{~N}\right)}^{3.2}$ & & \\
\hline $\begin{array}{l}\text { Permutit } \\
\text { Deacidite }\end{array}$ & $\mathrm{OH}^{-}$ & 7.64 & $x$-ray machine $\left(1,10^{6}\right)$ & Air-dry & $.1 \times 10^{9}$ & 3 & - & $a, d, B, 1, k$ & [37] \\
\hline$A V-16$ & $\mathrm{OH}^{-}$ & 7.5 & ${ }^{60}$ Co r-radiations & Vacuua dry & $3 \times 10^{8}$ & - & 9 & $h, k$ & [1] Table 70 \\
\hline$"$ & $\mathrm{OH}^{-}$ & 7.5 & $"$ & Alr-dry & $5 \times 10^{7}$ & - & 4.5 & k & (1) Table 70 \\
\hline$"$ & $\mathrm{OH}^{-}$ & 7.5 & $"$ & A1r-dry & $5 \times 10^{7}$ & - & 2.0 & $k$ & $"$ \\
\hline$"$ & $\mathrm{OH}^{-}$ & 8.08 & Electron accelerator & Vacuus-dry & $3 \times 10^{8}$ & - & 0.1 & k & $"$ \\
\hline " & $\mathrm{OH}^{-}$ & 8.08 & " & Vacuuar-dry & $3 \times 10^{8}$ & - & 11 & k & $"$ \\
\hline$"$ & $\mathrm{OH}^{-}$ & 8.08 & " & Alr-dry & $2 \times 10^{9}$ & - & 1.2 & k & $"$ \\
\hline$"$ & $\mathrm{OH}^{-}$ & 8.08 & $"$ & $A 1 r-d z y$ & $2 \times 10^{9}$ & - & 0.5 & k & $"$ \\
\hline$A V-17$ & $\mathrm{OH}^{-}$ & 3.45 & $\begin{array}{l}\text { Electrog, accelerator } \\
\left(4.9 \times 10^{8}\right)\end{array}$ & In distilled water & $2.9 \times 10^{8}$ & - & 1.8 & - & [1] Table 77 \\
\hline$"$ & $\mathrm{cl}^{-}$ & 3.45 & $"$ & n & $2.9 \times 10^{8}$ & - & 2.3 & k & $"$ \\
\hline " & $\mathrm{NO}_{3}^{-}$ & 3.45 & $"$ & $"$ & $2.9 \times 10^{8}$ & - & 1.7 & $k$ & " \\
\hline " & $\mathrm{CrO}_{4}{ }^{2}$ & 3.45 & $"$ & $"$ & $2.9 \times 10^{8}$ & - & 2.4 & k & $"$ \\
\hline " & $\mathrm{CrO}_{4}^{2}$ & 3.45 & $"$ & $"$ & $2.9 \times 10^{8}$ & - & 3.6 & $k$ & $"$ \\
\hline$"$ & $\mathrm{PO}_{4}^{3}$ & 3.45 & " & " & $2.9 \times 10^{8}$ & - & 2.6 & $k$ & $"$ \\
\hline$"$ & $\mathrm{OH}^{-}$ & 3.5 & $"$ & " & $1.1 \times 10^{9}$ & - & 4.2 & - & " \\
\hline$"$ & $\mathrm{OH}^{-}$ & 3.6 & Reactor $\left(1.1 \times 10^{7}\right)$ & " & $2.5 \times 10^{8}$ & - & 32 & k & $"$ \\
\hline$"$ & $\mathrm{OH}^{-}$ & 3.6 & $"$ & $"$ & $2.5 \times 10^{8}$ & - & 24 & k & " \\
\hline " & $\mathrm{NO}_{3}^{-}, \mathrm{OH}^{-}$ & 4.3 & $\begin{array}{l}\text { Electron accele rator } \\
\left(3.6 \text { to } 5.0 \times 10^{8}\right)\end{array}$ & $"$ & $10^{9}$ & - & 2.6 & - & $"$ \\
\hline
\end{tabular}



TABLE IV (Cont'd)

\begin{tabular}{|c|c|c|c|c|c|c|c|c|c|}
\hline $\begin{array}{c}\text { RESIN } \\
\text { (Trade Nane) } \\
\end{array}$ & IONIC FORM & $\begin{array}{l}\text { EXCHANGE } \\
\text { CAPACITY } \\
\text { (meq/g) } \\
\end{array}$ & $\begin{array}{l}\text { RADIATION SOURCE } \\
(\mathrm{Rad} / \mathrm{Hr} .)\end{array}$ & $\begin{array}{l}\text { IRRADIATION } \\
\text { CONDITION(S) }\end{array}$ & $\begin{array}{l}\text { INTEGRAL } \\
\text { DOSE } \\
\text { (RAD) } \\
\end{array}$ & $\begin{array}{c}\text { LOSS OF } \\
\text { EXCHANGE } \\
\text { CAPACITY } \\
(y) \\
\end{array}$ & G VALUE & $\begin{array}{c}\text { OTHER } \\
\text { PARAMETERS } \\
\text { MEASURED } \\
\end{array}$ & $\begin{array}{l}\text { REFERENCE } \\
\text { NUMBER } \\
\text { AND NOTES } \\
\end{array}$ \\
\hline $\mathrm{AN}-25 \mathrm{Ax} 6$ & $\mathrm{OH}^{-}$ & 3.56 & Accelerated electrons & In distilled water & $1.44 \times 10^{9}$ & 0 & - & $b, e, h, k$ & {$[30]$} \\
\hline$A N-25 A \times 6$ & $\mathrm{OH}^{-}$ & 3.56 & $"$ & $"$ & $2.40 \times 10^{9}$ & 5.6 & - & - & $"$ \\
\hline EDE-1OP & $\mathrm{Cl}^{-}$ & 5.04 & $\begin{array}{l}{ }^{60} \mathrm{Co} Y \text {-radiation } \\
\left(6.6 \times 10^{5}\right)\end{array}$ & In water & $1.2 \times 10^{8}$ & - & 5.5 & - & [1] Table 77 \\
\hline EDE-10 & $\mathrm{OH}^{-}$ & 9.75 & ${ }^{60} \mathrm{Co} y$-radiations & Air-dry & $5 \times 10^{7}$ & - & 21 & $k$ & (1) Table 70 \\
\hline$"$ & $\mathrm{OH}^{-}$ & 7.23 & Electron accelerator & Vacuum-dry & $3 \times 10^{8}$ & - & 0.1 & k & [1] Table 70 \\
\hline$"$ & $\mathrm{OH}^{-}$ & 7.23 & $"$ & $A 1 r-d r y$ & $2 \times 10^{9}$ & - & 0.7 & $k$ & $"$ \\
\hline$"$ & $\mathrm{OH}^{-}$ & 9.75 & ${ }^{60} \mathrm{Co} \gamma$-radiations & Vacuum-dry & $3 \times 10^{8}$ & - & 14 & k & $"$ \\
\hline$"$ & $\mathrm{OH}^{-}$ & 9.75 & $"$ & Alr-dry & $5 \times 10^{7}$ & - & 26 & k & $"$ \\
\hline$"$ & $\mathrm{OH}^{-}$ & 7.23 & Electron Accelerator & Vacuum-dry & $3 \times 10^{8}$ & - & 12 & $k$ & $"$ \\
\hline VP-1 & $\mathrm{OH}^{-}$ & 7.6 & Electron Accelerat or & In distilled water & $3.4 \times 10^{9}$ & - & 0.1 & - & [1] Table 77 \\
\hline $\begin{array}{l}\text { Anton Exchanger } \\
\text { (not identified } \\
\text { by Trade Name) }\end{array}$ & $\mathrm{OH}^{-}$ & - & $\begin{array}{l}\text { y-radiation (spent } \\
\text { reactor fue } 1) \\
\left(2.6 \times 10^{6}\right)\end{array}$ & Covered with water & $\begin{array}{l}\text { Up to } \\
2 \times 10^{8}\end{array}$ & 22 to 30 & - & $b, d, i, k, m$ & $\begin{array}{l}\text { [14] Mixed } \\
\text { bed and par- } \\
\text { titioned } \\
\text { anion ex- } \\
\text { changer used }\end{array}$ \\
\hline
\end{tabular}

(a) welght change, (b) swelling/shrinkage or volume change, (c) water content, (d) solubility, (e) oxidizability, (f) gas evolution, (g) thermal stability, (h) products of decomposition, (1) formation of tontc species such as $\mathrm{so}_{4}^{-}, \mathrm{H}^{+}, \mathrm{Cl}^{-}$, etc., (j) use of scavenger, (k) exchange capacity measurements other than TEC, (1) at tempt to propose mechanist(s), (m) study of water soluble products, ( $n$ ) microscopic examination of resin beads. 
IV. GENERALIZATIONS ON THE EFFECTS OF RADIATION ON ION EXCHANGE RESINS

In many instances, the effects of intense radiation exposures of synthetic organic ion exchangers resemble similar effects observed on organic high polymers. However, the ion exchange resins are distinctly different in their macromolecular structure due to the presence of functional groups and the desired features of porosity and elasticity in the structure of resinous exchangers. In addition, the process applications of ion exchangers always involve other materials incorporated in the resin beads and the ion exchanger beds. This everpresent influence of foreign materials in the environment of ion exchangers and the distinct macromolecular structural features makes the overall radiation chemical changes in synthetic organic ion exchange resins extremely complex. On exposure to ionizing radiations, macromolecular structures undergo molecular changes such as bond breakages, bond formation, oxidation, and gas product formation. Of these, the reactions of particular significance are:

(i) the scission of chemical bonds of the main chain of the polymertc structure accompanied by a change in the molecular weight (degradation)

(ii) the changes in chemical bonds maintaining the tridimensional structure of the matrix (cross-linkage).

These reactions result in the formation of terminal or residual macroradicals, which in turn can enter into a variety of chemical transformations. Even though the radiation chemical yields in high polymers are relatively low, it is well known that the effects of radiation on the physical and chemical properties of organic polymers can be significant. In synthetic organic ion exchangers, these effects are enhanced by the relative ease of cleavage of the functional groups and secondary effects due to the products of radiation in the ion exchanger's 
vicinity as well as by the chemical effects such as oxidation, hydrolysis and double decomposition.

The changes in the molecular structure of organic polymers leads to changes in their density, molecular weight, elasticity, porosity, solubility, thermal stability and other mechanical as well as electrical properties. Similar changes in ion exchangers are compounded by both direct radiation effects on the ion exchangers macromolecules and the secondary effects mentioned above. Little is known about the mechanisms of the various radiation chemical effects on ion exchange resins. This is primarily due to the complexity of these matrices and the limited efforts spent so far in the detailed study of these radiation chemical changes. Although most of the presently known efforts at explaining the mechanisms of these reactions are extensions of knowledge gained in the study of radiation effects on high polymers and aqueous systems, there have been several experimental studies directed at elucidating the modifications in the properties of ion exchangers which are of importance in process applications. The present knowledge on these changes in the properties of synthetic organic resins can be summarized in the form of a set of qualitative and semiquantitative generalizations ("rules of thumb"). These generalizations listed below are derived from various experimental studies surveyed during this study. Additional discussions of these rules and known exceptions are considered in the following section of this report.

In listing these generalizations, it should be pointed out that while there are agreements in several areas among experimental findings of several workers, there exist both differences of opinions and demonstrated exceptions to these rules. The qualitative and semi-quantitative generalizations are: 
1. Radiation chemical changes in Ion exchange resins are a direct function of the total dose absorbed by the resin.

2. Most anion exchange resins show considerable degradation when the total absorbed dose increases above $10^{7}$ to $10^{8}$ rads, while most cation exchangers show significant attrition only above $10^{8}$ rads.

3. In most ion exchange resins, an absorbed dose of $10^{9}$ rads or higher results in sufficient damage to make the material unserviceable.

4. There are two well recognized forms of radiation effects on ion exchange systems - one is the direct action of radiation on the ion exchanger matrix while the other is the indirect action of radiolytic products of the materials in the environment of the resin.

5. The primary effects of radiation on fon exchange resins are degradation and cross-linking of the macromolecular structure along with the scission of lon exchange functional groups.

6. The radiation chemical ylelds (G value) for radicals, ions, gaseous and other decomposition products are not constants. They vary within the same resin depending on factors such as temperature, constituents of the matrix of the resin and the nature of the medium surrounding the resin.

7. Like high polymers, the free radicals formed during radiolytic decomposition of Ion exchangers may be stable for extended periods at ordinary temperatures.

8. The free radical concentration in fon exchange resins belng irradiated Increase steadily and tend to reach a limiting concentration. This limiting concentration of free radicals is reached at integral doses of $10^{8}$ to $10^{9}$ rads In most resins. 
9. The exchange capacity of Ion exchange resins, in general, decreases with increasing radiation dose.

10. In cation exchangers, there is initially an increase in functional groups capable of exchange as a result of Irradiation in the presence of air or moisture. These are phenolic and carboxyl groups produced as a result of oxidation.

11. The initial increase in exchange sites tends to increase the total exchange capacity (TEC) of the resin. However, the accompanying scission of existing exchange groups often results in a net decrease in TEC values.

12. The overall radiation chemlcal change in ion exchangers is drastically different for a given resin Irradiated in the presence of water or aqueous solutions, when compared with simllar irradiations in the dry state. Some of the important properties (especially TEC values) of Ion exchangers important to process applications change by a factor of 2 to 5 during Irradiation In aqueous media.

13. Radiation resistance of Ion exchangers increases with increase in their cross-1inkage.

14. Radiation chemical changes producing cross-1inkages are more dominant in ion exchange resins of lower cross-1inkage.

15. Although anton exchange resins in general are more susceptible to radiation damage compared to cation exchangers, there are spectal formilations of anton exchangers (pyridine-dertved) which are significantly superior in their radiation resistance than most cation exchangers.

16. While the physical, chemical and mechantcal properties of polymertzation type resins are generally superior to those of polycondensation type resins, the polycondensation type resins are superior in their radiation resistance. 
17. Among polycondensation type resins, the exchangers with structural aromatic groups are more radiation resistant when compared with purely aliphatic resins. This protective effect of aiomatic groups is found in all organic systems and it is generally true in synthetic resins.

18. In general, the salt forms of ion exchange resins are more resistant to radiation than the $\mathrm{H}^{+}$or $\mathrm{OH}^{-}$forms.

19. Organic resins show variations in the protective effect (radiation protection) depending on the salt forms of the resins. The protective effects are significantly higher when ions of variable valences are involved.

20. The radiation stability of polymeric styrene-divinylbenzene resins, when irradiated in water in the $\mathrm{H}^{+}$or $\mathrm{OH}^{-}$forms show the following order of stability:

Pyridine anion exchangers > nuclear sulfonic and carboxyl cation exchangers > weakly basic anion exchangers of the primary, secondary and tertiary type > strongly basic anion exchangers of quarternary ammonium base type.

21. Strong (acid/base) monofunctional ion exchange resins, in general, are more resistant to radlation damage than weak (acid/base) resins and polyfunctional resins.

22. The radiation protection of ion exchange systems can be improved through the presence of scavengers such as methanol, ethanol, cysteamine, cysteine, etc., although their potential process applications have not been reported.

23. Under identical conditions, when radiation sources of low LET values ( $\gamma$-rays, $x$-rays, fast electrons and $\beta$-particles) are used, there are no apparent differences between the radiation effects on resins, whether the source is external or internal. 
24. There are some recognizable differences between the radiation chemical effects on ion exchange resins of high and low LET radiation. This is particularly true between internal irradiation with heavy particles (recoll protons and a-particles) of high LET values and with internal or external 1rradiations with particles of low LET values.

25. Among synthetic organic cation exchangers, the resins with carboxylic functional groups are least resistant to radiation and those with phosphate functional groups are most resistant.

26. During irradiation in aqueous media, the swelling tendency of ion exchange resins shows an inttial increase (up to $\sim 10^{6}$ rads) followed by a decrease due to the molsture absorption capacity steadily decreasing.

27. The swelling capacity of a resin is a function of the medium in which the resin is irradiated.

28. The polycondensation type resins show an extended duration for the increase in swelling ability compared to polymerization type resins. Both types continue to show decreased swelling as the dose reaches $10^{7}$ rads or higher.

29. Radiation effects on organtc ion exchangers result in column changes of the resin matrix. The resins change from their original shades to amber, brown, dark red and black with Increased doses.

30. As a consequence of radiation damage, the particles of resins (beads) become gouged, pitted, cracked and at times solubilized when the integral dose exceeds $10^{8}-10^{9}$ rads.

31. The effluents from Ion exchange columns being exposed to lonizing radiation show measurable changes (decrease). In $\mathrm{pH}$. At an integral dose of $10^{8}$ rads, the effluent from anion exchangers also begin to show a darkening effect 
due to solubilization. For cation exchangers, this attrition starts at higher doses.

32. Prolonged exposure of ion exchange resins to radiation in flowing (dynamic) systems causes more drastic changes in their physical and chemical properties than ion exchange resins irradiated in static systems.

33. Irradiated resins are less resistant to degradation by thermal effects.

34. Gaseous products resulting from the action of lonizing radiation on Ion exchange resins primarily consist of hydrogen. Other gaseous products of resin decomposition include $\mathrm{CO}_{2}, \mathrm{CO}, \mathrm{N}_{2} \mathrm{O}$, NO and $\mathrm{SO}_{2}$.

35. The radiation chemical yield of $\mathrm{H}_{2}, \mathrm{CO}_{2}$ and $\mathrm{CO}$ in general show a linear dependency on dose.

36. Gaseous products in ion exchange columns exposed to radiation are llable to produce gas blocks and channelling, the results of which can be more catastrophic than the radiation degradation of the resin matrix.

37. Synthetic organic ion exchangers which are not true compounds are significantly less resistant to radiation damage when compared with inorganic ion exchangers, which are well defined chemical species. However, there exist other serious limitations to the use of Inorganic ion exchangers in radiochemical processing.

These generalizations are valuable in qualitatively evaluating the radiation chemical effects on synthetic organic fon exchangers. However, it should be recognized that the processes involved are too complex to be presented as simple rules, which are universally applicable. Some discussions of important phenomenological changes, proposed mechanisms and recognized limitations to the above mentioned general rules are considered in the following section. 


\section{RADIATION EFFECTS ON ION EXCHANGE RESINS}

Ionizing radiation doses of ten to one hundred megarads significantly alters the properties of synthetic organic ion exchangers. Anion exchangers, in general, exhibit less resistance to radiation damage when compared with cation exchangers. The radiation chemical effects on ion exchange resins are dependent on several factors related to the resin itself. These include the chemical composition, the ionic form in which they are exposed to radiation, the moisture content and the degree of crosslinkage of the macromolecular structure. In addition, many factors related to the radiation sources and the environment of the resins during irradiation control the overall radiation chemical change that of the ion exchange resins experience.

\section{V.1 Polymerization Type Cation Exchange Resins}

Since many of the commercially important cation exchangers are polymerized nuclear sulfonic type, many of the reported experimental studies have used this type of resin. It has been satisfactorily demonstrated that the radiation chemical stability of polymerization type cation exchange resins is a function of the degree of cross-1inkage of their macromolecular structure. Several experimental studies have confirmed that the radiation stability of these resins increases with increased cross-1inkage. Early work by Wedemeyer (37) and Cathers (95) on styrene-divinylbenzene polymerization type Nalcite-HCR resins, as well as more recent studies by Semushin, et al. (9) on methacrylic aciddivinylbenzene copolymer $\mathrm{KB}-4$ and Riseleva et al. (41) on acenaphthalenedivinylbenzene $\mathrm{KU} 4$, are typical of the various studies reported, illustrating the relationship between radiation stability and cross-linkage. There have been several other investigations during the past two decades that have generally concluded that an increase in cross-linkage had beneficial effects on the radia- 
tion stability of synthetic organic catjon exchangers $(4,10,18,22,23,43$, $46,48,49,93)$.

One of the ways of characterizing the stability of ion exchangers is by measuring the change in total exchange capacity (TEC) as a function of irradiation. Many commercially important polynerization type cation exchangers have been examined using these procedures. Resins with crosslinkage varying from 4 to $24 \%$ examined during various experimental studies have confirmed the stabilizing influence of cross-linkage both in the starting material and the cross-1inkage produced during the course of irradiation. While resins with low cross-linkage show a higher radiation damage initially, the subsequent changes in the TEC are more or less identical. This may be due to the initial processes involving cross-linkage and rupture of $-\mathrm{SO}_{3} \mathrm{H}$ groups resulting in resins of somewhat similar radiation chemical properties during the initial stages of irradiation.

Unlike the generally held view, Tulupov et al. (43) reports that an increase in cross-1inkage (DVB content) of the nuclear sulfonic resin, $\mathrm{KU}-2$, from $8 \%$ to $24 \%$ has no significant effect on the radiation stability as measured by the TEC. Detailed results of this study are tabulated in Table III.

There are very few experimental studies on radiation chemical effects on ion exchange resins that can be justifiably compared to generate quantitative data typical of a class of synthetic resins. Figure 1 is a comparison of three independent experimental studies $(23,43,49)$ using nuclear sulfonic styrene-DVB polymeric resins. The details of the experimental conditions and a comparison of the change in TEC at a dose of about $5 \times 10^{8}$ rads are summarized in Table $v$. The significant differences in the rate of change of TEC with dose in these three studies cannot be adequately explained by the minor changes in the experi- 


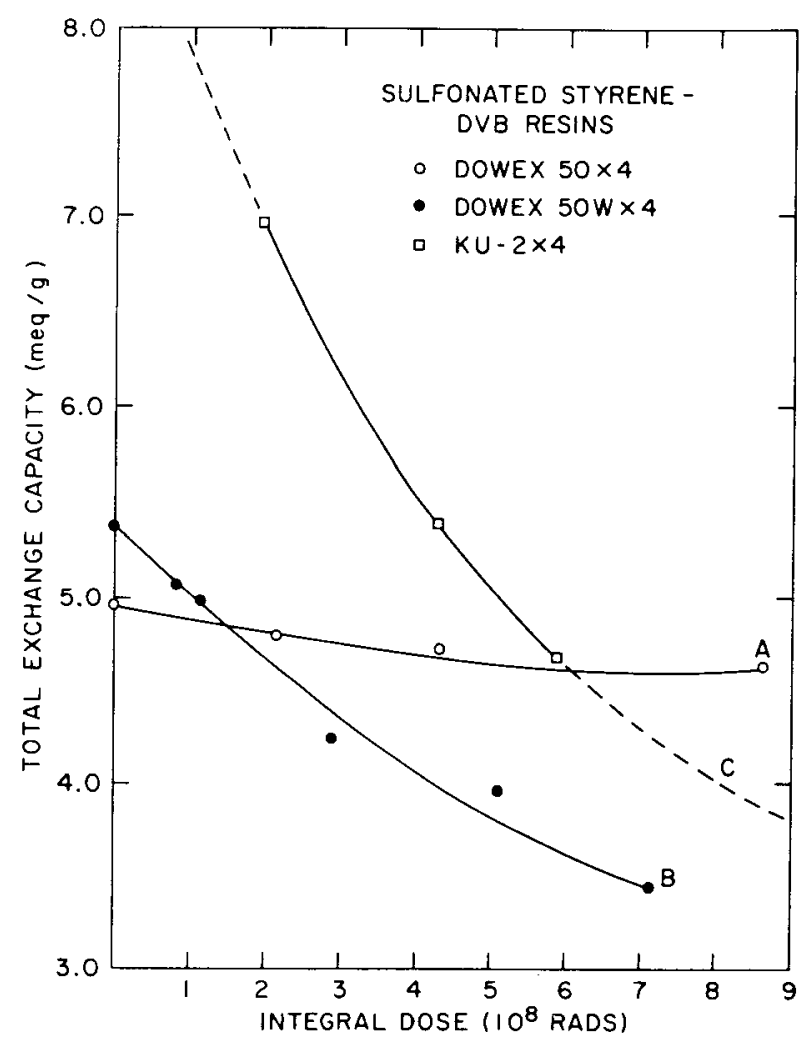

Figure 1. A comparison of the change in total exchange capacity (TEC) of $4 \%$ cross-Iinked styrene-DVB sulfonic acid resin $[23,43,49]$.

Table V

COMPARIS ON OF THE RESULTS OF THREE EXPERIMENTAL STUDIES

ON 4\% CROSS-LINKED STYRENE-DVB RES IN

\begin{tabular}{|c|c|c|c|c|c|}
\hline Resin & $\begin{array}{l}\text { Radiation } \\
\text { Source }{ }^{\star}\end{array}$ & $\begin{array}{c}\text { Water } \\
\text { Content (\%) } \\
\end{array}$ & $\begin{array}{c}\text { Initial } \\
\text { TEC } \\
\end{array}$ & $\begin{array}{l}\% \text { Chan ge } \\
\text { in rectt }\end{array}$ & Ref. \\
\hline Dowex $50 \times 4$ & $\begin{array}{l}\text { X-rays } \\
\left(1.44 \times 10^{7}\right)\end{array}$ & 28 & 4.95 & 42 & (44) \\
\hline Dowex 50 w $\times 4$ & $\begin{array}{l}\gamma \text {-rays } \\
\left(1.25 \times 10^{6}\right)\end{array}$ & 66 & 5.35 & 66 & (23) \\
\hline $\mathrm{KU}-2 \times 4$ & $\begin{array}{l}\text { Y-rays } \\
\left(8.46 \times 10^{6}\right)\end{array}$ & 70 & 10.0 & 50 & 43 \\
\hline
\end{tabular}

*The number in the paranthesis indicates the radiation dose rate in $\mathrm{rads} / \mathrm{hr}$.

HTEC is the total exchange capacity of the resin. 
mental parameters of these three investigations. This type of difference in the reported results of different experimental studies on similar types of resin are not uncommon.

A factor that may be responsible for the differences in the results of various experimental studies is the difference in the constituents of the resin. Such differences can be due to various impurities that are commonly present in the starting materials used in the polymerization of the resin itself. Wiley et al. (46) reports an interesting study on the radiation stability of sulfonated styrene-divinylbenzene resins with various isomers of divinylbenzene and mixtures of the isomers of this cross-linking agent. Wiley, et al. have found that there are measurable differences in the degree of radiation chemical decomposition of commercial resins compared to various laboratory preparations of the same composition, the latter being more radiation resistant. Further, it is reported that the variations in the use of pure para- or meta- substitution products of DVB in resin preparations did not result in any significant differences in the radiation stability of the product. In a systematic investigation of the constituents of commercial DVJB, using vapor-phase chromatographic separations, Wiley and Associates ( 91 ) found that the commercial DVB contained eight or ten different compounds and their ratio varied in DVB from different commerical sources. The authors point out that it may be the ethylvinylbenzene impurity in DVB used in some of the preparations which enhances its radiation stability (46). It is also suggested (92) that the structural factors which presumably create differences in the rates of sulfonation for $p$ and $m-$ substituted DVB should be operative in the desulfonation during irradiation and the reactions induced under the influence of radiation. 
An important parameter that is often used as a measure of radiation chemical effects is the $G$ value. The degree of cross-linkage of the resin and its relationship to $G$ value has been reported during several investigations. The results of one such study (49) are illustrated in Figure 2. The data presented are in terms of energy required (in e.v.) per $-\mathrm{SO}_{3} \mathrm{H}$ group released during the irradiation of the nuclear sulfonic resins of various cross-linkage. In 4 and 8 percent cross-1inked Dowex 50 resins, there is an initial sharp increase in the energy required (or a sharp decrease in the $G$ value) for the removal of $-\mathrm{SO}_{3} \mathrm{H}$ groups, whereas in resins with higher cross-linkage such a deflection in the slope of the curve is not apparent. The change in G value with dose is explained by the changes in the resin matrix. Prior to irradiation, the $-\mathrm{SO}_{3} \mathrm{H}$ groups are uniformly distributed in the resin matrix. During early stages of irradiation, the externally located functional groups would be readily accessible for direct interaction of radiation or radiation produced species. However, as the irradiation progresses, loss of the internally located $-\mathrm{SO}_{3} \mathrm{H}$ groups, which are less susceptable to direct or indirect action of radiation becomes important and therefore more and more energy has to be expended per dissociation of the remaining $-\mathrm{SO}_{3} \mathrm{H}$ groups .

Another property of ion exchange resins that changes with cross-1inkage is the swelling ability of the matrix. Swelling of the resin is caused by the expansion of the structure as a result of water (or other liquid) molecules penetrating the matrix. This effect is a maximum for low cross-linkage resin because of the flexibility of the macromolecular structure. Water is also associated with the hydrophylic exchange groups, which also contribute to swelling.

It is generally believed that the swelling ability of cation exchange resins decreases as a result of radiation exposure. As the irradiation of resin 


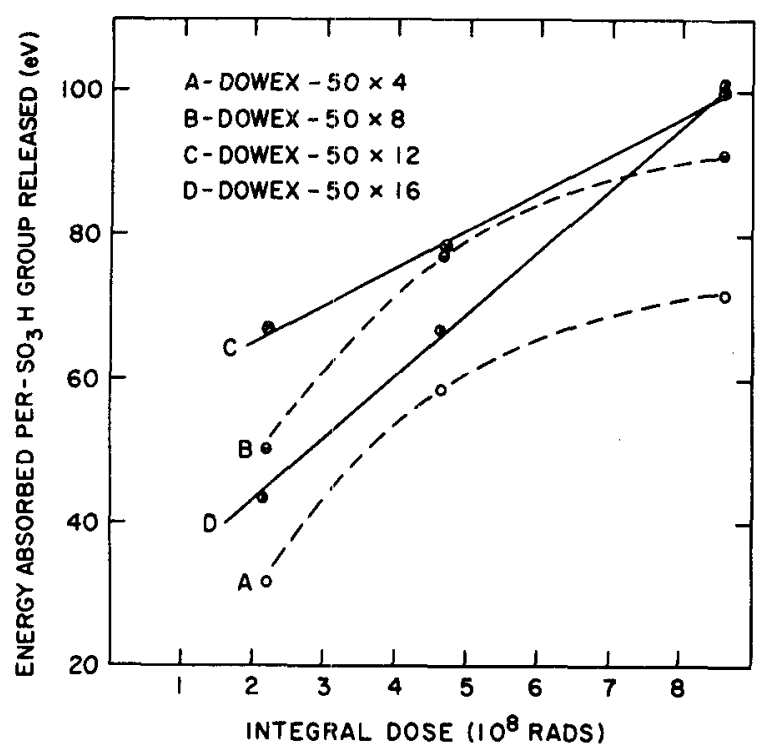

Figure 2. Changes in radiation chemical yield (expressed as $\mathrm{eV} / \mathrm{SO}_{3} \mathrm{H}$ group released) as a function of cross-linkage and dose [49]. 
is continued, the cross-linkage increases and the functional groups are ruptured resulting in a decrease in the water content of the resin matrix. A typical study (51) of the change in swelling of the resin as a function of total dose and dose rate is illustrated in Figure 3. The sulfonic cation exchange resin KU-2 shows significant changes in its swelling capacity up to a total dose of 4 $x 10^{9}$ rads and a complete breakdown beyond $10^{10}$ rads.

Utley (49) has shown that for various cross-1inkage of Dowex-50 resin with a moisture level of $\backsim 25 \%$, there is a consistent decrease in water absorption capacity, when exposed to x-rays. However, Smith, et al. (22) have pointed out that the moisture content of Dowex $50 \times 12$ increases steadily up to $\Omega 2.8 \times 10^{8}$ rads, which they believe is due to a decrease in the cross-linkage of this resin during irradiation. Similar increases in moisture holding capacity of Dowex-50 have been reported by Bauman (14) and by Kazanjian et al. (23, 25). One further observation by Kazanjian et al. (25) is that the moisture content of Dowex-50 W begin to decrease when the radiation dose increases above $\sim 4 \times 10^{8}$ rads. Similar observations have been reported by Dornow et al. $(11,96)$ for the cation exchanger AG 50W X 8 and by Kiseleva et al. (52) for KU-2 sulfonic acid resin. During irradiation in water, it is observed that the swelling capacity of $\mathrm{KU}-2$ resin increased with dose of $\backsim 8 \times 10^{7}$ rads, but with higher doses the swelling decreased. In acidic solutions, however, the decrease in swelling capacity at higher doses was not as significant as irradiations in pure water $(52)$.

Cation exchangers of the styrene-DVB type undergoes weight losses during irradiation. This change in weight is attributed to the attrition of the macrostructure due to rupture of functional groups and evolution of gaseous products. The weight change of resin is both dose and dose-rate dependent (51). Figure 4 is an illustration of the percent loss or weight of the sulfonated 


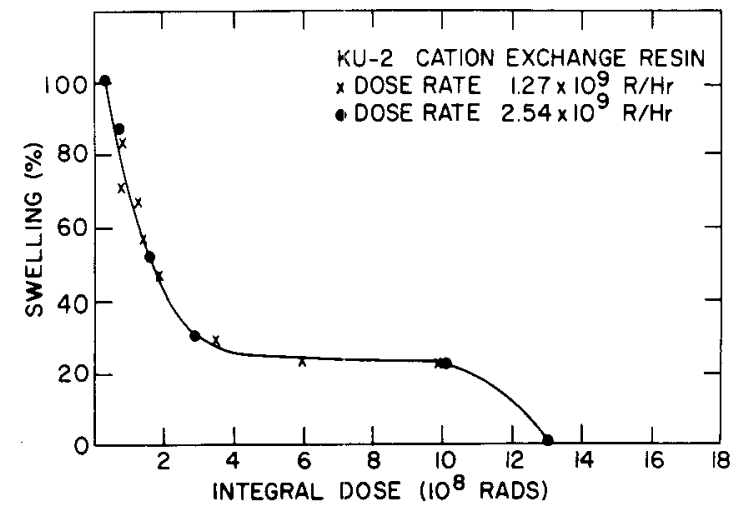

Figure 3. Change in swelling capacity of styrene DVB copolymer with dose and dose-rates [51].

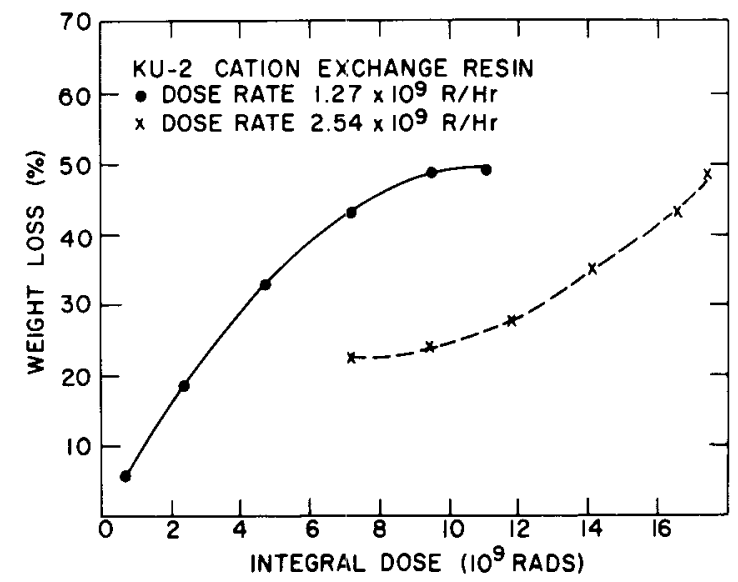

Figure 4. Weight loss of styrene-DVB sulfonic resin as a function of dose at two different dose rates [51]. 
styrene-DVB copolymer as a function of dose (51). The data illustrating the loss of weight at higher dose rate shows increased attrition rate and higher rate of weight 1oss. In a more recent study, Pesek and Spurny (97) examining a variety of strongly acidic cation exchange resins, have shown that both the change in exchange capacity and weight losses depend significantly on the degree of cross-linkage and porosity of the resin.

As pointed out earlier, radiation chemical effects are influenced by the moisture content of the resin. An illustration of the loss of exchange capacity (based on $-\mathrm{SO}_{3} \mathrm{H}$ groups) as function of water content of the resin is presented in Figure 5. This data from Kiseleva, et al. (38) shows that the radiation chemical effects are significantly influenced by the moisture content of the resin until the sorbent-water ratio reaches a value of about $1: 1$. Beyond this point, the water content does not seem to have any significant influence on the rates of reactions. The dependence of total dose on the loss of exchange capacity are also illustrated in Figure 5.

A study of the interaction of sulfonic acid ion exchange resins with tritiated water during $\alpha$-irradiation has shed some light on the degree of interaction between the radicals produced in the resin structure and those resulting from the radiolysis of water molecules. Mohorcic et al. (33) found that during irradiation of ion exchange resins in tritiated water, a significant amount of hydrogen $\left({ }^{3} H\right)$ from water gets irreversibly bound in the resin matrix. The data from this study (33) included in Table III and illustrated in Figure 6 shows the variations in the bound tritium in the resin phase as a function of dose at various moisture content. In the reported results, the authors chose to ignore isotope eifects and conclude that the reactions of tritium radical with 


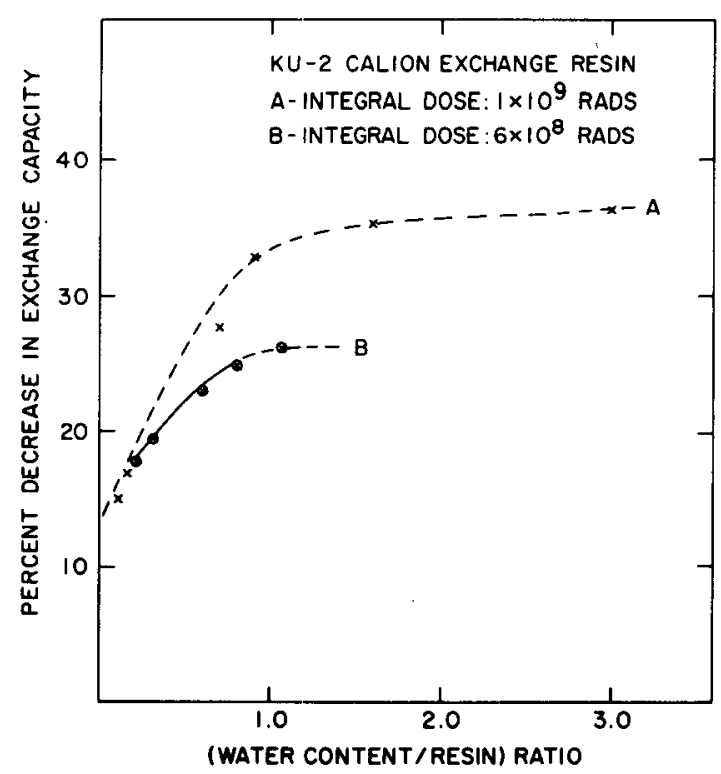

Figure 5. Loss of exchange capacity (based on $\mathrm{SO}_{3} \mathrm{H}$ groups) as a function of water content [38].

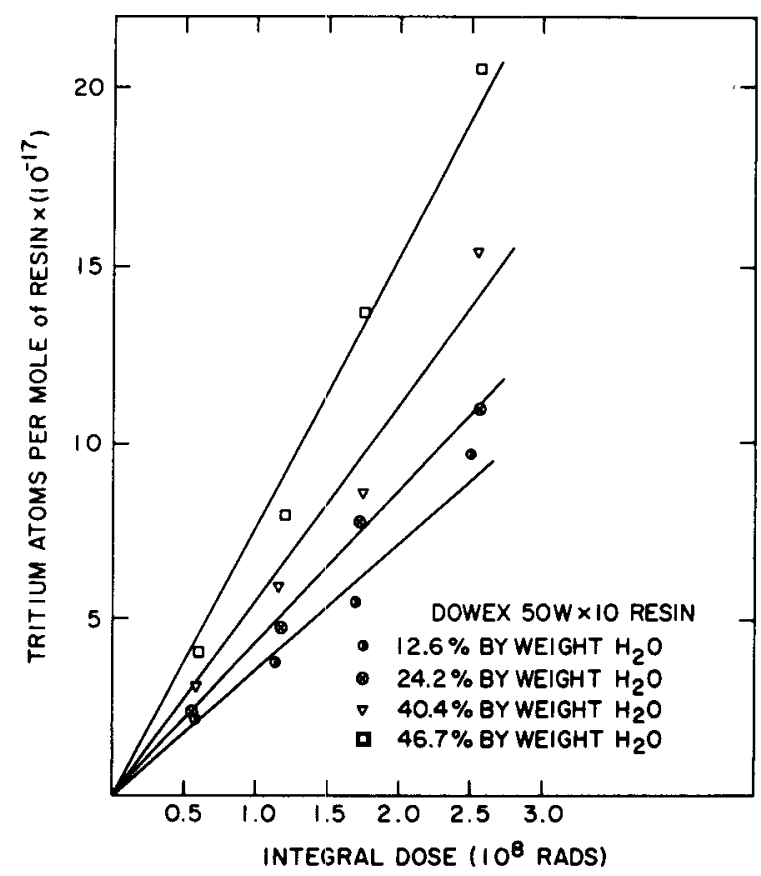

Figure 6. Irreversible bonding of tritfum from water in the cation exchanger matrix during irradiation [33]. 
carbon atoms from which the $-\mathrm{SO}_{3} \mathrm{H}$ group has ruptured could be the most important mechanism by which the tritium gets incorporated into the resin matrix.

Several experimental studies, under a variety of conditions, have demonstrated that the presence of air, oxygen and moisture accelerates radiation decomposition of cation exchangers. A parallel effect identified in the polymerization type cation exchange resins is the formation of new functional groups that act as new exchange sites for cations. Through careful experimentation involving the study of dissociation constants of the acid radicals involved, these new functional groups formed during the irradiation of cation exchange resins have been identified as phenolic hydroxyl groups and carboxylic groups. The results of one of these studies $(51)$ is illustrated in Figure 7 . The formation of $-\mathrm{OH}$ and $-\mathrm{COOH}$ groups in the resin matrix increases the exchange capacity of the resin. However, the net change in TEC is a decrease because of the relatively increased loss of $-\mathrm{SO}_{3} \mathrm{H}$ functional groups which provides some of the sites for the formation of $-\mathrm{OH}$ and $-\mathrm{COOH}$ groups. Figure 7 shows the net change in exchange capacity as well as the changes in the exchange capacities due to $-\mathrm{SO}_{3} \mathrm{H},-\mathrm{OH}$ and $-\mathrm{COOH}$ functional groups. Riseleva, et al. ( 51$)$ have studies the formation of new functional groups at three different dose rates and only a few of these results are included in Figure 7 . Total exchange capacity for the irradiation at a dose rate of $1.27 \mathrm{rads} / \mathrm{hr}$ was calculated by summing up the reported values of the exchange capacities for the three functional groups. These authors, while studying the effect of different types of aqueous media on the formation of new functional groups, have demonstrated that the rate of formation of new $-\mathrm{OH}$ and $-\mathrm{COOH}$ groups are most pronounced in water, when compared with aqueous solutions of nitric, lactic and acetic acids (52). 


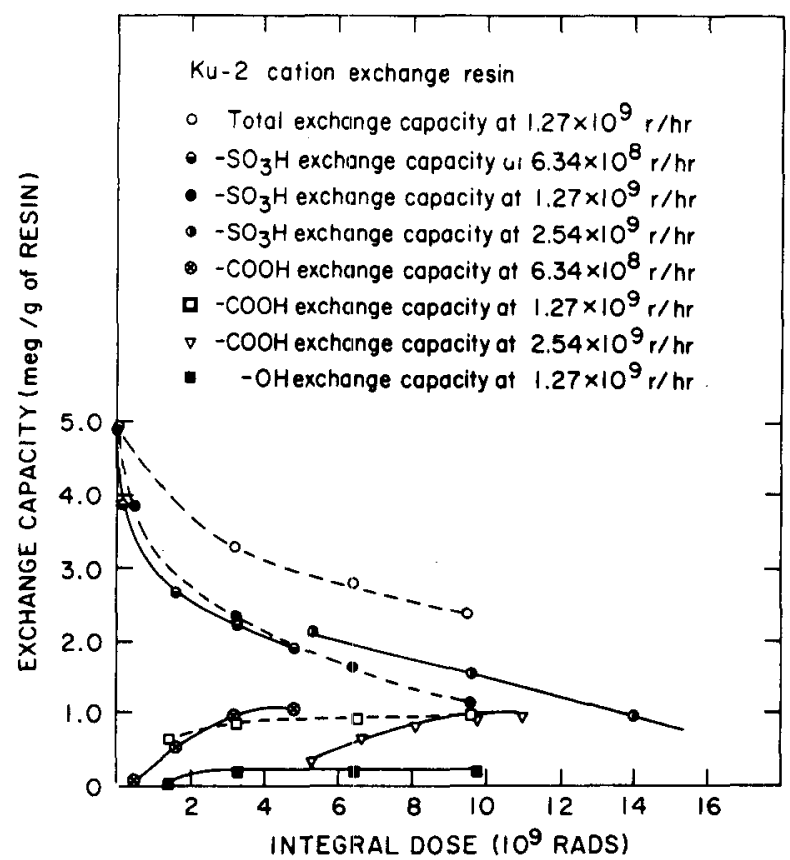

Figure 7. Variation in the TEC and exchange capacities with respect to individual functional groups during the irradiation of a sulfonlc polymerization type resin [51]. 


\section{V.2 Other Types of Cation Exchange Resins}

Cation exchange resins of the polycondensation type are more resistant to radiation than the polymerization type resins, although their chemical, mechanical and thermal properties are significantly poorer. Experimental studies using Dowex-30, Amber1ite IR-105, Duolite C-3, Zeolex, KU-1, KU-5, KU-32, KU-33, Catex FN, $F_{2} S, R F-8$ etc. have repeatedly confirmed this generalization $(1,6,12,34$, $39,40,54,58,94,95)$. However, there is a reported study by C'ostachel and Furnica (96), which does not support this generalization.

The change in properties such as the decrease in TEC, change in the swelling ability, appearance of new functional groups, weight loss and gas evolution are common to both types of cation exchange resins. The difference between the two types being in the rate of change of these properties with dose, which take place at a slower pace in the polycondensation type cation exchange resins.

Among the strong acid type cation exchange resins containing $C-S$ bonds, the chemically unstable phenol-sulfonic acid-formaldehyde resin is more stable to ionizing radiation in a variety of aqueous solutions than the chemically stable styrene-DVB-sulfonic resin (58).

Investigations of radiation stability of cation exchangers in relation to their functional groups have showed that the weakly acidic carboxylic acid resins containing $\mathrm{C}-\mathrm{C}$ bonds are more resistant to radiation than the strongly acidic resins containing $C-S, C-P$ or $C-0$ bonds $(6,12,58)$. A polycondensation resin based on salicylic acid and furfural has high resistance to radiation compared to several condensation products of sulfonic acid derivatives (6). Its exchange capacity, swelling and other characteristics are unchanged at radiation doses of $2 \times 10^{8}$ rads. Shigematsu and Oshio (12) confirms this generalization 
of the radiation stability of weakly basic cation exchangers. Among eight different types of cation exchangers exposed to a total dose of $\sim 4 \times 10^{7}$ rads, a carboxylic acid resin, Duolite CS101 showed an increase of $2 \%$ in TEC. All. the other cation exchangers in this study (12) showed varying losses of TEC ranging from 0.5 to $10.5 \%$ during the period.

\section{V.3 Radiation Protective Effect of Some Cation Exchangers}

The radiation protective effect of ion exchange resins primarily. result from the nature of their macromolecular structure. The protective effect of macromolecules such as naphthalene, anthracene, phenamthrene and hydroquinone in polycondensation type resins have been systematically studied $(40,54)$. The findings are in agreement with the established findings concerning the protective effect of aromatic compounds. However, there are no significant differences in radiation stabilities among the resins containing the above mentioned aromatic configurations $(40)$.

Another form of radiation protection effect is known to exist in salt forms of cation exchangers. In general, the salt forms of cation exchange resins are more radiation resistant than their $\mathrm{H}^{+}$-forms. Further examination of this phenomenon has repeatedly demonstrated that in aqueous solutions, certain ionic species attached to the resin provides a significant radiation protection effect $(9,10,38,42,56,60,61)$. This protection effect is exceptionally predominant in cation exchangers with ionic species of variable valence (10, 38). One unique exception reported (1) is that the radiation stability of the salt forms of polycondensation type cation exchange resin (even with reducible ionic species) is not significantly different from ițs $\mathrm{H}^{+}$-form.

Ichikawa et al. (10) reported on the effects of adsorbed species on radiation damage to Dowex $-50 \mathrm{~W}$ and Kiseleva, et al. (38) have done similar studies 
using $\mathrm{KU}-2$ cation exchange resin. When Dowex $-50 \mathrm{~W}$ resins of various ionic forms were exposed to similar doses of $\gamma$-radiation, the loss of exchange capacity varies from $\checkmark 1$ to $7 \%$ depending on the ionic form of the resin (10). Some of the results from this investigation and (38) are summarized in Table VI which illustrates the distinct protective effect by variable valence ionic species on sulfonic polymerization type resins. In addition, Ichikawa et al. (10) also report on the irradiation of Dowex-50 $\mathrm{W}$ in $\mathrm{Na}^{+}$and $\mathrm{K}^{+}, \mathrm{NH}_{4}^{+}, \mathrm{Ca}^{2+}, \mathrm{Mg}^{2+}$ and $\mathrm{Mn}^{2+}$ forms. None of these seem to have any significant effect on the radiolytic decomposition of the resin under similar conditions of irradiation. Similar findings were reported by Skorokhod, et al. (42) showing that there is no appreciable difference in the radiation damage to $\mathrm{KU}-2$ resin when irradiatied in the $\mathrm{H}^{+}$form or in $\mathrm{Li}^{+}, \mathrm{Na}^{+}$and $\mathrm{K}^{+}$forms.

Similar protective effects of $\mathrm{Cu}^{2+}$ ions in resin matrix have been observed for amphoteric resins (56) and for methacrylic acid-DVB copolymers (9).

\section{V.4 Anion Exchange Resins}

Radiation effects on anion exchange materials are less extensively studied compared to the study of similar effects on cation exchangers. Resins of the polymerization type incorporating quarternary ammonium groups -$\mathrm{C}_{6} \mathrm{H}_{4} \mathrm{CH}_{2} \mathrm{~N}^{+}\left(\mathrm{CH}_{3}\right)_{3} \mathrm{OH}--$ continue to be the most important anion exchanger used in the process industry. Several of this type of resin have been the subject of radiation chemical effect studies over the past two decades $(1,12,13,16,17$, $19,20,22,24,26,28,29,30,31,32,37,50,53,62,63,97)$. However, on 1y a few of these experimental studies offer any quantitative data of significance that are applicable to all polymerization type anion exchangers.

Radiation chemical changes in anion exchange resins are characterized by darkening of the color of resin, evolution of odorous free amines, gas evolu- 
Table VI

EFFECT OF IONIC FORMS OF CATION EXCHANGE RES INS

ON RADIAT ION STAB ILITY

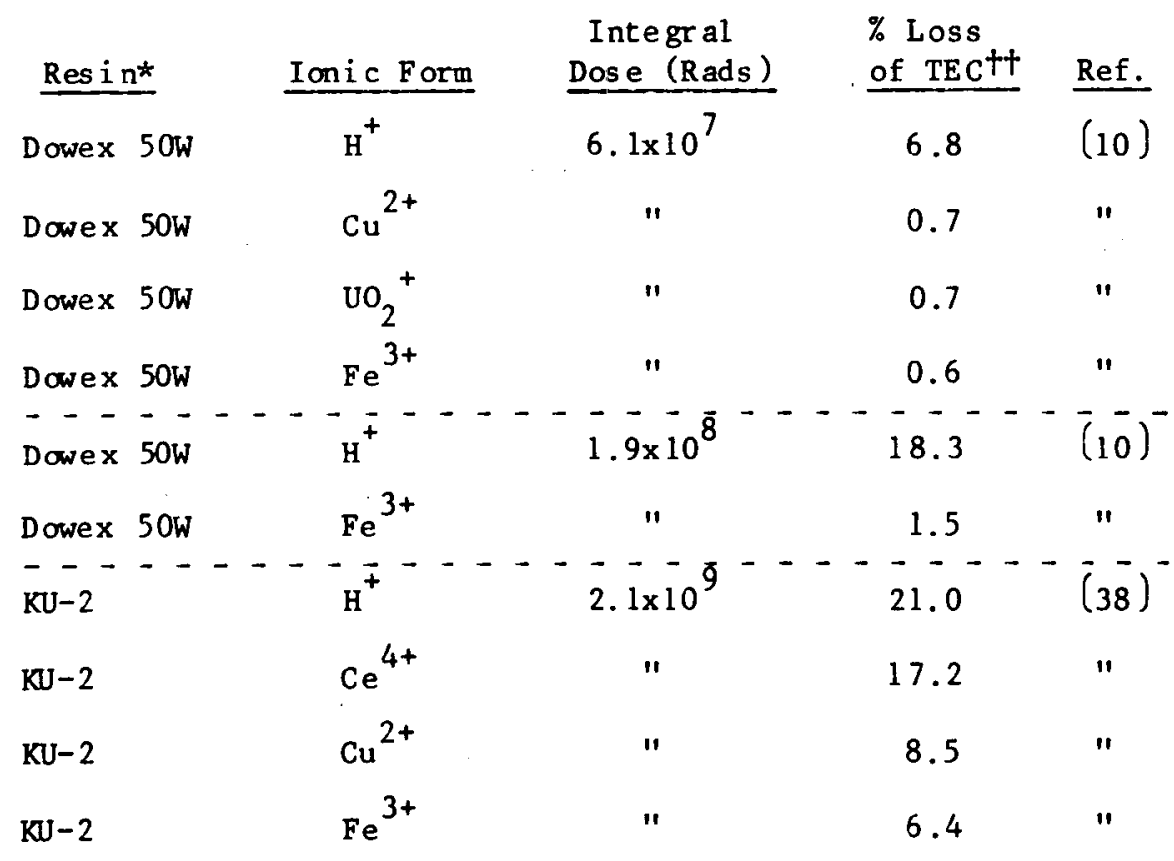

*Dowex $50 \mathrm{~W}$ resin was irradiated in wet conditions and $\mathrm{KU}-2$ resin in the absence of air.

HTEC is the total exchange capacity of the resin. 
tion, loss of exchange capacity, decrease in swelling capacity and degradation of the resin matrix. As a rule, the resistance of anion exchange resins to radiation does not vary very much with the basicity of the resin. This is in contrast to the high radiation stability of strong acid cation exchange resins compared to weakly acidic resins. Among various anion exchange resins, those containing aromatic systems with conjugated bonds in the functional groups are more resistant to radiation than all other types of synthetic organic ion exchangers. Examples of the radiation stabilities of this type of resin are further discussed in Section V.5 of this chapter.

Most of the polymerization type anion exchange resins are based on styrenedivinylbenzene copolymers. Even though this structure is known to be highly resistant to radiation damage, the anion exchangers containing quarternary, teritrary, secondary or primary amino groups begin to show marked attrition at doses of $\sim 10^{7}$ rads. Cation exchange resins of similar structural origin generally suffer little damage at $10^{7}$ rads of exposure. Again, in general, the attrition of anion exchangers are more extensive in the presence of water or aqueous electrolytes as compared to irradiations of resins in the airdry state.

The available experimental data on the radiation effects on anion exchangers indicate that a complex mechanism of radiation induced chemical changes takes place in strongly basic monofunctional anion exchangers. It is suggested $(1,20,28)$ that there may exist two types of exchange groups that differ in relative stability toward radiation chemical changes. These groups are believed to be the ones attached to the aromatic ring in ortho- and parapositions; the ortho groups being less stable than the para-substituents (20). There is adequate experimental evidence to suggest that strongly basic 
quarternary ammonium type resins undergo radiation decomposition primarily via deamination processes. This leads to the breakage of the $\mathrm{C}-\mathrm{N}$ bond connecting $\mathrm{N}\left(\mathrm{CH}_{3}\right)_{3}$ groups with the benzyl radicals $(16,17,28)$. Other competing reactions, in addition to $\mathrm{C}-\mathrm{N}$ bond rupture, have been suggested by Hall and Streat (28), Basinski et al. (16) and by Narebska, et al. (17), while studying the radiation decomposition of Deacidite-FF (28) and Zerolitt FF-IP $(16,17)$. Egorov and Novikov (1) also point out that anion exchange resins of the quarternary ammonium type lose their exchange capacity primarily due to the decomposition of functional ion exchange groups. They also suggest that this decomposition is dependent on the nature of the functional groups and their ionic form. Like cation exchangers, the salt forms of anion exchangers are reported to be more radiation resistant compared to hydroxide forms. While there are some reported exceptions to this $(12,16,22)$, generally the nitriate, chloride, chlorate and sulfate forms of anion exchangers are reported to be more radiation resistant $(1,12,16,22,28,29,30)$.

Several of the experimental studies have attempted to examine the loss of exchange capacity of anion exchange resins as a function of absorbed dose. The results of one study by Kazanjian and Horrel (24) are summarized in the illustrations in Figure 8 . It is evident here that both ionic form and the environment of the resin during irradiation influences the radiation chemical changes as measured by the loss of total exchange capacity. The resin irradiated in the nitrate form is much more stable than the chloride forms. The ability of nitrate ions to scavenge hydrated electrons and the strong oxidizing characteristics of chloride radicals are attributed as the reasons for this behavior (24). Anion exchangers with quarternary ammonium base groups, when irradiated, steadily loses the strong base exchange capacity. However, some of the groups 


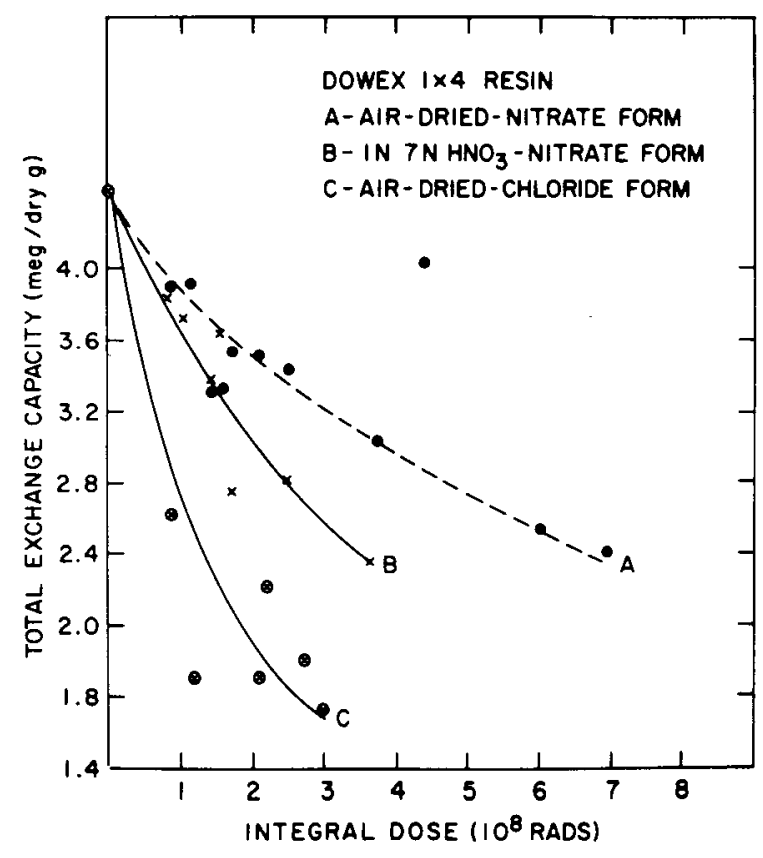

Figure 8. Change in total exchange capacity of a quarternary ammonium type anton exchanger with radiation dose. 
degrade to weakly basic amino groups, which in turn improves the total exchange capacity of the resin $(16,28)$. It is also reported that the irradiation of anion exchangers induces oxidation with the formation of carboxyphenolic and hydroxy groups similar to radiation chemical effects in strongly acidic cation exchangers. This process introduces weakly acidic cation exchange sites in the matrix.

It was pointed out earlier (Section V.1) that several experimental studies on cation exchangers showed that increased cross-linkage of the resin sometimes exhibited greater resistance to radiation damage. This type of relationship between degree of cross-linkage and radiation stability has been apparent in anion exchangers $\mathrm{AV}-17$ and Zerolitt $\mathrm{FF}-\mathrm{IP}(1,16)$. However, an earlier study by Wedemeyer (37) using Dowex-1 resins of various crosslinkages showed maximum degradation (minimum stability) with $4 \%$ cross-linked Dowex-1. Increased radiation stability was apparent in Dowex-l for both higher and lower cross-linkages .

Identification and quantitative analysis of the decomposition products of anion exchange resins during irradiation was undertaken by several investigators $(17,24,28,29,30,31,32,50)$. Quantitative yields of teritiary, secondary and primary aliphatic amines and amonia have been studied. Kiseleva, et al. (50) have reported the results of their analyses of both teritiary and secondary amines from AV-17 anion exchanger under two irradiation conditions. Figure 9 illustrates some of the results on $t$-amine losses from the resin matrix as a function of dose. The amounts of secondary amines identified (not shown in Figure 9) were extremely small compared to the quantities of t-amines. Also included in Figure 9 is the percent weight loss (dotted line) of the resin as a function of absorbed dose. It is obvious that the decrease in weight of these specimens cannot be accounted for by the loss of amino groups alone. Hall and 


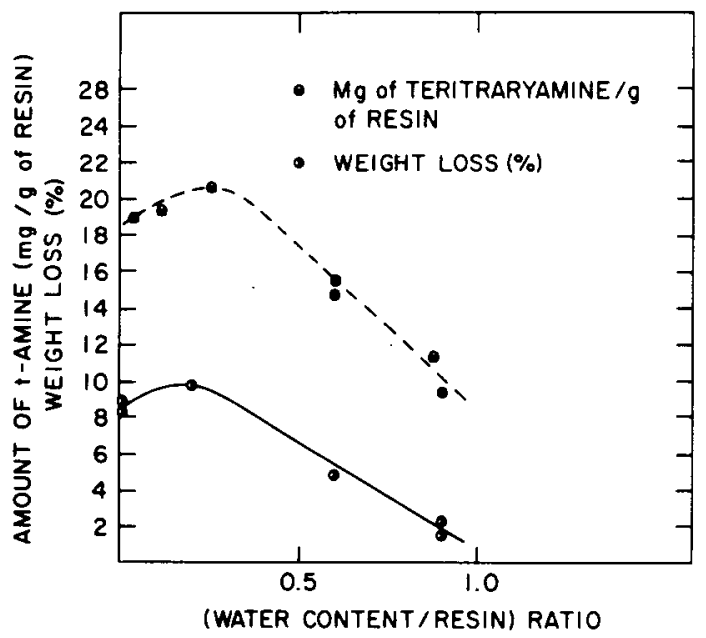

Figure 9. Percent weight loss and the loss of tertiary amine from AV-17 anion exchange resin as a function of absorbed dose. 
Streat (28) in a detailed study of all the basic products (trimethylamine, dimethylamine, methylamine and ammonia) from the anion exchange resin DeaciditeFF reports that all the loss of exchange capacity of this anion exchanger during irradiation can be accounted for by these products alone. Litowska and Narebska (20) using Imac 55-40 anion exchanger have examined the water soluble aliphatic amines and conclude that most of the loss of exchange capacity can be accounted for by deamination rather than other forms of degradation.

It is generally well established that irradiation of anion exchangers with quarternary ammonium groups results in the formation of soluble amines and ammonia, with trimethylamine as the major decomposition product. However, the yield of these products vary in a rather complex manner depending on the moisture content of the resin. Kiseleva, et al. (50) have reported on both the weight loss of the resin and the $10 s \mathrm{~s}$ of amines as a function of water content of AV-17 anion exchange resin. The results of this study, illustrated in Figure 10, was conducted at a radiation dose of $\sim 10^{9}$ rads. The trimethylamine loss from the resin matrix increases steadily up to a point where the water to resin ratio reaches a value of about $0.2: 1$. Beyond this point, further increase in the water content drastically reduces the yield of trimethylamine. The variation in the weight $108 s$ of the resin almost parallels this relationship of trimethylamine production with water content of the resin. These variations cannot be solely explained by the interaction of the resin with the radiolysis products of water, since in this case the dilution law characterizing an indirect action of the radiolysis product of the medium does not hold.

Polycondensation type anion exchange resins are mostly polyfunctional resins of medium and low basicity, with a few exceptions (1). Polycondensation type anion exchangers containing purely aliphatic structural constituents and 


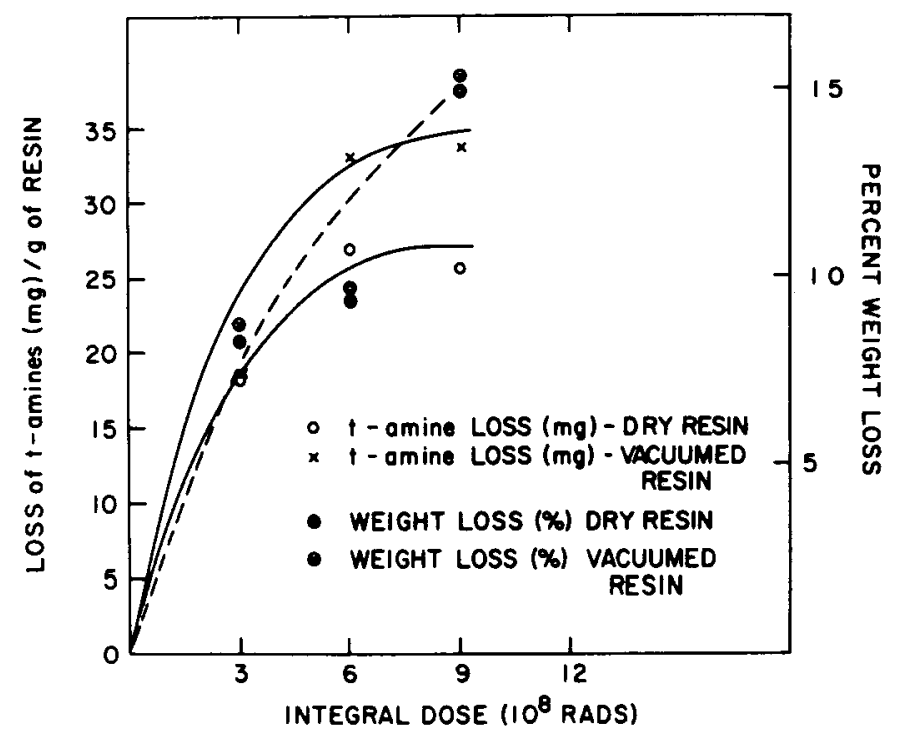

Figure 10. Loss of tertlary amine and percent loss of weight of $\mathrm{AV}-17$ anion exchanger as a function of water content of the resin (at $\left.\sim 10^{9} \mathrm{rads}\right)$. 
those with aromatic groups are common. Those with aromatic groups are less sensitive to radiation damage owing to the protective effect of these groups. Radiation chemistry of polycondensation type resins have been less rigorously explored and many features of their radiolysis remain unexplained.

\section{V.5 High Radiation Resistance of Some Anion Exchange Resins}

A number of studies have demonstrated that anion exchangers containing aromatic systems with conjugated bonds have high radiation stability. Smith and Groh in 1961 reported (22) on the unusually high radiation stability of Permutit SK, a chemically less stable polyfunctional resin derived from copolymerization of styrene, divinylbenzene and pyridine. Since that time several anion exchangers containing pyridine, quinoline and derivatives of pyridine or quinoline have been shown to possess high degrees of resistance to radiation decomposition $(1,30,31,32,53)$.

Resins prepared from 2,5 methylvinyl-pyridine and its substitution products were shown to exhibit no loss of exchange capacity for a total absorbed dose of $\sim 1.4 \times 10^{9}$ rads, when irradiated in water (30). Similar types of radiation resistance were exhibited by resins prepared from quinoline and isoquinoline with chloromethylated styrenedivinylbenzene copolymers under a variety of irradiation conditions. When irradiated in vacuum-dry and air-dry conditions, the exchange capacity of these resins changed by less than $10 \%$ at a total absorbed dose of $\sim 10^{9} \mathrm{rads}$. However, irradiation of these resins in water and $7 \mathrm{~N} \mathrm{HNO}_{3}$ resulted in total exchange capacity 10 sses of $15 \%$ and $55 \%$ respectively at the same dose. Anion exchange resins with pyridine groups show a change in exchange capacity of only about $2.5 \%$ when irradiated in $7 \mathrm{~N}^{\mathrm{HNO}} \mathrm{H}_{3}$ at $\checkmark$ 109 rads. These resins with pyridine exchange groups are more stable to 
ionizing radiations than all the other types of synthetic organic ion exchangers that have been examined for radiation induced chemical changes $(1,30,53)$. V.6. Gas Evolution During the Radiolysis of Ion Exchange Resins

Irradiation of ion exchange resins, just like organic high polymers, result in evolution of gaseous products. This phenomenon is of great importance in the process applications of ion exchange resins, since the gases evolved can seriously interfere with the use of ion exchangers. There are only a few reported experimental studies which have attempted to study the nature of gas evolution during the irradiation of ion exchange resins $(1,23,24,26,27,33$, $42,45,47)$. Of these, the works of Moharcic et. al. $(33,47)$ and Kazanjian et. al. $(23,24)$ have been successful in making some quantitative estimates of the gaseous product formation during the radiolysis of ion exchange resins.

The main gaseous products formed during the irradiation of ion exchange resins are hydrogen and carbon dioxide. Other gases such as carbon monoxide, oxygen, methane, nitrogen, and oxides of nitrogen have been identified. The radiation chemical yields of some of these major products are summarized in Table VII. Most of the results reported are for cation exchangers, but some attempts have been successful in identifying and measuring the gaseous products of radiolysis of anion exchange resins $(24,26)$. During the irradiation of cation ion exchange resins $\mathrm{H}_{2}, \mathrm{CO}_{2}, \mathrm{CO}, \mathrm{SO}_{2}, \mathrm{O}_{2}$ and $\mathrm{CH}_{4}$ have been identified ( 1 , $23,33,47$ ), while $\mathrm{H}_{2}, \mathrm{CO}_{2}, \mathrm{CO}, \mathrm{N}_{2}, \mathrm{~N}_{2} \mathrm{O}$ and No have been reported among the gaseous products of radiolysis of anion exchange resins $(24,26,27)$.

In cation exchange resins, the evolution of hydrogen is a linear function of absorbed dose (47). Hydrogen evolution is also shown to increase with the water content (swelling) of the resin. The results reported by Moharcic and Kramer (47) on Dowex 50W resin are used in the illustration in Figure 11 . The 


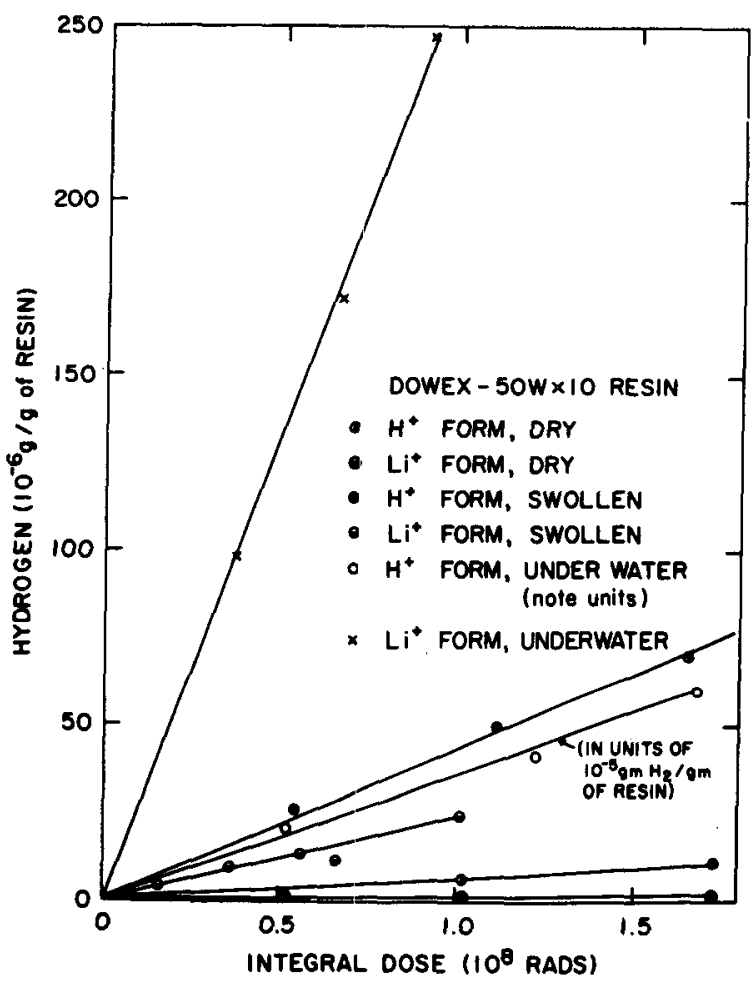

F1gure 11. Evolution of hydrogen during the irradiation of Dowex 50W. 
G-values for hydrogen (see Table VII) evolution from the lithium salt form of this cation exchange resin shows considerable high stability, whereas the $\mathrm{H}^{+}$ form under water shows the least stability toward hydrogen formation.

Sulfur dioxide is formed by the direct action of ionizing radiation on dry forms of sulfonic acid resins. In the presence of water, the $\mathrm{SO}_{2}$ undergoes secondary reactions and for this reason $\mathrm{SO}_{2}$ is not identified during the irradiation of moist and wet resins. The evolution of $\mathrm{SO}_{2}$ during the irradiation of two ionic forms of Dowex 50W in dry and swollen forms reported by Moharcic et. a1. (47) are illustrated in Figure 12. These authors also point out that when a polycondensation type sulfonic resin (Zeo-Karb 215) was irradiated in the dry form, under similar irradiation conditions, only traces of $\mathrm{SO}_{2}$ were identified. The G-values for $\mathrm{SO}_{2}$ formation reported in Table VII are all calculated for the lowest absorbed dose.

Carbon dioxide and carbon monoxide are products of irradiated resins. Like hydrogen, the evolution of $\mathrm{CO}_{2}$ and $\mathrm{CO}$ are quite linear with absorbed dose. Again, the results of Moharcic and Kramer (47) on $\mathrm{CO}_{2}$ and $\mathrm{Co}$ evolution from Dowex 50 are used in the illustrations in Figures 13 and 14 . In general, the radiation chemical yield of carbon monoxide is significantly less than that of carbon dioxide.

Comparative studies done on two commercial cation exchangers, Dowex 50W and Zeo-Karb 215, have demonstrated that polycondensation type cation exchangers are more resistant to gaseous product formation during radiolysis, when compared with styrene-divinylbenzene copolymer resins (47).

Experimental studies identifying and measuring gaseous products of the radiolysis of anion exchangers are very. few, although it is recognized that the irraditons of anion exchangers are accompanied by copious evolution of gases (1, 


\section{Table VII}

G-values for Gaseous Product Formation During the Radiation Decomposition of Ion Exchange Resins

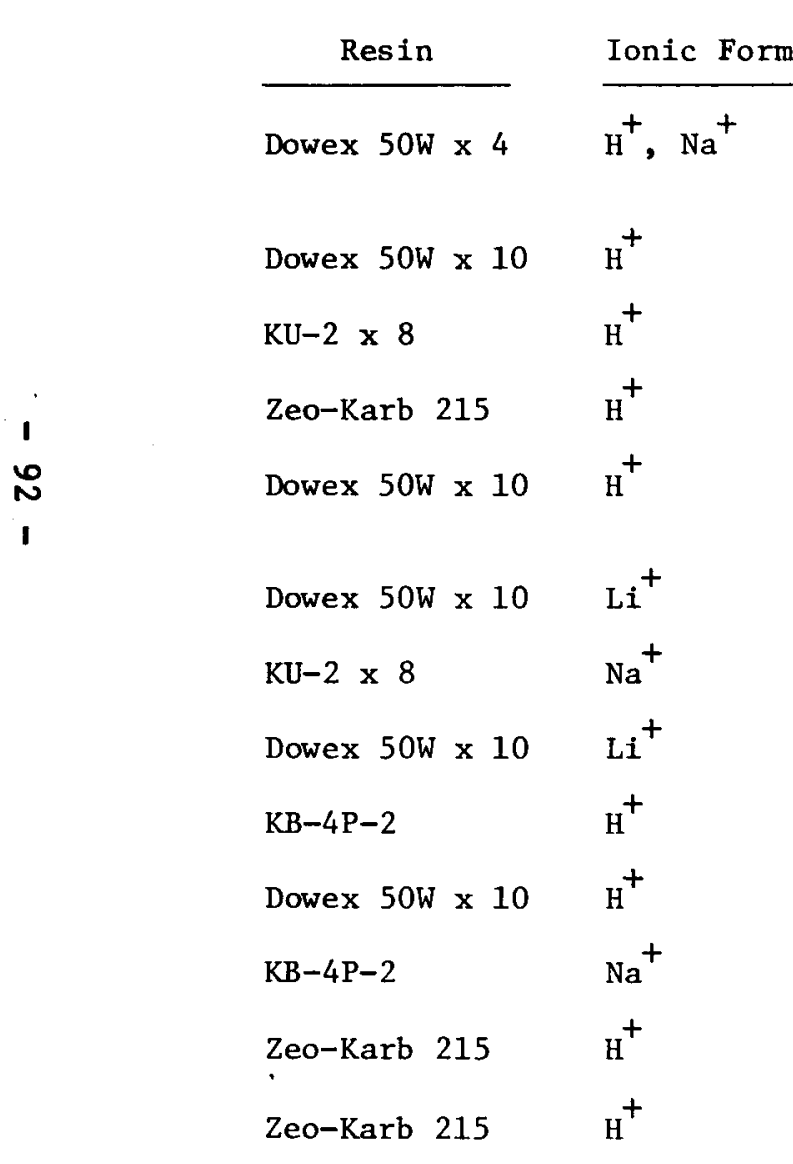

\begin{tabular}{|c|c|c|c|c|c|}
\hline $\begin{array}{l}\text { Irradiation } \\
\text { Condition }\end{array}$ & $\mathrm{G}\left(\mathrm{H}_{2}\right)$ & $\mathrm{G}\left(\mathrm{SO}_{2}\right) *$ & $\mathrm{G}\left(\mathrm{CO}_{2}\right)$ & $\mathrm{G}(\mathrm{CO})$ & Ref. No. \\
\hline $\begin{array}{l}\text { Air-dry } \\
\text { and } 0.1 \mathrm{~N} \text { Hel }\end{array}$ & 0.1 & - & - & - & [23] \\
\hline Dry & 0.026 & 0.087 & 0.035 & 0.009 & [47] \\
\hline Dry & 1.1 & - & 0.06 & - & {$[1]$} \\
\hline Dry & 0.051 & 0.001 & 0.019 & - & [47] \\
\hline $12-47 \%$ water & $\begin{array}{l}0.04 \text { to } \\
0.12\end{array}$ & 20.14 & 20.023 & $\sim 0.022$ & [33] \\
\hline Dry & 0.001 & 0.26 & 0.007 & 0.002 & [47] \\
\hline Dry & 1.0 & - & 0.03 & - & {$[1]$} \\
\hline Swollen & 0.095 & 0.006 & 0.019 & 0.009 & {$[47]$} \\
\hline Swollen & 1.22 & - & 0.60 & 0.39 & {$[1]$} \\
\hline Swollen & 0.095 & 0.006 & 0.019 & 0.027 & [47] \\
\hline Swollen & 3.10 & - & 0.09 & - & {$[1]$} \\
\hline Swollen & 0.12 & 0.005 & 0.046 & 0.005 & [47] \\
\hline $\begin{array}{l}\text { Immersed in } \\
\text { water }\end{array}$ & 1.7 & 0.002 & 0.43 & - & [47] \\
\hline
\end{tabular}


Table VII (Cont'd)

\begin{tabular}{|c|c|c|c|c|c|c|c|c|}
\hline & Resin & Ionic Form & $\begin{array}{l}\text { Irradiation } \\
\text { Condition }\end{array}$ & $\mathrm{G}\left(\mathrm{H}_{2}\right)$ & $\mathrm{G}\left(\mathrm{SO}_{2}\right) *$ & $\mathrm{G}\left(\mathrm{CO}_{2}\right)$ & $G(\mathrm{CO})$ & Ref. No. \\
\hline & Dowex $50 \mathrm{~W} \times 10$ & $\mathrm{H}^{+}$ & $\begin{array}{l}\text { Immersed in } \\
\text { water }\end{array}$ & 1.7 & - & 0.41 & - & [47] \\
\hline & Dowex 50W x 10 & $\mathrm{Li}^{+}$ & $\begin{array}{l}\text { Immersed in } \\
\text { water }\end{array}$ & 1.3 & - & 0.41 & - & [47] \\
\hline & Dowex $1 \times 4$ & $\mathrm{NO}_{3}^{-}$ & Air-dry & 0.09 & - & - & 0.02 & [24] \\
\hline & Dowex 11 & $\mathrm{NO}_{3}^{-}$ & Air-dry & 0.10 & - & 0.06 & 0.01 & [26] \\
\hline & Dowex $1 \times 4$ & $\mathrm{NO}_{3}^{-}$ & In $7 \mathrm{~N} \mathrm{HNO}_{3}$ & 0.02 & - & 0.6 & - & [24] \\
\hline
\end{tabular}

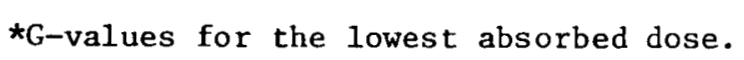




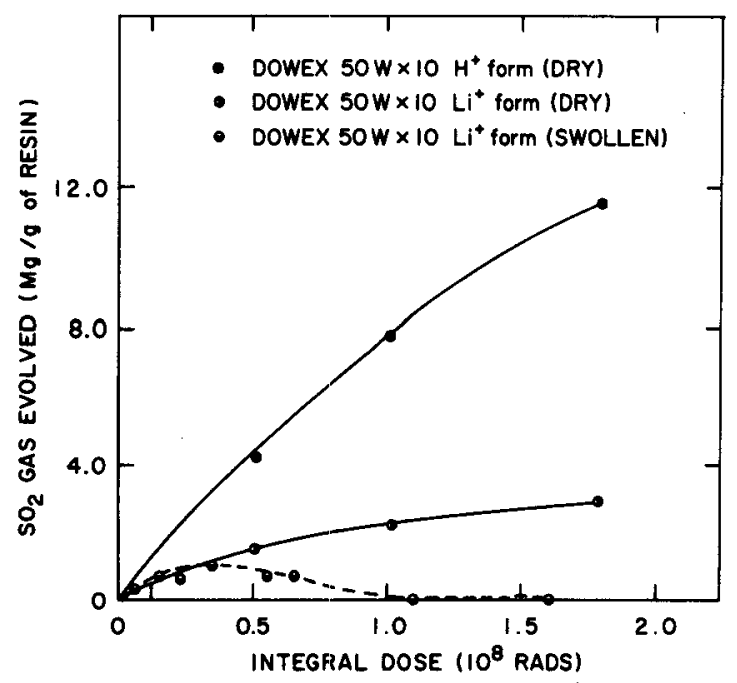

F1gure 12. Sulfur dioxide generation during the radiolysis of Dowex 50W.

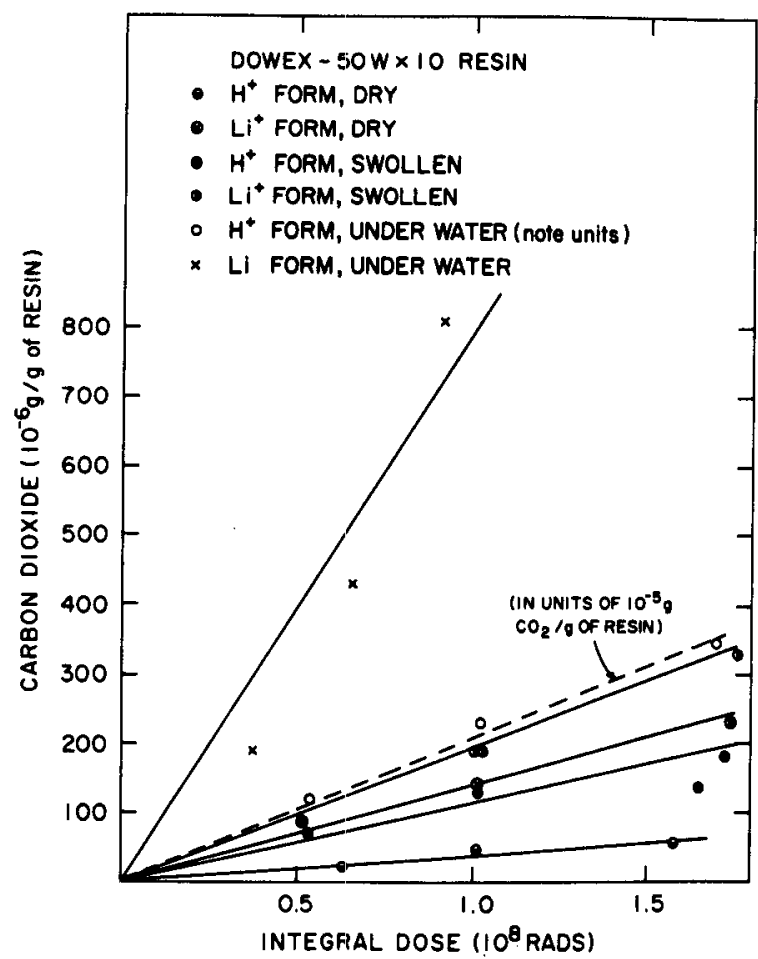

Figure 13. Carbon dioxide evolution during the irradiation of various forms of Dowex $50 \mathrm{~W}$. 


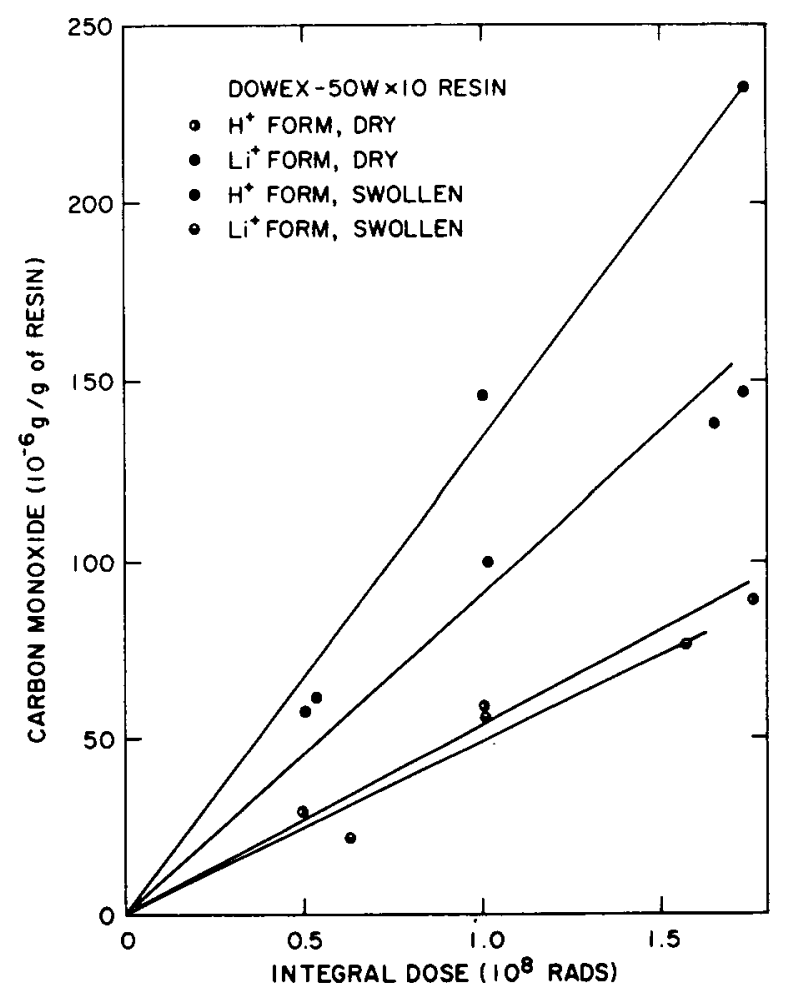

Figure 14. Evolution of carbon monoxide during the Irradiation of two ionic forms of Dowex 50W. 
24, 26). Some of the results of Kazanjian et. al. $(24,26)$ on the evolution of gases during radiolysis of ion exchange resins could be very valuable in predicting potential gas blocks and channeling in ion exchange columns used in intense radiation fields.

V.7 Mechanistic Considerations in the Radiolysis of Ion Exchange Resins

The purpose of this section is to discuss the mechanism of resin radiation damage in the most general terms plausible. The discussion will not cite specific experimental results since the state of knowledge on general mechanisms does not warrent such detail. Rather an overview approach will be taken which will include a general approach for discerning mechanistic details and identify the required specific parameters. The reader is referred to references $(1,21$, $38,46,57,58$ and 66 ) for the experimental details upon which the following discussion is based.

Under the conditions used in waste reprocessing the action of ionizing radiation on resin materials can, at least formally, be classified into two competing processes: direct radiation action on the resin matrix and indirect attack on the exchangers by solvent or solute radiolysis products. Both processes will be a function of reprocessing conditions such as mobile phase composition, contact time, temperature, pressure, packing, density etc. There is a definite absence of general common factors by which one can characterize the susceptibility of a given resin to radiation damage. Radiation studies of both dried resin and heterogeneous mixtures of resins and solutions were considered in formulating this section.

Radiolysis studies of dried resins are useful in terms of discerning direct radiation damage mechanisms. 
Alteration in resin properties such as exchange capacity, acidity, basicity, swelling, solubility, selectivity and exchange kinetics may be a result of functional group cleavage or alteration and/or matrix cross-linking or degradation. The structure and cross-linking properties of resin matrices are important because an increase in the degree of cross-1inking stabilizes functional and matrix groups. This stabilizing behavior is interpreted in terms of the cage effect whereby the radiation produced free radical chain and function group fragments can not escape their cleavage site nor can external species enter this local cage before recombination occurs. Thus the net resin damage per unit radiation dose is less. These cage reactions are represented by reactions (4) and (5) below.

The electronic structure of resin matrix components is important due to energy transfer effects. This behavior manifests itself in the form of different susceptibilities to degradation or cleavage for a given matrix or matrix/function group combination. Resin matrices containing unsaturated linkages, in particular aromatic groups, transfer energy readily to the resin exchange functional groups thereby decreasing resin degradation but increasing functional group loss (i.e., decreasing exchange capacity). Reaction (15) below depicts this energy transfer process. For a given resin susceptible to energy transfer effects, the order of decreasing functional group stability has tended to be: phenolic and sulfonic > secondary and tertiary amine > carboxylic and quaternary ammonium. Due to conflicting results on phosphate and pyridine groups, the relative stability of these groups cannot be presently assigned in the above series. It must be emphasized that general trends such as the above are by no means without exception. For example, the exchange functional group radiation stability of styrene-divinylbenzene resins decreases in the order: 
pyridine > sulfonic and carboxylic > primary, secondary and tertiary amine > quaternary ammonium. Apparently, there is a general tendency for increased susceptibility to cleavage as the degree of substitution on the functional group increases .

The radiolysis of a resin-solution mixture will be a function of the kind of ionizing radiation ( $\alpha, \beta, \gamma$ or $x$-ray), the dose rate and the total dose. The radiation from nuclides during ion exchange stages in a spent fuel reprocessing stream will interact in proportion to the mass energy absorption coefficient ratios (for $\gamma$ - and $x$-rays) or mean mass stopping power ratios (for $\alpha$ or beta particles) of the compounds, molecules or functional groups comprising the stream. For materials of similar composition these absorbed dose ratios can be approximated by the electron density ratios for the stream components. The production reactions of reactive species by radiation for an ion exchange column are:

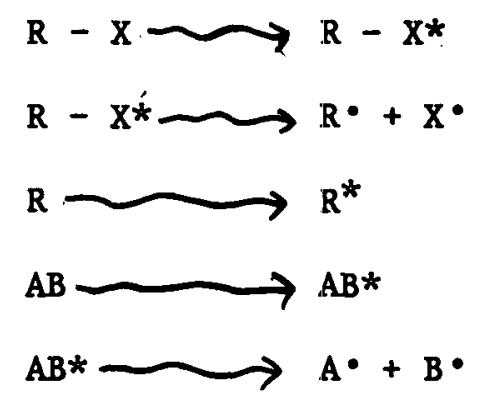

where $R$ - X represents any ion exchanger with functional group $X, R$ denotes any resin matrix component, $A B$ represents any solvent or solute component, the dot by a symbol denotes a free radical species, the asterisk denotes an excited species and the wavy reaction arrows conotate interaction between a species and one of the forms of radiation. 
The initial populations of the radiation generated reactive species (i.e., the products in the above reactions) will be a function of the absorbed dose ratio for a given species, the absorbed dose rate $I(t)$ and the radiation chemical yield $G(t)$ where $t$ denotes a possible time dependent function. The fraction of the initially generated species population available for net reaction with the ion exchange resin will depend upon the reaction rates for loss of reactive species

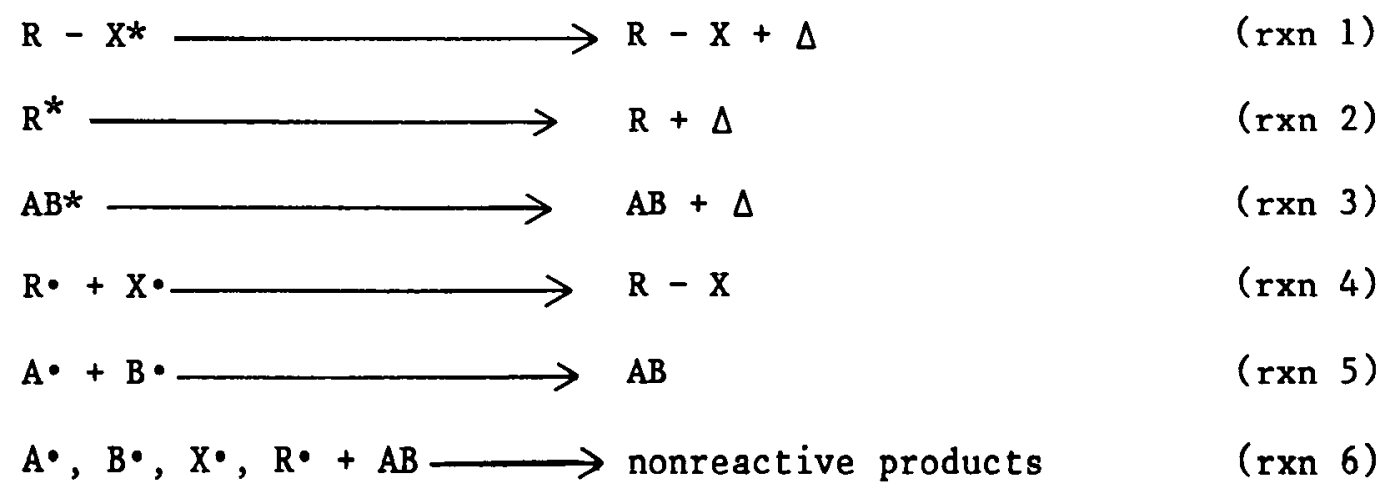

and upon reactions of the initial species with solvent, solute or resin to form new reactive species $\mathrm{P}\left(\mathrm{e} . \mathrm{g} ., \mathrm{H}+\mathrm{H}_{2} \mathrm{O} \longrightarrow \mathrm{H}_{2}+\mathrm{OH}\right)$. The delta sign $\Delta$ in the above equations denotes energy release in the form of heat or 1ight. Note that each of the above reactions represents one reaction class such that there could, and probably will be, several reactions in each class.

Not all of the above reactions will be competitive. Some rates will be much faster than the average such that they will constitute the rate determining steps in producing reactive species for resin attack. For example, the direct interaction of the ionizing radiation can be eliminated for minor stream components based on electron densities. If the reaction rate constants for the loss of species reactions are known the mechanism can be further simplified. It is 
at this point that the lack of basic knowledge prevents clarification of the resin radiation damage mechanism. Experimental work is needed to identify the yields and kinetics of these reactive species.

The reactions that the reactive species can undergo with the resin are:

$$
\begin{aligned}
& \mathrm{AB}+\mathrm{R}-\mathrm{X}^{*} \longrightarrow \mathrm{R}-\mathrm{A}+\text { products } \quad \text { (rxn 10) } \\
& \mathrm{AB}+\mathrm{R} * \longrightarrow \mathrm{R}-\mathrm{B}+\text { products } \quad \text { (rxn 11) } \\
& \mathrm{R}-\mathrm{X}+\mathrm{R}-\mathrm{X}^{*} \longrightarrow \mathrm{R}-\mathrm{R}+\text { products } \quad \text { (rxn 12) } \\
& \mathrm{R}+\mathrm{R}-\mathrm{X}^{*} \longrightarrow \mathrm{R}-\mathrm{R}+\text { products } \quad \text { (rxn 13) } \\
& R+R^{*} \longrightarrow R-R+\text { products } \quad \text { (rxn 14) } \\
& \mathrm{R}-\mathrm{X}^{*}+\mathrm{R} \longrightarrow \mathrm{R}-\mathrm{X}+\mathrm{R}^{*} \quad \text { ( } \mathrm{rxn} 15 \text { ) } \\
& R^{\bullet}, X^{\bullet}, A^{\bullet}, B^{\bullet}, P+R-X \longrightarrow \text { products (rxn 16) } \\
& R^{\bullet}, X^{\bullet}, A^{\bullet}, B^{\bullet}, P+R \longrightarrow \text { products (rxn 17) }
\end{aligned}
$$

Here again the mechanism cannot presently be simplified since the relevant rate constants are not known. Note that specific study of each resin under varying reaction conditions would not be required. If the various classes of the above reactions were sufficiently characterized such that the rate determining steps could be identified and representative rate parameters for these steps were known, a kinetic model could be built for predicting the radiation damage behavior of resins under varied process conditions.

The utility and attainability of using individual chemical reactions to build a mechanistic model which describes and predicts the course of complex chemical systems is exemplified by results obtained by atmospheric chemical 
studies. The number of possible chemical reactions which can occur in the atmosphere is large in comparison to those for a nuclear waste reprocessing stream. Yet through careful experimentation the more important atmospheric reactions have been identified and formulated into mechanistic models. These studies reveal that using the individually determined rate parameters one can predict data that agrees with the results observed in the atmosphere. Thus the very complex and uncontrolled (by man) chemistry of the atmosphere can be described and predicted from an understanding of the basic chemistry. For nuclear reporcessing steams, where the reaction conditions are carefully controlled, similar predictive modeling can be realized. Before such useful modeling can be achieved, more experimental work on the basic reactions important to reprocessing must be done.

In the absence of such information one has to look for emperical relationships based on known resin studies. This approach is at best tenuis since one can not be sure that emperical relationships based on one set of data for a resin also holds for this same resin under different conditions (much less for a new resin). This, to a large part, is exemplified by the extensive inconsistancy of the literature data. Of the numerous equations cited in the literature most can be formulated into one of two types of relationships. Both relationships attempt to describe a given resin properties, for example, the behavior of resin capacity $\mathrm{C}$, as a function of total radiation dose $\mathrm{D}$. One is based on an exponential relation between $C$ and $D$ :

$$
c=c_{0} e^{-k D}
$$

where $C_{0}$ is the initial resin capacity and $k$ is a coefficient for a given resin 
under particular radiation conditions with units of recipricol dose. For low dose conditions $k$ is related to the initial radiation chemical yield for the loss of resin capacity $G_{0}$ :

$$
k=\frac{G_{0} \times 10^{-9}}{C_{0}}
$$

where the factor of $10^{-9}$ is required for unit conversions.

The second type of emperical relationship describes the decrease in capacity as a linear function of dose:

$$
C=C_{0}-B D
$$

where $B$ is a coefficient which depends on the nature of the resin and the condition of irradiation with units of capacity per dose. For low dose conditions $\beta$ is related to $G_{0}$ :

$$
\beta=G_{0} \times 10^{-9}
$$

and is thus equivalent to $\mathrm{k}_{0}$. In fact, the linear equation 2 is just the first two terms in the series expansion of the exponential equation 1 , and as such is just an approximation of the latter.

The values of $G_{0}$ given in Tables III and IV have been classified with respect to the nature of the resin damage:

i) for $G_{0}=0.1-0.01$ the radiation energy absorbed by the matrix and functional groups is dissipated as heat resulting in little damage to the resin. 
ii) for $G_{0}=1-5$ the radiation energy absorbed directly by the functional groups results in their decomposition

iii) for $G_{0}=5-15$ the radiation energy absorbed by the matrix is transferred to the functional groups which thus undergo indirect radiation decomposition in addition to the direct radiation decomposition above. Data which follows the exponential type relationship has been sited as evidence that the main radiation process is destruction of functional groups due to their direct absorption of the radiation energy. The linear relationship has been interpreted as evidence that the main radiation process leading to functional group loss is energy transfer from the matrix to the functional groups. Such classifications of $G_{0}$ and general mechanistic statements ignore indirect processes due to radiation produced solvent or solute species and should be viewed in that light. Furthermore, the above relationships in general only hold for low degrees of radiation damage.

Resin degradation processes may take place even after irradiation is terminated due to long lived reactive species formed by various chemical reactions. For example, radiation induced oxidation can give peroxides which will subsequently decompose and react with a resin. Any mechanism of practical use must take post irradiation effects into account. This can be done if the basic types of reactions that occur are identified and kinetically characterized. Once identified it would not be unexpected that the kinetics of a large fraction of the post reactions could be extracted from existing literature.

It must be stresed that the emperical relationships and equations are hypothetical. Low accurancy of parameters and poor reproducibility of experimental results are a typical feature of the radiation chemistry of ion exchange 
resins. This is a result of both the complexity of the systems and of the lack of standardized preparation, conditioning, testing and irradiation procedures. Reliable mechanistic characterization of the radiolysis of ion exchange resins must wait more uniform and more basic experimentation. 


\section{CONCLUSIONS}

For more than three decades there have been extensive applications of ion exchange materials in the nuclear process industry. Process related problems of serious nature caused by radiation chemical changes in the ion exchange medium have been occasionally mentioned in the open literature as well as in safety evaluation studies of radioactive material processing $(106,108)$. The present literature survey identified a number of reports on experimental studies to evaluate the process related properties of organic ion exchangers. Still, there are numerous problems associated with the radiation chemical changes of ion exchange materials that are not properly understood. Thus, in spite of the apparent abundance of experimental studies, there is no reliable information to allow one to predict the radiation chemical effects on process applications of ion exchange materials in the nuclear industry.

The radiation stabilities of a variety of ion exchange resins have been studied by exposing dry, moist or wet resins to a variety of ionizing radiation sources, both internal and external to the resin. The early works of Wedemeyer (37), Higgins (94), Fisher (34), Payne (48) and Utley (50) during the 1950's have been repeatedly mentioned in numerous publications on the effects of intense radiation on ion exchange materials. In spite of the recognized limitations of these early investigations using a few of the important ion exchangers of that period, these studies remain primarily the basis for radiation damage evalution of ion exchange materials used in the U.S.

The need to fully understand the effects of radiation in process applications of ion exchange materials is apparent from the increasing number of reports in the open literature on process related incidents ( 99 to 108). From 
the present survey of current knowledge on the radiation effects on ion exchange materials, it can be concluded that:

(i) The fundamental processes involved in radiation damages to ion exchange materials are not understood

(ii) structural and chemical constituent modifications can significantly change the radiation resistance of ion exchange materials

(iii) there is a serious gap in systematic investigations to develop highly' radiation resistant inorganic ion exchangers of high exchange capacity

(iv) there is a need to extend the radiation effect studies to include investigations of secondary reactions of the radiolysis products of all the materials in a process system

(v) radiation-resistant and process-efficient organic and inorganic ion exchangers tailored to the special needs of radiochemical processing in the nuclear industry need to be developed.

Recent work on radiation chemical effects on ion exchangers from the U.S. $(14,23,24,25)$ are few and these are simply attempts to examine a process related problem of immediate concern to these investigators. Almost all the recent work (see Reference list, Section VII of this report) published in the open literature on radiation stability of ion exchangers are from the Soviet Union and East European countries and involve the examination of ion exchangers that are of interest to their process industry. Because of the proprietary nature of comercial resin manufacture and the nature of the trade nomenclature of ion exchange materials, it is difficult to examine the possible applications of Soviet experimental studies to ion exchange materials that are used in the U.S. However, with an accurate knowledge of the generic chemical nature of the ion 
exchange resin to be used in a process application, the data assembled here can be profitably used to gain knowledge about the effects of radiation on process materials. 
1. E. V. Egorov and P. D. Novikov, "Action of Ionizing Radiation on Ionexchange Materials," Atomizdat, Moscow (1965), Israel Program for Scientific Translations, Jerusalem (1967).

2. I.A.E.A. Technica1 Report Series No. 78, "Operation and Control of Ionexchange Processes for Treatment of Radioactive Wastes," International Atomic Energy Agency, Vienna (1967).

3. P. G. Clay, G. R. Hall, M. K. Rahman and M. Rashid, "The $\gamma$-Radiolysis of Zeocarb-225 Cation-exchange Resins," J. App1. Polymer Sc1. 14, 2167-70 (1970).

4. W. C. Yee and W. Davis, Jr., "Effects of Gamma Radiation on Cation Exchange Resin in a Flowing-Water System," Nuclear Sclence and Eng. 24, 1-5 (1966).

5. Idem, ORNL-P-661 (1964).

6. M. A. Kskarov, A. T. Dzhalilov, R. A. Nazinova and G. S. Tsveshko, "Radiation Stability of Polycondensation Ion-exchange Resins Based on Furfural," Translation from Zhurnal Priklodnoi Khimi, 46(9), 2115-17 (1973).

7. E. A. Chuvela, P. P. Nazarov and K. V. Chmutov, "The Use of Radiation Resistant Resins for Separation of Radioactive Elements by Displacement Chromatography-I. Separation in KU-4 Cation Exchange Resin with the Aid of Diethylenetriamine Pentaacetic Acid," Russ. J. Phys. Chem. [English Translation] 50(5), 794-796 (1976).

8. Idem, -II, Separation on $\mathrm{KU}-6$ and $\mathrm{KU}-160$ Cation Exchange Resins with the Aid of Diethylenetriamine Pentaacetic Ac1d," Ibid., 50(5), 796-798 (1976).

9. K. M. Semushin, I. P. Galitskaya, and I. A. Kuzin, "Radiation and Chemical Stability of Methacrylic Acld-Divinylbenzene Copolymers," Translation from Zhurnal Priklodnol Kh1mi, 44(9), 2035-39 (1971).

10. T. Ichikawa and Z. Hagiwara, "Effect of Gamma Radiation on Cation Exchange Resin," J. Nuc1. Sci. Tech. 10, 746-52 (1973).

11. V. Dornow, S. Specht, W. Welnlander and H. J. Born, "Modification of the Efficlency of Chromatographic Systems During the Transition to Higher Activities - I. Comparison of $\alpha$ - and $\gamma$-Radiolysis of a Cation Exchanger wh th Respect to Modifications in its Weight, its Salt-Splitting Capacity, its Residual Capacity and 1ts Expansion Capacity," J. Radianal. Chem. 24, 393-409 (1975).

12. T. Shigematsu and T. Oshio, "Effect of ${ }^{60}$ Co Gamma Radiation on Ion Exchange Resin," Bul1. Inst. Chem. Research, Kyoto University (Japan), 37, 349-52 (1959). 
13. E. W. Bauman, "Radiolysis of Ion Exchange Resins Used in the Purification Systems of Savannah River Reactors," U.S. AEC Report: DP-977, Savannah River Laboratory, Aiken, S. C. (July 1965).

14. E. W. Bauman, "Gamma Irradiation of Individual and Mixed Ion-Exchanger Resins," J. Chem. Eng. Data 11, 256-260 (1966).

15. E. W. Bauman, "Depletion of Deionizer Resin in an SRP Reactor System," U.S. AEC Report: DP-973, Savannah River Laboratory, Aiken, S. C. (1965).

16. A. Basinski, A. Narebska and M. Tempczyk, "Effect of Gamma Radiation on Ion Exchange Resins. I-Radiation Damage of Functional Groups in the Strongly Basic Anion-Exchange Resin Zerolit FF-IP," Nukleonika (English Translation) 14(5), 77-88 (1969).

17. A. Narebska, A. Basimski and M. Litowska, "Effect of Gamma Radiation on Ion Exchange Resins. II-Investigation of the Effect of Gamma Radiation on Zerolit FF-IP by the IR-Method," Nukleonika (English Translation) 15(2), 30-39 (1970).

18. A. Narebska and B. Mazurkfewicz, "Effect of Gamma Radiation on Ion Exchange Resins. IV-Degradation and Cross-Linking of Polystyrene Sulfonic Cation Exchange Resin, Zerolit-225 of Different DVB Contents," Nukleonika (in Polish) 27(3-4), 169-179 (1972).

19. M. Litowska and A. Basimski, "Effects of Gamma Radiation on Ion Exchange Resins. V-Kinetics of Radiation Damage of Strongly Alkaline Functional Groups in Anion Exchange Resin Zerolit FF-IP," Nukleontka (English Translation) $18(5), 1-6$ (1973).

20. M. Litowska, A. Narebska and J. Ostowska, "Effects of Gamma Radiation on Ion Exchange Resins - VI. The Kinetics and Mechanism of Radiation-induced Décomposition of Functional Groups in an IMAC S5-40 Anion Exchange Resin," Nukleonika (English Translation) 19(1), 19-29 (1974).

21. M. Litowska and J. Ostrowska, "The Effects of Radiation on Ion Exchangers VII. The Kinetics of Variation in Sulfonic Groups of the Cation Exchanger IMAC-C12," Nukleonika (in Polish) 19(4), 284-290 (1974).

22. L. L. Smith and H. J. Groh, "The Effect of Gamma Radiation Ion Exchange Resins," USAEC Report DP-549, Savannah River Laboratory, Aiken, S. C. (1961).

23. A. R. Kazanjian and D. R. Horrell, "Radiation Effects on Ion-Exchange Resins - I. Gamma Irradiation of Dowex 50 XW," USAEC Report RFP-2140, Rocky Flats Division, Golden, Colorado (1974).

24. A. R. Kazanjlan and D. R. Horre11, "Radiation Effects on Ion-Exchange Resins - II. Gamma Irradiation of Dowex 1," USAEC Report RFP-2345, Rocky Flats Division, Golden, Colorado (1975). 
25. A. R. Kazanjlan and D. R. Horreli, "Radiation Effects on Ion-Exchange Resins - III. Alpha Irradiation of Dowex 50W," U.S. AEC Report RFP-2298, Rocky Flats Division, Golden, Colorado (1975).

26. A. R. Kazanjian, Private Communication.

27. A. R. Kazanjian, Private Communication.

28. G. R. Ha11 and M. Streat, "Radiation-induced Decomposition of Ion-Exchange Resins, Part I. Anion-Exchange Resins," J. Chem. Soc. B, 5205-5211 (1963).

29. M. T. Ahmed, P. G. Clay and G. R. Hall, "Radiation-induced Decomposition of Ion-Exchange Resins, Part II. The Mechanism of the Deamination of Anion-Exchange Resins," J. Chem. Soc. B, 1155-1157 (1966).

30. E. D. Klseleva, K. V. Chmutov, V. M. Khasanova and Z. A. Markova, "Radiation Stability of AN-25 and AN-25A Anion-Exchange Resins," Russian J. of Phys. Chem. (English Translation) 45(4) 499-502 (1971).

31. E. D. Kiseleva, V. M. Khasanova and K. V. Chmutov, "The Radiation Stability of AVKH-8P and AVI-8P Quinoline and Isoquinoline Anion-Exchange Resins," Russian J. Phys. Chem. (English Translation) 45(5) 667-669 (1971).

32. V. M. Khasanova, E. D. Kiseleva and K. V. Chmutov, "An Infrared Spectroscepic Study of the Change in the Structure of AVKh-8P and AVI-8P AnionExchange Resins Under the Influence of Ionizing Radiation," Russian J. Phys. Chem. (English Translation) 45(5) 725-726 (1971).

33. G. Mohorcic, V. Kramer and M. Pregelj, "Interaction of a Sulfonic Acid Ion Exchange Resln with Tritiated Water on $\gamma$-Irradiation," Int. J. App1. Rad. and Isotopes, 25, 177-182 (1974).

34. S. A. Fisher, "Effect of Gamna Radiation on Ion Exchange Resins," USAEC Report RMO-2528 (1954).

35. G. J. Moody and J. D. R. Thomas, "The Stability of Ion Exchange Resins: Part II - Radiation Stability," Laboratory Practice, 21(10), 717-722 (1972).

36. J. A. Masinsky and A. J. Giuffrida, "The Radiation Stability of IonExchange Materials," USAEC Report: ORNL-1978, Oak Ridge National Laboratory (1957).

37. R. E. Wedemeyer, "The Stability of Ion Exchange Resins to X-Rays," Ph.D. thesis submitted to faculty of graduate school of Vanderbilt University, Nashville, Tennessee (Sept. 1953). 
38. E. D. Kiseleva, K. V. Chmutov and N. V. Kuligina, "The Mechanism of the Effect of Radiation on KU-2 Cation-Exchange Resin," Russian Journal of Physical Chemistry, [English Translation], 44(2), 264-267 (1970).

39. E. D. Kiseleva, K. V. Chmutov and M. M. Klientovskaya, "Action of Ionizing Emissions from a Stream of Accelerated Electrons on Polycondensation CationExchange Resins," Russ. J. Phys. Chem. [English Translation] 42(11), 151820 (1968).

40. P. E. Tulupov and A. I. Kasperovich, "Effect of the Structure of Macromolecules of Sulfonic Actd Cation-Fxchange Resins in Their Stability to Radiation," Russian J. Phys. Chem. [English Translation] 42(8), 1040-1043 (1968).

41. E. D. Kiseleva, K. V. Chmutov, M. M. Klientovskaya and V. P. Li, "Radiation Stability of KU-4 Cation-Exchange Resin," Russian J. Phys. Chem. [English Translation] 41(7), 850-853 (1967).

42. 0. R. Skorokhod and I. B. Stankevich, "Radiation Stability of the Sulfonated Cation-Exchange Resin KU-2 in the Lithium, Sodium and Potassium Forms," Russian J. Phys. Chem. [English Translation] 42(2), 271-273 (1968).

43. P. E. Tulupov, A. M. Butaev, V. P. Yreben and A. I. Kasprovich, "Effect of Content of Divinylbenzene in KU-2 Cation-Exchange Resin on its Resistance to Irradiation in Water," Russian J. Phys. Chem. [Eng1ish Translation] $47(4), 551-553$ (1973).

44. A. V. Nikolaev, I. N. Gribanova, N. I. Yakovleva and I. D. Khol'kina, "Radiation Stability of Complex Forming Organophosphorus Resins," Russian J. Phys. Chem. [English Translation] 40(4), 456-57 (1966).

45. I. N. Gribanova, I. D. Khol'kina, Yu. N. Polovinkin and A. V. Nikolaev, "Radiation, Chemical and Mechanical Stability of Porous Organophosphorus Cation Exchangers," Russian J. Phys. Chem. 44(7), 983-985 (1970).

46. R. H. Wiley and G. Devenuto, "Irradiation Stability of Sulfonated Styrene Resins Cross-1inked with Various Divinylbenzene Isomers and Mixtures Thereof," J. Appl. Polym. Sc1. 9, 2001-2007 (1965).

47. G. Mohreic and V. Kramer, "Gases Evolved by ${ }^{60}$ Co Radiation Degradation of Strongly Acidic Ion Exchange Resins," J. Polymer Science, Part C, No. 16, 4185-4195 (1968).

48. J. Payne, "Investigation of the Mechanism of the X-Ray Degradation of Dowex-50 Ion-Exchange Resin," M.A. thesis submitted to the faculty of the graduate school of Vanderbilt University, Nashville, Tennessee (March 1956).

49. J. W. Utley, "The Chemical Effects of Low Energy X-Radiation on IonExchange Resins," Part I of Ph.D. thesis submitted to the faculty of the graduate school of Vanderbilt University, Nashville, Tennessee (June 1959). 
50. E. D. Kiseleva, K. V. Chmutov and N. V. Kuligina, "The Mechanism of the Effect of Ionizing Radiation on AV-17 Anion-Exchange Resin," Russian Journal of Physical Chemistry [English Translation] 44(2), 261-263 (1970).

51. E. D. Kiseleva, K. V. Chmutov and V. N. Krupnova, "Effect of Accelerated Electrons on KU-2 Cation-Exchange Resin - I," Russian J. Phys. Chem. [English Translation] 35(8), 892-895 (1961).

52. Ibid. 35(8), 895-898 (1961).

53. E. D. Kiseleva, K. V. Chumutov and N. V. Filatora, "Radiation Stability of Ion-Exchange Resins - III, Action of Ionizing Flux on Accelerated Electrons of Anion-Exchange Resins," Russian J. Phys. Chem. [English Translation] $36(11), 1335-37$ (1962).

54. E. D. Kiseleva, K. V. Chumutov and M. M. Klientovskaya, "The Radiation Stability of KU-5G, KU-6G and EO-7 Polycondensed Cation Exchange Resins," Russian J. Phys. Chem. [English Translation] 39(3), 406-407 (1965).

55. E. D. Kiseleva, A. V. Ragimov, K. V. Chmutov, A. A. Berlin, M. M. Klientovskaya and T. A. Bryushkova, "Action of Accelerated Electrons on Polysulfophenylenequinone Cation-Exchange Resins," Russian J. Phys. Chem. [English Translation] 38(9), 1255-1257 (1964).

56. S. V. Tabakova, E. D. Kiseleva and K. V. Chmutov, "Radiation Stability of VP-K Ion Exchanger," Russian J. Phys. Chem. [English Translation] 49(2), 279-281 (1975).

57. E. D. Kiseleva and K. V. Chmutov, "The Mechanism of the Radiation-induced Oxidation of AV-18 Anion-Exchange Resin," Russian J. Phys. Chem. [English Translation] 44(10), 1443-1446 (1970).

58. E. D. Kiseleva, K. V. Chmutov and V. N. Krupnova, "Radiation Stability of Ion-Exchange Resins," Russian J. Phys. Chem. [English Translation] 36(11), 1331-1335 (1962).

59. I. A. Kuzin, A. M. Semushin, V. V. Antuf'ev, M. P. Votinov and V. F. Evdokimov, "Processes Occurring in KB-4P-2 Cation Exchanger Under the Influence of $\gamma$-Radiation from Cobalt-60," Russ. J. Phys. Chem. [English Translation] 36(2), 205-207 (1962).

60. N. V. Kuligina, E. D. Kiseleva and K. V. Chmutov, "Strontlum-Hydrogen Exchange Statistics on Irradiated KU-2 Cation Exchange Resin - I, StrontiumHydrogen on KU-2 Irradiated in Air-Dry State and in Vacuum," Russ. J. Phys. Chem. [English Translation] 50(1), 85-87 (1976).

61. Idem, -II, Strontium-Hydrogen Exchange Statistics on KU-2 Irradiated with Different Water Contents," Ibid. 50(1), 87-88 (1976).

62. E. D. Kiseleva, S. V. Tabakova and K. V. Chmutov, "Kinetics of the Sorption of the Hexanitratoplutonate Anion on Irradiated AN-24 Anion-Exchange Resin," Russian J. Phys. Chem. [English Translation] 50(9), 1419-1421 (1976). 
63. Idem, 1bid., 50(, $1421-1423$ (1976).

64. E. D. Kiseleva, V. M. Khasanova and K. V. Chmutov, "An Infrared Spectroscopic. Study of the Changes in the Structure of VP-1A Anion Exchange Resin. on Irradiation," Russ. J. Phys. Chem. [English Translation] 45(5), 669-674 (1971).

65. E. D. Kiseleva and K. M. Chmutov, "An Infrared Spectroscoplc Study of the Changes in the Physicochemical Structure of Irradiated AV-17 Anton Exchange Resin," Russ. J. Phys. Chem. [English Translation] 45(6), 850-853 (1971).

66. A. M. Semushin, I. A. Kuzin, V. V. Antuf'ev, M. P. Votinov and V. F. Evidokimov, "Electron Paramagnetic Resonance Study of the $\gamma$-Irradiated Cation Exchangers KU-2, SF, KU-1 and SBS," Russ. J. Phys. Chem. [English Translation] 36(7), 837-838 (1962).

67. T. A. Karpukhina, E. D. KIseleva, K. V. Chmutov and M. P. Glazunov, "Electron Spin Resonance Investigation of the Action of Cobalt-60 $\gamma$-Rays on Anion Exchange Resins," Russ. J. Phys. Chem. 40(11), 1529-1532 (1966).

68. T. A. Karpukhina, E. D. Kiseleva and K. V. Chmutov, "Electron SpIn Resonance Study of the Low-Temperature Radiolysis of Ion Exchange Resins - I, the Study of the Relative Radiation Stabilities of Sulfonic Acld Cation Exchange Resins," Russ. J. Phys. Chem. [English Translation] 50(4), 558-561 (1976).

69. Idem, -II, KU-2 Cation Exchange Resin, ibid., 50(5), 712-714 (1976).

70. G. M. Armitage and S. J. Lyle, "Mass Spectrometric Study of the Deterioration of Polystyrene-based Ion Exchangers," Talanta, 20, 315-320 (1973).

71. P. E. Tulupov, "Kinetics of the Scission of the Functional Groups of Ion Exchange Resins - VIII. Thermal and Radiation Stability of Ankalit KT-3 Sulfonic Acid Cation Exchange Resin in Water," Russ, J. Phys. Chem. [English Translation] 47(2), 194-197 (1973).

72. P. E. Tulupov, A. M. Butaev, V. P. Yreben and A. I. Kasperovich, "Kinetics of Desulfuration of the $\mathrm{H}$-form of $\mathrm{KU}-2$ Cation Exchange Resins in Water by Simultaneous Heat Treatment and Irradiation," Russ, J. Phys. Chem. [English Translation] $46(1), 74-77$ (1972).

73. P. E. Tulupov, "Thermal Stability of Sulfonated Cation Exchange Resins in a Medium of Tertlary Pentenes," Russ. J. Phys. Chem. [Engl1sh Translation] $39(11), 1447-1450$ (1965).

74. P. E. Tulupov, E. I. Lyustgarten and I. G. Stebeneva, "Thermal and Radiation Stability of Macroporous Sulfonic Actd Cation Exchange Resins Based on Styrene-Divinylbenzene Copolymers," Russ. J. Phys. Chem. [English Translation] 46(5), 689-693 (1972). 
75. B. S. Roginskaya and N. V. Bychkov, "Effect of Water of Swelling on the Thermal Stability of AV-17 Anion Exchange Resin in the Chloride Form," Russ. J. Phys. Chem. [English Translation] 50(9), 1427-1428 (1976).

76. V. K. Shiyaev and A. I. Kasperovich, "Effect of Ion Exchange Kinetics on Thermolysis (Radiolysis) of Mixed Forms of Exchangers," Russ. J. Phys. Chem. [English Translation] 49(4), 546-547 (1975).

77. K. H. Lin, "Use of Ion Exchange for the Treatment of Liquids in Nuclear Power Plants," U.S. AEC Report: ORNL-4792 (Dec. 1973).

78. G. E. Boyd and J. Schubert, "The First Use of Organic and Inorganic Ion Exchangers for Separating Plutonium from Uranium and the Fission Products," in Vol. 4 of Progress in Nuclear Energy Series III, Process Chemistry, eds. C. E. Stevenson, E. A. Mason and A. T. Gresky, Pergamon Press, New York, N. Y., pp. 319-343 (1970).

79. V. Veselý and V. Pekárek, "Synthetic Inorganic Ion-Exchangers - I," Talanta, 19, 219-262 (1972).

80. V. Pekárek and V. Vesely, "Synthetic Inorganic Ion-Exchangers - II," Talanta, 19, 1245-1283 (1972).

81. C. B. Amphlett, "Inorganic Ion Exchangers," Elsevier Publishing Co., New York, N. Y. (1964).

82. N. Michael, W. D. Fletcher, M. J. Bell and D. E. Croucher, "Inorganic Ion Exchange Materials for Water Purification in CVTR," Carolinas Virginia Nuclear Power Associates, Inc., Charlotte, N. C., Report 非CVNA-135, Westinghouse Electric Company, Atomic Power Department, Pittsburgh, Pa. (Aug. 1961).

83. B. P. Robinson, "Ion-Exchange Minerals and Disposal of Radioactive Wastes A Survey of Literature," Geological Survey Water-Supp1y Paper \#1616, U. S. Department of the Interior, Superintendent of Documents, U. S. Govt. Printing Office, Washington, D. C. (1962).

84. R. L. Hemmings, "New Materials for Ion-Exchange - A Literature Survey to December 1965," Atomic Energy of Canada Report: AECL-2546, 52 pp. (1966).

85. H. Green, "Uses of Liquid Ion-Exchangers in Inorganic Analysis," Talanta, 20, 139-161 (1973).

86. H. Hilgen, G. J. DeJong and W. L. Sederel, "Styrene-Divinylbenzene Copolymers - II. The Conservation of Porosity in Styrene-Divinylbenzene Copolymer Matrices and Derived Ion Exchange Resins," J. App1. Polymer Sclence 19, 2647-2654 (1975).

87. R. Kunin, E. Meitzner and N. Bortnick, "Macrorecticular Ion Exchange Resins," J. Am. Chem. Soc. 84, 305-306 (1962). 
88. W. L. Sederel and G. J. DeJong, "Styrene-Divinylbenzene Copolymers Construction of Porosity in Styrene Divinylbenzene Matrices," J. Appl. Polymer Science, 17, 2835-2346 (1973).

89. IUPAC - "Recommendations on Ion Exchange Nomenclature," Pure and Applied Chemistry, 29, 619-624 (1972).

90. F. C. Nachod and J. Schubert, "Ion Exchange Technology," Academic Press, Inc., New York, N. Y. Ch. 8 (1956).

91. R. H. Wiley and R. M. Dyer, "Analytical and Preparative Vapor-phase Chromatographic Separation of Divinylbenzene Mixtures," J. of Polymer Science: Part A, 2, 3153-3158 (1964).

92. R. H. Wiley and T. K. Venkatachalam, "Rates of Sulfonation of Polystyrenes Cross-1inked with Pure p-, 2:1/m:p-, and Commercial Divinylbenzene," J. of Polymer Science: Part A, 3, 1063-1067 (1965).

93. G. H. Fisher and W. D. Threadgi11, "Terminal Report on an Applied Research Program on Radiation Stability and Inorganic Radiochemistry," USAEC Report: TID-21385 (Sept. 1964).

94. I. R. Higgins, "Radiation Damage to Organic Ion Exchange Materials," USAEC Report: ORNL-1325 (Feb. 1953).

95. G. I. Cathers, "Radiation Damage to Radiochemical Processing Reagents," in Progress in Nuclear Energy, Series III, Process Chemistry, Vol. I, Pergamon Press, New York, N. Y., 68-78 (1956).

96. S. Specht, V. Dornow, W. Weinlander and J. Born, "Variation in the performance of chromatographic systems upon transition to high activities-II. Comparison of the effect of $\alpha-$ and $\delta$-radiolysis of a cation-exchange resin on distribution coefficients, separation factors and plate heights," (Translated from German), J. Radioanalytical Chemistry 26, (17-30 (1975).

97. M. Pesek and Z. Spurny, "Effect of ionizing radiation on various types of ion exchange resins based on styrene divinylbenzene," (Translated from Czech) Jaderna Energie 8(14), 313-320, (1968).

98. V. I. Paramanova, N. B. Vysokoostrovskaya, V. M. Nikolayev, S. V. Osipov and V. I. Frolov, "Effect of $\alpha$-irradiation on the characteristics of AV23 anion exchange resin, (Translated from Russian), Radiokhimiya 12(1), 127-132 (1970).

99. J. Barghusen and A. A. Jonke, "Incident at plutonium processing facility at Hanford," Reactor Fuel Processing 7(4), 297-303 (1964).

100. J. Barghusen and A. A. Jonke, "Rupture of plutonium anion exchange column as Fontenay-aux-Roses," Reactor Fue 1 Process 7(4), 303-304 (1964). 
101. J. Barghusen and A. A. Jonke, "Anion-exchange Resin Fire," Reactor Fuel Process 8(2), 135-136 (1965).

102. J. Barghusen and A. A. Jonke, "Incident involving a plutonium anionexchange contactor at Rocky Flats," Reactor Fuel Process, 8(1), 65-67 (1965).

103. J. Barghusen and A. A. Jonke, "Compatibility studies of anion-exchange resis," Reactor Fuel Process 9(2), 132-135 (1966).

104. W. J. Van Slyke, "Final Report of Chemcal Safety for Technicium-99 Recovery by Anion Exchange," BNWL-476, Battelle Pacific Northwest Laboratories, Richland, Washington, (Jan. 1967).

105. W. J. Van Slyke, G. Jansen Jr, and W. H. Swift, "Thermal Effects in Anion Exchange Resin-nitric Acid Systems," BNWL-114, Battelle Northwest (Aug. 1965).

106. F. W. Miles, "Ion Exchange Resin System Failures in Reprocessing Activities," Nuclear Safety 9(5), 394-406 (1968).

107. R. W. Powe11, Letter to the editor of Nuclear Safety, Nuclear Safety, 10, 84 (1969).

108. "Investigation of the Chemical Explosion of an Ion Exchange Resin Column and Resulting Americium Contamination of Personnel in the 242-Z Building, Aug. 30, 1976," ERDA, Richland Operations Office, 62 pp. (Oct. 1976). 


\section{APPENDIX I}

This material was extracted from reference number one:

E. V. Egorov and P. D. Novikov, "Action of Ionizing Radiation on IonExchange Materials," Atomizdat, Moscow (1965), Israel Program for Scientific Translations, Jerusalem (1967),

Appendix I, pages 230-239 with the permission of the publisher. This Appendix contains useful information of the chemical composition and structure of the major types of ion exchange resins. 
APPENDIX-I

BRIEF DESCRIPTION OF MAJOR TYPES OF ION-EXCHANGE MATERIALS[1]

\begin{tabular}{|c|c|c|c|c|}
\hline $\begin{array}{l}\text { Type and brand } \\
\text { of ion exchanger }\end{array}$ & Active groups & $\begin{array}{c}\text { Base } \\
\text { material }\end{array}$ & $\begin{array}{l}\text { TEC, } \\
\text { meq/g }\end{array}$ & Notes \\
\hline $\begin{array}{l}\text { Strongly acid, } \\
\text { monofunctional } \\
\text { sulfonated cation } \\
\text { excliangers based } \\
\text { on styrene-DVB } \\
\text { copolymers: } \\
\text { KU-2; SDV-3; } \\
\text { Dowex-50 and } \\
\text { 50W: Amber- } \\
\text { lite } \mathbb{R}-120 \text { and } \\
\text { IR-112; Wofa- } \\
\text { tit KPS (KPS- } \\
\text { 200); Catex S; } \\
\text { Imac C-12; } \\
\text { Zeolex SA: } \\
\text { Dienon SK-1 } \\
\text { and SK-100; } \\
\text { Nalcite HCR: } \\
\text { Permutit } Q\end{array}$ & $-\mathrm{SO}_{3} \mathrm{H}$ & $\begin{array}{l}\text { Styrene, } \\
\text { DVB }\end{array}$ & $4.5-5$ & $\begin{array}{l}\text { Cation ex- } \\
\text { changers } \\
\text { differ from } \\
\text { one another } \\
\text { in their } \\
\text { DVB content, } \\
\text { copolymer- } \\
\text { ization } \\
\text { techniques } \\
\text { employed, } \\
\text { conditions of } \\
\text { sulfonation, } \\
\text { etc. }\end{array}$ \\
\hline $\begin{array}{l}\text { Strongly acid, } \\
\text { monofunctional } \\
\text { sulfonated cation } \\
\text { exchangers based } \\
\text { on styrene-buta- } \\
\text { diene copoly- } \\
\text { mers: } \\
\text { SBS-1 and SBS-2 }\end{array}$ & $-\mathrm{SO}_{3} \mathrm{H}$ & $\begin{array}{l}\text { Styrene. } \\
\text { buta- } \\
\text { diene }\end{array}$ & $2.3-4$ & $\begin{array}{l}\text { SBS-1 and } \\
\text { SBS-2 differ } \\
\text { from each } \\
\text { other in their } \\
\text { content of } \\
\text { styrene and } \\
\text { butadiene. } \\
\text { Tridimension- } \\
\text { al structure } \\
\text { produced by } \\
\text { formation of } \\
\text { sulfone } \\
\text { bridges }\end{array}$ \\
\hline $\begin{array}{l}\text { Strongly acid, } \\
\text { polyfunctional } \\
\text { sulfonated cation } \\
\text { exchangers based } \\
\text { on phenol-form- } \\
\text { aldehyde resins: } \\
\text { KU-1: KU-1g: } \\
\text { Wofatit F: F } \mathrm{F}_{8} \text { : } \\
\mathrm{F}_{4} \mathrm{~S}_{\mathrm{F}} \mathrm{FF}\end{array}$ & $\begin{array}{c}-\mathrm{SO}_{\mathbf{y}} \mathrm{H} \\
-\mathrm{OH}\end{array}$ & $\begin{array}{l}\text { Phenol. } \\
\text { formal- } \\
\text { dehyde }\end{array}$ & $4.5-6$ & $\begin{array}{l}\text { KU-1g (granu- } \\
\text { lated) has } \\
\text { better hydro- } \\
\text { dynamic } \\
\text { character- } \\
\text { istic than } \\
\text { KU-1. Sul- } \\
\text { fone groups } \\
\text { may a bo be } \\
\text { located in } \\
\text { the ortho- } \\
\text { position }\end{array}$ \\
\hline
\end{tabular}


APPENDIX-I (Continued)

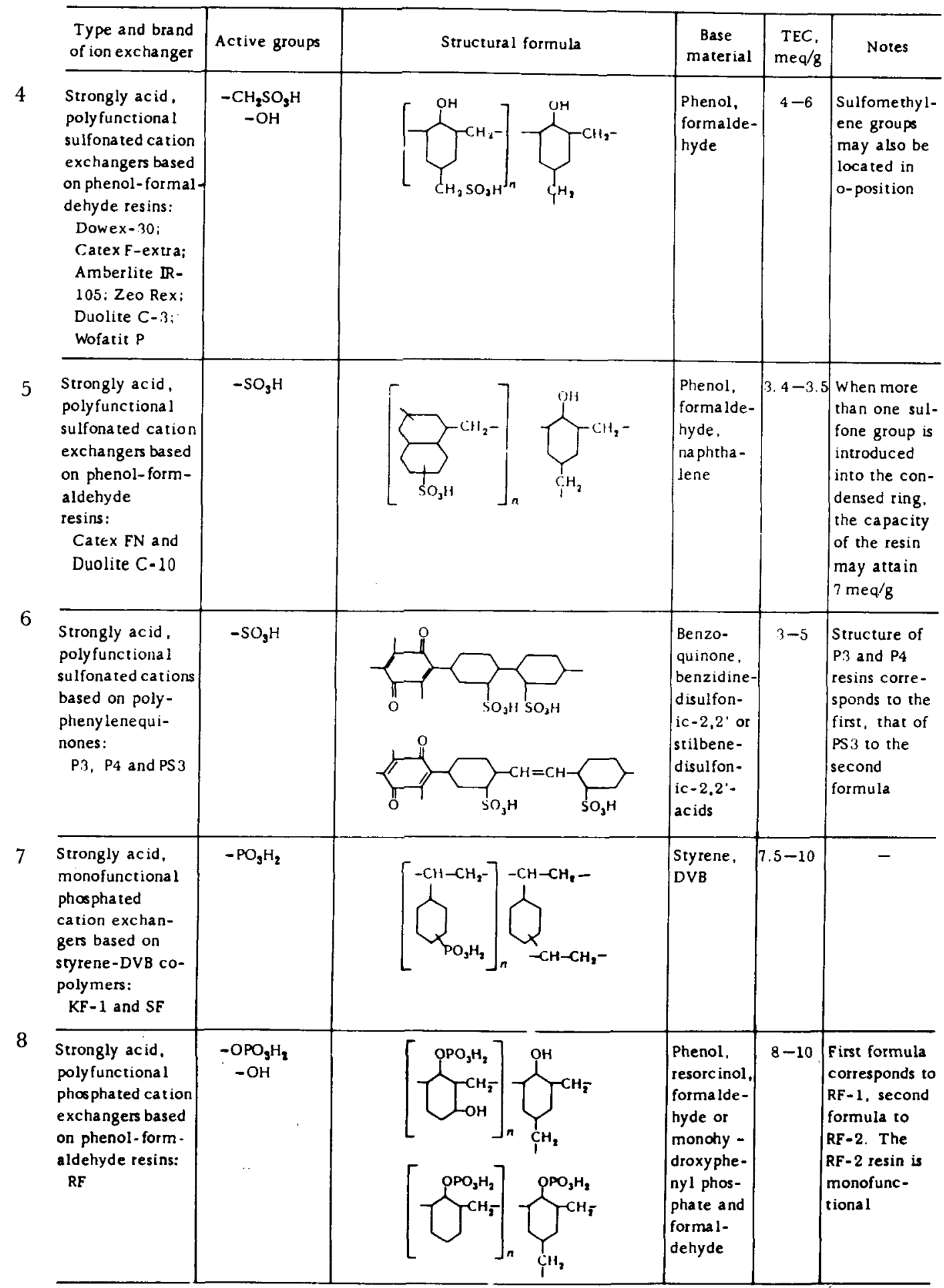


APPENDIX-I (Continued)

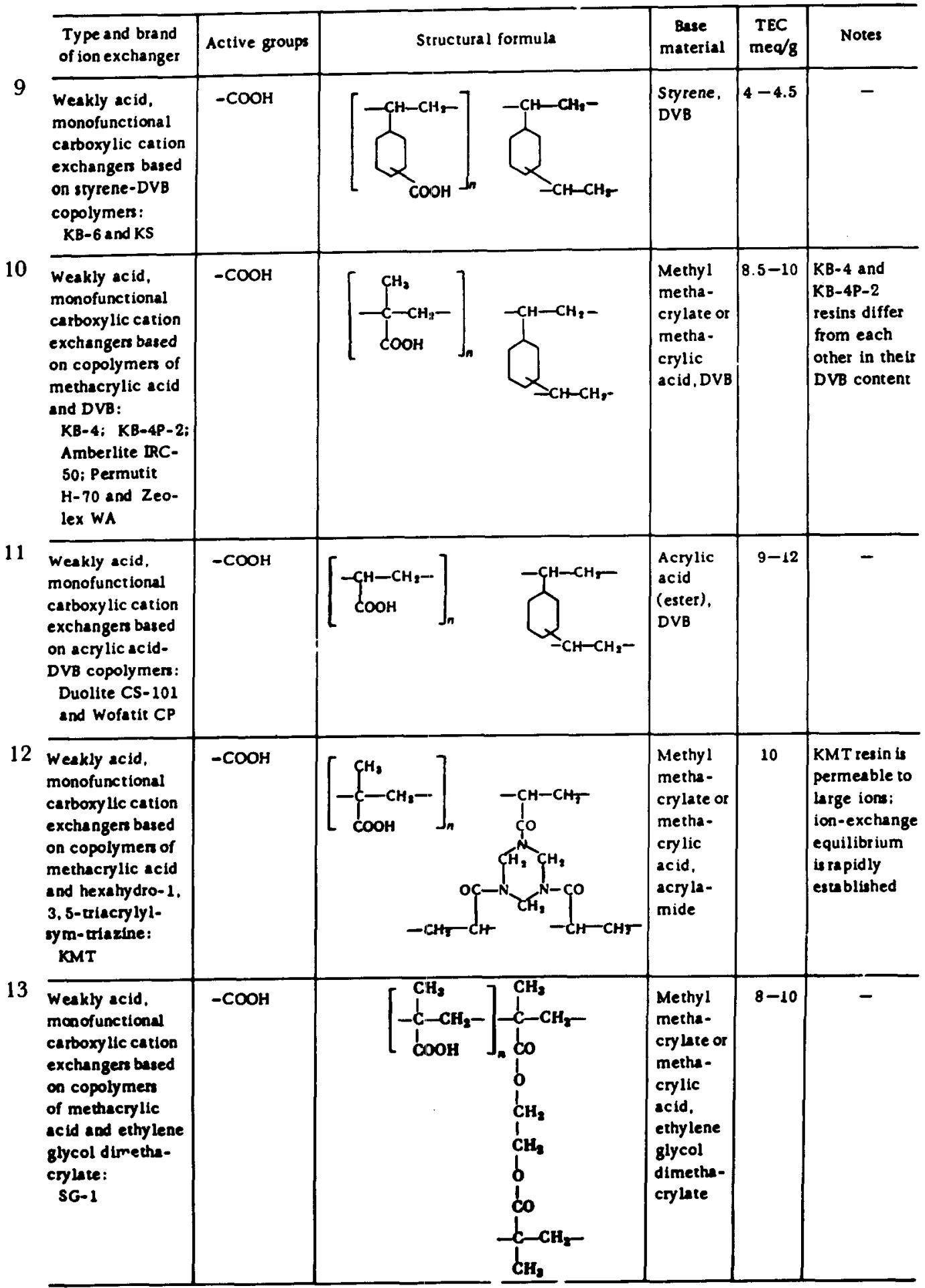


APPENDIX-I (Continued)

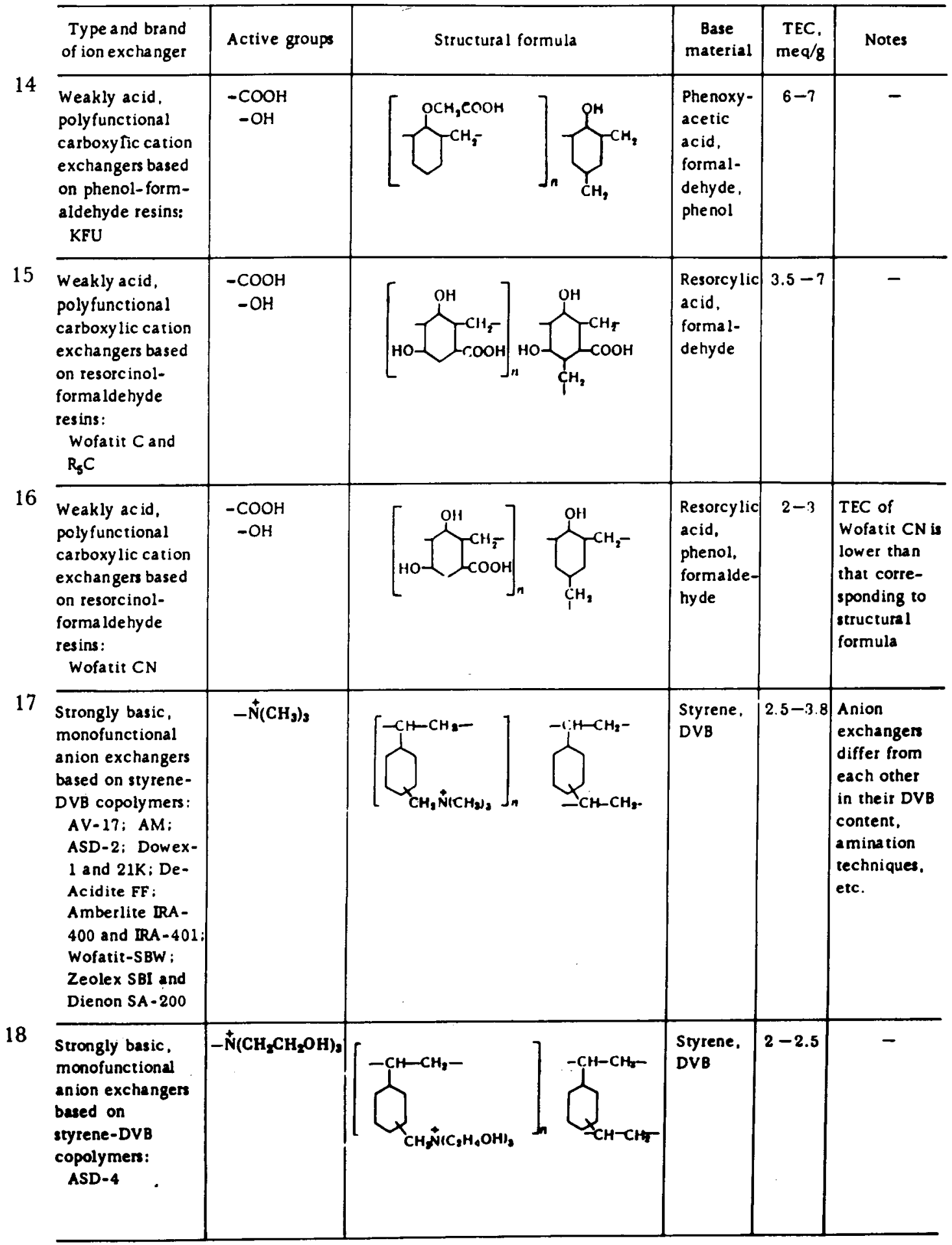


APPENDIX-I (Continued)

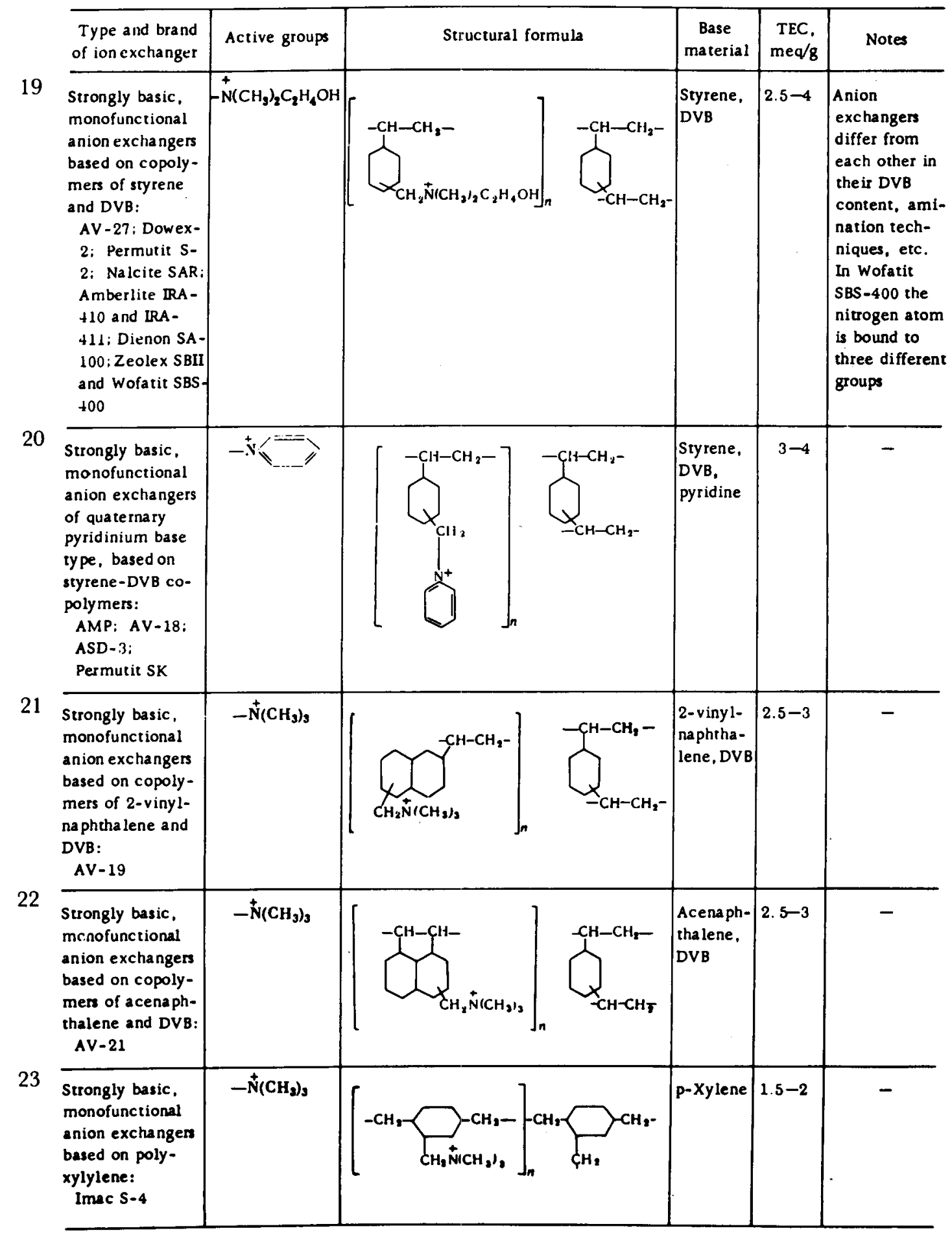


APPENDIX-I (Continued)

\begin{tabular}{|c|c|c|c|c|c|}
\hline $\begin{array}{l}\text { Type and brand } \\
\text { of ion exchanger }\end{array}$ & Active groupi & Structural formula & $\begin{array}{c}\text { Base } \\
\text { material }\end{array}$ & $\begin{array}{l}\text { TEC, } \\
\text { med } \sqrt{8}\end{array}$ & Notes \\
\hline $\begin{array}{l}\text { Strongly basic, } \\
\text { monofunctional } \\
\text { anion exchangers } \\
\text { based on triani- } \\
\text { sole sulfonium- } \\
\text { forma ldehyde } \\
\text { resins: } \\
\text { Stamex S; } \\
\text { (Stamex S-44) }\end{array}$ & $=\stackrel{+}{s}$ & $\int_{-0}^{C_{1} H_{2}}$ & $\begin{array}{l}\text { Anisole, } \\
\text { formalde- } \\
\text { hyde }\end{array}$ & $1.5-2$ & - \\
\hline $\begin{array}{l}\text { Polyfunctional } \\
\text { anion exchangers } \\
\text { of the condensa- } \\
\text { tion type with } \\
\text { quaternary pyri- } \\
\text { dinium base } \\
\text { groups: } \\
\text { AV-16 }\end{array}$ & $\begin{array}{l}=\mathbf{N} \\
=\mathbf{N H}\end{array}$ & 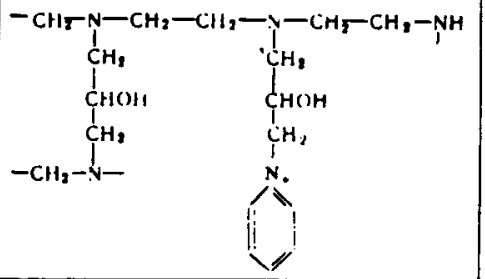 & $\begin{array}{l}\text { Pyridine, } \\
\text { polyethy- } \\
\text { lente poly- } \\
\text { amines. } \\
\text { epichoro- } \\
\text { hydrin }\end{array}$ & $\begin{array}{l}8.5- \\
10.5\end{array}$ & $\begin{array}{l}\text { AV-16 } \\
\text { conta ins } 25- \\
20 \% \text { pyri- } \\
\text { dinium groups } \\
\text { and } 75-80 \% \\
\text { secondary } \\
\text { and tertiary } \\
\text { amino group }\end{array}$ \\
\hline $\begin{array}{l}\text { Polyfunctional } \\
\text { anion exchangers } \\
\text { of the condensa- } \\
\text { tion type: } \\
\text { EDE-10: EDE- } \\
\text { 10P: Wofatit } \\
\text { L(L-150): } \\
\text { Amberlite XE- } 114\end{array}$ & $\begin{array}{l}\equiv \stackrel{+}{N} \\
\equiv N \\
=N \mathbf{N H}\end{array}$ & 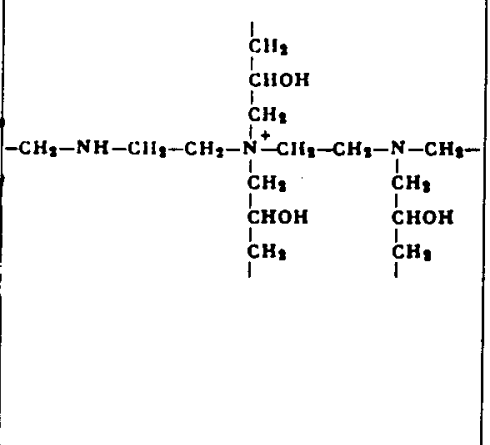 & $\begin{array}{l}\text { Polyethy- } \\
\text { lene poly- } \\
\text { amines. } \\
\text { epichloro- } \\
\text { hydrin }\end{array}$ & $8-9.5$ & $\begin{array}{l}\text { EDE- } 10 \mathrm{P} \text { may } \\
\text { be considered } \\
\text { as either } \\
\text { medium or } \\
\text { weakly basic } \\
\text { anion exchan- } \\
\text { gers. EDE-10 } \\
\text { contains a } \\
\text { smallet } \\
\text { amount of } \\
\text { polyethylene } \\
\text { polyamines } \\
\text { than EDE-1OP }\end{array}$ \\
\hline $\begin{array}{l}\text { Weakly basic, } \\
\text { monofunctional } \\
\text { pyridine-con- } \\
\text { taining. anion } \\
\text { exchangers based } \\
\text { on copolymers } \\
\text { of } 2 \text {-methyl-5- } \\
\text { vinylpyridine and } \\
\text { DVB: } \\
\text { VP-1 }\end{array}$ & & {$[-\mathrm{CH}-$} & $\begin{array}{l}\text { 2-methyl- } \\
\text { 5-vinyl- } \\
\text { pyridine. } \\
\text { DVB }\end{array}$ & $6-7$ & $\begin{array}{l}\text { VP-1 is } \\
\text { analogous to } \\
A N-25\end{array}$ \\
\hline $\begin{array}{l}\text { Weakly basic, } \\
\text { mon ofunctional } \\
\text { anion exchangens } \\
\text { based on styrene- } \\
\text { DVB copolymers: } \\
\text { AN-24 }\end{array}$ & $\begin{array}{l}\mathrm{H}_{2} \mathrm{C} \\
\mathrm{H}_{2} \mathrm{C}\end{array}$ & $-\mathrm{CH}_{2}-$ & $\begin{array}{l}\text { Styrene. } \\
\text { DVB }\end{array}$ & $4-4.5$ & $\begin{array}{l}\text { AN-24 may } \\
\text { also be } \\
\text { considered } \\
\text { as med lum- } \\
\text { basic anion } \\
\text { exchanger }\end{array}$ \\
\hline
\end{tabular}


APPENDIX-I (Continued)

\begin{tabular}{|c|c|c|c|c|c|}
\hline $\begin{array}{l}\text { Type and brand } \\
\text { of ion exchanger }\end{array}$ & Active groups & Structural formula & $\begin{array}{l}\text { Base } \\
\text { material }\end{array}$ & $\begin{array}{c}\text { TEC, } \\
\mathrm{meq} / \mathrm{g}\end{array}$ & Notes \\
\hline $\begin{array}{l}\text { Weakly basic, } \\
\text { polyfunctional } \\
\text { a nion exchangers } \\
\text { based on styrene- } \\
\text { DVB copolymers: } \\
\text { Nalcite WBR: } \\
\text { Dowex-3; } \\
\text { Amberlite TR-45: } \\
\text { Zeolex WB }\end{array}$ & $\begin{array}{l}\equiv \mathbf{N} \\
=\mathbf{N H} \\
-\mathbf{N H}_{2}\end{array}$ & {$[\underbrace{\mathrm{C}_{-\mathrm{CH}-\mathrm{CH}_{2}-}^{\mathrm{CH}-\mathrm{CH}_{2}}}_{\mathrm{CH}_{2}{ }_{\mathrm{R}_{3}}^{-\mathrm{CH}-\mathrm{CH}_{2}-}}$} & $\begin{array}{l}\text { Styrene. } \\
\text { DVB }\end{array}$ & .3.5-5.5 & $\begin{array}{l}\text { In anion ex- } \\
\text { changers of } \\
\text { this type, } \mathrm{R}_{1} \\
\text { and } \mathrm{R}_{2} \mathrm{can} \text { be } \\
-\mathrm{CH}_{2},-\mathrm{CH}_{3} \\
-\mathrm{C}_{2} \mathrm{H}_{5} \text {. The } \\
\text { degree of } \\
\text { cross-linking } \\
\text { is increased } \\
\text { by methylene } \\
\text { bridges link- } \\
\text { ing two } \\
\text { nitrogen } \\
\text { atoms }\end{array}$ \\
\hline $\begin{array}{l}\text { Weakly basic, } \\
\text { polyfunctional } \\
\text { condersation type } \\
\text { anion exchangers: } \\
\text { MMG-1 }\end{array}$ & $\begin{array}{l}\equiv \mathbf{N} \\
=\mathbf{N H}\end{array}$ & $\begin{array}{c}\text { Data on chemical structure not } \\
\text { available }\end{array}$ & $\begin{array}{l}\text { Urea, } \\
\text { melamine. } \\
\text { guanidine. } \\
\text { formal- } \\
\text { dehyde }\end{array}$ & $3.5-4.5$ & $\begin{array}{l}\text { MMG-1 is } \\
\text { sometimes } \\
\text { classified as } \\
\text { a medium- } \\
\text { basic anion } \\
\text { exchanger }\end{array}$ \\
\hline $\begin{array}{l}\text { Weakly acid, } \\
\text { polyfunctional } \\
\text { a nion exchangers } \\
\text { of the condensa- } \\
\text { tion type, based } \\
\text { on melamine: } \\
\text { AN-1 }\end{array}$ & $\begin{array}{l}=\mathbf{N H} \\
-\mathrm{NH}_{2}\end{array}$ & 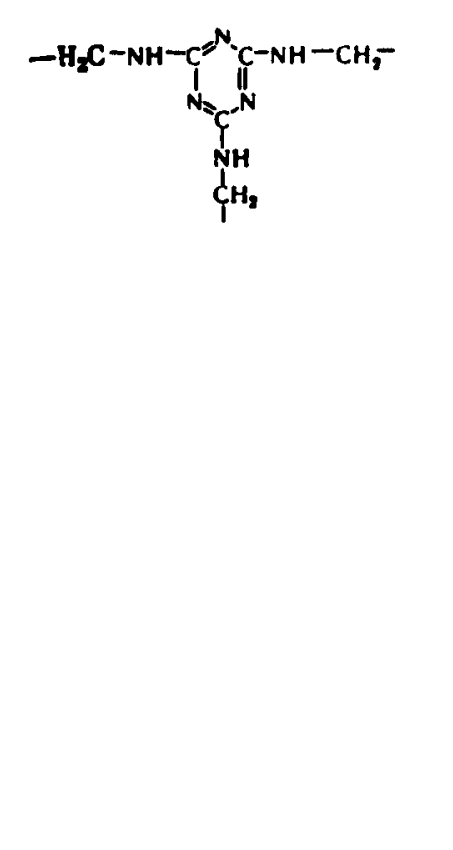 & $\begin{array}{l}\text { Melamine } \\
\text { forma l- } \\
\text { dehyde }\end{array}$ & $4-5$ & $\begin{array}{l}\text { Since not all } \\
\text { the amino } \\
\text { groups of the } \\
\text { melamine } \\
\text { participate in } \\
\text { the conden- } \\
\text { sation reac- } \\
\text { tion, the } \\
\text { exchanger } \\
\text { contains a } \\
\text { certain } \\
\text { amount of } \\
\text { primary } \\
\text { amino groups. } \\
\text { AN- } 1 \text { may } \\
2 \text { lso be ob- } \\
\text { tained by the } \\
\text { condensation } \\
\text { of trimethyl: } \\
\text { olmelamine } \\
\text { in acid } \\
\text { medium }\end{array}$ \\
\hline
\end{tabular}


APPENDIX-I (Continued)

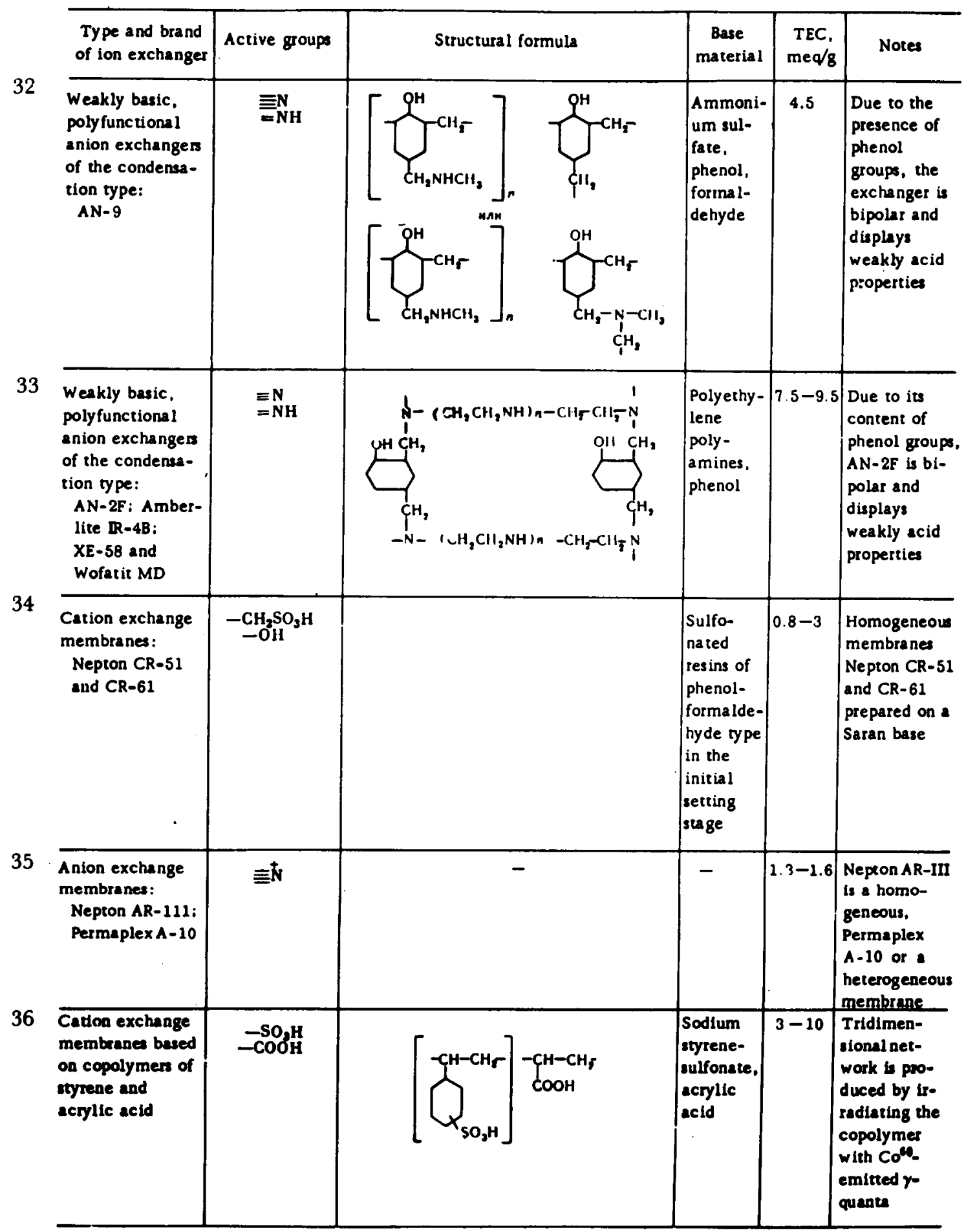


APPENDIX-I (Continued)

\begin{tabular}{|c|c|c|c|c|c|}
\hline $\begin{array}{l}\text { Type and brand } \\
\text { of ton exchanger }\end{array}$ & Active groups & Structural formula & $\begin{array}{c}\text { Base } \\
\text { material }\end{array}$ & $\begin{array}{l}\text { TEC, } \\
\text { meq'g }\end{array}$ & Notes \\
\hline $\begin{array}{l}\text { Ion-exchange } \\
\text { membra nes based } \\
\text { on graft copoly- } \\
\text { mers }\end{array}$ & $\begin{array}{l}-\mathrm{SO}_{3} \mathrm{H} \\
-\mathrm{PO}_{3} \mathrm{H}_{2} \\
-\mathrm{COOH}^{\prime} \\
-\mathrm{N}\left(\mathrm{CH}_{3}\right)_{3}\end{array}$ & 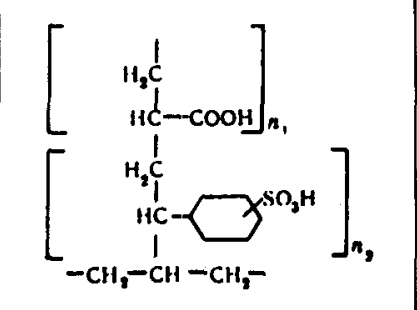 & $\begin{array}{l}\text { Polyolefin } \\
\text { membranes, } \\
\text { styrene, } \\
\text { acrylic acid, } \\
\text { vinyl ace- } \\
\text { tate, acryl- } \\
\text { onitrile }\end{array}$ & $4-5$ & $\begin{array}{l}\text { Nembranes pre- } \\
\text { pared by radiation } \\
\text { graft polymeri- } \\
\text { zation of the } \\
\text { monomers onto } \\
\text { various polyole- } \\
\text { finic (ma inly } \\
\text { polyethylene) } \\
\text { membranes }\end{array}$ \\
\hline $\begin{array}{l}\text { Coal-based poly- } \\
\text { functional sulfona- } \\
\text { ted cation exchan- } \\
\text { gers: } \\
\text { sulfonated coals. } \\
\text { Dusarit S }\end{array}$ & $\begin{array}{c}-\mathrm{SO}_{\mathrm{H}}^{\mathrm{H}} \\
-\mathrm{CoO} \mathrm{H} \\
-\mathrm{OH}\end{array}$ & $\begin{array}{l}\text { Sorbents are frequently synthesized } \\
\text { from activated coals consisting } \\
\text { of complex aggregates formed } \\
\text { from crystalline carbon with a } \\
\text { graphite lattice }\end{array}$ & $\begin{array}{l}\text { Black coal, } \\
\text { brown coal } \\
\text { and charcoal }\end{array}$ & $0.8-3.6$ & $\begin{array}{l}\text { The various sul- } \\
\text { fonated coals } \\
\text { differ from eacli } \\
\text { otlier in the iden- } \\
\text { tity of the raw } \\
\text { material and sul- } \\
\text { fonation tecli- } \\
\text { niques }\end{array}$ \\
\hline $\begin{array}{l}\text { Polyfunctional } \\
\text { weakly acid cation } \\
\text { exchangers based } \\
\text { on oxidized coals: } \\
\text { lump coal: BAU: } \\
\text { KAU: SKT and } \\
\text { SKLT }\end{array}$ & $\begin{array}{l}-\mathrm{COOH} \\
-\mathrm{OH}\end{array}$ & - & \begin{tabular}{|l} 
Black coal \\
and charcoal
\end{tabular} & 4 & $\begin{array}{l}\text { Capacity of oxi- } \\
\text { dized coa is } \\
\text { strongly depends } \\
\text { on tlie pll of the } \\
\text { solution }\end{array}$ \\
\hline $\begin{array}{l}\text { Polyfunctional, } \\
\text { weakly basic anion } \\
\text { exchangers based } \\
\text { on coals: } \\
\text { BAU: KAU, SKT } \\
\text { and SKLT }\end{array}$ & & - & The same & $0.35-0.5$ & $\begin{array}{l}\text { The exchanger is } \\
\text { obtained by } \\
\text { treating active } \\
\text { (nonoxidized) } \\
\text { cosls with HF and } \\
\mathrm{HCl} \text {. In a number } \\
\text { of cases the TEC } \\
\text { of anion-ex- } \\
\text { changer coals may } \\
\text { be as high as } 2- \\
3 \text { meq } / 8\end{array}$ \\
\hline $\begin{array}{l}\text { Inorganic cation } \\
\text { exchangers of } \\
\text { mineral origin: } \\
\text { kaolinite }\end{array}$ & $-\mathrm{OH}$ & $\mathrm{Al}_{4}\left[\mathrm{Si}_{4} \mathrm{O}_{80}\right] \mid \mathrm{OH}_{\mathbf{s}}$ & $\begin{array}{l}\text { Kaolinite } \\
\text { group } \\
\text { miners is }\end{array}$ & $\begin{array}{l}0.02- \\
0.05(\text { in } \\
\text { neutral } \\
\text { solu- } \\
\text { tions) }\end{array}$ & $\begin{array}{l}\text { Sorbent is } \\
\text { unstable in alka- } \\
\text { line solutions. It } \\
\text { is sometimes used } \\
\text { compounded with } \\
\text { binder (cellulose) }\end{array}$ \\
\hline $\begin{array}{l}\text { Inorganic cation } \\
\text { exchangers based } \\
\text { on hydrated } \\
\text { silicic acid: } \\
\text { sllica gel }\end{array}$ & $-\mathrm{OH}$ & $\begin{array}{c}\mathrm{CH} \\
-\mathrm{OH} \\
-\mathrm{O} i-0-\mathrm{Si}-0-\end{array}$ & $\begin{array}{l}\text { Hydtogel of } \\
\text { silicic acid }\end{array}$ & $\begin{array}{l}10^{-3}- \\
10^{-8} \text { (in } \\
\text { neutral } \\
\text { solu- } \\
\text { tion) }\end{array}$ & $\begin{array}{l}\text { Sorbent is } \\
\text { unstable in } \\
\text { alksline solutions }\end{array}$ \\
\hline
\end{tabular}


APPENDIX-I (Continued)

43

Type and brand of ion exchanger Inorganic cation exchangers based on alumosilicates:

Permutit $G$

Inorganic cation exchangers based on metal oxides and hydroxides: manganese dioxide

oxide

Inorganic cation exchangers based on zirconium: zirconium phosphate (zirconyl phosphate.

zirconium polyphosphate)

\begin{tabular}{l} 
phosphate) \\
\\
\hline Inorganic cation \\
exchangers based \\
on tin: \\
tin phosphate \\
(tin polyphos- \\
phate)
\end{tabular}

\begin{tabular}{|l|l|}
\hline Active groups & \\
\hline &
\end{tabular}

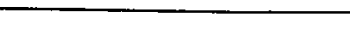

Structural formula

mat




\section{APPENDIX II}

This material was extracted from reference number two:

I.A.E.A. Technical Report Series No. 78, "Operation and Control of Ionexchange Processes for Treatment of Radioactive Wastes," International Atomic Energy Agency, Vienna (1967),

Tables X through XII on pages 108 to 127 with the permission of the publisher.

This appendix is a listing of the general physical properties of ion exchange resins useful to nuclear waste separations. 
APPENDIX-II

GENERAL PROPERTIES OF ION EXCHANGE MATERIALS[2]

(From Manufacturers' Brochures)

SYNTHETIC CATION EXCHANGERS

a. Polystyrene base, bead form addition polymers

\begin{tabular}{|c|c|c|c|c|c|c|c|c|c|c|}
\hline \multirow[b]{2}{*}{ Trade areme } & \multirow[b]{2}{*}{ Manulecturer } & \multirow[b]{2}{*}{$\begin{array}{l}\text { Fumctional } \\
\text { group }\end{array}$} & \multirow[b]{2}{*}{ lon lorm } & \multicolumn{2}{|c|}{ Exchange capecity } & \multicolumn{2}{|c|}{ Physical chanecteriseica } & \multicolumn{2}{|c|}{ Operational conerols } & \multirow[b]{2}{*}{ Remarts } \\
\hline & & & & $\begin{array}{l}\text { (med/g) } \\
\text { (dry) }\end{array}$ & $\begin{array}{l}\text { (med/ml) } \\
\text { (wet) }\end{array}$ & $\begin{array}{l}\text { Molzure } \\
\text { (M.x) }\end{array}$ & $\begin{array}{l}\text { size } \\
(\mathrm{mm})\end{array}$ & $\begin{array}{l}\text { Max. oper. } \\
\text { remp. ("C) }\end{array}$ & 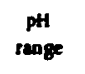 & \\
\hline Allentoo CS & Proalm, Fresce & - $\mathrm{soi}^{\circ}$ & & & & & $0.3-0.6$ & 220 & $1 \cdot 14$ & \\
\hline 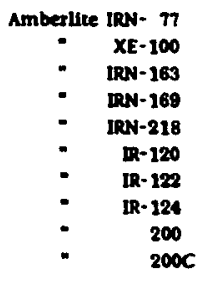 & 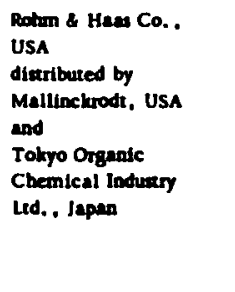 & 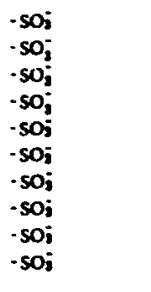 & 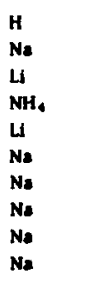 & $\begin{array}{l}4.7 \\
\therefore .5 \\
4.6 \\
4.4 \\
4.6 \\
4.3-5 \\
4.3-5 \\
4.3-5 \\
4.3 \\
4.3\end{array}$ & $\begin{array}{l}1.9 \\
2.1 \\
2.2 \\
1.75 \\
1.75\end{array}$ & $\begin{array}{l}55 \\
56-60 \\
55 \\
55 \\
55 \\
45-48 \\
39-43 \\
37-41 \\
46-51 \\
46-51\end{array}$ & $\begin{array}{l}0.3-1.2 \\
0.3 \cdot 1.2 \\
0.3-1.2 \\
0.3-1.2 \\
0.3-1.2 \\
0.3-1.2 \\
0.3-1.2 \\
0.3-1.2\end{array}$ & $\begin{array}{l}120 \\
150 \\
150 \\
150 \\
120 \\
120 \\
120 \\
120 \\
120\end{array}$ & $\begin{array}{l}0.14 \\
0-14 \\
0-14 \\
0.14 \\
0-14\end{array}$ & 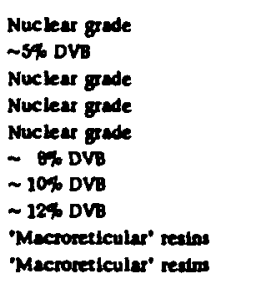 \\
\hline $\begin{array}{l}\text { Amberilyz } 15 \\
\text { (Amberiyx resima are }\end{array}$ & d lor non-aqueous appliter & $\begin{array}{l}-50 \mathrm{i} \\
\text { (100m) }\end{array}$ & & 4.8 & 1.2 & 60.68 & & 120 & 0.14 & 'Macrorteticular' realns \\
\hline 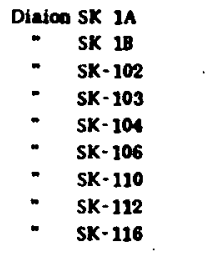 & 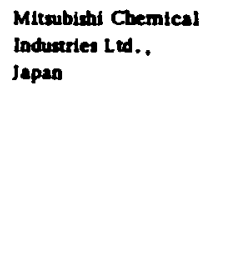 & 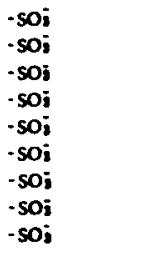 & $\begin{array}{l}\mathrm{Na} \\
\mathrm{Na} \\
\mathrm{N}_{2} \\
\mathrm{Na} \\
\mathrm{Na} \\
\mathrm{Na} \\
\mathrm{Na} \\
\mathrm{Na} \\
\mathrm{Na} \\
\mathrm{Na}\end{array}$ & $\begin{array}{l}: \\
: \\
: \\
:\end{array}$ & $\begin{array}{l}1.9 \\
1.9 \\
0.6 \\
0.9 \\
1.2 \\
1.6 \\
2.0 \\
2.1 \\
2.1\end{array}$ & $\begin{array}{l}43 \cdot 50 \\
43 \cdot 50 \\
72 \cdot 82 \\
64 \cdot 74 \\
57-67 \\
47 \cdot 57 \\
35 \cdot 45 \\
32-42 \\
27-37\end{array}$ & $\begin{array}{l}0.3-1.2 \\
0.3-1.2 \\
0.3-1.2 \\
0.3-1.2 \\
0.3-1.2 \\
0.3-1.2 \\
0.3-1.2 \\
0.3-1.2 \\
0.3-1.2\end{array}$ & $\begin{array}{l}120 \\
120 \\
120 \\
120 \\
120 \\
120 \\
120 \\
120 \\
120\end{array}$ & $\begin{array}{l}0.24 \\
0-14 \\
0-14 \\
0-14 \\
0-14 \\
0-14 \\
0.14 \\
0-14 \\
0-14\end{array}$ & 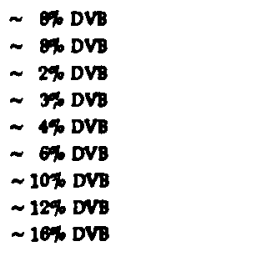 \\
\hline $\begin{array}{c}\text { Dowex so-x8 } \\
=\text { sow }-x 4 \\
.\end{array}$ & $\begin{array}{l}\text { Dow Chemical Co.. } \\
\text { USA } \\
\text { marketed by }\end{array}$ & $\begin{array}{l}-\mathrm{sO}_{3} \\
-\mathrm{sO}_{3}\end{array}$ & $\begin{array}{l}\mathrm{H} \\
\mathrm{H}\end{array}$ & $\begin{array}{l}\mathbf{5 . 0} \\
\mathbf{5 . 0}\end{array}$ & $\begin{array}{l}1.7 \\
1.7\end{array}$ & $\begin{array}{l}53 \\
53\end{array}$ & $\begin{array}{l}0.3 \cdot 0.8 \\
0.3-0.8\end{array}$ & $\begin{array}{l}150 \\
15 c\end{array}$ & & 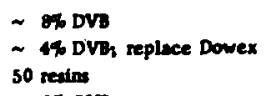 \\
\hline 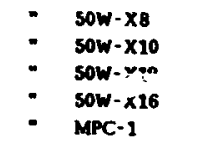 & $\begin{array}{l}\text { Naboo Chemical Co., } \\
\text { USA }\end{array}$ & $\begin{array}{l}-\mathrm{soj} \\
-\mathrm{soj} \\
-\mathrm{soi} \\
-\mathrm{SO} i \\
-\mathrm{So} ;\end{array}$ & $\begin{array}{l}\mathrm{Na} \\
\mathrm{H} \\
\mathrm{H} \\
\mathrm{H} \\
\mathrm{H}\end{array}$ & $\begin{array}{l}4.8 \\
\vdots \\
4.5-4.9\end{array}$ & $\begin{array}{l}1.9 \\
1.9 \\
2.1 \\
2.3 \\
1.8-1.8\end{array}$ & $\begin{array}{l}\begin{array}{l}42-48 \\
46-52 \\
42-48 \\
36-42\end{array} \\
3\end{array}$ & $\begin{array}{l}0.3-0.8 \\
0.3-0.8 \\
0.3-0.8 \\
0.3-0.8 \\
0.4-1.2\end{array}$ & $\begin{array}{l}150 \\
150 \\
150 \\
150 \\
300\end{array}$ & & 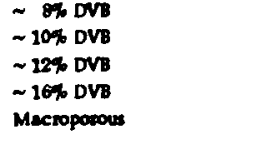 \\
\hline
\end{tabular}


APPENDIX-II (Continued)

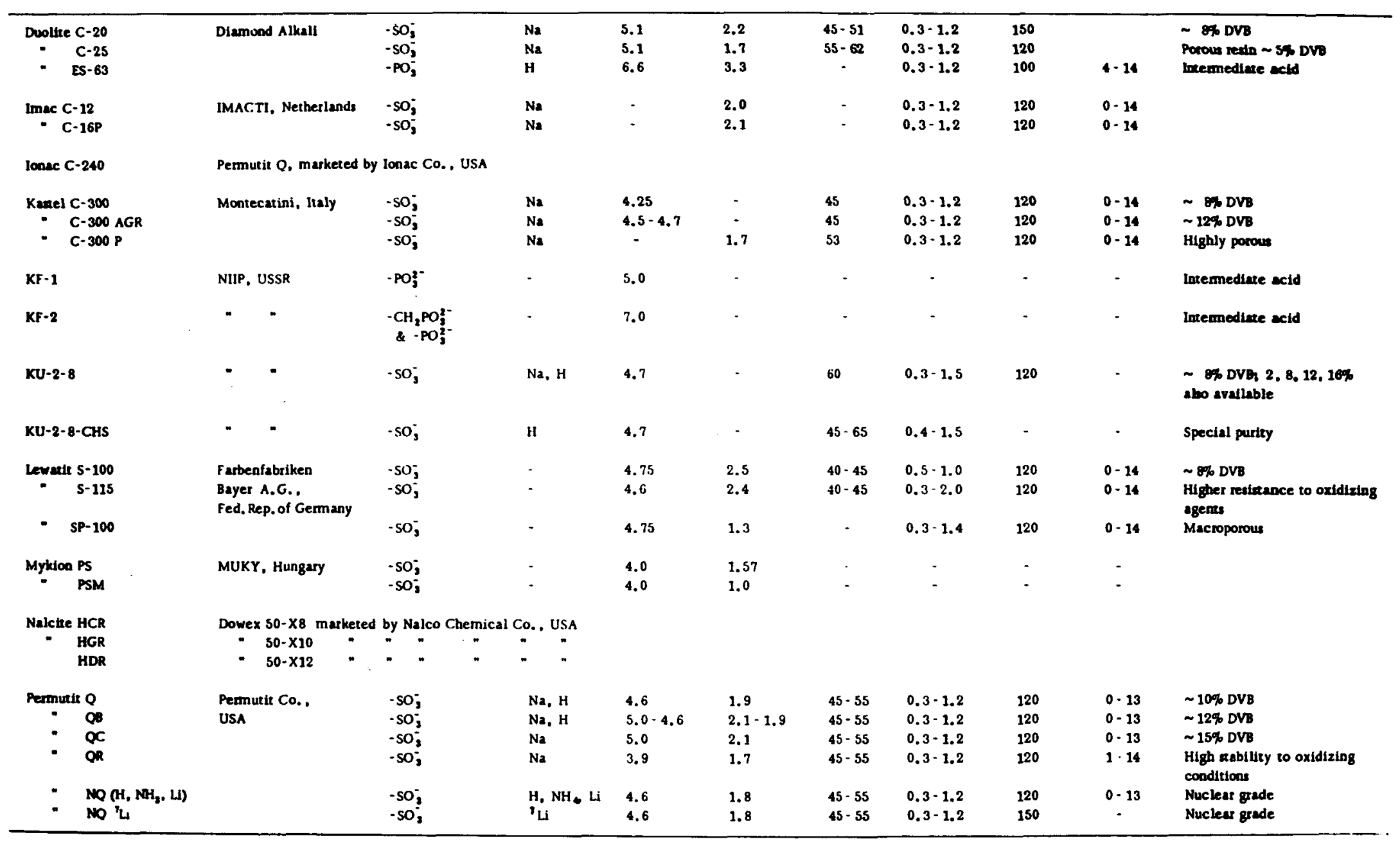


APPENDIX-II (Continued)

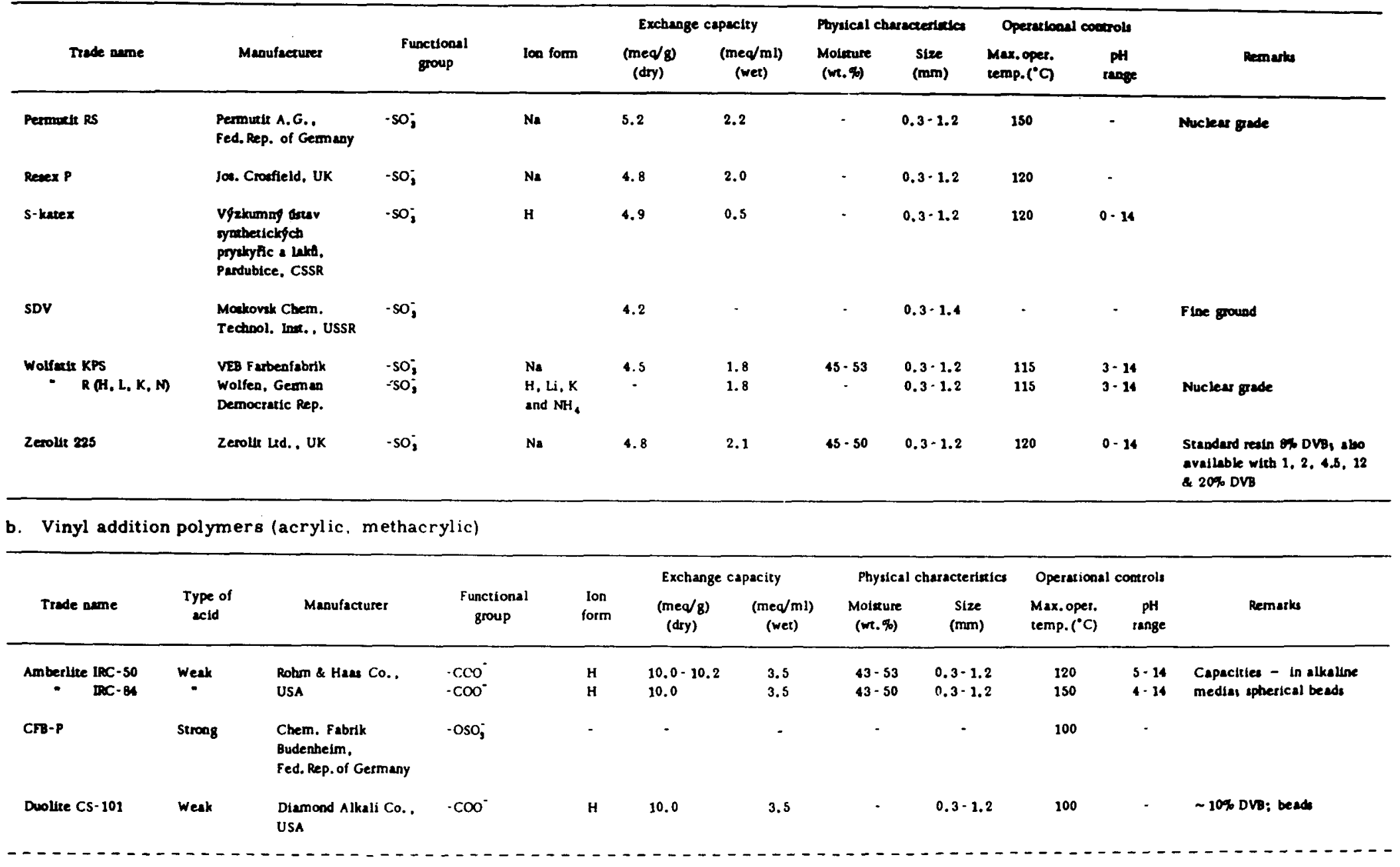


APPENDIX-II (Continued)

\begin{tabular}{|c|c|c|c|c|c|c|c|c|c|c|c|}
\hline Ionac C-270 & Permutit $\mathrm{H}^{-}$ & marketed by lonac Co.. & USA & & & & & & & & \\
\hline Imec ZS & Weak & IMACTI, Netheriands & $-\cos$ & $H$ & - & 3.0 & $\cdot$ & $0.3 \cdot 1.2$ & 100 & $4-14$ & Beads \\
\hline Kastel C-100 & Weak & Montecatini, Italy & $-\mathrm{COO}^{-}$ & H & $\cdot$ & 3.0 & $45-50$ & $0.3 \cdot 1.2$ & 110 & 0.14 & Beads: ～10\% DVB \\
\hline Karboxylovy & weak & $\begin{array}{l}\text { Spolek pro chemickou } \\
\text { výrobu, CSSR }\end{array}$ & $-\mathrm{COO}^{\circ}$ & $\mathrm{H}$ & 10.0 & 3.5 & - & $0.3 \cdot 1.2$ & 120 & $5-14$ & Beads \\
\hline KB-1 & Weak & NIIP, USSR & $-\mathrm{COO}^{-}$ & - & 10.0 & - & - & $0.3-1.5$ & - & - & Beach \\
\hline KB-2 & $"$ & " $\quad "$ & $-\cos 0^{\circ}$ & - & $10-11$ & - & - & $0.3 \cdot 1.0$ & - & - & Beads \\
\hline KB-3 & " & $" \quad \cdots$ & $-c 00^{\circ}$ & $\cdot$ & $i-i$ & - & - & $0.3-1.5$ & - & - & Beads; scrylonitrile-DVB \\
\hline$K B-4 P-2$ & $"$ & " $\quad "$ & $-\cos 0^{\circ}$ & Sa & 9.5 & $\cdot$ & & $0.25-1.0$ & - & $=$ & Déti \\
\hline $\mathrm{K} \mathbf{n}$ & Weak & $\begin{array}{l}\text { Moakovsk Chem. } \\
\text { Technol. Inex. . USSR }\end{array}$ & $-\cos )^{\circ}$ & $\cdot$ & $\dot{0} .0$ & $\cdot$ & - & $0.25-1.5$ & $\cdot$ & - & Fine ground \\
\hline $\mathrm{KF}-3$ & Intermed. & NIIP, USSR & $\cdot \mathrm{PO}_{3}^{2-}$ & $\cdot$ & 3.5 & $\cdot$ & - & $\cdot$ & $\cdot$ & $\cdot$ & \\
\hline$K f-4$ & Intermed. & 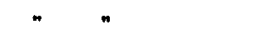 & $\mathrm{CH}_{2} \mathrm{PO}_{3}^{2-}$ & $\cdot$ & 5.5 & $\cdot$ & - & - & $\cdot$ & - & \\
\hline$K U-3$ & Strong & n $\quad$ n & $-\mathrm{SO}_{\mathbf{i}}^{-}$ & $\cdot$ & 5.5 & - & $\cdot$ & $\cdot$ & $\cdot$ & - & Fine ground \\
\hline Mykion KMK & Weak & MUKY, Hungary & $-\mathrm{COO}^{\circ}$ & - & 8.6 & 1.2 & - & - & - & - & \\
\hline$-\quad \mathrm{CP}$ & " & " $\quad$ " & $-\mathrm{COO}^{-}$ & $\cdot$ & 8.0 & 2.8 & - & $\cdot$ & - & - & \\
\hline Permutit $C$ & Weak & $\begin{array}{l}\text { Permutit A.G., } \\
\text { Fed. Rep. of Germany }\end{array}$ & $-\cos 0^{\circ}$ & $\mathrm{H}$ & 10.0 & 4.0 & - & - & 100 & $6-14$ & Beads \\
\hline$\cdot \quad H-70$ & " & Permutit Co., USA & $-\mathrm{CO} 0^{\circ}$ & $\mathbf{H}$ & 6.5 & 2.4 & $40-50$ & $0.3-1.2$ & 120 & $5-14$ & Beads \\
\hline Wolfanit CP & Weak & $\begin{array}{l}\text { VEB F arbenfabrik } \\
\text { Wolfen, German } \\
\text { Democratic Republic }\end{array}$ & $-\mathrm{COO}^{\circ}$ & $\therefore a$ & 10.0 & 3.5 & $43-53$ & $0.5-2.0$ & 100 & 7 & $\sim 5 \%$ DVB, beads \\
\hline Zerolit 226 & Weak & Zerolit Ld., , UK & $-\mathrm{COO}^{-}$ & $\mathbf{H}$ & 10.0 & 3.5 & - & $0.3-1.2$ & 100 & $\cdot$ & $\begin{array}{l}\text { Beadi; } 2.5 \& 4.5 \% \text { DVB } \\
\text { evallable }\end{array}$ \\
\hline
\end{tabular}


APPENDIX-II (Continued)

\begin{tabular}{|c|c|c|c|c|c|c|c|c|c|c|c|}
\hline \multirow[b]{2}{*}{ Trade neme } & \multirow[b]{2}{*}{$\begin{array}{l}\text { Type of } \\
\text { ecid }\end{array}$} & \multirow[b]{2}{*}{ Manufactures } & \multirow[b]{2}{*}{$\begin{array}{l}\text { Functional } \\
\text { group }\end{array}$} & \multirow[b]{2}{*}{$\begin{array}{l}\text { lon } \\
\text { form }\end{array}$} & \multicolumn{2}{|c|}{ Exchange capacity } & \multicolumn{2}{|c|}{ Physdcal characteristic } & \multicolumn{2}{|c|}{ Operational controls } & \multirow[b]{2}{*}{ Remastos } \\
\hline & & & & & $\begin{array}{l}(\text { meq/g) } \\
\text { (dry) }\end{array}$ & $\begin{array}{l}\text { (med/ml) } \\
\text { (wet) }\end{array}$ & $\begin{array}{l}\text { Molmure } \\
\text { (wt. \%) }\end{array}$ & $\begin{array}{l}\text { size } \\
\text { (mm) }\end{array}$ & $\begin{array}{l}\text { Max. oper. } \\
\text { temp. }\left({ }^{\circ} \mathrm{C}\right)\end{array}$ & $\underset{\text { range }}{\mathrm{pH}}$ & \\
\hline Dorollt fK-22 & Strong & MUKY, Hungary & $-\mathrm{SO}^{-}, \mathrm{OH}^{-}$ & $\cdot$ & 2.7 & 0.6 & - & - & - & - & Fine ground \\
\hline Dowex CCR-1 & weak & $\begin{array}{l}\text { Dow Chemical Co.. } \\
\text { USA }\end{array}$ & $\begin{array}{l}-\mathrm{COO} \\
-\mathrm{OH}\end{array}$ & H & - & 1.4 & - & $0.3-0.8$ & 100 & - & $\begin{array}{l}\text { Markered by Nalco Chem. } \\
\text { Co., USA. granular }\end{array}$ \\
\hline Duolite C-3 & Stroog & $\begin{array}{l}\text { Diamond Alkeli } \\
\text { Co., USA }\end{array}$ & - $\mathrm{sO}^{-}$ & H & 2.9 & 1.2 & - & $0,3-2.0$ & 60 & $0-9$ & Beads; $\cdot \mathrm{CH}_{2} \mathrm{SO}_{1}$ group \\
\hline$-C-10$ & $\cdot$ & - & $-\mathrm{soj}^{\circ}$ & H & 2.9 & 0.6 & - & $0.3-2.0$ & 40 & $0-9$ & Beads: $-\mathrm{CH}_{2} \mathrm{SO}_{3}^{-}$groups \\
\hline Lewatit KSN & Strong & $\begin{array}{l}\text { Farbenfabriken } \\
\text { Bayer A.G., Fed. } \\
\text { Rep. of Germany }\end{array}$ & $-\mathrm{sO}_{3}$ & - & 4.0 & 1.6 & $45-50$ & $0.3 \cdot 1.5$ & 30 & 0.8 & Granular \\
\hline - CNS & $\begin{array}{l}\text { Strong } \\
\text { \& weak }\end{array}$ & $\cdot$ & $\begin{array}{l}-\mathrm{SO} \\
-\mathrm{COO}^{-}\end{array}$ & $\bar{H}$ & 5.0 & 2.5 & $36-43$ & $0.3-1.6$ & 10 & $0-10$ & Granules \\
\hline - $\quad$ CNO & Weak & - $\quad "$ & $-\cos 0^{\circ}$ & . & 4.0 & 2.4 & $30-35$ & $0.3 \cdot 1.6$ & 40 & 0.10 & Granular \\
\hline MSF -3 & Weak & $\begin{array}{l}\text { Moskovak Chem. } \\
\text { Tech. Inst. . USSR }\end{array}$ & $\begin{array}{l}-\mathrm{SO}_{3}^{-} \\
-\mathrm{OH}\end{array}$ & - & 4.3 & $\cdot$ & - & $0.3-2.0$ & - & - & Fine ground \\
\hline $\begin{array}{c}\text { Mykion FG } \\
\text { P }\end{array}$ & Strong & MUKY, Hungary & $\begin{array}{l}-\mathrm{CH}_{2} \mathrm{SO}_{3}, \cdot \mathrm{OH} \\
-\mathrm{OH}\end{array}$ & - & $\begin{array}{l}2.8 \\
2.2\end{array}$ & $\begin{array}{l}0.9 \\
0.65\end{array}$ & : & . & . & - & \\
\hline NSF & Strong & $\begin{array}{l}\text { Moskovsk Chem. } \\
\text { Tech. Inst., USSR }\end{array}$ & $\cdot \mathrm{so}_{2}^{-}$ & $\cdot$ & 3.0 & - & - & $0.3-1.5$ & - & - & Fine ground \\
\hline Permutit H & Weak & $\begin{array}{l}\text { Permurit Co.. USA } \\
\text { Permutit A.G. . } \\
\text { Fed. Rep. of Germany }\end{array}$ & $\begin{array}{l}-c 00^{\circ} \\
-c 00^{\circ}\end{array}$ & - & $\begin{array}{l}5.0 \\
4.0\end{array}$ & 1.9 & - & $\dot{.}$ & $\begin{array}{l}65 \\
40\end{array}$ & $\dot{-}$ & $\begin{array}{l}\text { Granular } \\
\text { Granular }\end{array}$ \\
\hline Resex $W$ & Weak & Jos. Crodield, UK & $-\mathrm{COO}^{\circ}$ & - & $2.5-3.0$ & $\cdot$ & - & - & - & $\cdot$ & Granulas \\
\hline RF & Intermed. & $\begin{array}{l}\text { Moakovak Cbem. } \\
\text { Tech. Inst. . USSR }\end{array}$ & $-\mathrm{PO}_{1}^{2-}$ & - & 4.3 & - & - & - & - & - & \\
\hline
\end{tabular}


APPENDIX-II (Continued)

\begin{tabular}{|c|c|c|c|c|c|c|c|c|c|c|c|}
\hline SN & Strong & $\begin{array}{l}\text { maritur vysokomol.. } \\
\text { USSR }\end{array}$ & $\begin{array}{l}-\mathrm{SO}_{3}^{-} \\
-\mathrm{OH}\end{array}$ & - & 5.2 & - & - & $0.3-1.5$ & $\cdot$ & - & Fine ground \\
\hline SNF & Strong & $"$ & $\cdot \mathrm{SO}_{3}^{-},-\mathrm{OH}$ & $\cdot$ & 5.2 & - & - & $0.3 \cdot 1.5$ & $\cdot$ & - & Fine ground \\
\hline FN-katex & Strong & $\begin{array}{l}\text { Cbemický kombinat } \\
\text { zfluzT, CSSR }\end{array}$ & $-\mathrm{sO}_{3}^{-}$ & $\mathbf{H}$ & - & 0.54 & - & $0.3-1.5$ & - & $1-9.5$ & Beads. fine ground \\
\hline KB-5 & weak & NIIP, USSR & $-\mathrm{CH}_{2} \mathrm{COO}^{-}, \mathrm{OH}$ & & 7.5 & - & - & $0.25-1.5$ & - & - & \\
\hline KU-1 & Strong & " & $-\mathrm{SO}_{3}^{-},-\mathrm{OH}$ & - & 4.0 & - & 50 & $0.3-3.0$ & - & - & Dark brown grains \\
\hline$K U-1 G$ & Strong & $=\quad \cdot$ & $-\mathrm{SO}_{3}^{-},-\mathrm{OH}$ & - & 4.5 & - & 50 & $0.3-2.0$ & - & - & Beads, black or brown \\
\hline KRFU & Weak & $\begin{array}{l}\text { Institut vysokomol. } \\
\text { USSR }\end{array}$ & $-\mathrm{COO}^{\circ}$ & - & 4.0 & $\cdot$ & - & $0.25-1.5$ & - & - & Fine ground \\
\hline KU-5 & Strong & NIIP, USSR & $-\mathrm{sO}_{3}^{-}$ & - & 5.0 & - & - & $0.3-2.0$ & - & - & Beeds, fine ground \\
\hline $\mathrm{KU}-6$ & $\begin{array}{l}\text { Stroog } \\
\text { \& weak }\end{array}$ & - & $\begin{array}{l}-\mathrm{SO}^{-} \\
-\mathrm{COO}^{-}\end{array}$ & - & 5.5 & - & - & $\cdot$ & - & - & \\
\hline$K U-6 F$ & " & " & $-\mathrm{COO}^{-}$ & - & 5.6 & - & - & - & - & - & \\
\hline $\begin{array}{l}\mathrm{KU}-7 \\
\mathrm{KU}-\mathrm{8}\end{array}$ & . & $=\quad$ & $-\mathrm{COO}^{-}$ & - & 5.5 & - & - & - & - & - & \\
\hline $\begin{array}{l}\mathrm{KU}-8 \\
\mathrm{KU}-9\end{array}$ & Strong & $: \quad "$ & $\begin{array}{l}-\mathrm{SO}_{3},-\mathrm{COO}^{-} \\
-\mathrm{SO}_{\mathbf{3}}, \mathrm{OH}\end{array}$ & - & $\begin{array}{l}6.0 \\
6.0\end{array}$ & - & : & - & : & $\therefore$ & Fine ground \\
\hline $\mathrm{KU}-21$ & 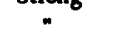 &. & $\begin{array}{l}-\mathrm{SO}_{3},-\mathrm{OH} \\
-\mathrm{SO}_{3}^{-}\end{array}$ & - & 5.5 & - & - & - & - & - & Fine ground \\
\hline Staionit f-extra & Strong & $\begin{array}{l}\text { Chemicky kombinát } \\
\text { zsluzT, CSSR }\end{array}$ & $\begin{array}{l}-\mathrm{CH}_{2} \mathrm{SO}_{3}^{-} \\
-\mathrm{OH}\end{array}$ & $\mathbf{N a}$ & - & 0.2 & & $0.5 \cdot 2.5$ & - & $1 \cdot 9.5$ & Fine ground \\
\hline Wolfatit $F$ & Strong & $\begin{array}{l}\text { VEB Farbenfabrik } \\
\text { Wolfen, Ccrman } \\
\text { Democratic Republic }\end{array}$ & $-\mathrm{sO}_{3}^{-}$ & $\mathrm{Na}$ & 2.9 & - & - & $0.3-1.5$ & 50 & - & Granular \\
\hline - $\mathbf{P}$ & • & $-\quad=$ & $-\mathrm{SO}_{3}^{-}$ & $\mathrm{Na}$ & 1.9 & - & - & $0.3-1.5$ & 50 & $\cdot$ & Granular \\
\hline$-c x$ & Weak & " & $-\infty^{-}$ & $\mathrm{Na}$ & 2.0 & - & - & $0.3-1.5$ & 30 & - & Granular \\
\hline$-\mathrm{CV}$ & $"$ & * & $-\mathrm{COO}^{-}$ & - & 4.0 & 1.0 & $40-50$ & $0.3-1.5$ & 40 & $0-8$ & Granuler \\
\hline Zerolit 215 & Strong & Zerolit Led., UK & $-\mathrm{SO}_{3}^{-}, \mathrm{OH}$ & $\mathbf{N a}$ & - & 0.92 & - & $0.3-1.2$ & 40 & $0-9$ & Granuler \\
\hline-210 & Weak & - & $-\mathrm{COO} . \mathrm{OH}$ & $\mathrm{H}$ & 2.5 & 1.1 & - & $0.3-1.2$ & 30 & $0-9$ & Granular \\
\hline
\end{tabular}




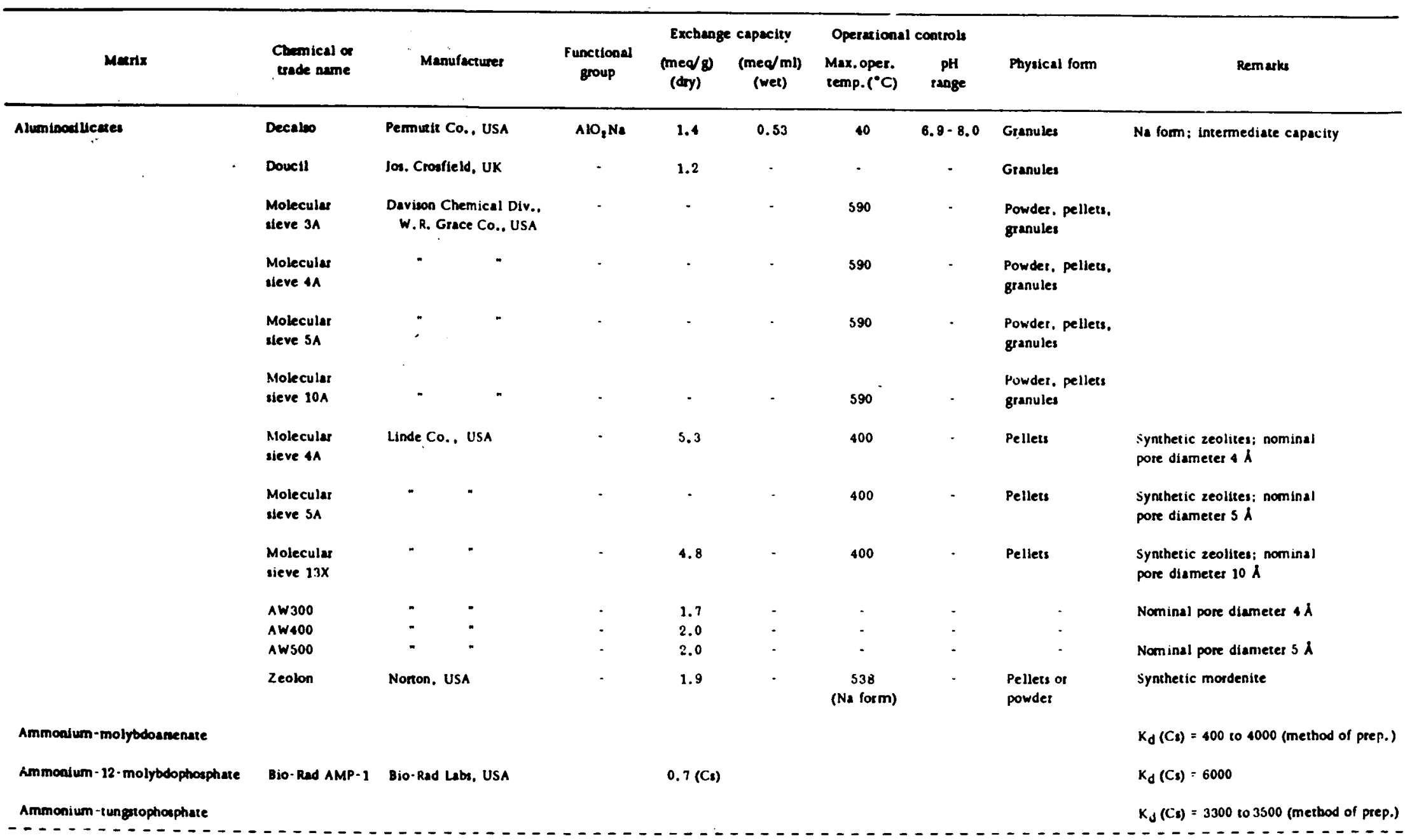


APPENDIX-II (Continued)

\begin{tabular}{|c|c|c|c|c|}
\hline Marix & Chemical of trade same & Menufectures & $\begin{array}{l}\text { Exchange cepacity } \\
\text { (med/g) (dy) }\end{array}$ & ments \\
\hline Arenares & $\begin{array}{l}\text { Ferric arsenate } \\
\text { Zircontum anserate }\end{array}$ & & $\begin{array}{l}\text { At pH 3.55-10.95: } 1.18-5.73(\mathrm{St}) \\
\text { At pH 3.12-10.68: } 2.87-6.15 \text { (SS) }\end{array}$ & \\
\hline Cerium aV compounds & $\begin{array}{l}\text { Ceric phoophaste, } \mathrm{H}^{+} \text {form: ceric } \\
\text { phopphate. } \mathrm{NH}_{4} \text { form: ceric oxide. } \\
\text { All nox available commercially }\end{array}$ & & At pH $0.10 \cdot 11.7: 0.07 \cdot 3.24$ & $\mathrm{Na}^{+}$loo-exchange capecity \\
\hline Ferrocyanide-molybdace & $\begin{array}{l}\text { Nor available commercially } \\
\text { FEM }\end{array}$ & & & \\
\hline Hydrows oxides & $\begin{array}{l}\text { Noe available commercially } \\
\mathrm{CO}(\mathrm{OH})_{3} \\
\mathrm{Sn}(\mathrm{OH})_{4} \\
\mathrm{Al}(\mathrm{OH}) \text {, } \\
\mathrm{Ni}(\mathrm{OH}), \\
\mathrm{Fe}(\mathrm{OH}), \\
\text { Lead sulphate (tribasic) }\end{array}$ & & $\begin{array}{l}\text { At pH 7-10:0.25-0.78 } \\
\text { At pH 4-11:0.13-1.78 } \\
\text { At pH 7-10:0.10-1.24 } \\
\text { At pH 6.5-10:0.02-2.25 } \\
\text { At pH 6-10:0.11:2.19 (St) } \\
\text { At pH 1-6.7:8.5-0.8 (anion exch.) } \\
\text { At pH 7.2-12.5:0.1-7.5 (cation exch) }\end{array}$ & $\begin{array}{l}\text { She paricles } \\
\text { While pariclea } \\
\text { Whiste particlea } \\
\text { Greed perticlea }\end{array}$ \\
\hline Phoophates & $\begin{array}{l}\text { ABEDEM Sn } \\
\text { ABEDEM TIA } \\
\text { ABEDEM TiB } \\
\text { ABEDEM } Z \mathbf{r}\end{array}$ & $\begin{array}{c}\text { SERAl, Belgium } \\
: \\
\vdots \\
:\end{array}$ & $\begin{array}{l}3.30(\mathrm{H}) \\
7.50(\mathrm{H}) \\
9.50 \mathrm{H} \\
5.20(\mathrm{H})\end{array}$ & \\
\hline Poxamium bexacyanocobelt (II) ferrate (III) & Not available commercially & & $6.0\left(C_{3}\right)$ & \\
\hline Quinoline-molybdophouphate & & & & $K_{d}\left(C_{1}\right)=1500 \mathrm{ml} / \mathrm{q}$ \\
\hline sillicures & $\begin{array}{l}\mathrm{Al}_{2} \mathrm{O}_{3} \cdot \times \mathrm{SiO}_{2} \\
\mathrm{SNO}_{2} \cdot \times \mathrm{SiO}_{2} \\
\mathrm{AnO} \cdot \times \mathrm{SiO}_{2} \\
(\mathrm{X}=\text { varying number of silicatcs })\end{array}$ & & & \\
\hline Thotium-bace & Thorium phusphate & & At pH 3.0-11.0:0.30-1.1 & \\
\hline Ttrenium-bese & Titanium phosphate & & At pH 2.5-11.5: $0.20-3.5$ & \\
\hline Ziroonium-base & 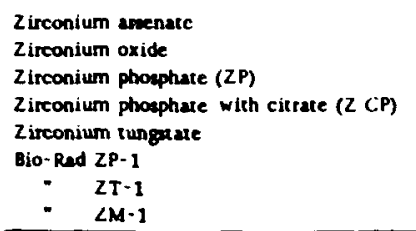 & Bio-Rad Labs, USA & $\begin{array}{l}\text { At pH } 2.5-1.30: 0.30-3.0 \\
\text { At pH } 3.5-11.5: 0.10-5.5 \\
\text { At pH } 2.5-12.0: 0.3-1.5 \\
1.0\end{array}$ & \\
\hline
\end{tabular}




\section{APPENDIX-II (Continued)}

\section{NATURAI, CATION EXCHANGERS}

a. Organic

\begin{tabular}{|c|c|c|c|c|c|c|c|c|c|}
\hline \multirow[b]{2}{*}{ Matrix } & \multirow[b]{2}{*}{$\begin{array}{l}\text { Mineral or } \\
\text { trade name }\end{array}$} & \multirow[b]{2}{*}{ Manufacturer } & \multirow[b]{2}{*}{$\begin{array}{l}\text { Functional } \\
\text { group }\end{array}$} & \multicolumn{2}{|c|}{ Exchange capacity } & \multicolumn{2}{|c|}{ Operational controls } & \multirow[b]{2}{*}{$\begin{array}{l}\text { Physical } \\
\text { form }\end{array}$} & \multirow[b]{2}{*}{ Remarks } \\
\hline & & & & $\begin{array}{c}(\mathrm{mea} / \mathrm{g}) \\
\text { (dry) }\end{array}$ & $\begin{array}{l}\text { (mea/ml) } \\
\text { (wet) }\end{array}$ & $\begin{array}{l}\text { Max. oper. } \\
\text { temp. }\left({ }^{\circ} \mathrm{C}\right)\end{array}$ & $\begin{array}{c}\mathrm{pH} \\
\text { range }\end{array}$ & & \\
\hline \multirow[t]{4}{*}{ Coals } & Dusarit S & DMACTI, Netherlands & $\begin{array}{l}-\mathrm{SO}_{3} \\
-\mathrm{COONa}\end{array}$ & & $\begin{array}{l}0.7 \text { (strong) } \\
0.35 \text { (weak) }\end{array}$ & 80 & $0-14$ & Granules & Polyfunctionsl \\
\hline & Imac C 19 & " & $\begin{array}{l}-\mathrm{SO}_{j}^{-} \\
-\mathrm{COOH}\end{array}$ & & $\begin{array}{l}0.3 \text { (strong) } \\
1.0 \text { (weak) }\end{array}$ & 80 & $4-14$ & Granules & Polyfunctional \\
\hline & Soucol & Jos. Crosfield, UK & -so; & 1.8 & 0.6 & 30 & & Granules & \\
\hline & Zeokarb-H & Permutit Co. , USA & $\begin{array}{l}-\mathrm{SO}_{3}^{-} \\
-\mathrm{COOH}\end{array}$ & 1.6 & 0.56 & 70 & 0.8 .5 & Granules & $\begin{array}{l}\text { Intermediate } \\
\text { acidity }\end{array}$ \\
\hline $\begin{array}{l}\text { Brown coal } \\
\text { (lignite) }\end{array}$ & $\begin{array}{l}\text { Local - Mol, Belgium } \\
\text { Idaho, USA }\end{array}$ & & $-\mathrm{COOH}$ & $\begin{array}{l}0.45 \\
\text { At pH } 4.1: 0.02-1.08\end{array}$ & & & & & \\
\hline Peat & Local - Poland & & & $0.05-0.4$ & & & & & \\
\hline
\end{tabular}

b. Inorganic

$\begin{aligned} & \text { Greensand } \\ & \text { (arbilized) }\end{aligned}$
$\begin{aligned} & \text { Greensand } \\ & \text { (atabilized) }\end{aligned}$
Zerolit


APPENDIX-II (Continued)

\begin{tabular}{|c|c|c|c|c|}
\hline Chy minerals & Kaolinite & $0.03-0.15$ & & \\
\hline \multirow{9}{*}{ (natural) } & Halloysite $2 \mathrm{H}_{2} \mathrm{O}$ & $0.05-0.10$ & & \\
\hline & Halloysite $4 \mathrm{H}_{2} \mathrm{O}$ & $0.40-0.50$ & & \\
\hline & Montmorillonitc & $0.80-1.5 c$ & & \\
\hline & Illite & $0.10-0.40$ & & \\
\hline & Vermiculite & $1.00-1.50$ & & \\
\hline & Chlorite & $0.10-0.40$ & & \\
\hline & Sepiolite-attapulgite & $0.20-0.30$ & & \\
\hline & Palygorskite & & & \\
\hline & Analcite & 4.50 & & \\
\hline \multirow{12}{*}{$\begin{array}{l}\text { Zeolite minerals } \\
\text { (natural) }\end{array}$} & Clinoptiloliti & 1.5 & $2.8 \cdot 3.2$ & Granules \\
\hline & & $1.61 \pm 0.01$ & & Granules \\
\hline & & 1.7 & & Granules \\
\hline & & At pH $4.1: 0.35$ & & Grenule: \\
\hline & Cancrinitu. & 10.0 & & \\
\hline & Erionite & 2.2 & & \\
\hline & Heulandite & 3.30 & & \\
\hline & Mordenite & 1.40 & & \\
\hline & Natrolite & 5.3 & & \\
\hline & Phillipsite & 2.3 & & \\
\hline & Sodalite & 9.2 & & \\
\hline & Ultramarine & 8.3 & & \\
\hline \multirow[t]{6}{*}{ Pyroclastic rocks } & Andesitic tuff & $0.10 \cdot 0.40$ & & \\
\hline & Rhyolitic tuff & $0.0-0.30$ & & \\
\hline & Rhyodacitic tuff & $0.40-1.30$ & & \\
\hline & Basaltic tuff & $0.30 \cdot 0.50$ & & \\
\hline & Tefritic \& fonolitic tuff & $0.20-0 . \times 0$ & & \\
\hline & Tuffites & $0.0 \cdot 0.50$ & & \\
\hline
\end{tabular}


APPENDIX-II (Continued)

SYNTHETIC ANION EXCHANGERS

a. Strong base polystyrene addition polymers Type I [ Rnetional group - $\mathrm{N}\left(\mathrm{CH}_{3}\right)_{3}$ ]

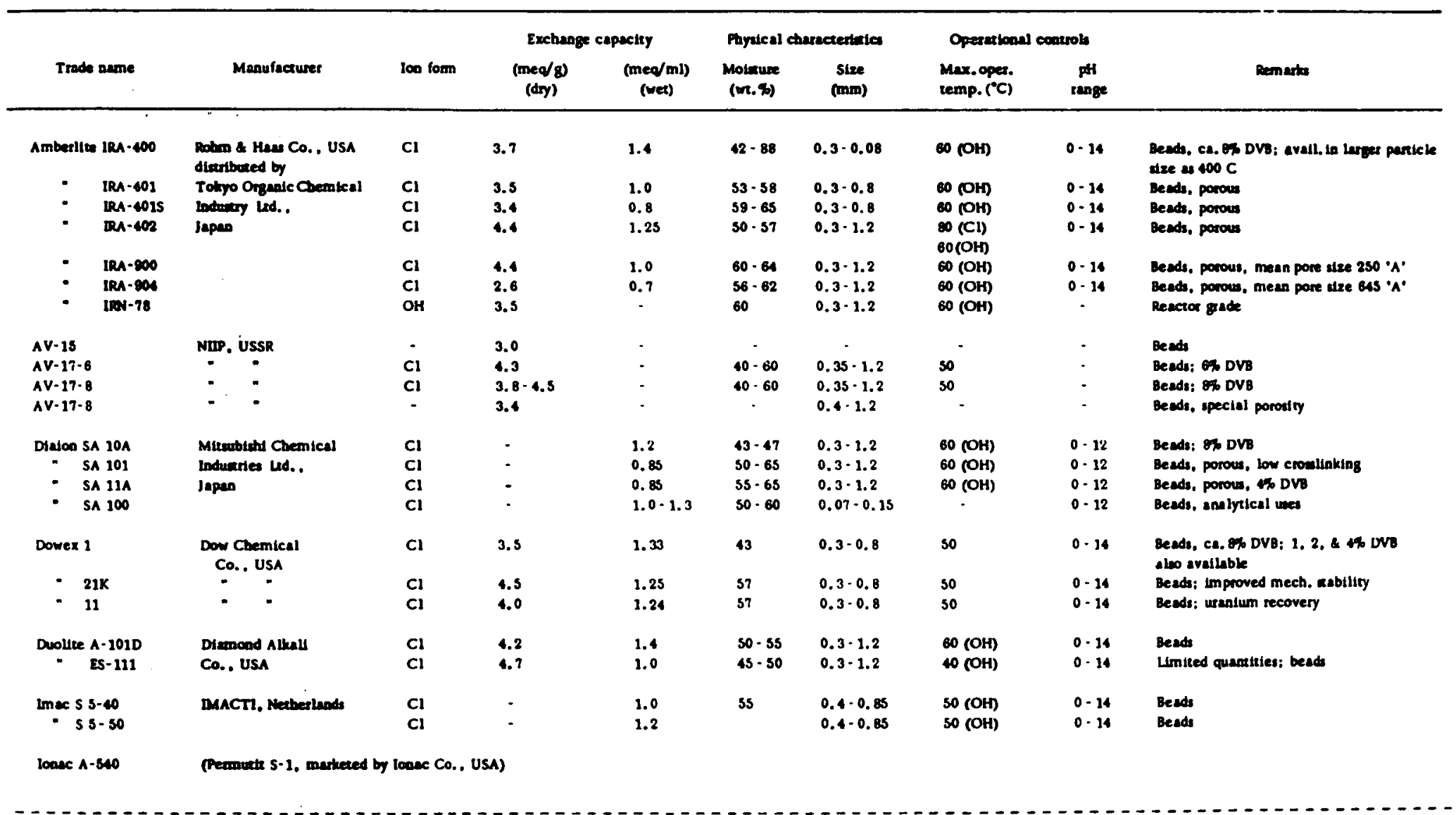


APPENDIX-II (Continued)

\begin{tabular}{|c|c|c|c|c|c|c|c|c|c|}
\hline $\begin{array}{r}\text { Kartel A-500 } \\
\text { A-soop }\end{array}$ & Montecatini, Ituly & $\begin{array}{l}\mathrm{Cl} \\
\mathrm{Cl}\end{array}$ & $\begin{array}{l}3.0 \\
3.3\end{array}$ & $\dot{-}$ & : & $\begin{array}{l}0.3 \cdot 1.0 \\
0.3 \cdot 1.0\end{array}$ & $\begin{array}{l}60 \text { (OH) } \\
60 \text { (OH) }\end{array}$ & $\begin{array}{l}0.14 \\
0-14\end{array}$ & 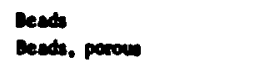 \\
\hline Lewatti M-500 & Fabeafabriken & $\mathrm{Cl}$ & 4.0 & 1.6 & - & $0.3 \cdot 1.2$ & 70 & 0.14 & nevede \\
\hline ISP 500 & Bayer A.G.. & $\mathrm{Cl}$ & 4.0 & 1.2 & - & $0.3-1.6$ & 70 & 0.14 & Cende, proven \\
\hline$=M-000$ & Fed. Rep. of Germany & $\cdot$ & 3.7 & 1.6 & - & $0.3 \cdot 1.2$ & \$0 & 0.14 & nende, porow \\
\hline$\because A \mid P \cdot 600$ & & - & 3.7 & 1.1 & - & $0.3-1.5$ & 40 & 0.14 & Deach, poroun \\
\hline Nalcite SBR & (Dowex 1. marketed by $\mathrm{Na}$ & o che & O.. USA) & & & & & & \\
\hline - SBR-P & & & & & & & & & Ponows \\
\hline Permutic ESB & Permurtt A.G., & $\mathrm{Cl}$ & 3.2 & 1.2 & - & - & 70 & - & Deents \\
\hline$=\quad$ ESB-26 & Fed. Rep. of Germany & $\mathrm{Cl}$ & $\mathbf{3 . 3}$ & 1.3 & - & - & 70 & - & Beeds \\
\hline Permutit S-I & Permutit Co.. & $\mathrm{Cl}$ & 3.6 & 1.0 & $50-60$ & $0.3 \cdot 1.2$ & 100 & 0.14 & Besds \\
\hline - Nsi & USA & $\mathrm{OH}$ & 3.5 & 1.0 & $50 \cdot 60$ & $0.3 \cdot 1.2$ & $\infty$ & 0.14 & Beads, nuclear grade \\
\hline Resenex HBL & los. Crosfield. & - & 3.5 & 1.5 & . & - & 60 & - & Beach \\
\hline$=\mathrm{HBT}$ & UK & - & 3.5 & & - & $\cdot$ & 60 & - & Beads \\
\hline$S-E-T A$ anex & $\begin{array}{l}\text { Výzkumný ústav } \\
\text { symbineticikých } \\
\text { pryakyñc a lakt, } \\
\text { Pardubice. CSSR }\end{array}$ & $\mathrm{Cl}$ & 3.45 & 1.35 & - & $0.3 \cdot 0.8$ & $60(\mathrm{OH})$ & 0.12 & \\
\hline Wolfatit ES & VEB Farbenfabrik & $\mathrm{Cl}$ & 3.5 & 0.5 & $65-75$ & $0.5-2.0$ & 60 (OH) & $2 \cdot 10$ & \\
\hline " SBT & Wolfen, & $\mathrm{Cl}$ & 3.0 & 0.9 & $53-63$ & $0.6 \cdot 2.0$ & & 0.14 & Uranium recovery, beads \\
\hline $\begin{array}{ll}n & \text { SBW } \\
& \text { RO }\end{array}$ & German Democtatic Rep. & $\begin{array}{l}\mathrm{Cl} \\
\mathrm{OH}\end{array}$ & 3.5 & $\begin{array}{l}0.9 \\
0.9\end{array}$ & $58 \cdot 68$ & $\begin{array}{l}0.3 \cdot 1.5 \\
0.3-1.5\end{array}$ & $\begin{array}{l}60 \\
60\end{array}$ & $\begin{array}{l}0.11 \\
0.14\end{array}$ & Beads \\
\hline $\begin{array}{r}\text { Zerolit FF-1P } \\
K-M P\end{array}$ & Zerolit ud., & $\mathrm{Cl}$ & 4.0 & 1.2 & - & $0.3-1.2$ & 60 & : & Leads, 7 - $0 \%$ DV \\
\hline \multicolumn{10}{|c|}{$\begin{array}{l}\text { b. Strong base polystyrene addition polymers } \\
\text { Type II [functional group - } \mathrm{N}\left(\mathrm{CH}_{2} \mathrm{OH}\right)\left(\mathrm{CH}_{3}\right) \text { I }\end{array}$} \\
\hline imberlite IRA-410 & Rothm \& Has Co., USA & $\mathrm{Cl}$ & 3.3 & 1.40 & $40-45$ & $0.3 \cdot 0.8$ & 40 (OH) & $0-14$ & Approx. Ox DVB, beeds \\
\hline - IRA-910 & dieributed by & $\mathrm{Cl}$ & - & 1.10 & $55 \cdot 60$ & $0.3 \cdot 0.8$ & $40(\mathrm{OH})$ & 0.14 & Deads; macroseticulas \\
\hline - $\quad \mathbb{R A}-911$ & $\begin{array}{l}\text { Tokyo Organic Chem. } \\
\text { lnd. Led., Japan }\end{array}$ & $\mathrm{cl}$ & 2.7 & 0.9 & $42-44$ & $0.3 \cdot 1.2$ & $40(\mathrm{OH})$ & $0-14$ & Meeds; mecrorealcules \\
\hline Diajoo SA 20A & Mireubiubi Cbemical & $\mathrm{Cl}$ & - & 1.3 & $40-45$ & $0.3 \cdot 1.2$ & 40 (OH) & $0-12$ & reads \\
\hline - SA 21A & Ind. Ld., Japan & $\mathrm{cl}$ & - & 0.8 & $55-65$ & $0.3 \cdot 1.2$ & 10 (OH) & 0.12 & Deade, porower \\
\hline
\end{tabular}


1

APPENDIX-II (Contınued)

\begin{tabular}{|c|c|c|c|c|c|c|c|c|c|}
\hline \multirow[b]{2}{*}{ Trade aame } & \multirow[b]{2}{*}{ Manufacturet } & \multirow[b]{2}{*}{ Ion form } & \multicolumn{2}{|c|}{ Exchange capactiy } & \multicolumn{2}{|c|}{ Phyzical characteristics } & \multicolumn{2}{|c|}{ Opezational controls } & \multirow[b]{2}{*}{ Remarks } \\
\hline & & & $\begin{array}{l}(\mathrm{med} / \mathrm{g}) \\
\text { (dry) }\end{array}$ & $\begin{array}{c}\text { (med/ml) } \\
\text { (wet) }\end{array}$ & $\begin{array}{l}\text { Molsture } \\
\text { (wt.\%) }\end{array}$ & $\begin{array}{l}\text { Size } \\
(\mathrm{mm})\end{array}$ & $\begin{array}{l}\text { Max.oper. } \\
\text { temp. }(" O)\end{array}$ & $\begin{array}{l}\text { pH } \\
\text { range }\end{array}$ & \\
\hline Dowex 2 & Dow Chemical Co.. USA & $\mathrm{Cl}$ & 3.5 & 1.33 & 37 & $0.3-0.8$ & $30(\mathrm{OH})$ & $0-14$ & Beads, ca. $8 \%$ DVB; $4 \%$ DVB atso available \\
\hline Duolite A-102D & $\begin{array}{l}\text { Diamond Alkali } \\
\text { Co., USA }\end{array}$ & $\mathrm{Cl}$ & 4.2 & 1.4 & $45-50$ & $0.3-1.2$ & $40(\mathrm{OH})$ & $0 \cdot 14$ & Beads, porous \\
\hline Imac 55-52 & IMACTI, Netherlands & $a$ & & 1.2 & - & $0.4-0.85$ & $40(\mathrm{OH})$ & $0 \cdot 14$ & Beads \\
\hline Ionac A-550 & \multicolumn{6}{|c|}{ (Permutit S-2, marketcd by lunac Co.. USA) } & & & \\
\hline Kascel A-300 & Montecatini, Italy & (:) & 3.2 & $\cdot$ & 45 & $0.3 \cdot 1.2$ & $40(\mathrm{OH})$ & $0 \cdot 16$ & Beade \\
\hline Nalcite SAR & \multicolumn{6}{|c|}{ (Dowex 2, marketed by Nalco (hemical Co.. USA) } & & & \\
\hline Permutit ES & Permutit A.G.. & Cl & 3.2 & 1.2 & $\cdot$ & $\cdot$ & 40 & $\cdot$ & Beads \\
\hline - ES-26 & Fed. Rep. of Germany & $\mathrm{Cl}$ & 3.3 & 1.3 & $\cdot$ & - & 40 & $\cdot$ & Beads \\
\hline Permutit S-2 & Permurit Co., USA & $\begin{array}{l}\mathrm{Cl} \text {. } \\
\mathrm{OH} \text { : } \mathrm{SO}_{3}\end{array}$ & $\begin{array}{l}3.5(\mathrm{Cl}) \\
3.5\left(\mathrm{OH}, \mathrm{SO}_{3}\right)\end{array}$ & 1.3 & $45-.55$ & $0.3 \cdot 1.2$ & $\begin{array}{l}100(\mathrm{Cl}) \\
60\left(\mathrm{OH}, \mathrm{SO}_{3}\right)\end{array}$ & $0-14$ & Beads \\
\hline S-8-D anex & $\begin{array}{l}\text { Výzkumný ústav } \\
\text { synthetických } \\
\text { pryskyñic a laka, } \\
\text { Pardubice. CSSR }\end{array}$ & $\mathrm{Cl}$ & 3.45 & 1.35 & $\cdot$ & $0.3-0.8$ & $40(\mathrm{OH})$ & $0 \cdot 12$ & Beads \\
\hline Wolfatit SBK & $\begin{array}{l}\text { VEB Farbenfabrik } \\
\text { Wolfen, German } \\
\text { Democratic Republic }\end{array}$ & $\mathrm{Cl}$ & 3.0 & 1.0 & $35-45$ & $0.3 \cdot 1.5$ & $40(\mathrm{OH})$ & $0-10.5$ & Beads, 6-8\% DVB \\
\hline Zerolit N-1P & Zerolit Ld.. & $\mathrm{Cl}$ & - & 1.10 & $\cdot$ & $0.3 \cdot 1.2$ & 40 & $\begin{array}{l}0-14 \\
0-14\end{array}$ & $\begin{array}{l}\text { Beads: isoporous } \\
\text { Beadi; isoporous }\end{array}$ \\
\hline - P-1P & UK & $\mathrm{Cl}$ & - & 1.14 & - & $0.3-1.2$ & 40 & $0-14$ & Beads; 1soporous \\
\hline
\end{tabular}


APPENDIX-II (Continued)

c. Mledium and weak base polystyrene addition polymers

\begin{tabular}{|c|c|c|c|c|c|c|c|c|c|c|}
\hline \multirow[b]{2}{*}{ Trade neme } & \multirow[b]{2}{*}{ Menulacturer } & \multirow[b]{2}{*}{$\begin{array}{l}\text { Functional } \\
\text { group }\end{array}$} & \multirow[b]{2}{*}{ loo form } & \multicolumn{2}{|c|}{ Exchange capacity } & \multicolumn{2}{|c|}{ Phyrical charecterivetea } & \multicolumn{2}{|c|}{ Operational conctob } & \multirow[b]{2}{*}{ Menarta } \\
\hline & & & & $\begin{array}{c}(\operatorname{mes} / g) \\
(\mathbb{d r y})\end{array}$ & $\begin{array}{l}\text { (mea/ml) } \\
\text { (wea) }\end{array}$ & $\begin{array}{l}\text { Moleture } \\
(m . x)\end{array}$ & $\begin{array}{c}\text { Sise } \\
(\mathrm{mm})\end{array}$ & $\begin{array}{l}\text { Max. oper. } \\
\text { temp. }\left({ }^{\circ} \mathrm{C}\right)\end{array}$ & $\underset{\text { natere }}{\text { PH }}$ & \\
\hline 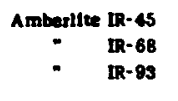 & Botm \& Heas Co., USA & $\begin{array}{l}\text { Weak base } \\
\text {-N(R), } \\
-\end{array}$ & $\begin{array}{c}\text { Freebase } \\
:\end{array}$ & $\begin{array}{l}5.0 \\
6 \\
4.8\end{array}$ & $\begin{array}{l}1.9 \\
1.60 \\
1.4\end{array}$ & $\begin{array}{l}10-45 \\
57-63 \\
46-54\end{array}$ & $\begin{array}{l}0.3-0.84 \\
0.3-1.2 \\
0.3-1.2\end{array}$ & $\begin{array}{l}100(\mathrm{OH}) \\
60 \\
100(\mathrm{OH})\end{array}$ & $\begin{array}{l}0-9 \\
0-9 \\
0-\theta\end{array}$ & 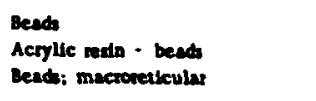 \\
\hline$A N-18-6$ & MIP, USSR & Amino groupe & $\mathrm{Cl}$ & 3.5 & - & $40-60$ & $0.35-1.2$ & - & - & Dead \\
\hline AN-23 & $=\dot{ }$ & & Cl & 5.0 & - & 30 & $0.25-1.0$ & 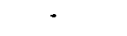 & - & Beach \\
\hline AN-25 & $: \quad:$ & $-\cdot-$ & $\mathrm{Cl}$ & 5.0 & - & 30 & $0.25 \cdot 1.0$ & - & - & Beand \\
\hline AV-20 & $: \quad:$ & Pyridine base & $\mathrm{Cl}$ & 3.5 & - & so & $0.25-1.0$ & 130 & - & Denás \\
\hline Av-23 & $\cdot$ & $\because \quad=$ & $\mathrm{Cl}$ & 3.5 & - & 60 & $0.25-1.0$ & 130 & $\cdot$ & Beach \\
\hline Dowex 3 & $\begin{array}{l}\text { Dow Chemical } \\
\text { Co.. USA }\end{array}$ & $\begin{array}{l}\text { Weak base } \\
\text { amino groupe }\end{array}$ & $\mathbf{O H}$ & 5.5 & 2.5 & 35 & $0.3 \cdot 0.84$ & 65 & - & seacts \\
\hline Duolie A-14 & $\begin{array}{l}\text { Diamood Alkall } \\
\text { Co.. USA }\end{array}$ & $\begin{array}{l}\text { Weak base } \\
\text { amino groups }\end{array}$ & OH & 8.0 & 2.5 & - & $0.3 \cdot 1.2$ & - & - & Beads \\
\hline $\operatorname{Im} \propto \mathrm{A} 20$ & $\begin{array}{l}\text { IMACT. } \\
\text { Netheriands }\end{array}$ & $\begin{array}{l}\text { Weak baxe } \\
\text { amino groups }\end{array}$ & OH & - & - & - & $0.4-0.85$ & 100 & $0-8$ & $\begin{array}{l}\text { Beads; prim., sec. \& } \\
\text { tertiary aminose }\end{array}$ \\
\hline$-A 21$ & & $=$ & он & $\cdot$ & $\cdot$ & $\cdot$ & $0.4 \cdot 0.85$ & 100 & 0.8 & Beads; tertiery amino groupe only \\
\hline loase A-315 & (Permurit $W$, marketed $b$ & Ionac Co., USA) & & & & & & & & \\
\hline Lewatit MP- 60 & $\begin{array}{l}\text { Faubenfabriken } \\
\text { Bayer A.G., } \\
\text { Fed. Rep.of Germany }\end{array}$ & $\begin{array}{l}\text { Weak base } \\
\text { amino groups }\end{array}$ & - & 0.3 & 2.2 & $40-50$ & $0.3 \cdot 1.5$ & 100 & 0.14 & Beads; mseroporous \\
\hline Nakite WOR & (Dowex 3, marketed by ? & alco Chemical Co. & . USA) & & & & & & & \\
\hline Permurit $w$ & $\begin{array}{l}\text { Permutit Co.. } \\
\text { USA }\end{array}$ & $\begin{array}{l}\text { Weak base } \\
\text { amino groups }\end{array}$ & $\mathrm{Cl}$ & 4.5 & 1.3 & $45 \cdot 551$ & $0.3 \cdot 2.0$ & 95 & - & Beads \\
\hline Wolfatiz Y13 & $\begin{array}{l}\text { VEB Farbenfabrik } \\
\text { Wolfen. German } \\
\text { Democraric Rep. }\end{array}$ & $\begin{array}{l}\text { Weak base } \\
\text { amino groups }\end{array}$ & OH & . & 1.25 & $\cdot$ & $0.3-1.2$ & - & $0-14$ & Beacte \\
\hline Zerolit G & Zerolit Led., UK & Amino groups & $\mathrm{Cl}$ & 3.5 & 1.6 & - & $0.3-1.2$ & 100 & - & $\left.-\mathrm{N}_{4} \mathrm{C}_{2} \mathrm{H}_{3}\right)_{\mathrm{s}}$ groups ooly: beads \\
\hline $\bar{M}$ & $: \quad:$ & & $\mathrm{Cl}$ & $\begin{array}{l}5.5 \\
3.8\end{array}$ & $\begin{array}{l}1.9 \\
1.28\end{array}$ & $\dot{-}$ & $\begin{array}{l}0.3 \cdot 1.2 \\
0.3 \cdot 1.2\end{array}$ & $\begin{array}{l}60 \\
70\end{array}$ & $\therefore$ & Beeds \\
\hline - $\mathbf{H}$ & & $\begin{array}{l}\text { Weak \& srong } \\
\text { base groups }\end{array}$ & & 3.8 & & $\cdot$ & $0.3 \cdot 1.2$ & 70 & & \\
\hline
\end{tabular}




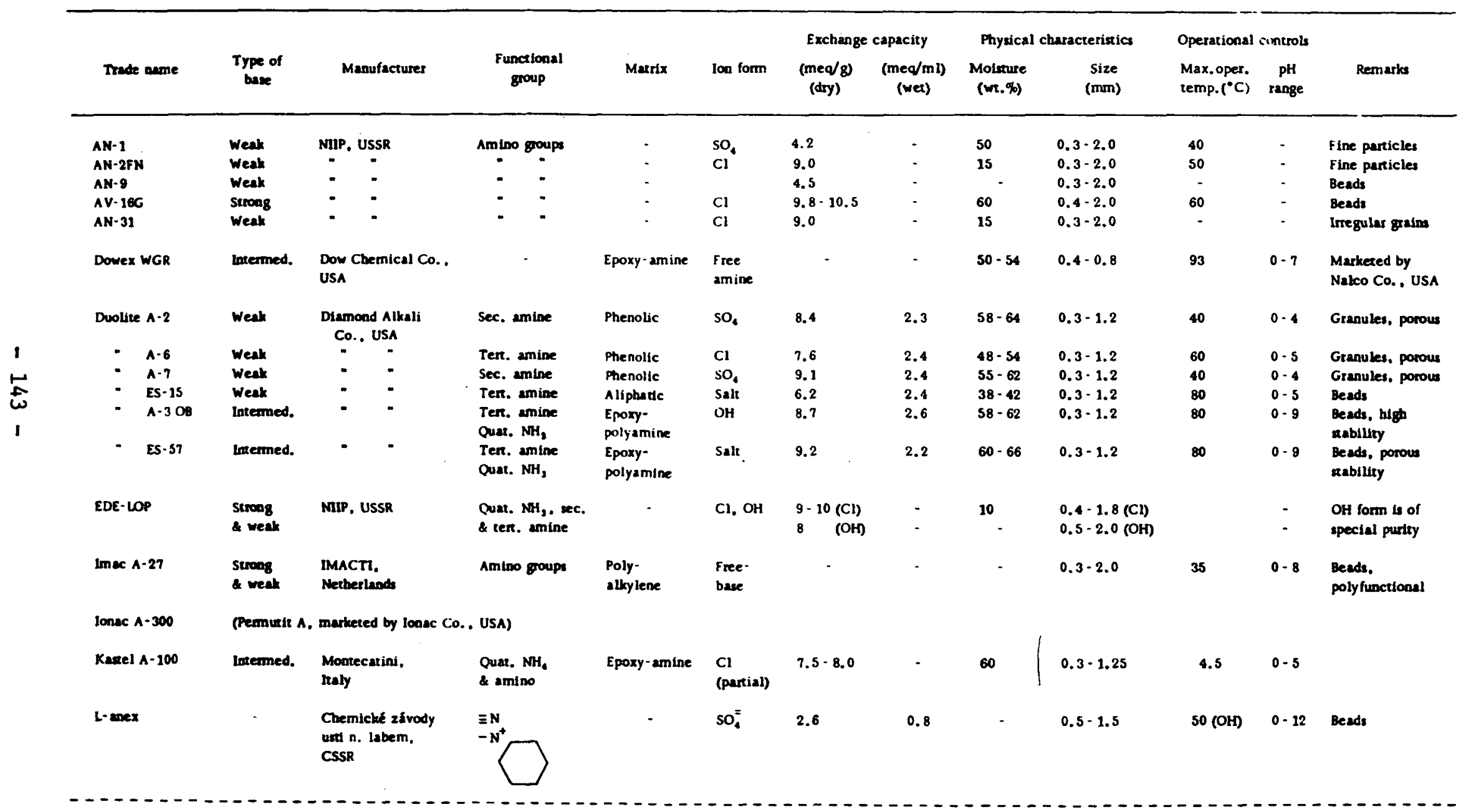


APPENDIX-II (Continued)

\begin{tabular}{|c|c|c|c|c|c|c|c|c|c|c|c|c|}
\hline $\begin{array}{l}\text { Lewath yaH } 59 \\
-\quad \text { MN }\end{array}$ & $\begin{array}{l}\text { Weak } \\
\text { streag }\end{array}$ & $\begin{array}{l}\text { Farbenfabriken } \\
\text { Bayer A.G.. } \\
\text { Fed. Rep. of Germ any }\end{array}$ & $\begin{array}{l}\text { Amino } \\
-N(a l k y l) ;\end{array}$ & $\dot{-}$ & $\dot{-}$ & $\begin{array}{l}6.0 \\
2.3\end{array}$ & $\begin{array}{l}2.4 \\
0.9\end{array}$ & $\begin{array}{l}40-50 \\
46-54\end{array}$ & $\begin{array}{l}0.3-1.2 \\
0.3-2.0\end{array}$ & $\begin{array}{l}30 \\
30\end{array}$ & $\begin{array}{l}0.14 \\
0.14\end{array}$ & Gramilar \\
\hline Lewadi AIN & Weak & $\begin{array}{l}\text { Moskovak Chem. } \\
\text { Technol. Inst. USSR }\end{array}$ & $=\mathrm{NH}, \equiv \mathrm{N}$ & - & - & 4.1 & $\cdot$ & - & $0.3-2.0$ & - & - & Flae particles \\
\hline Mtyltion G & Weak & MUKY, Hungary & $\equiv \mathbf{N}$ & $\cdot$ & - & 4.6 & 1.25 & - & - & - & $\cdot$ & $\begin{array}{l}\text { Gumaldive. } \\
\text { tocmaldebyde }\end{array}$ \\
\hline $\begin{array}{l}\text { N } \\
\text { NO }\end{array}$ & $\begin{array}{l}\text { Weak } \\
\text { Weak }\end{array}$ & $\begin{array}{l}\text { Moskovak Chem. } \\
\text { Technol. Inst. . USSR }\end{array}$ & $\begin{array}{l}\equiv N_{1} \equiv N_{0} \\
=N H_{0} \equiv N_{0}-N R_{3}\end{array}$ & $\dot{-}$ & $\dot{-}$ & $\begin{array}{l}4.1 \\
4.1\end{array}$ & $\dot{r}$ & - & $\begin{array}{l}0.3-2.0 \\
0.3-2.5\end{array}$ & $\dot{-}$ & $\dot{-}$ & $\begin{array}{l}\text { Fine particles } \\
\text { Flee parieles }\end{array}$ \\
\hline PEK & $\begin{array}{l}\text { Strong } \\
\text { \& weak }\end{array}$ & $\begin{array}{l}\text { Gosud. Inattut } \\
\text { Prtk1.K. . USSR }\end{array}$ & $=\mathrm{NH}_{2}, \equiv \mathrm{N}$ & - & $\cdot$ & 6.0 & - & $\cdot$ & $0.3-1.0$ & - & - & Fine paricles \\
\hline Permudt E-3 & Weak & $\begin{array}{l}\text { Permutit A.G.. } \\
\text { Fed. Rep. of }\end{array}$ & Amino groups & - & - & 6.0 & - & $\cdot$ & $0.3-1.0$ & - & $\cdot$ & $\begin{array}{l}\text { Grancles, }-\mathrm{Mn} \text {, } \\
\text { groupe oaly }\end{array}$ \\
\hline - $E-7 P$ & Wesk & Germany & $\cdot \quad-$ & - & $\cdot$ & 6.0 & - & - & - & 40 & - & $\begin{array}{l}\text { Gresules. Wirity } \\
\text { porous }\end{array}$ \\
\hline Permutit $A$ & Intermed. & Permutit Co., USA & $N R_{3}, N_{1}$ & $\begin{array}{l}\text { Aliphatic } \\
\text { polyamine }\end{array}$ & $\mathrm{Cl}-\mathrm{sa}$ & 5.5 & 1.8 & $1-5$ & $0.3-1.2$ & 40 & $0-12$ & Granules \\
\hline$\therefore \quad A B$ & $"$ & 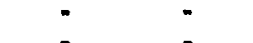 & $N R_{3}, N_{4}$ & 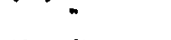 & $\mathrm{Cl} \cdot \mathrm{SO}_{4}$ & 5.5 & 1.8 & $1-5$ & $0.3-1.2$ & 40 & $0-12$ & Beads \\
\hline$\cdot \quad$ CCG & • & - & $N R_{3}$ & Phenolic & $\mathrm{Cl}-\mathrm{SO}_{4}$ & 5.5 & 1.6 & $2-7$ & $0.3-1.2$ & 60 & $0-12$ & Granules \\
\hline - Deacidite & weak & - & $\mathrm{NHR}_{2}, \mathrm{NR}$, & $\begin{array}{l}\text { Aliphatic } \\
\text { polyamine }\end{array}$ & $\mathrm{Cl}-\mathrm{SO}_{4}$ & 5.5 & 1.8 & $1-5$ & $0.3 \cdot 1.2$ & 40 & $0-12$ & Granules \\
\hline Resanex & Weak & los. Crosfield, UK & Amino groups & $\cdot$ & - & 9.0 & 3.0 & - & - & 80 & 0.9 & Grenules \\
\hline Wolfatit $x$ & Weak & $\begin{array}{l}\text { VEB Fabenfabrik } \\
\text { Wolfen, German } \\
\text { Democratic Rep. }\end{array}$ & Amino groups & - & $\mathrm{Cl}$ & 4.3 & $\cdot$ & $\cdot$ & $0.3-1.5$ & 30 & $\cdot$ & Granules \\
\hline Zerolit E & Medium & Zerolit LAd., UK & Amino groups & $\begin{array}{l}\text { Phenol } \\
\text { formaldehyde }\end{array}$ & $\begin{array}{l}\text { Free- } \\
\text { base }\end{array}$ & & 1.6 & - & $0.3-1.2$ & 30 & $0-12$ & Granules \\
\hline
\end{tabular}


APPENDIX-II (Continued)

MIXED BED ION-EXCHANGE RESINS

\begin{tabular}{|c|c|c|c|c|c|}
\hline Trade name & Manufacturer & $\begin{array}{c}\text { Cation } \\
\text { exchange resin }\end{array}$ & $\begin{array}{c}\text { Anion } \\
\text { exchange resin }\end{array}$ & $\begin{array}{l}\text { Approx. vol. ratios } \\
\text { cation : anion }\end{array}$ & Remarks \\
\hline Amberlite MB-1 & Rohm \& Haas Co. . USA & IR-120 & IRA 400 or 401 & $1: 1.5$ & \\
\hline$" \quad M B-2$ & & IR-120 & IRA 410 & $1: 1.5$ & \\
\hline MB-3 & & IR-120 & IRA 410 & $1: 1.5$ & Exhaustion colour indicator \\
\hline n $\quad \mathrm{MB}-4$ & & IR-120 & IR-45 & $1: 1$ & \\
\hline$\cdots \quad$ MB-5 & & IRC -50 & IRA 410 & $1: 1.5$ & \\
\hline MB-6 & & IRC -50 & IR- 45 & - & Nuclear grade \\
\hline IRN-217 & & IRN-218( $\left.{ }^{7} \mathrm{Li}\right)^{+}$ & IRN-78 & - & Nuclear grade \\
\hline IRN-150 & & IRN- $77\left(\mathrm{H}^{+}\right)$ & IRN-78 & - & Nuclear grade \\
\hline$=\quad$ IRN-154 & & IRN-163 $\left(\mathrm{Li}^{+}\right)$ & IRN-78 & - & Nuclear grade \\
\hline$=\quad I R N-170$ & & IRN-169 $\left(\mathrm{NH}_{4}^{+}\right)$ & IRN-78 & - & Nuclear grade \\
\hline Bio-Deminrolit & Zerolit Ld., UK & Zerolit 225 & Zerolit FF-1P & - & Also available with indicator \\
\hline Nalcite MR-1 & Dow Chemical Co. . USA & HCR-W & SBR & $1: 1$ & Technical grade; indicator \\
\hline - MR-2 & distributed by & HCR & SRR & $1: 1$ & Nuclear grade \\
\hline " $M R-3$ & Nalco Chemical Co. . USA & HCR-W & SBR & $1: 1$ & Nuclear grade \\
\hline - $M R-5$ & & HCR-W $\left(K^{+}\right)$ & SBR & $1: 1$ & Nuclear grade \\
\hline - $\quad M R-6$ & & HCR-W $\left(\mathrm{Li}^{+}\right)$ & SBR & $1: 1$ & Nuclear grade \\
\hline " MR-7 & & HCR-W $\left(\mathrm{NH}_{4}^{+}\right)$ & SBR & $1: 1$ & Nuclear grade \\
\hline Wolfatit MBW & & KPS & SBW & $1: 2$ & \\
\hline$* \quad \mathbf{R O H}$ & & $\mathbf{R H}$ & RO & $1: 1$ & Nuclear grade \\
\hline * ROL & & RK & RO & $1: 1$ & Nuclear grade (LOH) \\
\hline - $\quad$ ROK & & RK & RO & $1: 1$ & Nuclear grade (KOH) \\
\hline$\cdots \quad$ RON & & RN & ROO & $1: 1$ & Nuclear grade $\left(\mathrm{NH}_{4} \mathrm{OH}\right)$ \\
\hline
\end{tabular}


APPENDIX-II (Continued)

ION-EXCHANGE MEMBRANES

a. Cation exchangers

\begin{tabular}{|c|c|c|c|c|c|c|c|c|c|}
\hline Track name & Mecoufectures & meatix & Funcertonal & $\begin{array}{l}\text { Exchenge } \\
\text { capectry } \\
\text { (med/o dy } \\
\text { membeane }\end{array}$ & 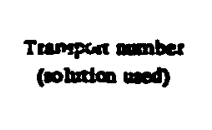 & 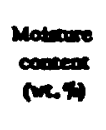 & Thenes & 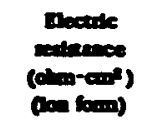 & menesis \\
\hline 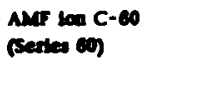 & $\begin{array}{l}\text { Amerteson Mectibe } \\
\text { \& Poundry. USA }\end{array}$ & $\begin{array}{l}\text { Polyectyleace- } \\
\text { ayseace } \\
\text { copolymen }\end{array}$ & $-\mathrm{so}_{3}$ & $1.5 \div 0.3$ & $\begin{array}{l}0.22 \pm 0.03 \\
(0.2 \Psi 0.1 \mathrm{~N} \text { KCD }\end{array}$ & 35 & $0.28-0.20$ & $5 \pm 2(x)$ & $\begin{array}{ll}91.5 \\
\text { wathe woll }\end{array}$ \\
\hline $\begin{array}{l}\text { ANT loo C-100 } \\
\text { (Sentea } 100)\end{array}$ & $\cdot$ & - & $-\mathrm{so}_{\mathbf{2}}^{-}$ & $1.3 \pm 0.3$ & $\begin{array}{l}0.28 \pm 0.1 \\
0.2 Y / 0.1 \mathrm{KCH}\end{array}$ & 25 & 0.20 & $7 \pm 2(10)$ & Boll \\
\hline ANA 2000 C-320 & - & $\begin{array}{l}\text { Polymeric } \\
\text { Propocation }\end{array}$ & -50 & $0.0 \pm 0.2$ & $\begin{array}{l}0.88 \pm 0.5 \\
0.5 \mathrm{y} / .0 \mathrm{~N} \mathrm{KCn}\end{array}$ & - & 0.28 & $4.6 \pm 2$ & moll \\
\hline $\begin{array}{l}\text { AMF } \ln C-913 \\
\text { (seried soos) }\end{array}$ & - & $\bullet$ & $-\infty$ & $0.8=0.2$ & $\begin{array}{l}0.20 \leq 0.003 \\
0.2 \mathbb{N} 0.1 \mathrm{KCl}\end{array}$ & 12 & 0.17 & $4.5=29$ & $112 \mathrm{cment}$ \\
\hline CK-1 & 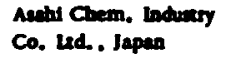 & Polynyrease & $-\mathbf{s o}_{3}$ & 2.6 & $\begin{array}{l}0.98 \\
(0.5 \mathrm{~N} N \mathrm{KCl})\end{array}$ & $\mathbf{3 6}$ & 0.23 & 4.2 & \\
\hline$M C-3112$ & $\begin{array}{l}\text { Penmuded Co., USA, } \\
\text { derestoced by }\end{array}$ & - & - & 1.06 & $\begin{array}{l}0.911 \\
0.5 \pm 1 \mathrm{~N} N \mathrm{NACD}\end{array}$ & - & 0.15 & $\begin{array}{l}3.4 \\
\text { (1.0N MuCp }\end{array}$ & Heverogenoour: \\
\hline$M C-3236$ & $\begin{array}{l}\text { lonece Chemieal Co.. } \\
\text { USA }\end{array}$ & - & - & 1.26 & $\begin{array}{l}0.853 \\
0.5 \mathrm{~N} / 1 \mathrm{~N} \text { NaCD }\end{array}$ & - & 0.30 & (1. $11 \mathrm{MaCl}$ & 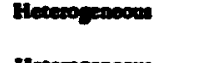 \\
\hline$M C-3470 X L$ & & - & - & 1.05 & $\begin{array}{l}0.962 \\
(0.5 \mathrm{~N} / 1 \mathrm{~N} \mathrm{MCD})\end{array}$ & - & 0.50 & (1.8) & Heveropeposons \\
\hline$M K-40 N$ & MmP, usss & $\begin{array}{l}\text { Polynyrease } \\
\text { (KU-2) }\end{array}$ & $-\mathrm{SO}^{-}$ & 2.3 & $\begin{array}{l}0.03 \\
(0.01 \mathrm{y} / 0.2 \mathrm{~N} \mathrm{NCD}\end{array}$ & 41 & $0.7-0.8$ & 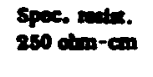 & 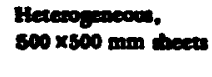 \\
\hline Nal fils 1 & Naloo Crem. Co., USA & - & $\cdot \mathbf{s o}^{\prime}$ & - & 0.81 & 20 & 0.09 & $\begin{array}{l}10-15(\infty) \\
0.154-1 \times C D\end{array}$ & $96.5 \mathrm{~cm}$ wide \\
\hline Stepeos CR-61 & booten be., USA & Polyzyrese & -so; & 2.8 & $0.9-0.85$ & $45 \cdot 50$ & 0.58 & $\begin{array}{l}5.3 \mathrm{Om}) \\
\text { (0.8n NuCD }\end{array}$ & Proven: \\
\hline senten c-100 & $\begin{array}{l}\text { lape oryes Co. } \\
\text { led. supen }\end{array}$ & - & -50 & - & $\begin{array}{l}0.9 \\
0.8 \mathrm{~N} / 2.5 \mathrm{~N} \text { MaCD }\end{array}$ & 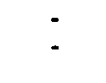 & $0.20-0.22$ & $\begin{array}{l}4.5 \\
0.5 \mathrm{NuCl}\end{array}$ & $\infty 0 \mathrm{~cm}$ wide sol] \\
\hline Selemtion CMC-10 & Anet Gime Co. & - & -50 & 1.08 & 0.91 & - & $0.20-0.25$ & 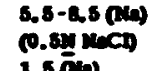 & $\operatorname{secmencm~}$ \\
\hline $\begin{array}{r}\text { CNG.80 } \\
-\end{array}$ & & $\cdot$ & $-\mathrm{SO}_{2}^{-}$ & - & $\begin{array}{l}0 . \infty \\
(0.5 \mathrm{~N} / 1.0 \mathrm{MCl})\end{array}$ & - & $0.28-0.28$ & 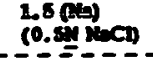 & 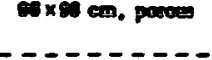 \\
\hline
\end{tabular}


APPENDIX-II (Continued)

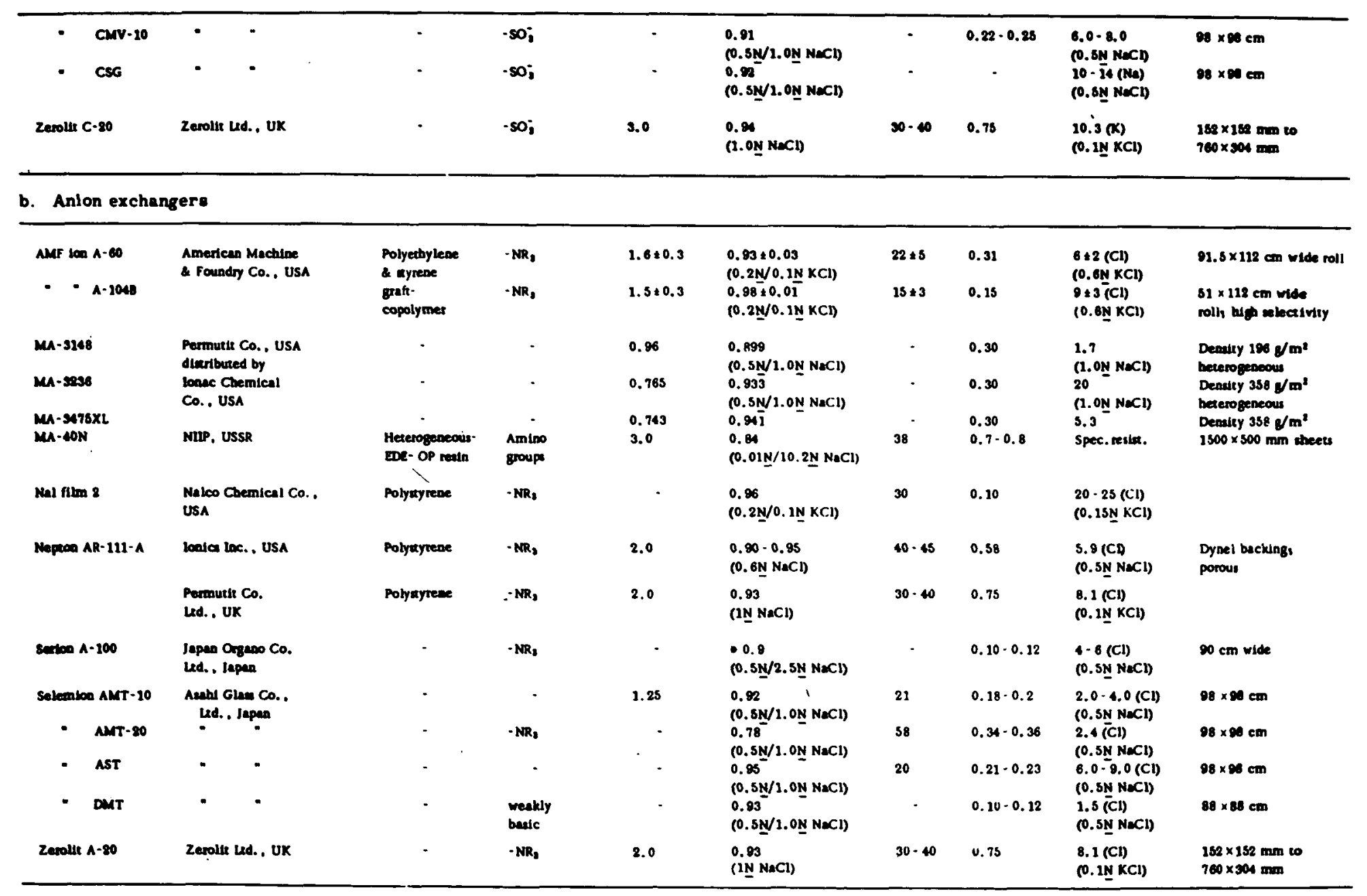

